\title{
Advances in LCMS Analysis of Lipids and Xenobiotics via Improvements in TrEnDi Derivatization
}

\author{
by
}

Samuel W.J. Shields

A thesis submitted to the Faculty of Graduate and Postdoctoral Affairs in partial fulfillment of the requirements for the degree of

Doctor of Philosophy

in

Chemistry

Carleton University

Ottawa, Ontario

(C) 2019, Samuel W.J. Shields 


\section{Abstract}

Mass spectrometry (MS) has become a critical technology for the analysis of complex mixtures by scientists across many fields of research. Its ability to identify and quantify small amounts of biomolecules has allowed for a deeper understanding of biological systems previously thought possible. The interaction between proteins, lipids, metabolites and DNA, etc. heavily relies on the use of diagnostics tools like mass spectrometry to gain a superior understanding of how humans are affected by disease and environmental exposure to xenobiotics. This thesis is focused on chemical derivatization techniques to improve the MS analysis of lipids and the commonly used pesticide, glyphosate. Chemical derivatization, or alternatively the application of chemical reactions to biologically relevant molecules is used to amplify the precision and accuracy of MS analyses.

The primary Chemical derivatization technique outlined here is Trimethylation Enhancement using Diazomethane (TrEnDi) whereby analytes undergo methylation to uniformly produce net positively charged species that contain amine functional groups. Glycerophospholipids, are a highly diverse class of biomolecules that can be readily methylated to net positively charged cations to greatly improve ionization efficiency prior to MS analysis. We successfully applied ${ }^{13} \mathrm{C}-\mathrm{TrEnDi}$ with improved conditions to methylate plasmalogen lipid species with no acidic cleavage of vinyl ether moiety. The identification of twenty-nine ${ }^{13} \mathrm{CH}_{3}$ plasmalogen lipids were observed from a complex lipid extract and shows increased signal-to-noise ratios compared to their unmethylated counterparts. Furthermore, a novel apparatus for delivering a small amount of diazomethane (DZM) to lipid samples was developed to decrease the handling of the 
hazardous reagent. Micro-scale delivery of diazomethane (iTrEnDi) was determined to be less effective than in solution derivatization of lipid samples but had greatly reduced the safety concerns associated with large-scale DZM preparations.

The most commonly used herbicide glyphosate and its major degradation product aminomethylphosphonic acid have been scrutinized for their long-term health effects and environmental impacts. Both compounds are challenging to analyze via classical LCMS methodologies due to low molecular weight, high polarity and anionic nature. Herein, iTrEnDi modification was used to produce permethylated GLY and AMPA with improved LC and ionization characteristics. 


\section{Preface}

This preface provides full bibliographical for articles and figures that are reproduced in this thesis. The use of copyrighted material is acknowledged here.

Pursuant to the Integrated Thesis policy of Carleton University, the "supervisors" (Jeff Manthorpe and Jeff Smith) as well as the student (Samuel Shields) confirm that the student was fully involved in the preparation of experiments, acquiring data, analyzing results, as well as preparing and writing these manuscripts including the co-authored articles in this thesis. Furthermore, the manuscripts prepared here that are not published and peer reviewed will be submitted to the appropriate journal prior to or shortly after the submission of a final thesis copy.

\section{Chapter 1}

Permission from Springer for "Chapter 11: Enhancing Analysis of Complex Lipid Samples Through Developments in Chromatography and Chemical Derivatization" In Molecular Technologies for Detection of Chemical and Biological agents. Banoub, J.; Caprioli, R.; NATO Science Peace Security Ser.A; Springer: Ney York, NY, 2017; 177-206 was granted for full or partial reproduction.

\section{Chapter 2}

Permission from ACS Publication for "Trimethylation Enhancement using ${ }^{13} \mathrm{C}$ Diazomethane $\quad\left({ }^{13} \mathrm{C}-\mathrm{TrEnDi}\right)$ : Increased Sensitivity and Selectivity of Phosphatidylethanolamine, Phosphatidylcholine and Phosphatidylserine Lipids Derived from Complex Biological Samples" Analytical Chemistry 2016, 6996-7004 was granted for full reproduction. 


\section{Chapter 3-5}

These chapters are written as manuscripts to be submitted to the Journal of the American Society for Mass Spectrometry. I have performed all experiments, MS analyses data interpretation and writing of documents. Other authors are acknowledged for their contributions to some proof of principal experiments suggested by me, proofreading documents and technical help.

\section{Chapter 7}

Section 7.1.2: Permission from ACS publication for "Trimethylation Enhancement Using ${ }^{13}$ C-DZM: Gas-Phase Charge Inversion of Modified Phospholipid Cations for Enhanced Structural Characterization" Analytical Chemistry 2017, 9452-9458 was granted to reproduce document for full or partial reproduction.

Section 7.1.3 and 7.2.2: These sections are the result of ongoing collaborations with Scott McLuckey's lab at Perdue University (IN, USA) and Jennifer Brodbelt's lab at University of Texas at Austin (TX, USA). I have reproduced figures containing data produced at the respective locations with permission. My role in these collaborations has been the synthesis of all modified lipid standards, interpretation of data, suggestion of the next steps to be performed and the editing of manuscript sections. 


\section{Acknowledgements}

To Jeff Manthorpe, (aka Jeff \#1) I have learned so much from you over these past seven or so years. You are a wealth of knowledge in science and beyond. I truly appreciate you giving me the opportunity to work in your lab as an undergrad student (who may have smelled kind of funny a lot of the time) and continued your mentorship through M.Sc. and now Ph.D. degrees. I can't thank you enough for the many hours of guidance you have given me on how to become an increasingly sophisticated scientist. You could say after I defend we will become chemical brothers (sorry had to toss in a bad pun since I have heard so many from you over the years). Also, in a way I have you to thank for bringing Diane and I together in your fourth-year organic chemistry class.

To Jeff Smith (aka Jeff \#2 or El Jefe), for real, when I started my Ph.D. I was unsure whether I would enjoy the next 5 years thinking about mass spectrometry. Well thanks to your guidance and mentorship I loved every minute of it. You have shown me that mass spectrometry is not just for dusty old dudes, but for people who enjoy hands on experience with instruments and the excitement of using mass spectrometry to understand the world around us. I cannot thank you enough for mentoring me in a field that will no doubt shape the rest of my career. Note: the Jeff numbering system is by chronological order in case you feel bad for being $\# 2$.

I would like to dedicate this thesis to my father Daniel Shields, who always encouraged me to follow a path of learning and understanding. Both my mother and father have inspired and supported me on the academic path I am now. From reading ingredients on cereal boxes at the dinner table to taking apart old computers, I truly thank the both of you for the support and love you have shown me. 
I want to say a big thanks to both Carlos and Karl for all the knowledge and training you have given me over the years. Both of you have allowed me to grow into a great chemist who can approach problem with a keen analytical chemistry eye that will benefit me for years to come. To the rest of the Smith/Manthorpe lab people, thank you for your help with this work and your fresh ideas whenever I was stuck on a problem.

Diane, my new wife, I can't thank you enough for the support over the years we have been together. Without you I am sure I would still be in an apartment on Gladstone eating only Pizza Shark three times a day. Thanks for your patience and I can't wait to travel the world with you.

Lastly, I would like to thank Chantelle and Caitlyn of the chemistry department administration for all the help with keeping me on track with my studies over the past 10 years. 


\section{Table of Contents}

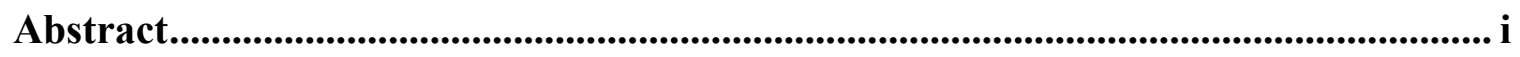

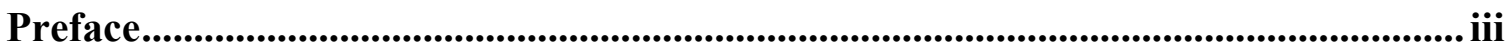

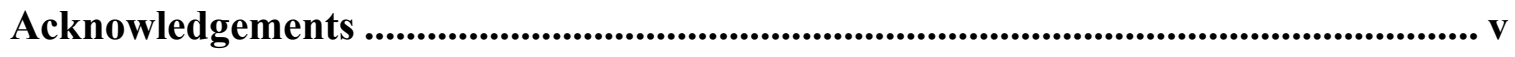

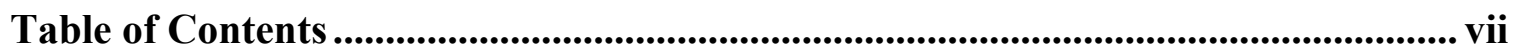

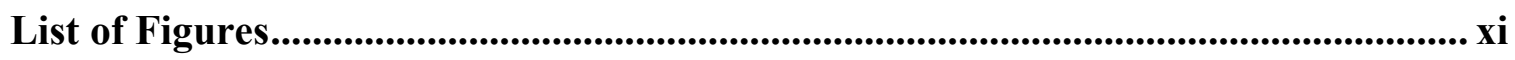

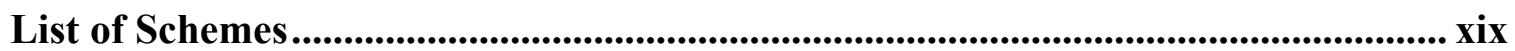

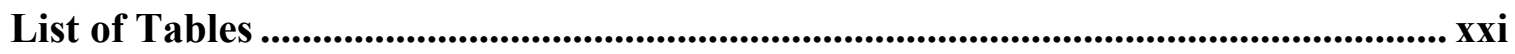

List of Abbreviations ................................................................................................ xxii

Chapter 1: Introduction ...................................................................................................... 1

1.1 Enhancing the Analysis of Complex Lipid Samples Through Developments in

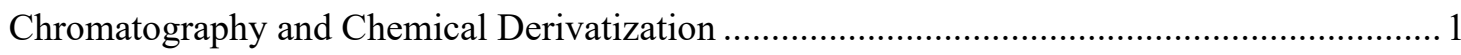

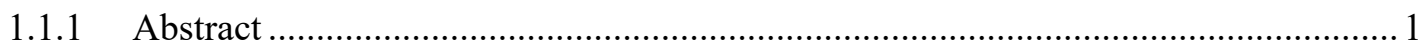

1.1.2 Lipidomics, Lipid Structure and Function ....................................................

1.1.3 Lipid Extraction, Separation and Analysis.......................................................

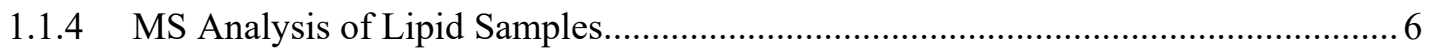

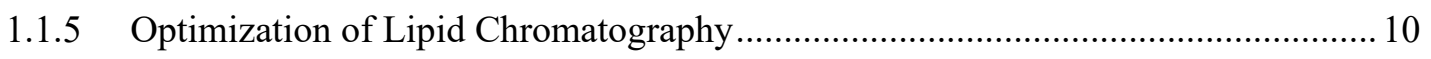

1.1.6 Chemical Derivatization to Improve Sensitivity in Lipidomics Analyses ............... 15

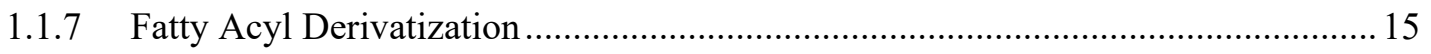

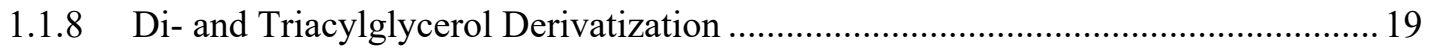

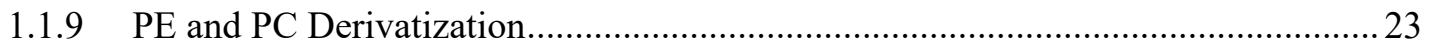

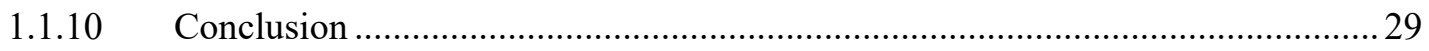

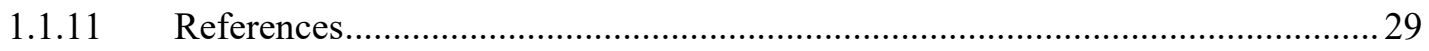

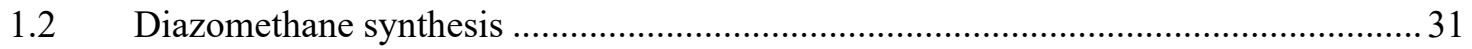


Chapter 2: Trimethylation Enhancement using ${ }^{13} \mathrm{C}$-Diazomethane $\left({ }^{13} \mathrm{C}\right.$-TrEnDi):

Increased Sensitivity and Selectivity of Phosphatidylethanolamine, Phosphatidylcholine and Phosphatidylserine Lipids Derived from Complex

Biological Samples ........................................................................................................ 34

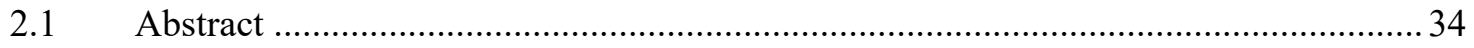

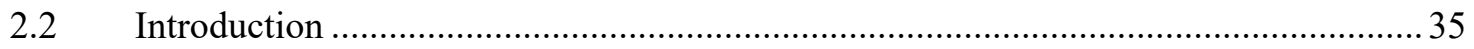

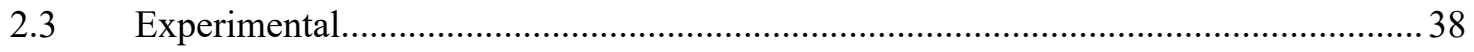

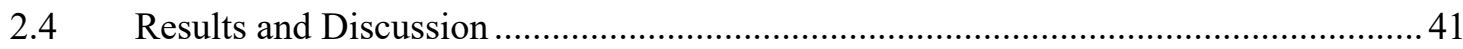

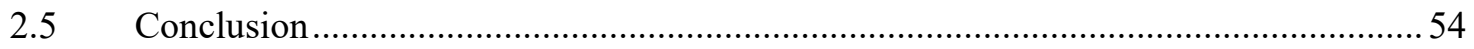

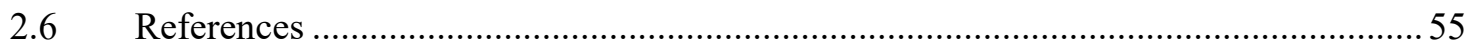

Chapter 3: Detection of Plasmenyl-Ether Glycerophospholipids in Bovine Liver Extract via a Dual Chemical Derivatization Strategy ............................................. 59

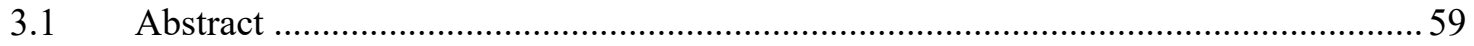

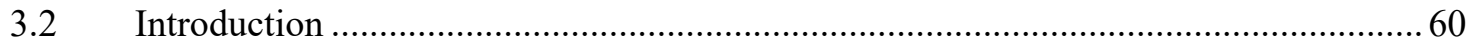

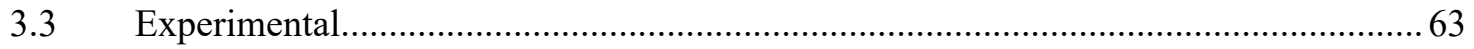

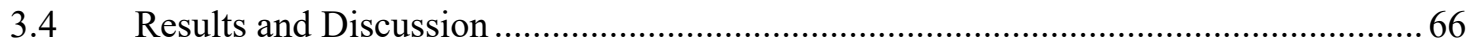

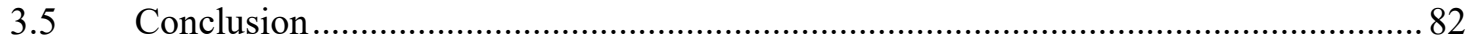

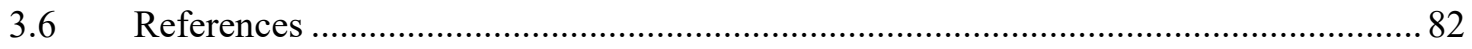

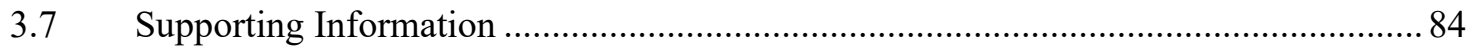

Chapter 4: Improved Analysis of Glycerphospholipids via a Micro-scale In Situ Derivatization Strategy using Diazoalkanes....................................................................... 88

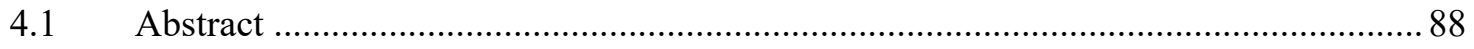

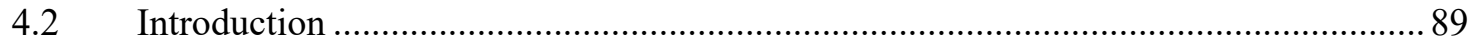

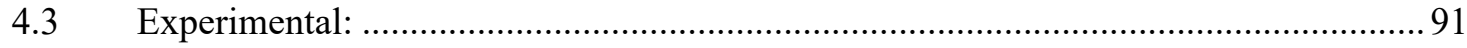

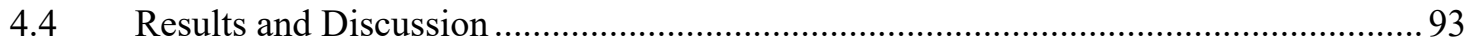

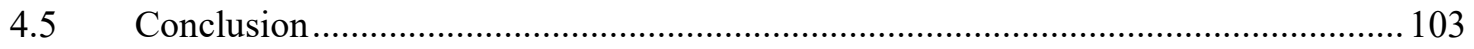

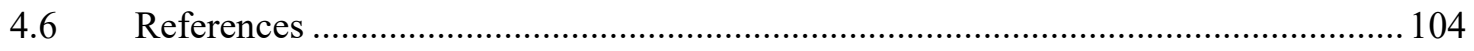




\section{Chapter 5: Determination of Glyphosate and Aminomethylphosphonic Acid Using}

A Novel Chemical Derivatization Technique and Analysis via LCMS................... 116

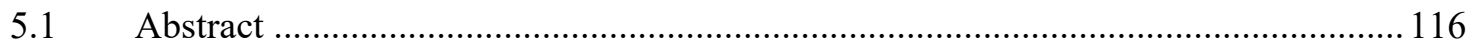

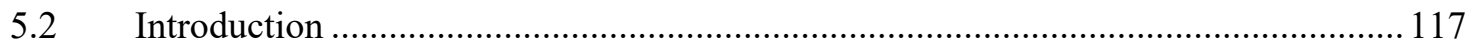

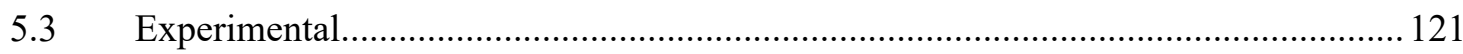

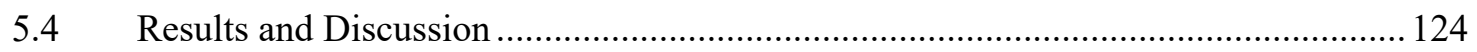

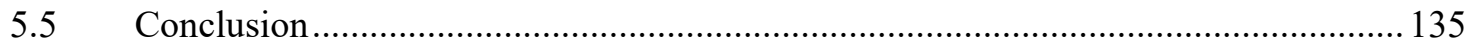

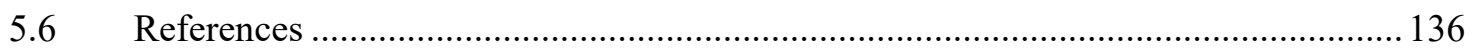

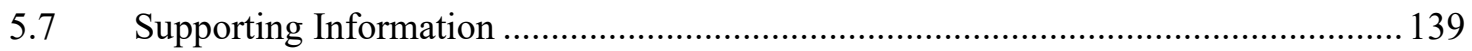

\section{Chapter 6: Solution-Phase Charge Inversion of Glycerophospholipids via complex}

formation with a spectrum of Diacid Structures .................................................... 143

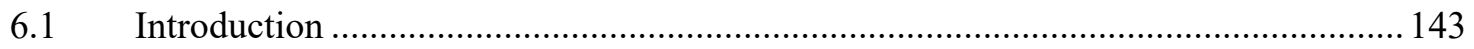

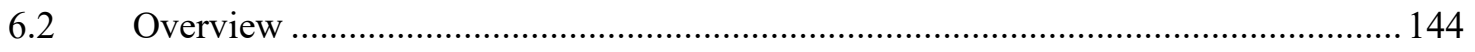

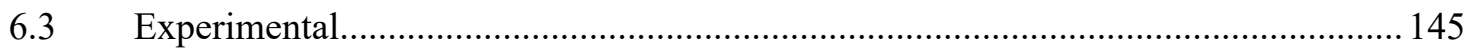

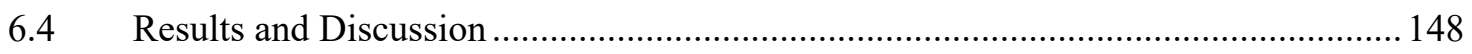

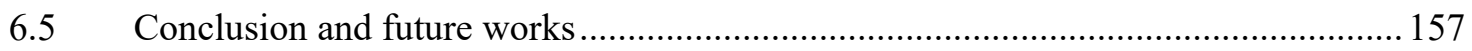

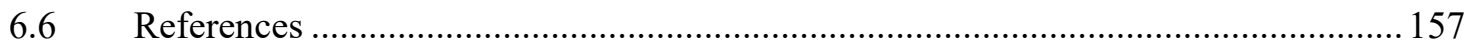

\section{Chapter 7: Additional Contributions to Studies of Derivatized}

Glycerophospholipids with Diazoalkanes ............................................................... 159

7.1 Gas-phase Charge inversion of ${ }^{13} \mathrm{C}$-TrEnDi Modified Glycerphospholipids .............. 159

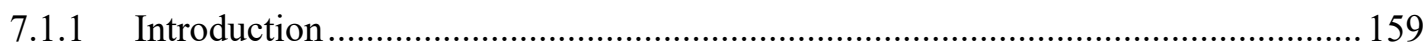

7.1.2 Trimethylation Enhancement Using ${ }^{13} \mathrm{C}-\mathrm{DZM}$ : Gas-Phase Charge Inversion of Modified Phospholipid Cations for Enhanced Structural Characterization ........................ 160

7.1.3 Determination of Double bond location in ${ }^{13} \mathrm{C}-\mathrm{TrEnDi}$ modified lipids using PDPA

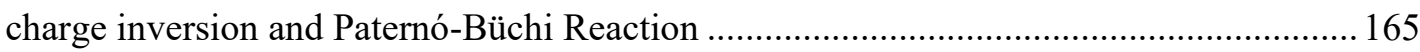


7.2 Ultraviolet photodissociation of ${ }^{13} \mathrm{C}$-TrEnDi modified glycerophospholipids ........... 169

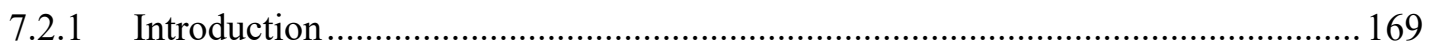

7.2.2 UVPD of TrEnDi Modified Glycerophospholipids ........................................... 175

7.3 Ethylation of PE(16:0/18:1) using Diazoethane produced in situ from ethylated nitroso urea 178

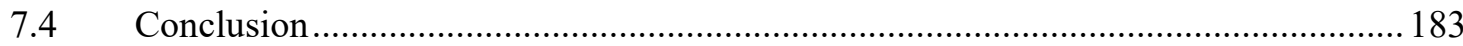

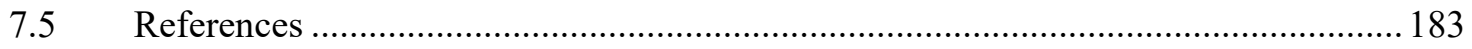

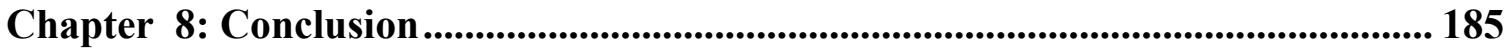

Appendices....................................................................................................................... 186

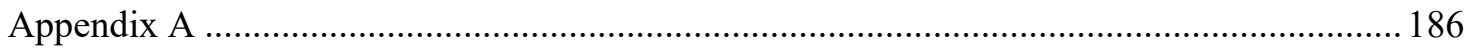

Bibliography or References.................................................................................................... 187 


\section{List of Figures}

Figure 1.1.1 Structural Features of Common Lipid Classes ............................................. 4

Figure 1.1.2 A General Workflow for Lipid Analysis ${ }^{13}$............................................ 10

Figure 1.1.3 Observation of lipid carryover within an HPLC system that has no column attached. (a) the ion current over the 60 min from the initial injection of lipid extract, (b) the average precursor ion mass spectrum over the course of the entire run, (c) the ion current over a 60 -min gradient after subsequently injecting $20 \mu \mathrm{L}$ of water through the system, (d) the average precursor ion mass spectrum over the course of the entire blank

run

Figure 1.1.4 Gas Chromatograph of FAMEs Derived from Eggs (left) and EI-MS of

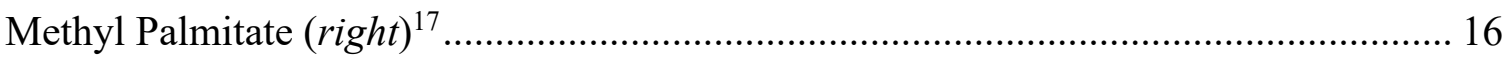

Figure 1.1.5 Quantitation of a Variety of 4-hydroxyalkenals Using Neutral Loss Scanning

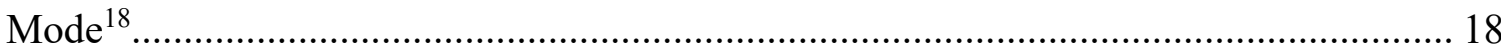

Figure 1.1.6 Optimization of Collision Energy for OzID (left) Possible Products of OzID

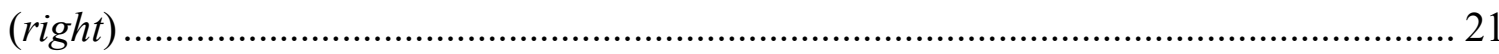

Figure 1.1.7 Neutral Loss of 103 Da of an Equimolar Mixture of DAG Standards ${ }^{22} \ldots . . .23$

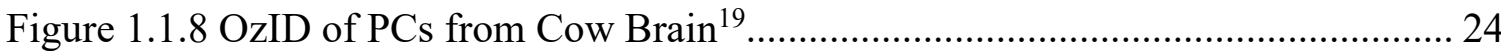

Figure 1.1.9 Unmodified lipid extract (top) and $d_{6}$-DMB modified lipid extract (bottom $)^{23}$

Figure 1.2.1 Two Methods of Preparing Ethereal Diazomethane From Diazald ${ }^{\mathrm{TM}}$ (A) and

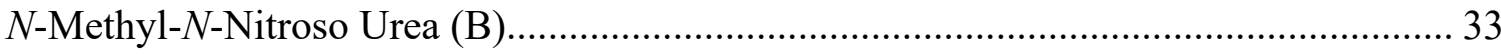

Figure 2.4.1 ${ }^{13} \mathrm{C}-\mathrm{TrEnDi}$ modification differentiates modified PE and PC species by unique PIS fragments of $\mathrm{m} / \mathrm{z} 199.1$ (A) and $\mathrm{m} / \mathrm{z} 202.1$ (B). 
Figure 2.4.2 Mass Spectrum of unmodified PE from HeLa cell lipid extract acquired via +NL scan of 141.1 Da (A). Mass spectrum of ${ }^{13} \mathrm{C}$-TrEnDi-modified PE from HeLa extract acquired via +PIS of m/z 202.1 (B).

Figure 2.4.3 Comparison of standard peak area averages between unmodified triplicates (red bars) and ${ }^{13} \mathrm{C}$-TrEnDi chemical triplicates (green bars) for (A) PE, (B) PC, and (C) SM extracted from HeLa cells 46

Figure 2.4.4 Unmodified HeLa cell lipodome was dissolved in EtOH with $10 \mathrm{mM}$ ammonium acetate or $10 \mathrm{mM}$ ammonium hydroxide and analyzed via their respective $+\mathrm{NL}$ of $185 \mathrm{Da}(\mathrm{A})$ or $-\mathrm{NL}$ of 87 Da scandal (B). The ${ }^{13} \mathrm{C}-\mathrm{TrEnDi}$-modified extracts were analyzed via +PIS of m/z 148.1 (C) and +PIS of m/z 261.1 (D). Unmodified 4x concentrated PS was analyzed using +NL 185.1 Da (E) 52

Figure 3.4.1 LCMS analysis of (a) un-treated lipid standards PE(16:0/18:1), PE(P18:0/18:1), PC(18:1/14:0), PC(P-18:1/14:0), (b) treated with 80\% formic acid in methanol, (c) treated with unoptimized TrEnDi conditions. Chromatograms are stacked with a Y-offset to clearly show each peak 67

Figure 3.4.2 Lipid standards treated with ${ }^{13} \mathrm{C}-\mathrm{DZM}$ and $10 \mathrm{mM} \mathrm{HBF}_{4}$ etherate. 70 Figure 3.4.3 Overall signal increase in the TIC of TrEnDi methylated BLE ( $\left.{ }^{\mathrm{Tr}} \mathrm{BLE}\right)$ over native lipids in the LCMS analysis of bovine liver extract. ${ }^{\text {Tr} B L E ~ i s ~ s t a c k e d ~ b y ~ Y ~ o f f s e t ~ o f ~}$

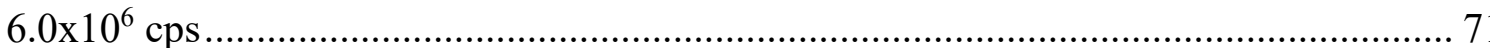

Figure 3.4.4 LCMS analysis showing extracted ion chromatograms for (a) an unmodified lipid species in untreated BLE with $\mathrm{m} / \mathrm{z}$ 752, (b) lipid species from the TrEnDi modified BLE with $\mathrm{m} / \mathrm{z} 767$, and (c) lipid species from the TrEnDi modified BLE with $\mathrm{m} / \mathrm{z} 812.73$ 
Figure 3.4.5 Plasmanyl ether lipids detected in unmodified BLE (black) and methylated lipids from the TrEnDi derivatized BLE (red), where error bars showing relative standard error of the average of three sequential injections (BLE) of chemical triplicates ( $\left.{ }^{\mathrm{Tr}} \mathrm{BLE}\right)$.

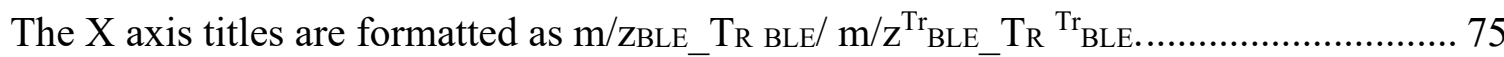

Figure 3.4.6 Kendrick Mass plot with $\mathrm{CH}_{2}$ Base of Unmodified Plasmenyl Ether-linked GPLs Identified by the Mild Acid Cleavage of BLE 76

Figure 3.4.7 Kendrick Mass Plot with $\mathrm{CH}_{2}$ Base of Methylated Plasmenyl Ether-linked GPLs Identified by the Mild Acid Cleavage of ${ }^{13} \mathrm{C}-\mathrm{TrEnDi}-\mathrm{BLE}$

Figure 3.4.8 Plasmalogen Lipids Identified from BLE showing the comparison of the extracted ion chromatograms of TrEnDi derivatized lipids (black) and corresponding $\operatorname{TrEnDi}+\mathrm{I}_{2}$ treated lipids $($ red $)$

Figure 3.7.1 (S1) Unmodified lipid standards left after the reaction with DZM using nonoptimized conditions 86

Figure 3.7.2 (S2) Acid concentration optimization on HeLa extract showing how $\left[\mathrm{H}^{+}\right]$ changes the abundance of fully modified plasmalogens. 87 Figure 3.7.3 (S3) Unmodified lipid standards left after the reaction with DZM using optimized conditions

Figure 4.4.1 Diagram of the experimental setup of iTrEnDi derivatization method. A) $\mathrm{N}_{2}$ gas source. B) diethyl ether- $\mathrm{N}_{2}$ formation vial. C) Diazoalkane reaction chamber $\left(\mathrm{H}_{2} \mathrm{O}\right.$, NMU, diethyl ether) D) Analyte vial (glycerophospholipids/sphingolipids) E) Base solution $\left(6.1 \mathrm{M} \mathrm{KOH}\right.$ in $\left.\mathrm{H}_{2} \mathrm{O}\right)$ 95 
Figure 4.4.2 LCMS and $\mathrm{MS}^{2}$ of derivatized lipid standards via iTrEnDi in situ methylation with DZM. a) PE 16:0/18:1. b) PC 19:0/19:0. c) SM d18:1/16:0. d) Over

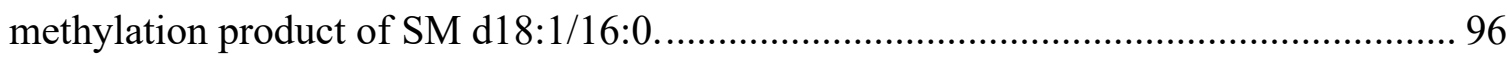
Figure 4.4.3 a) XIC of $\mathrm{m} / \mathrm{z} 554.38$ for underivatized PA 12:0/12:0 ammonium adduct. b) XIC of 582.41 for $\mathrm{PA}^{\mathrm{Tr}}$ 12:0/12:0 ammonium adduct. c) MS showing iTrEnDi-modified PA 12:0/12:0 with both ammoniated and sodiated adducts. d) $\mathrm{MS}^{2}$ of the ammoniated

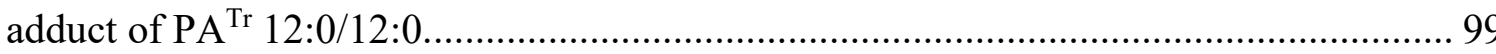

Figure 4.4.4 Comparison of in-solution TrEnDi vs. iTrEnDi using chemical triplicates to unmodified lipid standards 100

Figure 4.4.5 a) Equimolar mixture of unmodified, derivatized with DZM and derivatized with diazoethane PC 16:0/18:1, PC. b) $\mathrm{MS}^{2}$ analysis of diazoethane derivatized PC 16:0/18:1. c) Equimolar mixture of unmodified, derivatized with DZM and derivatized with diazoethane SM d18:1/16:0. d) $\mathrm{MS}^{2}$ analysis of dimethylated, [SM+2Me $]^{+}$, and monoethylated, $[\mathrm{SM}+\mathrm{Et}]^{+}, \mathrm{SM}$ d18:1/16:0. 102

Figure 4.7.1 (S1) Comparison of residue left after TrEnDi (1 mL, diazomethane) modification using DZM produced from a) distilled method with Diazald ${ }^{\mathrm{TM}}$ and $\mathrm{b}$ ) decanted method with NMU 106 Figure 4.7.2 (S2):XICs showing undermethylated species after the iTrendi reaction of PE 16:0/18:1 107 Figure 4.7.3 (S3) Chromatograms and $\mathrm{MS}^{2}$ data shown for ethylated phosphatidylcholine (PC 19:0/19:0). Unmodified PC resulted in an elution time of $11.23 \mathrm{~min}$, whereas ethylated PC eluted at $11.44 \mathrm{~min} . \mathrm{MS}^{2}$ data provided a dominant fragment at $212 \mathrm{~m} / \mathrm{z}$ represented by the modified headgroup of Et-PC. 108 
Figure 5.4.1 Signal splitting of glyphosate and AMPA between protonated and sodiated adducts in +MS (A). LCMS analysis of glyphosate and AMPA in +MS (B) and -MS (C) using multiple reaction monitoring 125

Figure 5.4.2 LCMS analysis of Me4-glyphosate using multiple reaction monitoring (A). Direct $+\mathrm{MS}$ of the reaction mixture produced from the iTrEnDi derivatized glyphosate (B). The $\mathrm{MS}^{2}$ of Me4- (C) and Me5- (D) GLY using LCMS and direct n-ESI-MS, respectively. 128

Figure 5.4.3 Full modification of glyphosate and AMPA with DZM using improved reaction conditions (A). Direct spray $\mathrm{nESI} \mathrm{MS}^{2}$ analysis of Mes-AMPA (B) and LC gradient optimization for peak shape of Mes-AMPA (C).

Figure 5.4.4 LCMS analysis of Glyphosate and AMPA with optimized multiple reaction monitoring parameters (left). Comparison of normalized peak area between TrEnDi modified $(5 \mu \mathrm{M})$ and unmodified $(25 \mu \mathrm{M})$ GLY and AMPA (right) 133 Figure 5.4.5 Calibration curve prepared for iTrEnDi derivatized GLY and AMPA (A) LCMS analysis of iTrEnDi modified herbicide from commercial Roundup ${ }^{\circledR}$ and soybean extract with optimized multiple reaction monitoring parameters (B). Values for $\mathrm{Me}_{5}-\mathrm{GLY}$ and -AMPA are multiplied by indicated factor to help visualization 135 Figure 5.7.1 (S1) Diagram of the experimental setup of iTrEnDi derivatization method. A) $\mathrm{N}_{2}$ gas source. B) diethyl ether- $\mathrm{N}_{2}$ formation vial. C) Diazoalkane reaction chamber $\left(\mathrm{H}_{2} \mathrm{O}\right.$, NMU, diethyl ether) or (diethylene glyocol monoethyl ether, $N$-methyl- $N$-nitrosop-toluenesulfonamide, diethyl ether) D) Analyte vial (glycerophospholipids/sphingolipids/small molecules) E) Base solution (6.1 M KOH in $\left.\mathrm{H}_{2} \mathrm{O}\right)$ 139 
Figure 5.7.2 (S2) nESI in +MS of iTrEnDi modified GLY and AMPA using optimized reaction conditions showing no unmethylated or undermethylated reaction products... 140 Figure 5.7.3 (S3) Sensitivity of unmodified AMPA (top) and GLY (bottom) .............. 140 Figure 5.7.4 (S4) Sensitivity of derivatized AMPA (top) and GLY (bottom)............... 141 Figure 6.2.1 Four diacids to be investigated via solution phase charge inversion of TrEnDi modified PC 144

Figure 6.4.1 nESI-MS positive spectrum of $\mu \mathrm{M}$ PC with $10 \mathrm{mM} \mathrm{NH} 4 \mathrm{OAc}$ in ethanol showing protonated and sodiated adducts........................................................ 148

Figure 6.4.2 nESI-MS ${ }^{2}$ positive product ion spectrum of $\mathrm{m} / \mathrm{z} 732.6 \ldots \ldots \ldots \ldots \ldots \ldots \ldots \ldots \ldots . . . . . . . . . . . .149$

Figure 6.4.3 nESI-MS positive spectrum of TrEnDi modified $50 \mathrm{mM}$ PC in EtOH..... 150

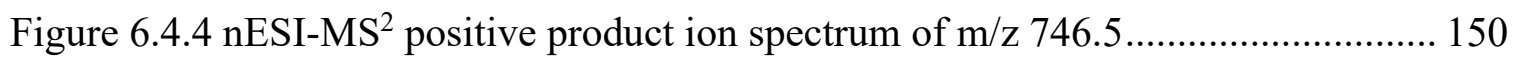

Figure 6.4.5 nESI-MS negative spectrum of TrEnDi derivatized PC with TDPPA...... 153

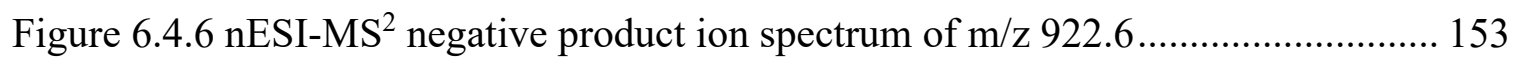

Figure 6.4.7 nESI-MS negative spectrum of $\mathrm{NaBPh}_{4} 1 \mu \mathrm{M}$ in $\mathrm{EtOH}$.......................... 154

Figure $6.4 .8 \mathrm{nESI}-\mathrm{MS}^{2}$ negative product ion spectrum of underivatized $[\mathrm{PC}+\mathrm{TDPPA}]^{2-}$

Figure 7.1.1 Ion-ion reaction products of tmPE tmPC and tmPS standards with PDPA 162 Figure 7.1.2 Ion-ion reaction between reagent dianion [PDPA-2H] ${ }^{-}$and (a) tmPC, (b) tmPE and (c) tmPS. (•) Ions are present prior to cation injection. 163 Figure 7.1.3 Ion-trap CID of (a) [tmPC- $\left.\left(\mathrm{CH}_{3}\right)_{3} \mathrm{NCH}_{2} \mathrm{CH}_{2}\right]^{-}$, (b) [tmPE- $\left.\left(\mathrm{CH}_{3}\right)_{3} \mathrm{NCH}_{2} \mathrm{CH}_{2}\right]^{-}$, [tmPS- $\left.\left(\mathrm{CH}_{3}\right)_{3} \mathrm{NCH}_{2} \mathrm{CH}_{2}\right]^{-}$. Lightning bolt signifies activated ions. 164 Figure 7.1.4 Combination of PB reaction and charge inversion of tmPE. a) The PB reaction spectrum of $5 \mu \mathrm{M}$ tm PE 16:0/18:1(9Z) after 5 seconds of UV exposure, b) Gas- 
phase ion/ion reaction of $\left[{ }^{\mathrm{PB}} \mathrm{tmPE}\right]^{+}$and $\left.[\mathrm{PDPA}-2 \mathrm{H}]^{2-}, \mathrm{c}\right)$ Ion trap CID of $\left[{ }^{\mathrm{PB}} \mathrm{tmPE}+\right.$ PDPA $-2 \mathrm{H}]^{-}$followed by ion trap CID of $\left[{ }^{\mathrm{PB}} \mathrm{tmPA}\right]^{-}$, d) Ion trap CID of the PB product of $\mathrm{C} 18: 1(9 \mathrm{Z})$ verifying the $\mathrm{C}=\mathrm{C}$ position of $\mathrm{PC} 16: 0 / 18: 1\left(\Delta^{9}\right)$. CID of a target ion is depicted by a lightning bolt $(\mathbb{N})$ 167

Figure 7.1.5 a) Charge inversion of positive ion mode $\mathrm{m} / \mathrm{z} 860$ (the PB product of 802 ), and sequential CID spectrum of $m / z 339$ (a) and sequential CID spectrum of $m / z 337$ (b). CID of a target ion is depicted by a lightning bolt $(\mathbb{N})$ 169

Figure 7.2.1 Schematic of the Orbitrap Fusion Lumos tribrid mass spectrometer from Thermo Scientific ${ }^{15}$

Figure 7.2.2 Chemical structures of diphosphatidyltrehalose, cardiolipin (18:1/18:1/18:1/18:1) and mycolic acid (A). Diagnostic fragments produced via a hybrid CID-UVPD $\mathrm{MS}^{2}$ analysis of $\left.[\mathrm{diPT}+\mathrm{Na}]^{+},{ }^{2 \mathrm{CL}-2 \mathrm{H}}\right]^{-}$, and $[\mathrm{MA}-\mathrm{H}]^{-}$enabling a complete structure elucidation (B) 174

Figure 7.2.3 HCD (top) and UVPD (bottom) product ion spectra of [tmPE(16:0/18:1) $]^{+}$

Figure 7.2.4 Summed UVPD DB frag ion peak area vs. \#mol injected

Figure 7.2.5 UVPD fragmentation of $\mathrm{m} / \mathrm{z} 750.57$ (A) and 778.62 (B) from ${ }^{13} \mathrm{C}-\mathrm{TrEnDi}$ modified polar E.coli extract analyzed by LCMS 178 Figure 7.3.1 Structures of PE (16:0/18:1) and ethylated products produced via the reaction with diazoethane 180 Figure 7.3.2 LCMS analysis showing of PE (16:0/18:1) prior to reaction with diazoethane (A) and mono-, di-, tri-, and tetra ethylated products produced from the reaction (B-E), respectfully. 180 
Figure 7.3.3 Tandem MS analysis of mono- (A), di- (B), tri-(C) and tetra-ethylated (D) PE products produced in the reaction with diazoethane ........................................... 182

Figure 7.3.4 Identified fragments from the analysis of ethylated PE products via $\mathrm{MS}^{2} .182$ 


\section{List of Schemes}

Scheme 1.1.1 Biosynthesis of the most abundant lipids in humans ${ }^{7}$............................... 5

Scheme 1.1.2 Synthesis of Fatty Acid Methyl Esters..................................................... 16

Scheme 1.1.3 General Reaction Scheme and Mechanism of Adduct Formation of 4-

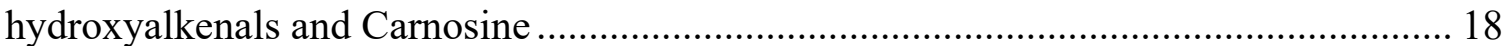

Scheme 1.1.4 General Reaction and Mechanism of the Ozonolysis of Triacylglycerols. 20

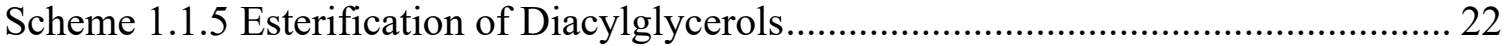

Scheme 1.1.6 Chemical Derivatization of PEs with $d_{6}$-DMBNHS General Reaction and

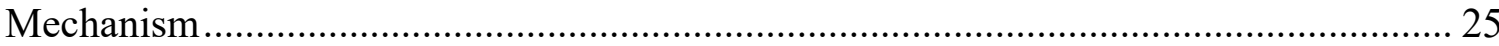

Scheme 1.1.7 Reaction of Diazomethane with PE General Reaction and Mechanism .... 28

Scheme 1.1.8 ${ }^{13} \mathrm{C}-\mathrm{TrEnDi}$ derivatization of $\mathrm{PC}(16: 0 / 18: 1)$ and $\mathrm{PE}(16: 0 / 18: 1)$.............. 29

Scheme 2.4.1 TrEnDi Derivatization of PC(16:0/18:1) and $\mathrm{PE}(16: 0 / 18: 1)$ with ${ }^{13} \mathrm{CH}_{2} \mathrm{~N}_{2}$

Scheme 3.4.1 Cleavage of plasmenyl ether lipids via formic acid and unoptimized

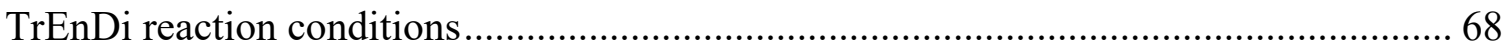

Scheme 3.4.2 Methylation of PC and PE plasmalogen standards with ${ }^{13} \mathrm{C}-\mathrm{DZM}$ and 10

$\mathrm{mM} \mathrm{HBF} 4$ produces methylated species with no cleavage of vinyl ether ........................ 71

Scheme 3.4.3 Two-step derivatization of plasmalogen lipids using ${ }^{13} \mathrm{C}-\mathrm{DZM}$ and $\mathrm{I}_{2}$ to produce a methylated GPL with fixed positive charge and iodoacetylated vinyl ether.... 80

Scheme 4.7.1 Preparation of $N$-methyl- $N$-nitroso urea............................................... 108

Scheme 4.7.2 Preparation of $N$-ethyl- $N$-nitroso urea..................................................... 109

Scheme 5.2.1 Proposed Degradation of Glyphosate in Soil by Microorganisms........... 118 
Scheme 5.2.2 Recent derivatization strategies for LCMS analysis of glyphosate and AMPA

Scheme 5.4.1 Reaction products produced by the reaction of DMZ and glyphosate (left).

Proposed fragmentation mechanisms for $\mathrm{MS}^{2}$ fragments observed for Me5-glyphosate

(right)

Scheme 6.1.1 The charge inversion of PC using PDPA in an ion trap

Scheme 6.3.1 Synthesis of RDAA

Scheme 7.1.1 Gas-phase charge inversion with PDPA and fragmentation of PC and PE

lipids 160

Scheme 7.1.2 Sequence of events for tmPL structure determination using off-line

Paternó-Büchi [2+2] cycloaddition and ion/ion reaction products analized via $\mathrm{MS}^{4} \ldots . .166$

Scheme 7.2.1 UVPD Ion Activation with 263 nm Laser to Cause Fragmentation ........ 170 


\section{List of Tables}

Table 1.1.1 Sensitivity Comparison of Methylated PE and PC Against Non-Modified

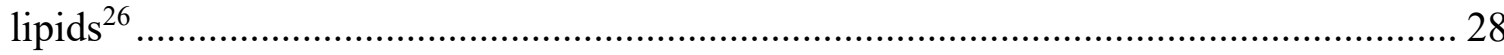

Table 2.4.1 Summary of ${ }^{13} \mathrm{C}$-TrEnDi Enhancements on Identification and Quantitation of

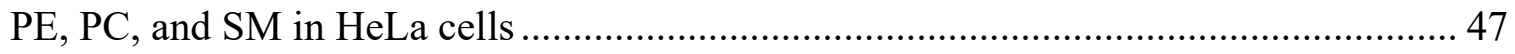

Table 3.4.1 Optimization of $\mathrm{HBF}_{4}$ etherate concentration for the complete methylation of

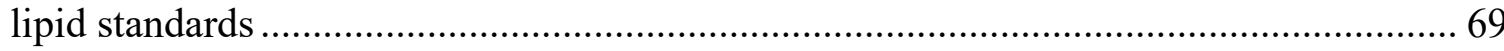

Table 3.4.2 Summary of the Comparison of TrEnDi Modified Plasmalogens to

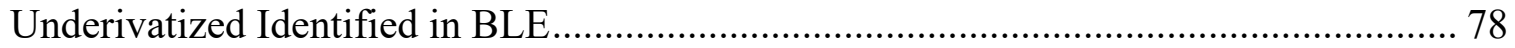

Table 3.7.1 (S1) Full list of UNMOD plasmalogens identified in BLE that have greater than $50 \%$ decrease in peak area after formic acid treatment ................................... 84

Table 3.7.2 (S2) List of TrEnDi modified plasmalogens in BLE .................................. 85

Table 4.7.1 (S1) Complete list of materials used to assemble the iTrEnDi experimental

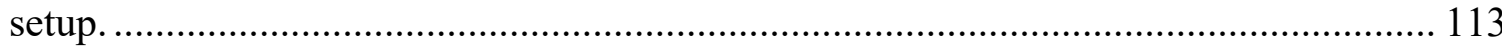

Table 5.3.1 MRM parameters used for LCMS analysis ......................................... 123

Table 5.7.1 (S1) Sensitivity enhancements of iTrEnDI modification of GLY and AMPA.

Table 6.4.1 Results of diacid-lipid adduct formation in negative ion mode.................. 152

Table 6.4.2 Peak areas of comparison experiment ............................................... 155

Table 6.4.3 Normalized peak areas of comparison experiment................................ 155 


\section{List of Abbreviations}

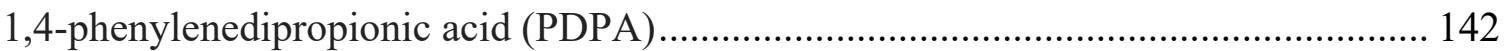

${ }^{13} \mathrm{C}-\mathrm{TrEnDi}$ modified phospholipid (tmPL) ............................................................... 160

6-aminoquinolyl- $N$-hydroxysuccinimidyl carbamate ${ }^{7}$ (AQC)..................................... 118

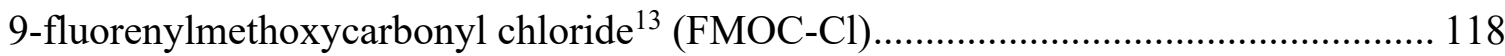

acetonitrile $(\mathrm{ACN})$

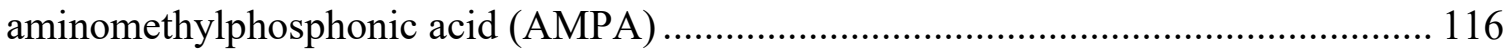

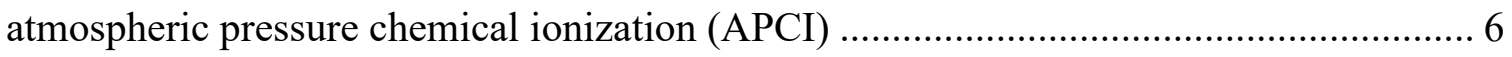

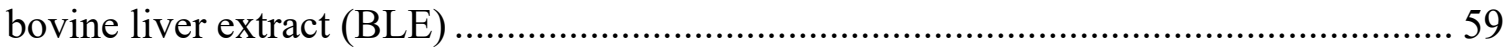

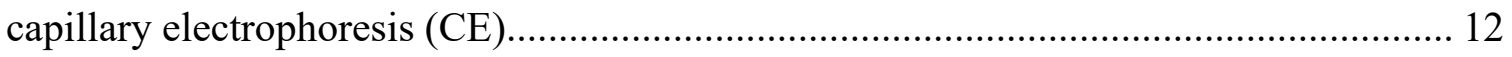

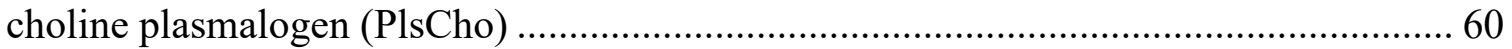

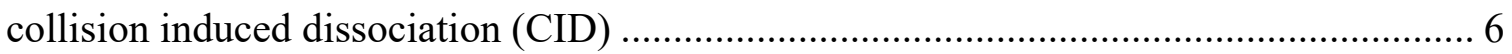

$d_{6}$-dimethylthibutanoylhydroxysuccinimide ester (d6--DMBNHS) ............................... 24

diacylglycerols (DAG)

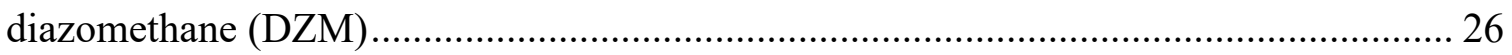

dimethylglycine (DMG)

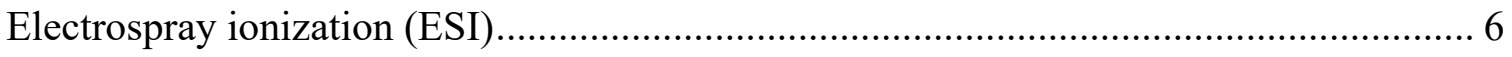

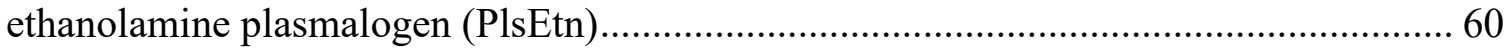

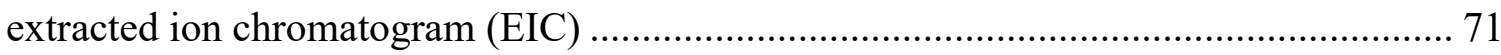

fatty acid methyl ester (FAME) ………….......................................................... 15

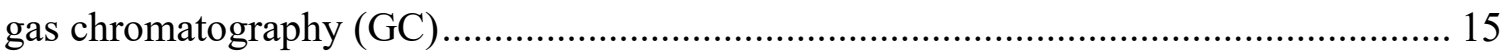

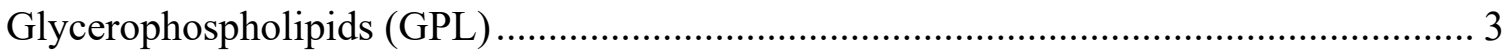


Glyphosate (GLY)

High performance liquid chromatography (HPLC or LC) .......................................... 8

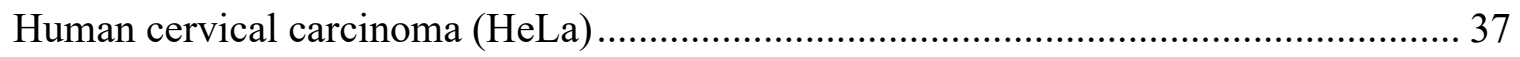

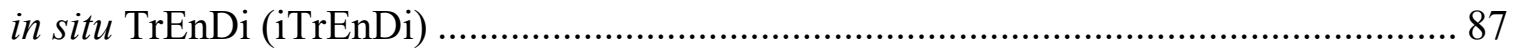

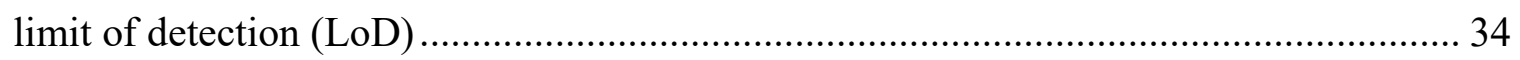

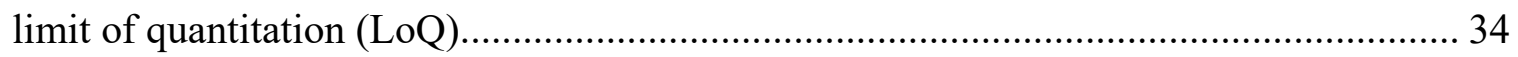

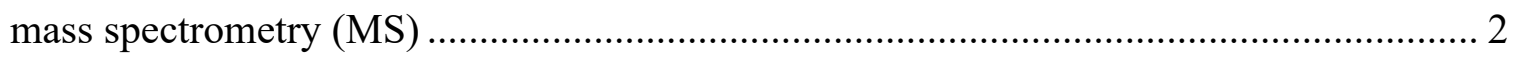

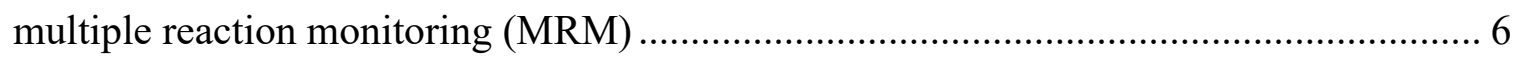

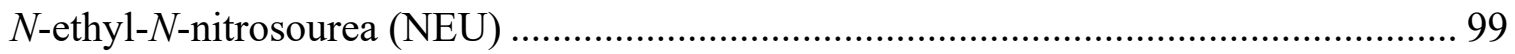

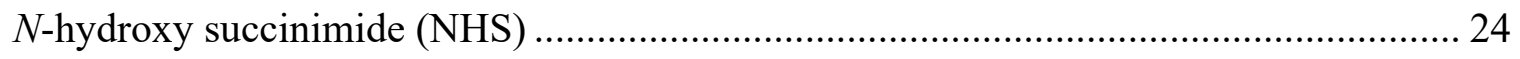

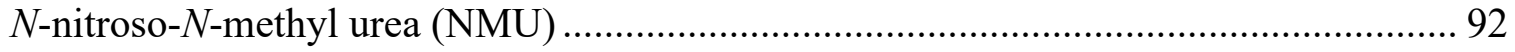

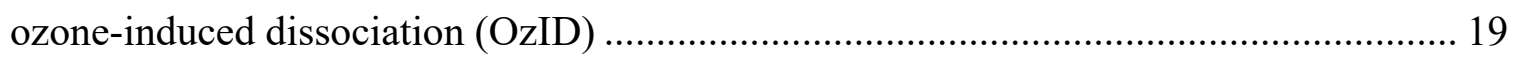

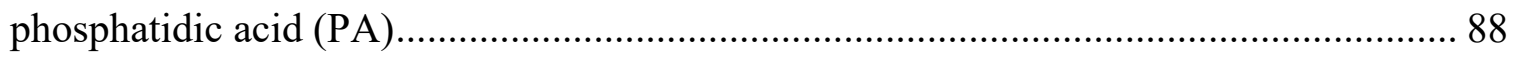

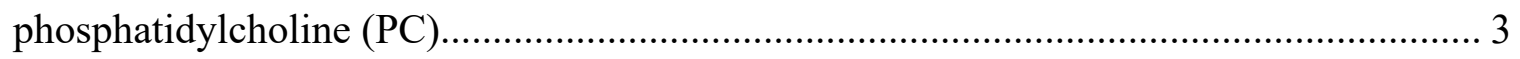

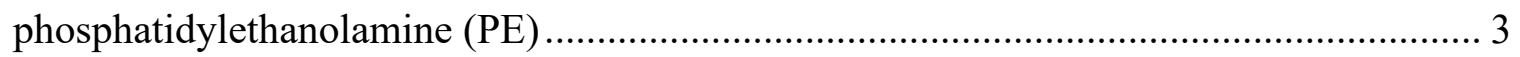

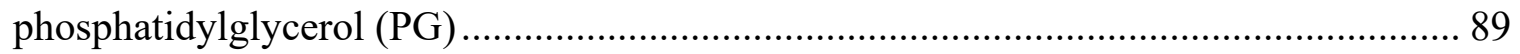

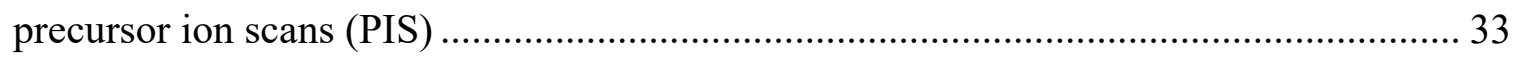

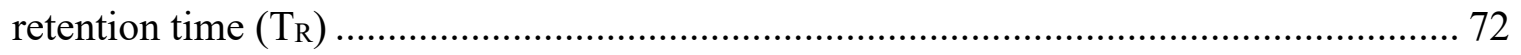

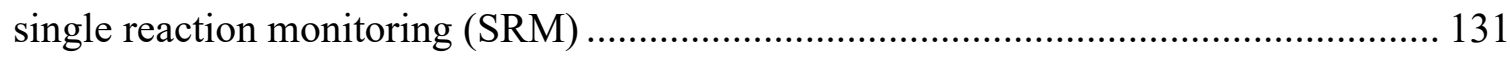

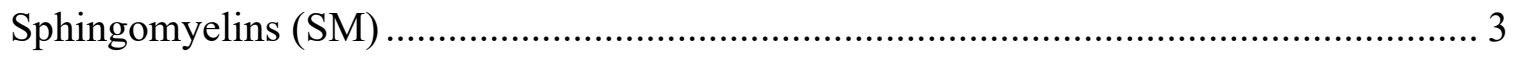

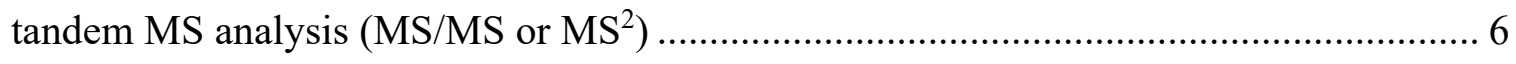

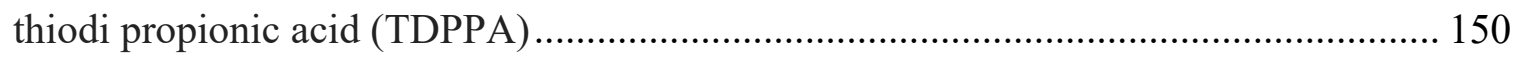

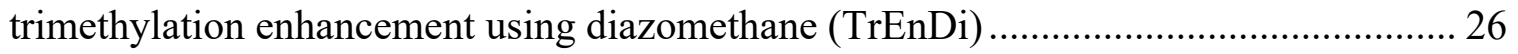




\section{Chapter 1: Introduction}

\subsection{Enhancing the Analysis of Complex Lipid Samples Through Developments in Chromatography and Chemical Derivatization}

Adapted with permission from Carlos R. Canez, Karl V. Wasslen, Hyunmin Lee, Danisz

Stalinski, Lennart Trouborst, Shira Joudan, Sarah Whitton, Hillary P. Weinert, Jeffrey M. Manthorpe, and Jeffrey C. Smith. Molecular Technologies for Detection of Chemical and Biological agents. Banoub, J.; Caprioli, R.; NATO Science Peace Security Ser.A; Springer: Ney York, NY, 2017; 177-206

\subsubsection{Abstract}

Lipids are one of the main building blocks of cellular life and represent the most abundant class of biomolecule by weight. Some of their most obvious roles include providing a source of energy storage within cells and creating barriers to compartmentalize cells by forming boundaries between cells and organelles. More recently, the roles that lipids and lipid metabolites play in cellular signaling have been increasingly revealed by the scientific community, fueling interest in this field. Chromatography coupled to mass spectrometry has emerged as an eminent analytical tool with which to study lipids, however not without challenges along the way. This chapter first summarizes some of the recent developments in the use of chromatography to separate complex lipid samples in an efficacious manner including the presentation of new techniques to increase precision and avoid sample carry-over. The latter part of the chapter details recent strategies to chemically derivatize lipids to enhance their analytical characteristics for separation and 
analysis by mass spectrometry with the aim to gain greater insight into the roles that these biomolecules play in living systems.

\subsubsection{Lipidomics, Lipid Structure and Function}

Lipidomics is an ever-expanding field that studies lipids and their interactions within biological systems. ${ }^{1}$ A large portion of the field is devoted to the structural characterization and quantitation of lipids to gain insight into their function. The goal of lipidomics research is to obtain the full characterization of lipid species and their specific biological roles within the context of all other cellular mechanisms (e.g. gene transcription, protein translation and other metabolomic processes). This goal can be realized by several different methods but few as sensitive and powerful as mass spectrometry (MS). MS has been used in lipidomics research to find and identify potential lipid biomarkers, compare two or more lipid profiles, discover new lipids and lipid metabolites and provide quantitative information on these analytes in the context of complex biological systems ${ }^{1}$.

Abnormal lipid behavior in humans has been implicated in a variety of pathologies including diabetes, cancer, and some neurodegenerative diseases. Apart from being energy storage molecules, lipids are involved in many biochemical functions related to disease states such as: signal transduction pathways, proliferation, apoptosis and membrane

trafficking in the cell. ${ }^{2-5}$ Accordingly, the field of lipidomics is of great interest to many factions of the health sciences.

Lipids are generally non-polar or amphiphilic molecules and usually possess large hydrocarbon (non-polar) chains and in some cases a polar head group (e.g. carboxyl, phosphate, amino, or carbohydrates). Figure 1.1.1 depicts some of the most common lipid 
classes. Although lipids are composed of very simple building blocks, they have the potential for $\sim 100,000$ unique molecular species. ${ }^{6}$ With each of the different lipid structures and classes having important biological roles to play, lipidomics has become one of the leading fields in health research with the potential for many significant clinical applications.

Each structural variant of lipids can have many different functions within a biological system. For example, fatty acyl lipids can be used in fatty acid oxidation for the generation of energy for cells but also are the main building block for other higher order biomolecules. ${ }^{7}$ In vivo, fatty acyl chains can be converted to mono-, di-, or triacylglycerols which are the main long-term energy storage biomolecules for mammals (Scheme 1.1.1). Along the same pathway glycerophospholipids can also be produced from the 1,2-diacyl glycerols. Glycerophospholipids (GPL) are comprised of a polar phosphate head group and two fatty acyl chains labeled as $s n-1$ and $s n-2$ (Fig. 1.1.1) and are subdivided into sub-classes depending on the phosphate head group and the nature of the fatty acyl chains. Phospholipids make up $\sim 60 \%$ of a cells lipidome with phosphatidylethanolamine (PE) and phosphatidylcholine (PC) classes composing $75 \mathrm{~mol} \%$ of eukaryotic membranes. ${ }^{8}$

Two other glycerophospholipids that play important roles in nervous system and cardiovascular system cellular membranes are sphingolipids and plasmalogens. Sphingomyelins (SM) are characterized by having a polar phosphate group, a fatty N-acyl group at the $s n-2$ position, and a fatty allylic alcohol moiety at $s n-1$. These lipids make up a large percentage of the myelin sheath of neurons that insulates the axon allowing for signal transduction. ${ }^{9}$ Abnormalities in sphingolipid composition of neuronal cells can lead 
to neurodegenerative disorders like multiple sclerosis. ${ }^{9}$ Plasmalogens are characterized by having a polar phosphate group, a vinylic fatty ether component at $s n-1$ position, and a fatty acyl substituent at the $s n-2$ position. Aberrant plasmalogen behavior can be linked to Alzheimer's disease. ${ }^{10}$ It is hypothesized that many other lipids play roles in disease states in the human body and therefore new technologies and analytical techniques are required to accurately determine lipid structure and ascertain the composition of complex lipidomics in order to progress towards a full understanding of the diseases that plague humankind.

Fatty Acyls
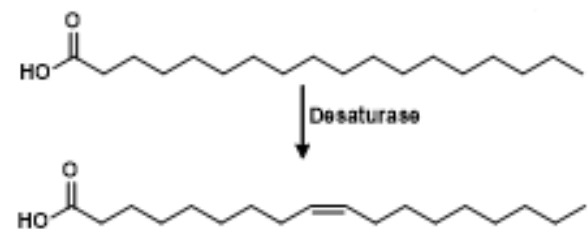

Acyiglycerols
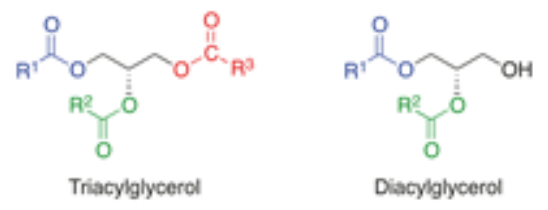

Glycerol backbone
sn-1, $5 n \cdot 2,5 n \cdot 3$

Tnacylglycercl

Discylglycerol

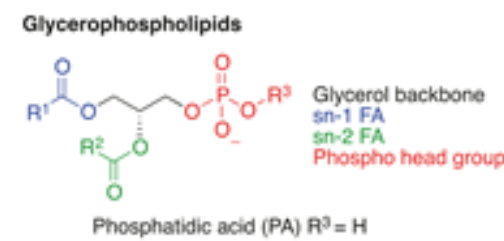

Phosphatidic acid $(\mathrm{PA}) \mathrm{R}^{3}=\mathrm{H}$

Phophatidylcholine (PC) $\mathrm{R}^{3}=$

Phosphatidylethanolamine (PE) $\mathrm{R}^{3}=\mathrm{N}_{\mathrm{O}}$
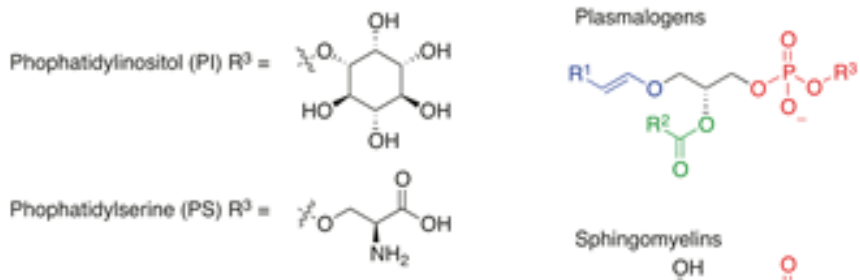

Phephatidylglycerol (PG) $\mathrm{R}^{3}=$

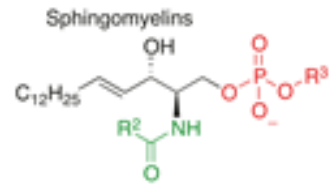

Figure 1.1.1 Structural Features of Common Lipid Classes 


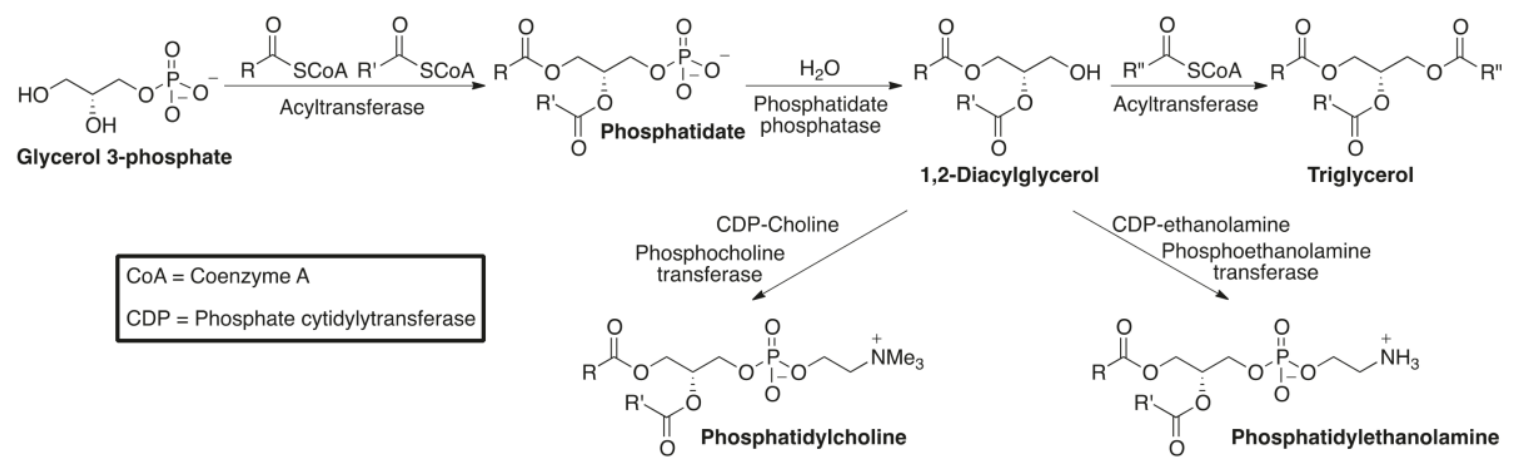

Scheme 1.1.1 Biosynthesis of the most abundant lipids in humans ${ }^{7}$

\subsubsection{Lipid Extraction, Separation and Analysis}

To analyze lipids and gain insight into cellular function, they must first be extracted from a biological sample. Biological samples contain many interfering compounds such as carbohydrates, nucleic acids, proteins, and salts that must be first separated from the lipids. The most common approach to extract lipids is to use some variation of a micro-scale liquid-liquid extraction. Briefly, the biological sample is homogenized and suspended in aqueous and alcoholic media with an organic solvent (e.g. chloroform, ethanol, benzene) to form a monophasic system. The mixture is then agitated vigorously and centrifuged at high speeds to obtain a biphasic system. The non-polar layer can then be removed, and the remaining aqueous layer re-extracted with more non-polar solvent. ${ }^{11,12}$ The most common solvent extraction technique that follows these steps is the Bligh and Dyer extraction and the resulting extract is often complex and contains a wide variety of different lipids. Variations on the Bligh and Dyer extraction include modifications designed to remove some lipid classes over others to obtain a more simplified sample, while others control $\mathrm{pH}$ using buffers or alter the ratio of alcohol to water to modulate the disruption of lipid-protein complexes. ${ }^{13}$ 


\subsubsection{MS Analysis of Lipid Samples}

MS is a powerful tool that may be used to analyze complex lipid samples containing hundreds to thousands of analytes. Electrospray ionization (ESI) and atmospheric pressure chemical ionization (APCI) have demonstrated their utility to ionize non-volatile lipid analytes and are most commonly cited in the lipidomics literature. ${ }^{13,14}$ Electrospray ionization is a technique uses the flow of solvent through a capillary that has a potential of 2-5000 kV. The electric field and solvent pressure distorts the solvent at the end of the capillary to form a Taylor cone which emits small charged droplets. Through evaporation and columbic repulsion larger droplets become smaller until they reach approximately 1$10 \mathrm{~nm}$ in size. ${ }^{14 \mathrm{~b}}$ At this point there is some discussion in the literature as to how analaytes become gas-phase $[\mathrm{M}+\mathrm{H}]^{+}$species that are dependent on the solvent composition and analyte structure. The ion evaporation model (IEM) ${ }^{14 \mathrm{c}}$ can be used to describe how small non-polar ion are ejected from a droplet. Computational studies have shown that in a droplet where ions are solvated with methanol and water $(1: 1)$ very polar species like $\mathrm{Na}^{+}$ reside closer to the center of the droplet whereas more non-polar species reside closer to the surface. ${ }^{14 \mathrm{c}}$ Due to columbic repulsion of similarly charged ions at the surface, they are ejected from the droplet to form gas-phase species. In the case of lipids ionization by IEM is most likely due to their non-polar nature and solubility in aqueous solvents.

In the case of glycerophospholipids, triple quadrupole or hybrid quadrupole instruments are used to analyze the composition of the lipidome by single MS scans and tandem MS analysis (MS/MS or $\mathrm{MS}^{2}$ ). $\mathrm{MS}^{2}$ analysis helps to provide information on the fatty acyl components (product ion scan in negative ion mode), the polar head group (product ion scan in positive ion mode) and analyze the composition of a particular lipid 
class (precursor and neutral loss scans). There are many challenges in analyzing lipids via MS such as their amphipathic nature, low proton affinity, vast and often subtle structural diversity, wide range of abundances and low relative abundance of some lipid biomarkers.

In the case of a triple quadrupole instrument, $\mathrm{MS}^{2}$ data is collected by selecting a small bandwidth ( 1 Da) of ions in the first quadrupole (Q1), colliding these ions in the second quadrupole (q2) with an inert gas to induce fragmentation (collision induced dissociation (CID)) and scanning the $\mathrm{m} / \mathrm{z}$ values of the resulting fragments in the third quadrupole (Q3). There are four different $\mathrm{MS}^{2}$ scanning modes that this instrument geometry affords that are most commonly employed in lipidomics analyses, product ion scanning, precursor ion scanning, neutral loss scanning and multiple reaction monitoring (MRM). Production ion scanning uses Q1 to select a single ion of interest and fragment it in q2 via CID where Q3 scans and reports the $m / z$ values of the resulting fragments. The mass spectrum of a product ion scan displays the ion fragments that originate from a single ion in the sample, which provides structural information about the molecule. Precursor ion scanning is the inverse of product ion scanning in that Q1 scans a range of $m / z$ ratios that in turn are fragmented in q2 whereas Q3 is set to select only one of the fragment ions. The final mass spectrum displays the parent ions that fragmented in Q2 to yield a specific $\mathrm{m} / \mathrm{z}$ fragment that passed through Q3. Neutral loss scanning employs both Q1 and Q3 scanning a range of $\mathrm{m} / \mathrm{z}$ values offset by a constant amount; ions that fragment in q2 and lose a specific neutral mass will successfully transmit through Q3. The final mass spectrum will display the $\mathrm{m} / \mathrm{z}$ of ions that passed through Q1 and fragmented to lose a specific neutral mass. MRM does not employ $m / z$ scanning and fixes Q1 and Q3 on specific $m / z$ values, ions at a specific $m / z$ value pass through $\mathrm{Q} 1$, fragment in q2 and a specific fragment passes 
through Q3. MRM is a highly sensitive scanning mode as the instrument duty cycle is focused on one or more specific ion-- ion fragment transitions rather than scanning for $\mathrm{m} / \mathrm{z}$ values that may not exist in the sample. MRM simply displays the intensity of the ion signal for each transition and therefore is most useful when coupled with chromatography. Lipids extracts are most commonly analyzed by product ion scans to help probe structural features (head group and fatty acyl chains), and by precursor ion scans to simplify complex biological samples to just specific lipid classes.

Figure 1.1.2 illustrates the general workflow for a lipidomics experiment initiated with lipid extraction leading to analysis via MS. The use of chromatography for further separation of lipid extracts prior to MS analysis is debated in the literature. ${ }^{14}$ Lipid extracts are complex and warrant chromatographic separation; however, complications with lipid chromatography are non-trivial.

In the case of glycerophospholipids, analyte molecules are amphipathic which means they possess properties that allow adsorption to both polar and non-polar surfaces. As a result, some literature suggests that direct infusion of complex lipid extracts into a mass spectrometer via electrospray ionization (ESI) is the best course of action. ${ }^{14}$ Direct infusion minimizes the exposure that a lipid sample has to capillary tubing leading up to ESI, decreasing sample loss and non-specific adsorption to sample handling surfaces. Direct infusion necessitates the need for high resolution MS to distinguish lipids of similar mass and literature has emerged that has demonstrated the analysis of thousands of lipids from a single extract via direction infusion high resolution MS. ${ }^{14}$

Some of the limitations of direct infusion how-ever include the fact that high resolution MS is not always available, and that many isobaric lipid species exist that may 
be chromatographically separable but are indistinguishable based on mass alone. Furthermore, chromatography offers the benefit of desalting a sample and reducing the number of analytes being simultaneously ionized via ESI. Salts such as sodium are prevalent in biological samples and persist in lipid preparations; the presence of salt will lead to signal splitting in the mass spectrum as lipid analytes may be protonated or cationized by a salt to achieve ionization. ESI operates on the principle that analytes are ionized based on having a higher proton affinity in comparison to the solvent within which they are contained; when samples are increasingly complex, ion suppression occurs as analytes of higher proton affinity are protonated while analytes of lower proton affinity are not and remain neutral. ${ }^{14}$ High performance liquid chromatography (HPLC or LC) remediates both problems. By binding lipid analytes to a stationary phase, salts are removed, and analytes are eluted in order of increasing hydrophobicity reducing sample complexity during the ESI process and remediating ion suppression. Furthermore, chromatographic separation concentrates analytes into a narrow elution window and therefore increases the sensitivity of analyses; quantitation is easily achieved by integrating the area under the peak produced from MS signal as the peak elutes from the column. These benefits are the main driving force behind the use of chromatography in most HPLC-ESIMS applications. However, the amphipathic nature of lipids causes considerable sample carryover that interferes with the precision of analyses and for this reason many research groups in this field have discontinued the use of chromatography. In light of this, we collected data to document the extent to which precision is hampered in HPLC-ESI-MS analyses of complex lipid samples and investigated novel chromatographic strategies to 
remediate these issues so that the fundamental benefits of using chromatography may be realized in the field of lipidomics.

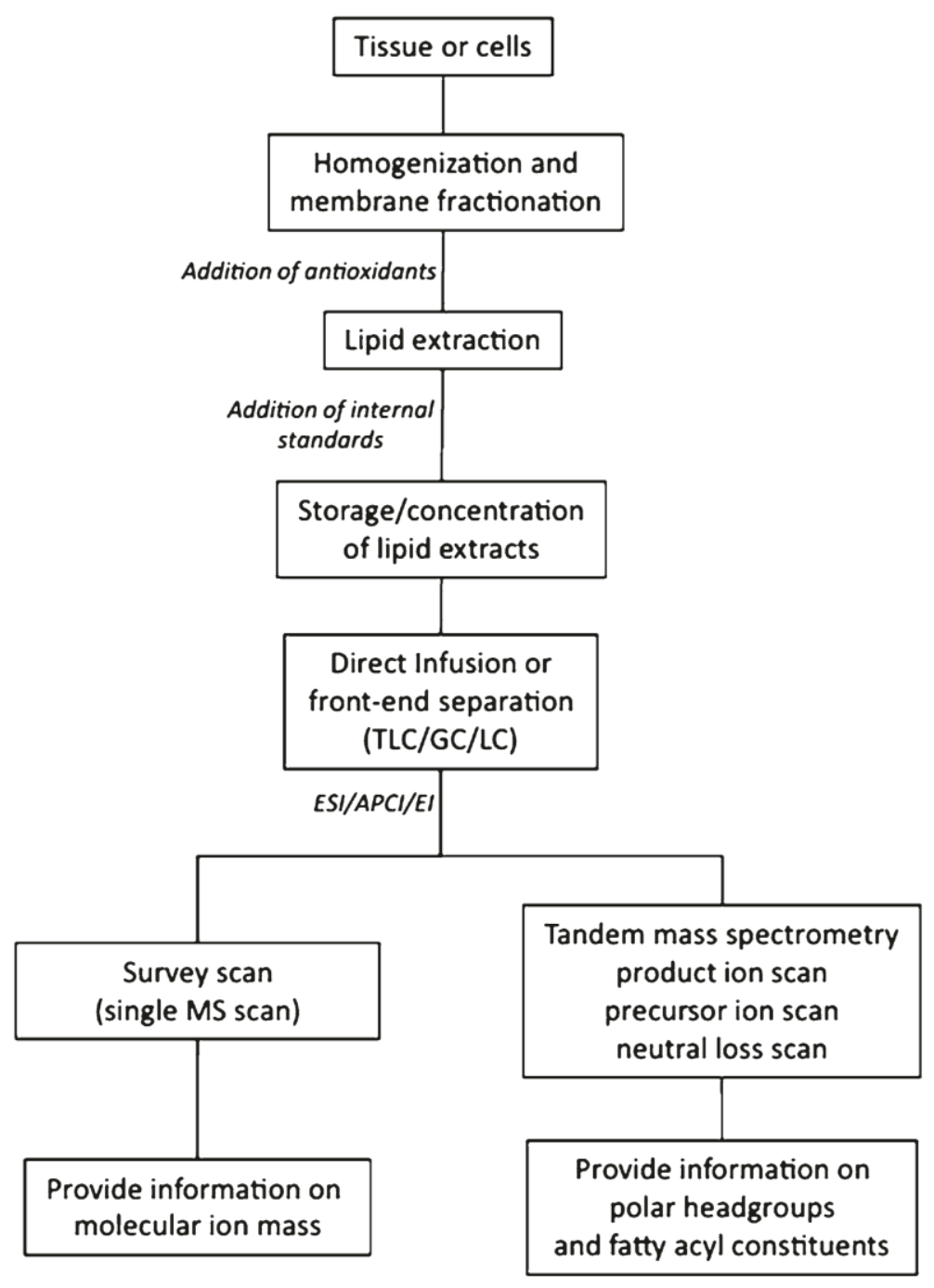

Figure 1.1.2 A General Workflow for Lipid Analysis ${ }^{13}$

\subsubsection{Optimization of Lipid Chromatography}

The vast majority of chromatographic separations are conducted using an octadecyl or $\mathrm{C}_{18}$ functionality covalently bonded to the stationary phase and a mixture of aqueous and less polar organic mobile phases to create a reversed phase separation. Within this chromatography scheme, analytes partition to the less polar stationary phase and are eluted 
either over time isocratically or when the eluent strength of the mobile phase is altered in a gradient to collapse the sample and elute it as a narrow band of eluate. The columns and hydraulic path of an HPLC are generally a mixture of stainless steel, polyetheretherketone and/or fused silica tubing. Therefore, lipid analytes are exposed to both polar surfaces within the hydraulic path as well as non-- polar surfaces on the stationary phase. In the case of glycerophospholipids, the separation capabilities of liquid chromatography are complicated by the amphipathic nature of lipid molecules as adsorption to any component of the hydraulic path via their polar head groups or hydrophobic tails is possible. To demonstrate this principle and the extent to which sample carryover is a result of polar interactions with the hydraulic path, we conducted several experiments where the column was removed in a reversed phase HPLC separation and sample carryover was observed, Fig. 1.1.3.

The four panels in Fig. 1.1.3 demonstrate that glycerophospholipids (PC and SM) have a propensity to adsorb to the hydraulic path of an HPLC. Although the elution time for all species is within the first $15 \mathrm{~min}$ of the solvent gradient (Fig.1.1.3a), the lipids had persisted despite several volumes of column washing following injection onto the empty column. The lipids shown in Fig. 1.1.3b are an average of the entire chromatographic run and demonstrate that dozens of phosphocholine-containing species elute from the column. The intensity of these peaks are roughly two orders of magnitude higher when examining an individual time point, their intensities have been diminished by averaging the signal at every $\mathrm{m} / \mathrm{z}$ over $60 \mathrm{~min}$. Following the acquisition of this data, a 20-min wash of $100 \%$ acetonitrile was flushed through the system. Figures $1.1 .3 \mathrm{c}$ and $1.1 .3 \mathrm{~d}$ repeat this experiment, injecting distilled water instead of lipid extract and demonstrate the extent of 
sample carryover despite injecting a blank and conducting a "column wash" (in quotations since there was no column present). These results demonstrate the extent to which polar lipids may adsorb to fused silica and stainless steel and highlight the challenge of separating amphipathic analytes. Interestingly, amphipathic cationic surfactants such as didodecyldimethylammonium are intentionally used to bind to the surface of fused silica in capillary electrophoresis (CE) and create a bilayer that reverses the charge on the wall of the capillary and therefore the flow of the electrolyte through the column. ${ }^{15}$ The parallels between this experimental practice in $\mathrm{CE}$ and the injection of polar lipids in a chromatographic system are striking; it is of little surprise that polar lipids such as PC and SM adsorb strongly to fused silica. The challenge in this result is that the methods that were used to obtain the data in Fig. 1.1.3 mirrored the most commonly used mobile phase system that appeared in the literature for lipid separation at the time. Due to these reasons the field as a whole had begun to move towards direct injection for MS analysis to avoid carryover issues. 


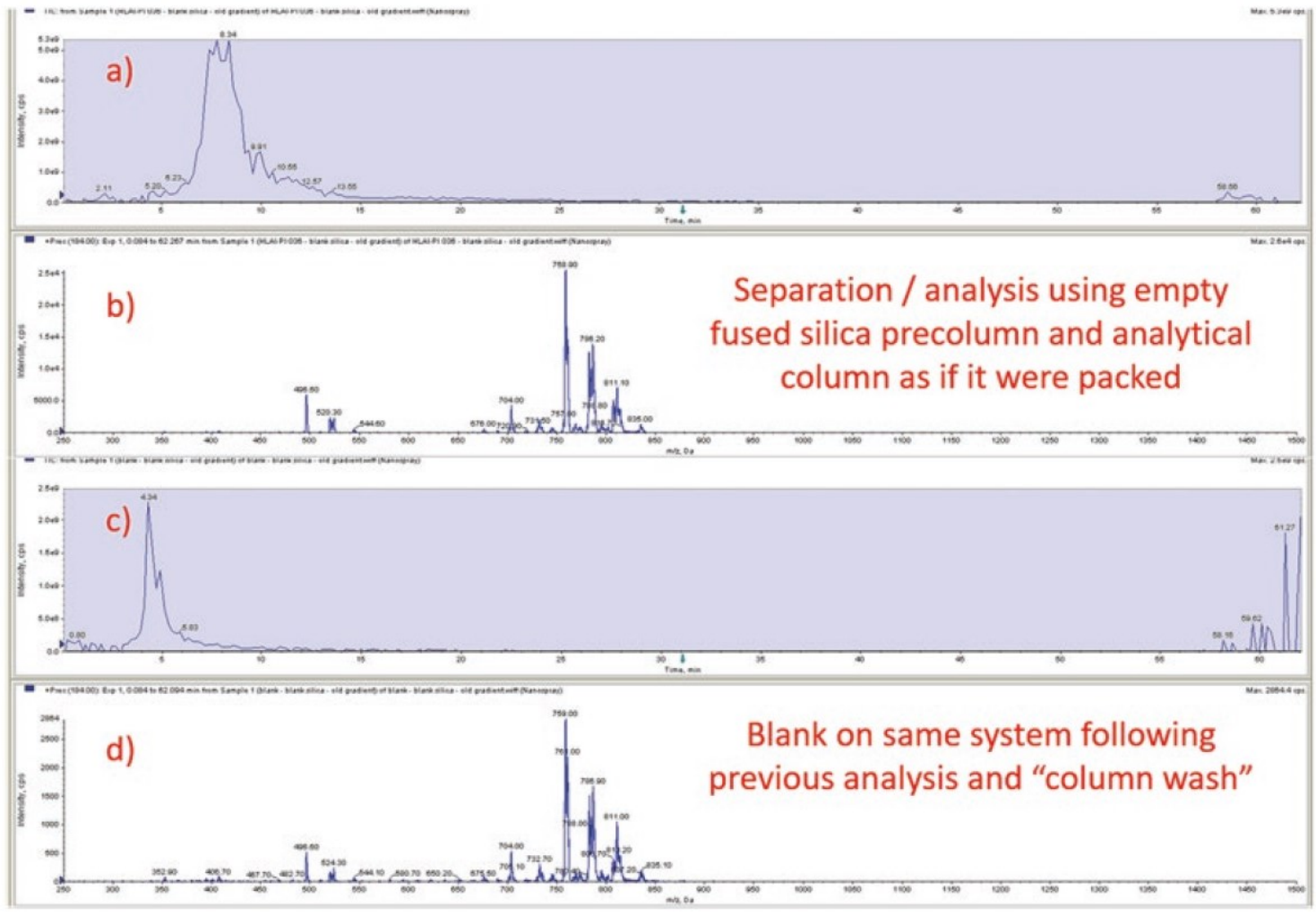

Figure 1.1.3 Observation of lipid carryover within an HPLC system that has no column attached. (a) the ion current over the $60 \mathrm{~min}$ from the initial injection of lipid extract, (b) the average precursor ion mass spectrum over the course of the entire run, (c) the ion current over a 60-min gradient after subsequently injecting 20 $\mu \mathrm{L}$ of water through the system, (d) the average precursor ion mass spectrum over the course of the entire blank run

Many mobile phase compositions were tested for their effect on chromatographic performance, all of them being a non-polar solvent containing a polar modifier. Ammonium-based salts were investigated for their effect on the system and it was determined that $10 \mathrm{mM}$ ammonium acetate was the most effective at disrupting hydrophilic interactions within the system. Higher concentrations of this salt did not yield an increase in performance and as the concentration was increased the -likelihood of flow stoppages increased as well, particularly in the ESI tip. It was also determined that $10 \mathrm{mM}$ ammonium 
acetate was beneficial to add to all mobile phases to continually disrupt polar interactions throughout the separation. Several modifications were made to the non-polar solvents that were used for analyte elution as well as system cleaning following analyses. The first modification was made following a series of experiments looking at the solubility of lipid extracts in aqueous buffers operating on the hypothesis that lipid analytes dissolved in ethanol injected into a column running aqueous buffers may render the lipids insoluble through the formation of micelles or aggregates. Although the nature of the insolubility was not determined, it was concluded that chromatographic performance diminished when the mobile phase solvents were composed of $70 \%$ water or greater. Furthermore, it was determined that no sample was lost via immediate elution at $70 \%$ water (not being retained by the column). Ultimately, it was deter-mined that the best mobile phase starting condition (buffer A) was $30 \%$ methanol in water with $10 \mathrm{mM}$ ammonium acetate. A variety of solvents were tested to elute lipid analytes including $100 \%$ acetonitrile or methanol, but the carryover was significant demonstrating insufficient eluent strength for these solvents. Solvents non-polar solvents were evaluated for lipid elution as well (e.g. hexanes) and it was observed that chromatographic elution was effective however ESI performance was deleteriously affected. Furthermore, the immiscibility of hexanes with aqueous buffers limited their use in a binary solvent system. Overall, we believe that the challenges associated with lipid chromatography are surmountable and that the benefits of chromatographic separation prior to ESI MS analysis may be realized using an optimized ternary solvent system 


\subsubsection{Chemical Derivatization to Improve Sensitivity in Lipidomics Analyses}

Chemical derivatization of lipids is one approach to increase sensitivity, separate isobaric masses, probe double bond location, and allow for methods of absolute quantitation of certain lipid classes. Here we review of some of the chemical derivatization techniques that have been developed to overcome challenges in MS analysis of lipids.

\subsubsection{Fatty Acyl Derivatization}

One of the earliest methods of chemical derivatization of fatty acids is methylation followed by GC-MS analysis. Methylation of fatty acid lipids (Scheme 1.1.2), improves gas chromatographic properties such as volatility and polarity. ${ }^{16}$ The three main methylation derivatization procedures are acid catalyzed Fischer esterification, alkylation via diazomethane and base catalyzed transesterification. Briefly, acid catalyzed esterification and base catalyzed transesterification relies on a large excess of methanol to push the equilibrium to completion, while alkylation via diazomethane proceeds irreversibly to the corresponding fatty acid methyl ester (FAME).

A modern example of preparing fatty acid methyl esters (FAMEs) to improve their chromatographic separation is shown below (Fig. 1.1.4). This experiment uses methanolic potassium hydroxide to transesterify the lipids extracted from eggs. ${ }^{17}$ Figure 1.1.4 (left) shows the gas chromatograph of several fatty acid methyl esters, which are denoted by number of carbons in the chain and number of unsaturations in the chain. Figure 1.1.4 (right) shows an electron impact MS of methyl palmitate (16:0) that was successfully derivatized, extracted and separated via gas chromatography (GC). This is a standard 
chemical derivatization technique to improve the gas chromatographic properties of fatty acyl components.

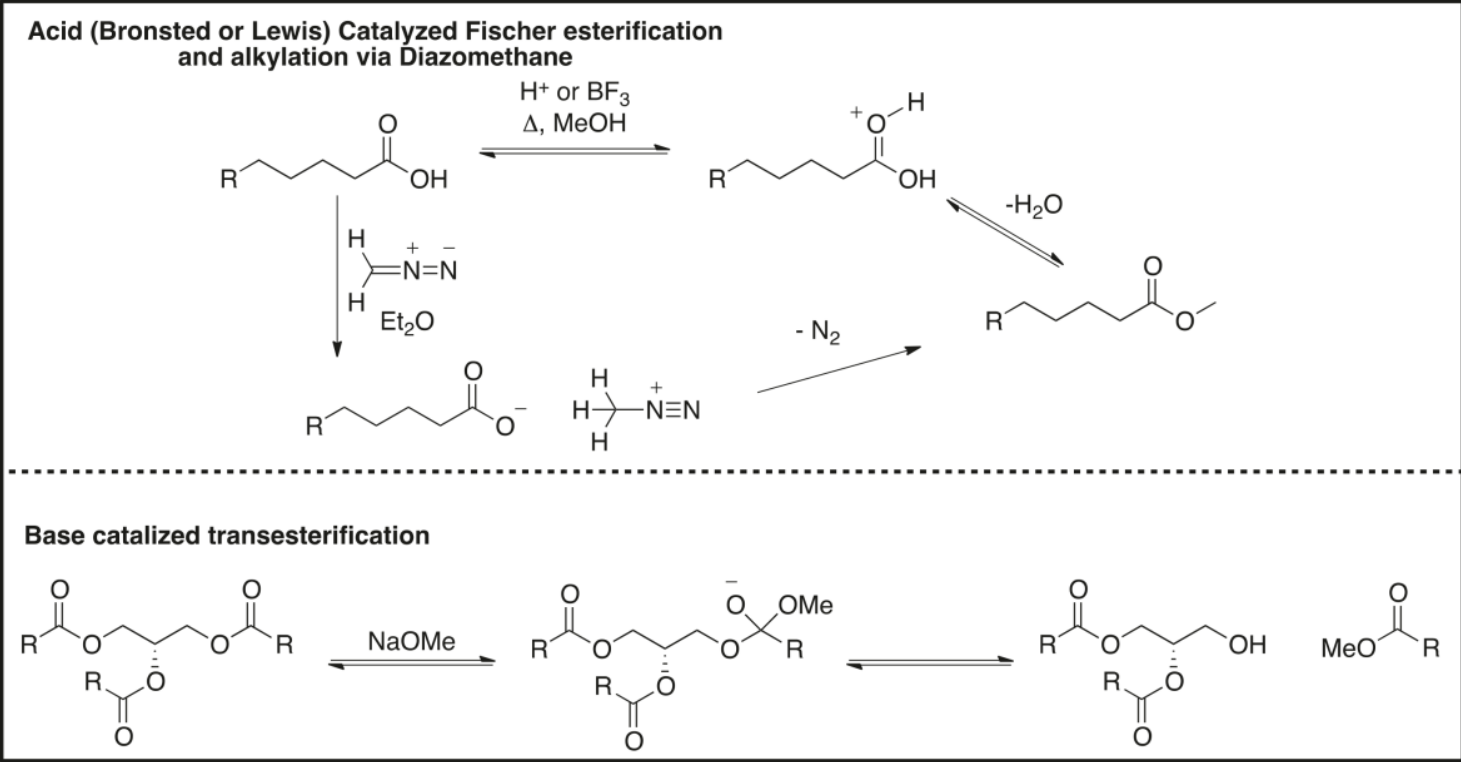

\section{Scheme 1.1.2 Synthesis of Fatty Acid Methyl Esters}
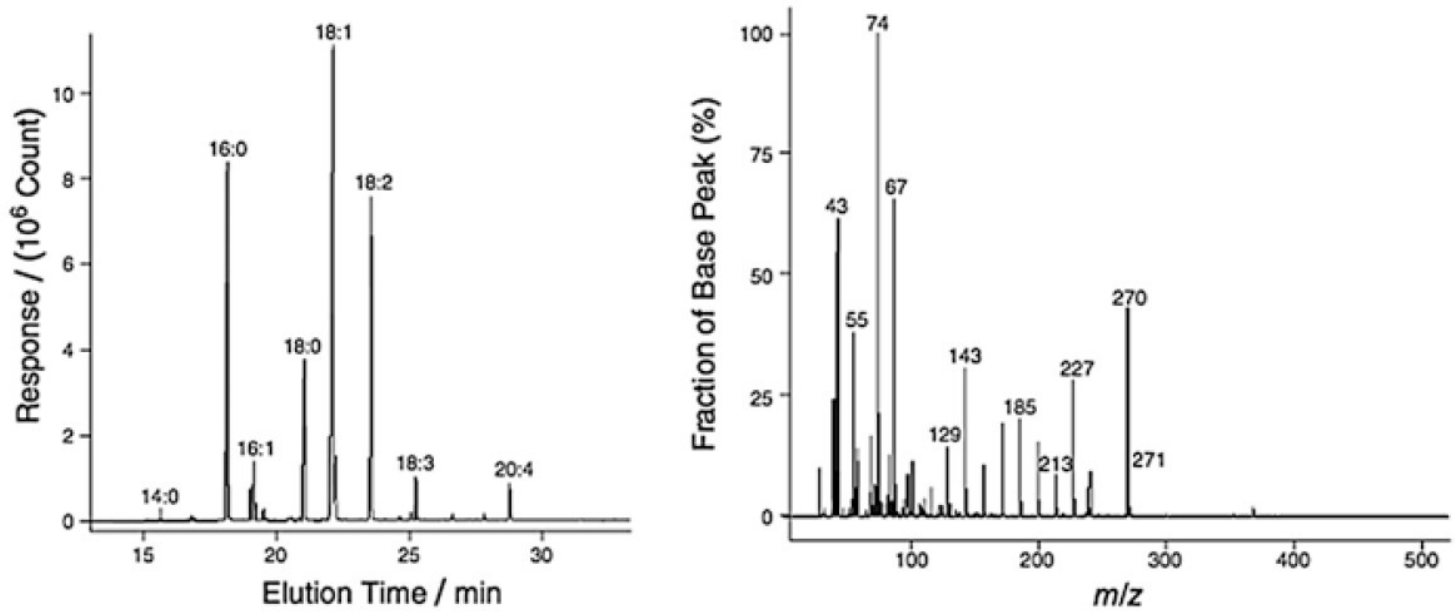

Figure 1.1.4 Gas Chromatograph of FAMEs Derived from Eggs (left) and EI-MS of Methyl Palmitate (right) ${ }^{17}$ 
Han et al. have also employed a novel chemical derivatization technique for fatty acyls. The first example is the derivatization of 4-hydroxyalkenals to improve their quantitation from complex biological samples. ${ }^{18}$ 4-hydroxyalkenals are known lipid peroxidation metabolites that can be used as lipid biomarkers for estimating the level of oxidative stress within a biological sample. However, the analysis of these compounds by MS is relatively difficult since they readily form adducts with proteins and peptides from cell extracts. The general reaction scheme and mechanism is shown below (Scheme 1.1.3), the 4-hydroxylalkeanl is incubated at $37{ }^{\circ} \mathrm{C}$ in water for $24 \mathrm{~h}$ before a microscale liquidliquid extraction. These adducted products could then be analyzed using a triple quadrupole mass spectrometer. Each of the samples also contained 4-hydroxynoneneal- $d_{3}$ as an internal standard where the deuterium is added to give a mass shift of $3 \mathrm{Da}$ to avoid complicating the spectra.

After analysis of 4-hydroxyalkenal standards using product ion scanning, it was observed that the carnosine moiety had extremely reliable fragmentation. The loss of 17 , 63, 71, and 117 Da corresponded to the neutral loss of ammonia, carbon dioxide plus ammonia, carbonyl chain (fragmentation at amide bond), and carbonyl chain plus carbon dioxide respectively. It was noted by the experimenters that the same fragments occurred with the deuterated standard as with the non-deuterated compound and thus it could be used as a relative standard for quantitation purposes. Figure 1.1.5 illustrates the results of neutral loss scans from derivatized 4-hydroxyalkenals from rat liver. By forming carnosine adducts, four different 4-hydroxyalkenals could be observed and precisely quantified by the requisite neutral loss scans. 


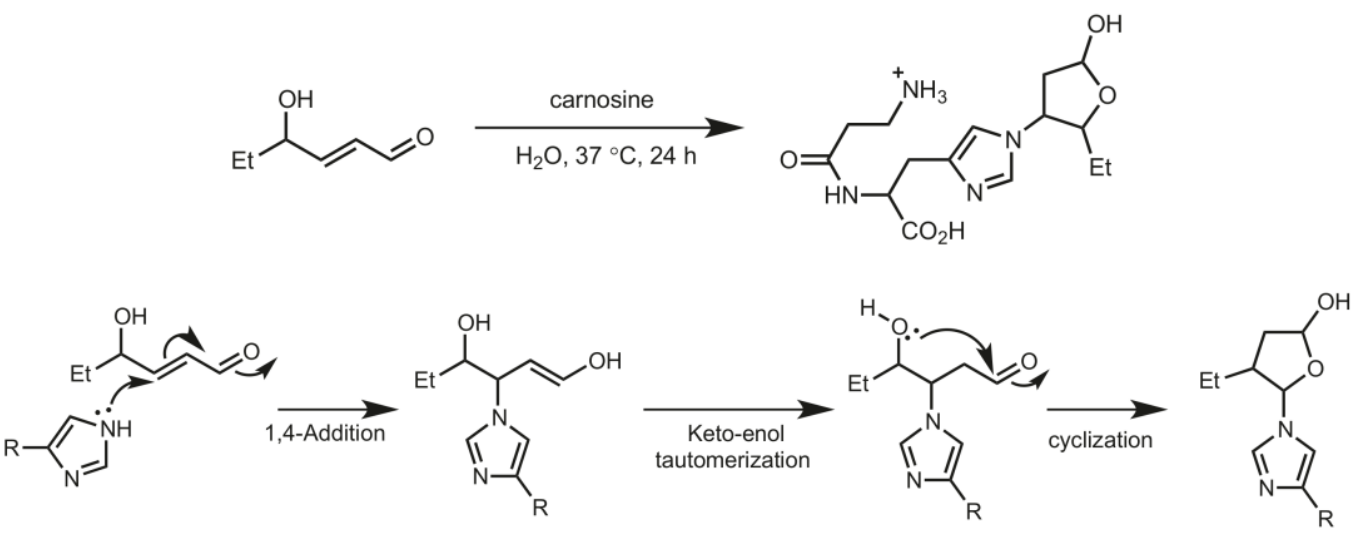

Scheme 1.1.3 General Reaction Scheme and Mechanism of Adduct Formation of 4hydroxyalkenals and Carnosine

A NLS71

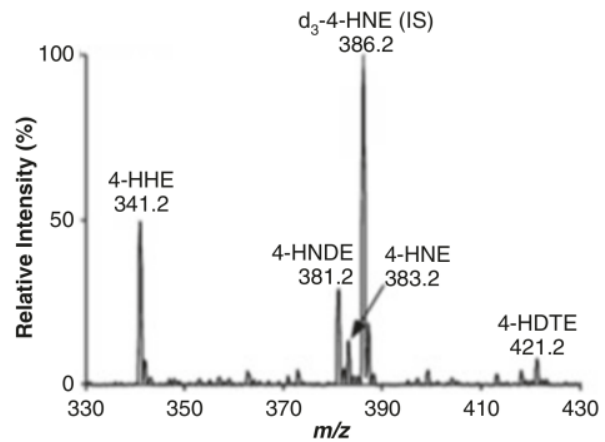

C NLS17

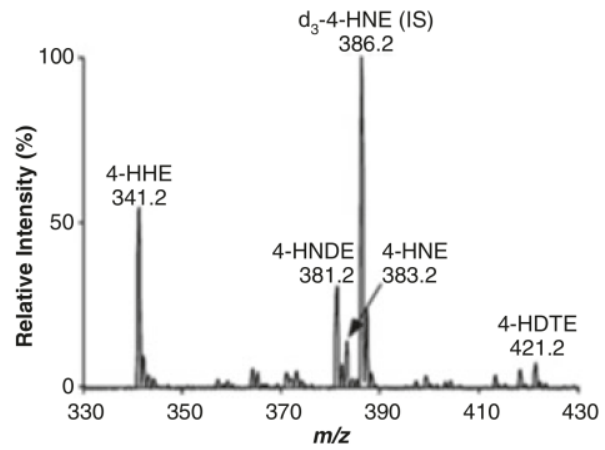

B NLS117

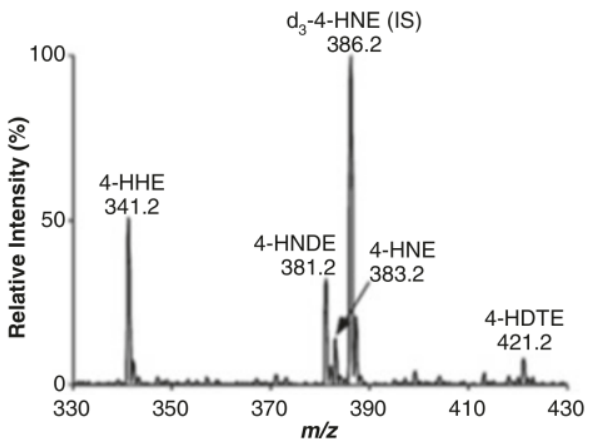

D NLS63

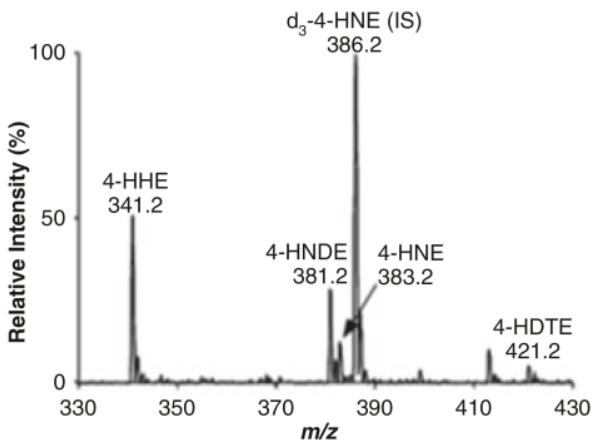

Figure 1.1.5 Quantitation of a Variety of 4-hydroxyalkenals Using Neutral Loss

Scanning Mode ${ }^{18}$ 


\subsubsection{Di- and Triacylglycerol Derivatization}

The analysis of di- and triacylglycerols is important as these lipids are some of the most ubiquitous classes found in mammalian biology. The primary function of these lipids is energy storage and they often serve as precursors to more complex lipid classes (e.g. glycerophospholipids); however, they can play roles in signal transduction pathways as well. ${ }^{13}$ The elucidation of absolute lipid structure (location of the unsaturations and position of fatty acyl chains with respect to $s n-1$ and $s n-2)$ is an obstacle that cannot be entirely surmounted by MS alone highlighting the importance of breakthroughs in chemical derivatization in this area.

In 2010 Blanksby et al. devised a chemical derivatization technique to help elucidate the absolute structures of triacylglycerols via gas phase ozonation (Scheme 1.1.4). ${ }^{19}$ Using a modified linear ion trap, methods were developed to use ozone-induced dissociation (OzID) to yield characteristic fragments that indicated the position of the double bonds within fatty acyl chains. Ozone was generated externally from $\mathrm{O}_{2}(\sim 11 \%$ by volume) and was flowed into the collision cell along with nitrogen gas at pressures of $\sim 10^{-3}$ Torr. Ions that were generated from a lipid solution in methanolic sodium acetate were trapped in the collision cell for 10-25 ms before being analyzed by the final quadrupole to generate product ion mass spectra. 


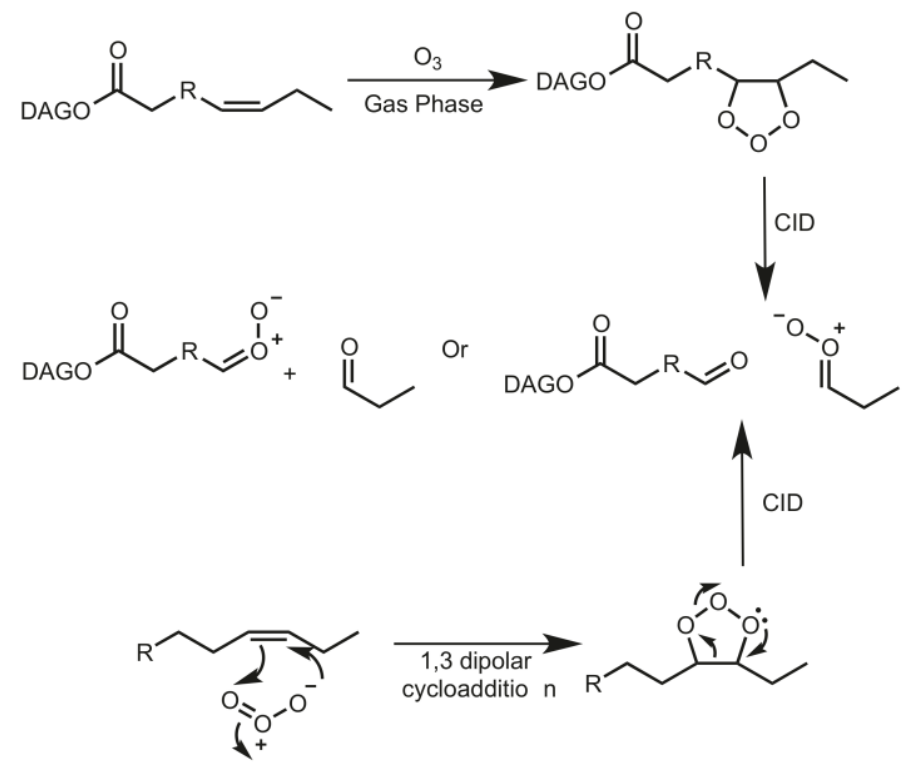

\section{Scheme 1.1.4 General Reaction and Mechanism of the Ozonolysis of Triacylglycerols}

A triacylglycerol standard was used to optimize collision energies to fragment the primary ozonide into two main products, an aldehyde and carbonyl oxide fragment. Both fragments can be used to determine double bond location since ozone will selectively react with an alkene in the lipid and predictable mass losses will ensue. The collision energy was adjusted until all of the primary ozonide was fragmented (Fig. 1.1.6). From the mass losses of $158\left(\mathrm{C}_{9} \mathrm{H}_{18} \mathrm{O}_{2}\right)$ and $142\left(\mathrm{C}_{9} \mathrm{H}_{18} \mathrm{O}\right) \mathrm{Da}$ from the primary ozonide, the location of the unsaturation was determined to be at $C 9$ ( $\omega-9$ unsaturated fatty acyl). Using this method of chemical derivatization, predictable fragmentation of OzID is a useful tool in the structural elucidation of triacylglycerols. 

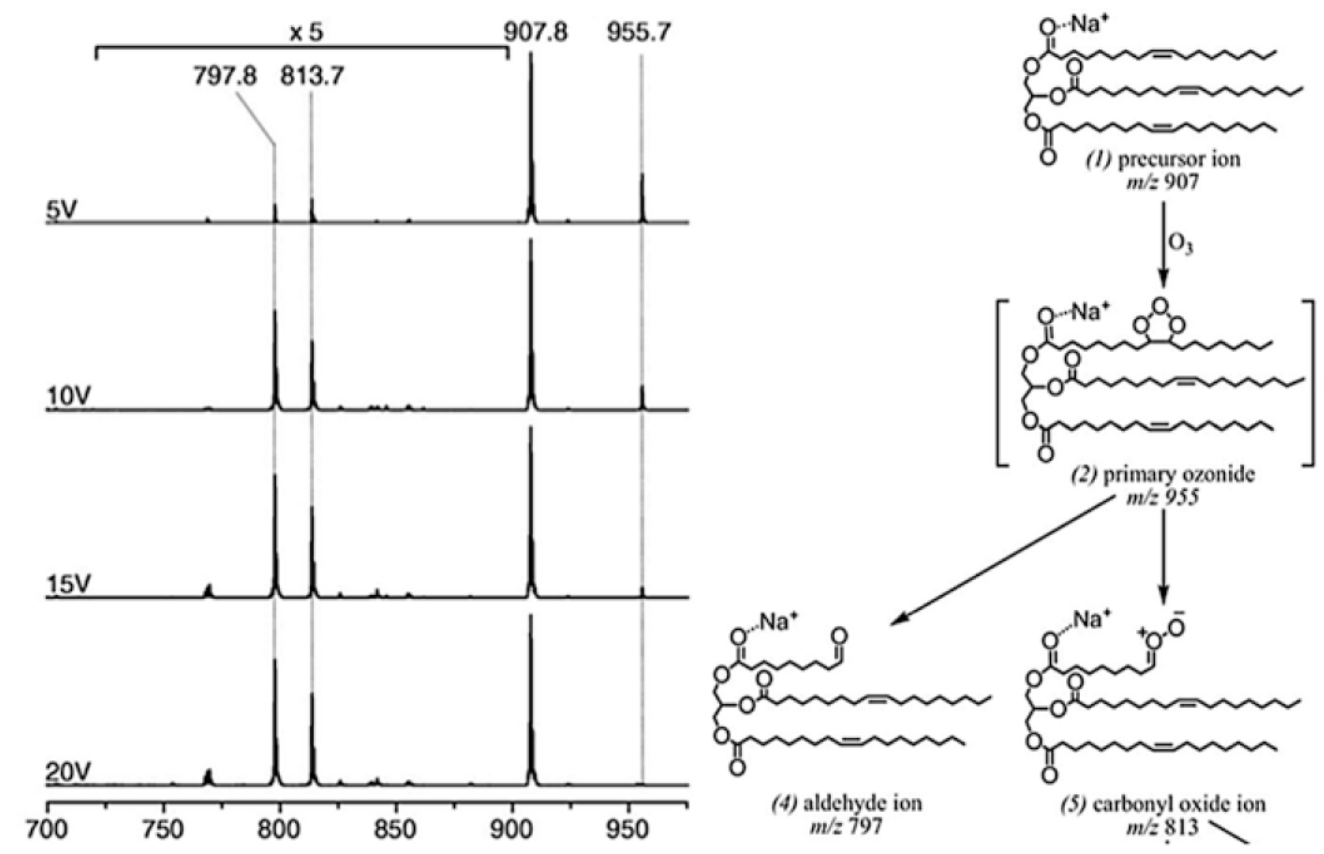

Figure 1.1.6 Optimization of Collision Energy for OzID (left) Possible Products of

\section{OzID (right)}

Although diacylglycerols (DAG) play very important roles in biological mechanism, their quantitation has been challenging due to low abundance and poor proton affinity. ${ }^{20,21}$ An example of chemical derivatization of acylglycerols is presented by Han et al. in 2014, where the use of a common peptide coupling reaction (esterification/amidation) is used to allow for enhanced ESI-MS ionization efficacy. ${ }^{22}$ The general reaction conditions and mechanism are shown in Scheme 1.1.5, whereby adding a tertiary amine to the DAG, a large increase in proton affinity (ionization efficiency) was observed. The MS analysis was performed on a triple quadrupole instrument to provide product, precursor and neutral loss scans.

Using an equimolar amount of 16 different DAG standards at fmol $/ \mu \mathrm{L}$ concentrations, this group showed extremely favorable fragmentation of dimethylglycine 
(DMG) modified DAG that gave a reproducible ion at $\mathrm{m} / z 110$ corresponding to lithiated DMG. This predictable fragmentation could then be used in precursor and neutral loss scans to simplify complex biological samples for relative quantitation of many DAG species. Figure 1.1.7 shows the results of both protonated and lithiated neutral loss scans. The spectrum on the right illustrates all peak intensities are approximately equal which is advantageous for the quantitation of each of the lipid species; the spectrum on the left however (using LiOH to initiate the ionization), showed a variety of peak intensities. The reason for using a lithium additive is that lithium facilitates fatty acyl cleavage upon CID and thus some structural information is obtainable. In summary, this chemical derivatization method greatly increases the ionization efficiency of DAGs, permitting quantitation when formic acid or structural elucidation when $\mathrm{Li}^{+}$are added respectively.

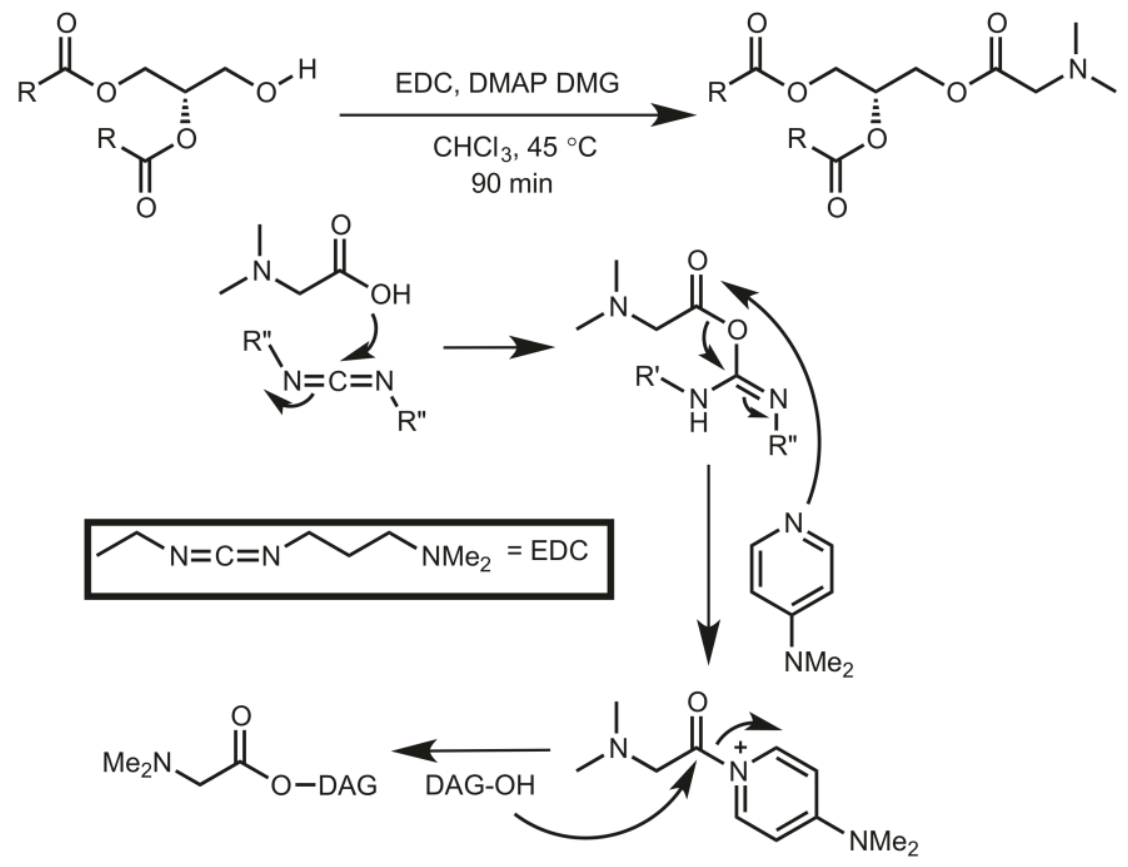

Scheme 1.1.5 Esterification of Diacylglycerols 

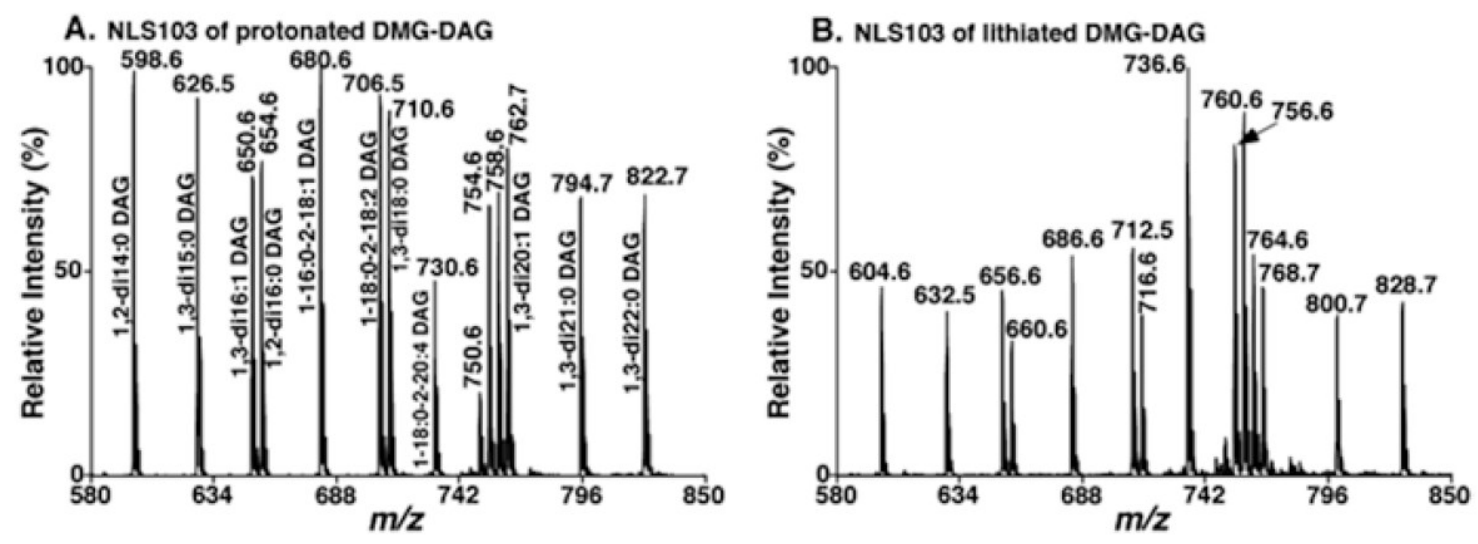

Figure 1.1.7 Neutral Loss of 103 Da of an Equimolar Mixture of DAG Standards ${ }^{22}$

\subsubsection{PE and PC Derivatization}

As described above, PE and PC constitute most of the total lipids in a cell and therefore require rigorous analytical methods. Challenges encountered when analyzing PE and PC include sensitivity loss via ion suppression in ESI and isobaric interference. For example, a PC with fatty acyl chains of 16:0 and 22:6 would have the exact same $\mathrm{m} / \mathrm{z}$ value as a PC with 18:1 and 20:5. The structure elucidation of PE and PC is crucial to fully understand lipid biochemistry and the roles they play in cellular life highlighting the need and importance of chemical derivatization techniques in this area.

The Blanksby group used their OzID methodology to analyze PCs from lipids extracted from cow brain. ${ }^{19}$ Figure $1.1 .8 \mathrm{~b}$ illustrates the resulting spectrum when OzID is used on a lipid observed at 782.6 Da. Two fragmentation products are observed for each structural isomer, where the higher mass fragment corresponds to a sodiated carbonyl oxide and the lower mass fragment corresponds to a sodiated aldehyde fragment. The peaks denoted with a diamond are directly from $\omega-7$ and $\omega-9$ oxidative cleavage where peaks marked with asterisks are from the loss of 59 Da corresponding to neutral loss of $\mathrm{NMe}_{3}$ 
from the choline head group. In this way, the location of the double bond within fatty acyl substituents can be obtained as well as the differentiation of isobaric PC species.

(a)

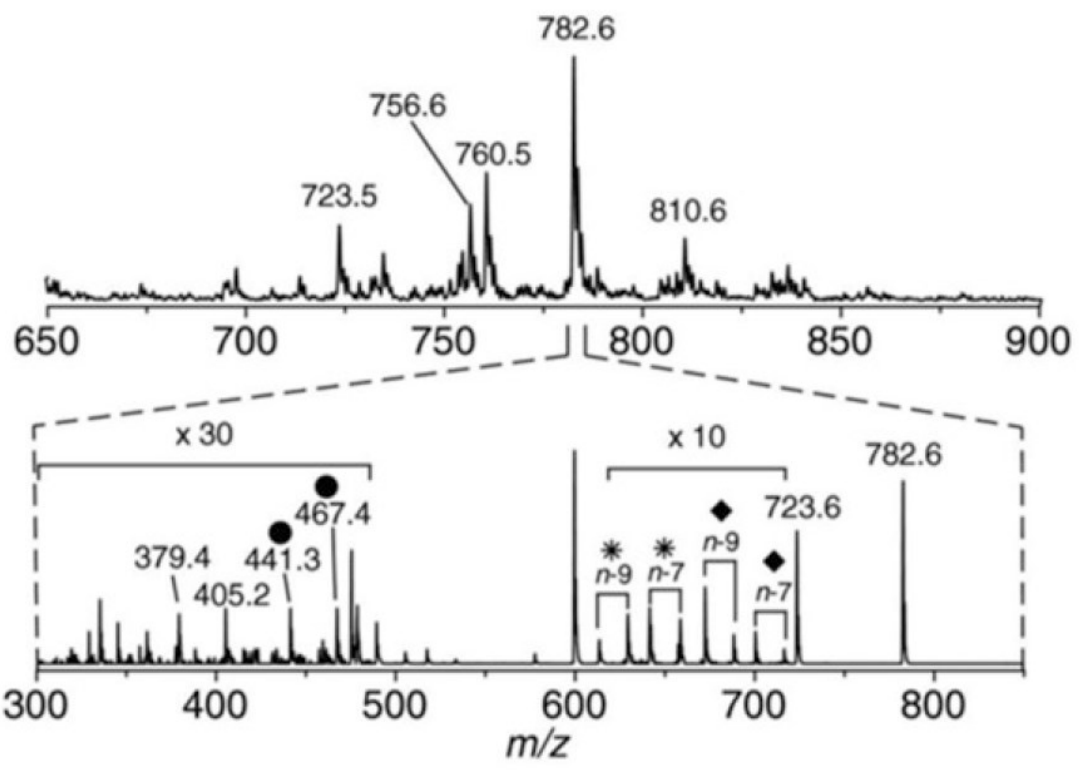

Figure 1.1.8 OzID of PCs from Cow Brain ${ }^{19}$

PE lipids have a free amine (at higher $\mathrm{pH}$ ) that can act as a nucleophile for activated electrophiles such as acid chlorides and $N$-hydroxy succinimide (NHS) esters. In 2012, the Reid group developed a novel chemical derivatization procedure for PEs based on a common nucleophilic substitution of an activated NHS ester (Scheme 1.1.6) ${ }^{23}$ Interestingly, $d_{6}$-dimethylthibutanoylhydroxysuccinimide ester ( $d_{6}$--DMBNHS) bears a fixed positive charge in the form of a sulfonium ion. Therefore, when $d_{6}$-DMBNHS is reacted with PE species to form a new amide bond, the lipid will also bear a fixed positive charge improving its ionization efficiency greatly. This will greatly increase the sensitivity of the lower abundance PE species as well as separating the isobaric lipid species due to the mass shift of the $d_{6}$-DMB moiety (136 Da). Again, some of the challenges with quantitation and 
isobaric lipid species can be overcome by a facile one step chemical derivatization procedure.

This group was interested in obtaining the global lipid profile of metastatic colon adenocarcinoma cell lines by using their novel technique to identify and quantify some low abundant PE species as well as the more abundant ones. Figure 1.1.9 illustrates the results of a non-derivatized lipid extract and $d_{6}$-DMB modified lipid extract from SW480 cancer cell lines. The emergence of higher mass peaks as well as the decrease in intensity of many of the residual peaks from the unmodified lipid extract indicates that a significant number of isobaric lipid species were separated. Many of the modified lipids were identified and accurately quantified using high-resolution/accurate-mass analysis, permitting a global lipid comparison of the two cancer cell lines. This information can be used to monitor the differences between non-cancerous and metastatic cell and perhaps be used to help identify colon cancer at its early stages.

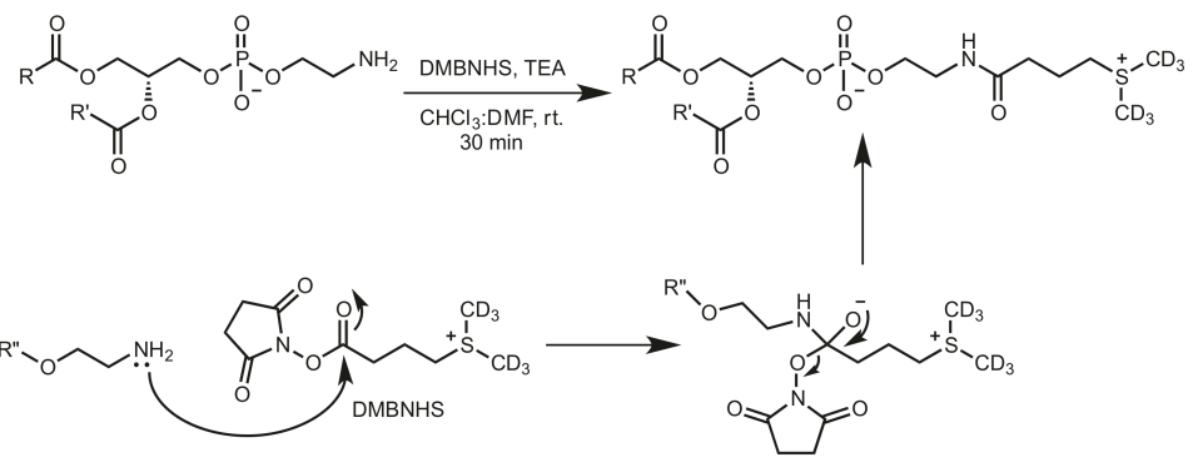

Scheme 1.1.6 Chemical Derivatization of PEs with $d_{6}$-DMBNHS General Reaction and Mechanism 


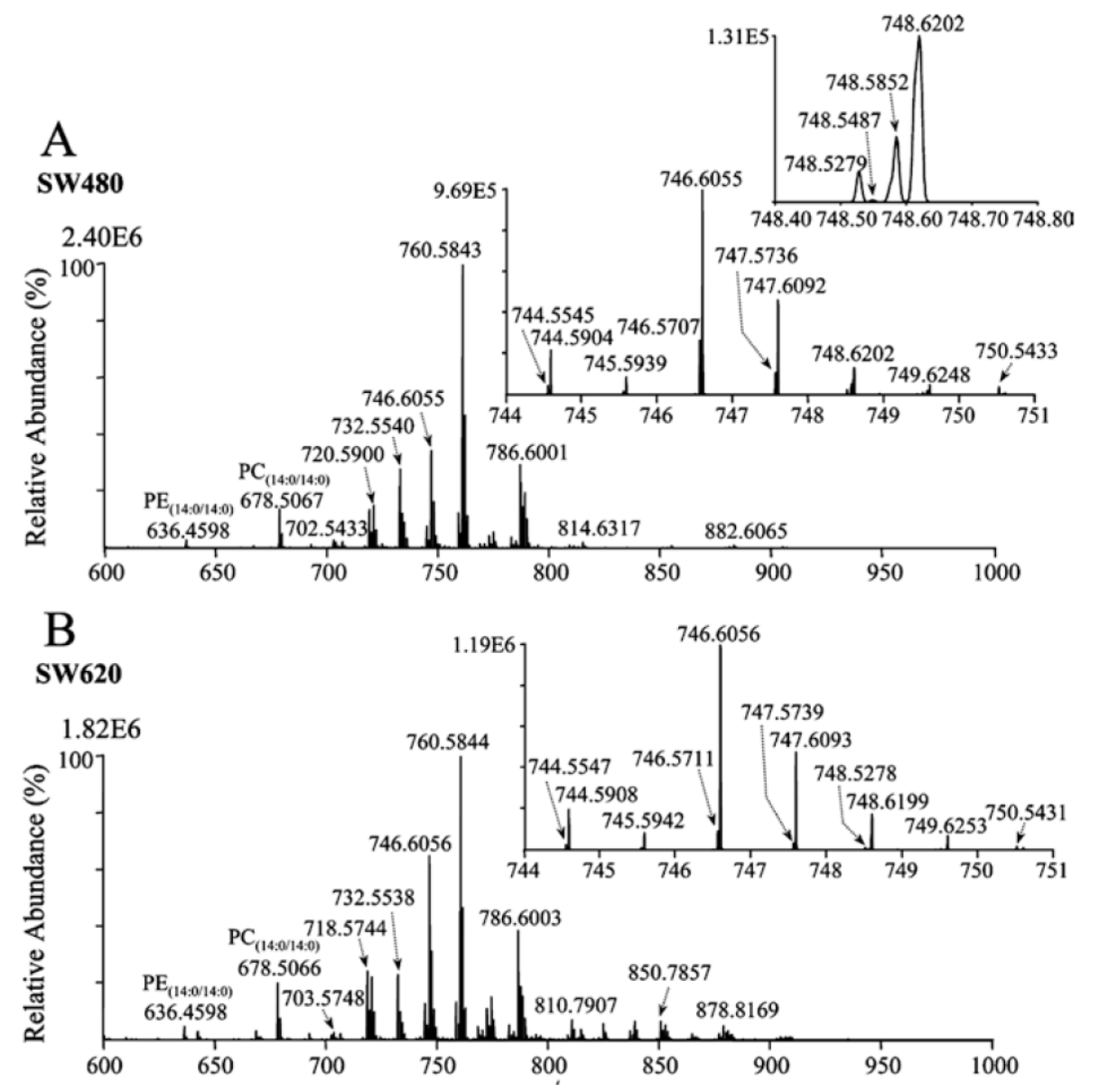

Figure 1.1.9 Unmodified lipid extract (top) and $d_{6}$-DMB modified lipid extract

$(\text { bottom })^{23}$

Other modifications of PE and PC head group can be accomplished by methylation via an activated methyl electrophile. Some examples of methyl electrophiles used to alkylate the amine moiety of ethanolamine phospho-lipids includes, methyl iodide ${ }^{24}$ and reductive amidation with formaldehyde/sodium cyanoborohydride. ${ }^{25}$ Our group has developed the used of acid-initiated diazomethane (DZM) alkylation as a simple, fast, and cost-effective method of PC and PE alkylation entitled trimethylation enhancement using diazomethane (TrEnDi) is highlighted in Scheme 1.7. ${ }^{26}$ This method is observed to 
methylate primary amines as well as the phosphate oxygen giving a truly permanent positive charge residing on derivatized lipid. The scheme below shows the general reaction of diazomethane with PE. This reaction requires the use of an acid to mediate the eventual protonation of diazomethane leading to the loss of $\mathrm{N}_{2}$ Gas.

The signal of unmodified PE/PC lipids can be split between protonated and sodiated species that inevitably decreases sensitivity as well as further complicating spectra. The methylation of PE and PC via TrEnDi requires the use of an acid with a non-coordinating counter ion because the counterion can react with the DZM in an unsatisfactory way. This type of modification is very efficient in that, the reactions go to $99 \%$ completion as well as only producing $\mathrm{N}_{2}$ and the sole byproduct. Thus, a post extraction protocol is not required.

The reaction of diazomethane with PE and PC lipid standards yielded a significant increase in sensitivity (Table 1.1.1). This increase was measured by comparing the relative intensities of modified lipid with non-modified in the same sample solution (i.e. the modified and non-modified samples were mixed in an equimolar ratio before analysis). It was observed that protonated PE shows a 625 -fold sensitivity increase, and sodiated PE shows a 2.2-fold increase. For PC the results were similar where protonated and sodiated PC gave 145- and 1.04-fold increases. These results were the first of their kind in the chemical derivatization of glycerophospholipids.

More recently, we have developed a strategy using ${ }^{13} \mathrm{C}$-labelled diazomethane to isotopically label glycerophospholipids via TrEnDi, termed ${ }^{13} \mathrm{C}-\mathrm{TrEnDi} .{ }^{27}$ This modification to the derivatization remediates the isobaric overlap of PE and PC lipids with identical acyl chains (Scheme 1.1.8). The utility of this derivatization was demonstrated on a lipid extract from HeLa cells where the number of PE lipids observed above the limit 
of detection almost doubled and several phosphatidylserine lipids were observed that were previously invisible to MS analysis.<smiles>[R]C(=O)OC[C@H](COP(=O)([O-])OCCN)OC([R])=O</smiles><smiles>[R6][PH](=O)([O-])OCCN</smiles><smiles>[R]C(=O)OC[C@H](COP(=O)(OC)OCC[N+](C)(C)C)OC([R])=O</smiles>

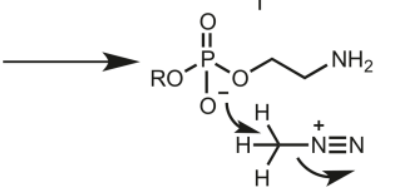

Scheme 1.1.7 Reaction of Diazomethane with PE General Reaction and Mechanism

Table 1.1.1 Sensitivity Comparison of Methylated PE and PC Against Non-Modified

$$
\operatorname{lipids}^{26}
$$

\begin{tabular}{|c|c|c|c|c|c|c|}
\hline \multirow[b]{2}{*}{ Lipid } & \multicolumn{3}{|c|}{ Unmodified } & \multicolumn{3}{|c|}{ TrEnDi-modified } \\
\hline & Species & $m / z(\mathrm{Th})$ & $\begin{array}{l}\text { Relative } \\
\text { intensity (\%) }\end{array}$ & $\begin{array}{l}{\left[\mathrm{M}^{\mathrm{Tr}}\right]^{+}} \\
(\mathrm{Th})\end{array}$ & \begin{tabular}{|l|} 
Relative \\
intensity (\%)
\end{tabular} & $\begin{array}{l}\text { Sensitivity } \\
\text { increase }^{a}\end{array}$ \\
\hline \multirow[t]{3}{*}{ PE (16:0/18:1(9Z)) } & {$[\mathrm{M}+\mathrm{H}]^{+}$} & 718.52 & 0.16 & 774.59 & 100 & $625 x$ \\
\hline & {$[\mathrm{M}+\mathrm{Na}]^{+}$} & 740.52 & 45.0 & & & $2.22 x$ \\
\hline & sum & & 45.2 & & & $2.21 \times$ \\
\hline \multirow{4}{*}{$\begin{array}{l}\text { PS } \\
(18: 0 / 18: 2(9 Z, 12 Z))\end{array}$} & {$[\mathrm{M}+\mathrm{H}]^{+}$} & 788.59 & 0.09 & 858.67 & 100 & $1110 x$ \\
\hline & {$[\mathrm{M}+\mathrm{Na}]^{+}$} & 810.59 & 1.16 & & & $86.2 \times$ \\
\hline & $\begin{array}{l}{[\mathrm{M}-\mathrm{H}+} \\
2 \mathrm{Na}]^{+}\end{array}$ & 832.57 & 1.84 & & & $54.3 \times$ \\
\hline & sum & & 3.09 & & & $32.4 x$ \\
\hline \multirow[t]{3}{*}{ PC (18:1(9Z)/14:0 } & {$[\mathrm{M}+\mathrm{H}]^{+}$} & 732.56 & 0.69 & 746.57 & 100 & $145 \times$ \\
\hline & {$[\mathrm{M}+\mathrm{Na}]^{+}$} & 754.54 & 96.2 & & & $1.04 x$ \\
\hline & sum & & 96.9 & & & $1.03 \times$ \\
\hline \multirow[t]{3}{*}{ SM (d18:1/16:0) } & {$[\mathrm{M}+\mathrm{H}]^{+}$} & 703.60 & 3.79 & 717.60 & 100 & $26.4 \times$ \\
\hline & {$[\mathrm{M}+\mathrm{Na}]^{+}$} & 725.58 & 36.1 & & & $2.77 \times$ \\
\hline & sum & & 39.9 & & & $2.51 \times$ \\
\hline
\end{tabular}

${ }^{a}$ Fold increase calculated by dividing the relative intensity of the TrEnDi-modified lipid species to the relative intensity of the unmodified lipid species 


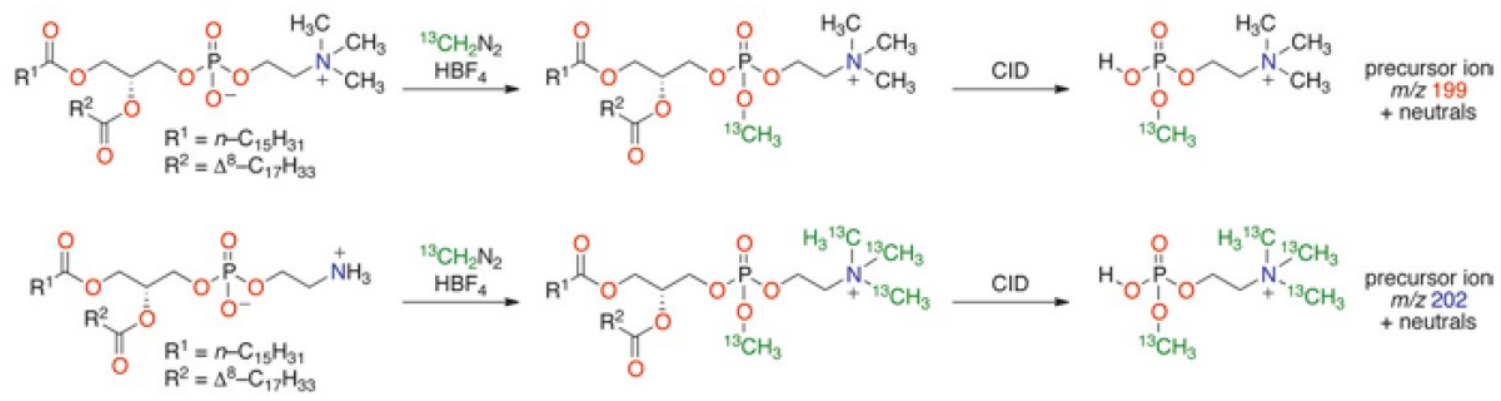

Scheme 1.1.8 ${ }^{13} \mathrm{C}-T r E n D i$ derivatization of PC(16:0/18:1) and PE(16:0/18:1)

\subsubsection{Conclusion}

Through careful optimization, the benefits of chromatography may be applied to the separation of complex lipid samples. Using a ternary gradient, the precision of lipid separations is maintained with good chromatographic peak shape and minimal carryover. Deeper insight into lipid biochemistry is empowered via chemical derivatization techniques that help overcome challenges in analyzing lipids by MS. Derivatization techniques increase sensitivity, separate isobaric masses, and elucidate absolute structure of a variety of different lipid classes. Successful derivatization techniques are typically simple organic reactions performed on a very small scale and are effective on standard solutions as well as complex lipid samples. Early indicators in the literature reveal that additional developments in the chemical derivatization of lipids will continue to emerge in this field as the importance of lipids in cellular biochemistry becomes more fully realized.

\subsubsection{References}

1. Wenk, M. R. Nat. Rev. Drug. Discov. 2005, 4, 594-610.

2. Shimizu, T. Annu. Rev. Pharmacol. Toxicol. 2009, 49, 123-150.

3. Meyer, H.D.; Jakobs, K. H. Biochim. Biophys. Acta. 2007, 1768, 923-940. 
4. Kirschnek, S.; Paris, F.; Weller, M.; Grassme, H.; Ferlinz, K.; Riehle, A.; Fuks, Z.; Kolesnick, R.; Gulbins, E. J. Biol. Chem. 2000, 275, 27316-27323.

5. Lemmon, M.A. Nat. Rev. Mol. Cell Biol. 2008, 9, 99-111.

6. Meer, G. EMBO J. 2005, 24, 3159-3165.

7. Horton, H.R.; Moran, L.A.; Scrimgeour, K.G.; Perry, M.D.; Rawn, J.D. (2006) Principles of biochemistry, 4th edn. Pearson, Upper Saddle River, pp 479-509.

8. Pulfer, M.; Murphy, R.C.; Mass. Spectrom. Rev. 2003, 22, 332-364.

9. Bartke, N.; Hannun, Y. J. Lipid Res. 2009, 50, S91-S96.

10. Goldfine, H. Prog. Lipid Res. 2010, 49, 493-498.

11. Bligh, E.G.; Dyer, W.J. Can. J. Biochem. Physiol. 1959, 37, 911-917.

12. Folch, J.; Lees, M.; Sloane-Stanley, G.H. J. Biol. Chem. 1957, 226, 497-509.

13. Kalil, M.B.; Hou, W.; Zhou, H.; Elisma, F.; Swayne, L.A.; Blanchard, A.P.; Yao, Z.; Bennett, S.A.; Figeys, D. Mass Spectrom. Rev. 2010, 29, 877-929.

14. Yang, K.; Han, X. Trends Biochem. Sci. 2016, 41, 954-969. b) Wu, X.; Oleschuk, R. D.; Cann, N. M. Analyst 2012, 137 (18), 4150-4161. c) Konermann, L.; Ahadi, E.; Rodriguez, A. D.; Vahidi, S. Anal. Chem. 2013, 85 (1), 2-9

15. Harris, D.C. (2016) Quantitative chemical analysis, 9th edn. W. H. Freeman and Co., New York

16. Christie, W.W. Preparation of ester derivatives of fatty acids for chromatographic analysis. Adv Lipid Methodol - Two 1993, 2, 69-111.

17. Alty, L.T. J. Chem. Educ. 2009, 86, 962-965.

18. Wang, M.; Fang, H.; Han, X. Anal. Chem. 2012, 84, 4580-4586. 
19. Poad, B.L.; Pham, H.T.; Thomas, M.C.; Nealson, J.R.; Campbell, J.L.; Mitchell, T.W.; Blanksby, S.J. J. Am. Soc. Mass Spectrom. 2010, 21, 1989-1999.

20. Preiss, J.; Loomis, C.R.; Bishop, W.R.; Stein, R.; Niedel, J.E.; Bell, R.M. J. Biol. Chem. 1986, 261, 8597-8600.

21. Billah, M.M.; Eckel, S.; Mullmann, T.J.; Egan, R.W.; Siegel, M.I. J. Biol. Chem. 1989, 264, 17069-17077.

22. Wang, M.; Hayakawa, J.; Yang, K.; Han, X. Anal. Chem. 2014, 86, 2146-2155.

23. Fhaner, C.J.; Liu, S.; Ji, H.; Simpson, R.J.; Reid, G. Anal. Chem. 2012, 84, 89178926.

24. Wang, M.; Kim, G.H.; Wei, F.; Chen, H.; Altarejos, J.; Han, X. Anal. Bioanal. Chem. 2015, 407, 5021-5032.

25. Wang, X.; Wei, F.; Xu, J.; Lv, X.; Dong, X.; Han, X.; Quek, S.; Huang, F.; Chen, H. Anal. Chim. Acta. 2016, 902, 142-153.

26. Wasslen, K.V.; Canez, C.R.; Lee, H.; Manthorpe, J.M.; Smith, J.C. Anal. Chem. 2014, 86, 9523-9532.

27. Canez, C.R.; Shields, S.W.J.; Bugno, M.; Wasslen, K.V.; Weinert, H.P.; Willmore, W.G.; Manthorpe, J.M.; Smith, J.C. Anal. Chem. 2016, 88, 6996-7004.

\subsection{Diazomethane synthesis}

Diazomethane (DZM) is a highly useful reagent for synthetic transformations due to its rapid reaction rate with acidic heteroatoms $\left(\mathrm{CO}_{2} \mathrm{H}, \mathrm{RNH}_{3}{ }^{+},(\mathrm{RO})_{2} \mathrm{P}(\mathrm{O}) \mathrm{OH}\right.$, etc. $)$ and

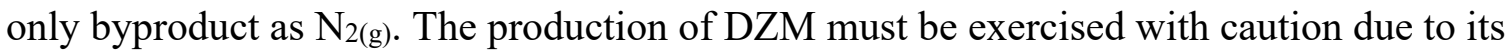
explosive and toxic nature, thus specialized procedures have been developed. In general, 
alkyl diazoalkanes are not stable for long periods of time and must be prepared from the reaction of $\mathrm{KOH}$ and suitable nitroso precursor. In our lab we have mastered the production of diazomethane from $N$-methyl- $N$-nitroso- $p$-toluenesulfonamide (Diazald ${ }^{\mathrm{TM}}$ ) and $N$ methyl- $N$-nitroso urea (NMU), where each have a different protocol for generating DZM. Figure 1.2.1 shows the experimental set-up for each precursor. Briefly, NMU is suspended in $\mathrm{Et}_{2} \mathrm{O}$ and cooled in an ice bath before a solution of $\mathrm{KOH}_{(\mathrm{aq})}$ is added with stirring. This method is the simplest and least expensive procedure of the two, however we have found that the DZM solution produced is contaminated with unreacted NMU and is not suitable for the reaction with biomolecules to be analyzed via MS. A cleaner alternative is the production of DZM from Diazald using a distillation method and specialized glassware. In this method an ethereal solution of Diazald is added dropwise to a heated mixture of EtOH, $\mathrm{H}_{2} \mathrm{O}$ and $\mathrm{KOH}$. Alkaline decomposition of Diazald produces gaseous DZM, in this state it is the most hazardous, can be co-distilled with $\mathrm{Et}_{2} \mathrm{O}$ and condensed on a cold finger with dry ice and isopropanol. 


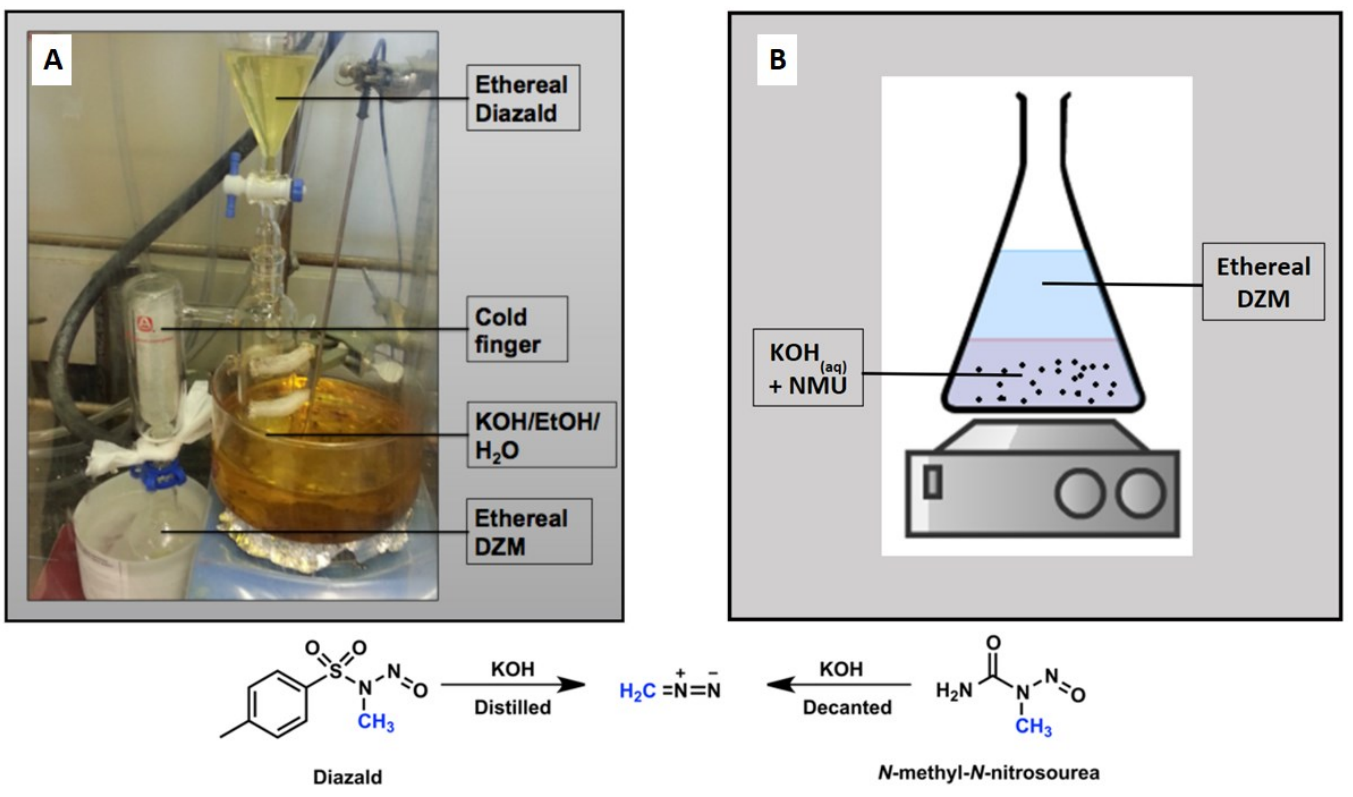

Figure 1.2.1 Two Methods of Preparing Ethereal Diazomethane From Diazald ${ }^{\mathrm{TM}}$ (A) and $N$-Methyl- $N$-Nitroso Urea (B) 


\section{Chapter 2: Trimethylation Enhancement using ${ }^{13} \mathrm{C}$-Diazomethane $\left({ }^{13} \mathrm{C}\right.$ - TrEnDi): Increased Sensitivity and Selectivity of Phosphatidylethanolamine, Phosphatidylcholine and Phosphatidylserine \\ Lipids Derived from Complex Biological Samples}

Carlos R. Canez, Samuel W. J. Shields, Magdalena Bugno, Karl V. Wasslen, Hillary P. Weinert, William G. Willmore, Jeffrey M. Manthorpe, and Jeffrey C. Smith. Anal. Chem. 2016, (88), 6996-7004

\subsection{Abstract}

Significant sensitivity enhancements in the tandem mass spectrometry-based analysis of complex mixtures of several phospholipid classes has been achieved via ${ }^{13} \mathrm{C}$ -

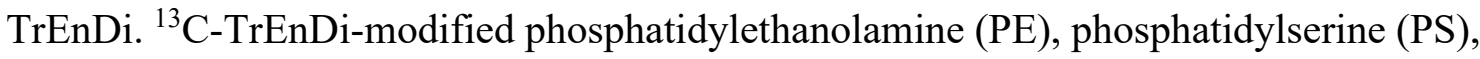
and phosphatidylcholine (PC) lipids extracted from HeLa cells demonstrated greater sensitivity via precursor ion scans (PIS) than their unmodified counterparts. Sphingomyelin (SM) species exhibited neither an increased nor decreased sensitivity following modification. The use of isotopically labeled diazomethane enabled the distinction of modified PE and modified PC species that would yield isobaric species with unlabeled diazomethane. ${ }^{13} \mathrm{C}$-TrEnDi created a PE-exclusive PIS of $\mathrm{m} / \mathrm{z}$ 202.1, two PSexclusive PISs of m/z 148.1 and m/z 261.1, and a PIS of m/z 199.1 for PC species (observed at odd $\mathrm{m} / \mathrm{z}$ values) and $\mathrm{SM}$ species (observed at even $\mathrm{m} / \mathrm{z}$ values). The standardized average area increase after TrEnDi modification was 10.72-fold for PE 
species, 2.36-fold for PC, and 1.05-fold for SM species. The sensitivity increase of PS species was not quantifiable, as there were no unmodified PS species identified prior to derivatization. ${ }^{13} \mathrm{C}$-TrEnDi allowed for the identification of $4 \mathrm{PE}$ and 7 PS species as well as the identification and quantitation of an additional 4 PE and 4 PS species that were below the limit of detection (LoD) prior to modification. ${ }^{13} \mathrm{C}-\mathrm{TrEnDi}$ also pushed $24 \mathrm{PE}$ and 6 PC lipids over the limit of quantitation (LoQ) that prior to modification were above the LoD only.

\subsection{Introduction}

Of the eight general lipid classes, ${ }^{1}$ glycerophospholipids constitute the majority of lipids in the cell and have been identified as key components in various cancers. ${ }^{2}$ Consequently, their study and identification as cellular biomarkers enables a greater understanding of disease and holds promise for new therapeutic avenues. ${ }^{3}$ Glycerophospholipids contain a glycerol backbone, a polar headgroup on the sn-3 position, and two fatty acyl groups with variable lengths and degrees of unsaturation on the sn-1 and sn-2 positions; ${ }^{2}$ the sn-3 group distinguishes between subclasses of glycerophospholipids. ${ }^{2}$ Sphingomyelin (SM), a sphingolipid, ${ }^{4}$ is a phosphocholine ester of a ceramide ${ }^{5}$ and hence is classified as a phospholipid.

Phosphatidylcholine (PC), phosphatidylethanolamine (PE), and phosphatidylserine (PS), three of the most common lipids in eukaryotic membranes, ${ }^{6}$ have been found to play important roles in cellular health and disease. ${ }^{3}$ For example, increases in intracellular levels of ether-linked PCs and PEs have been connected to metastatic colorectal cancer. ${ }^{2}$ Reduced levels of PC in the brain have been shown to lead to degeneration of cell membranes, which 
is directly linked to the development of Alzheimer's disease and allows PC to be used as a predictive disease biomarker. ${ }^{7}$ The production of $\mathrm{PC}$ in the brain is achieved by the methylation of PE, thereby linking both PC and PE to Alzheimer's disease. ${ }^{8}$ PE has also been identified as a eukaryotic membrane receptor for promoting antimicrobial activity through host defense peptides, a component of the immune system that works against a multitude of microbes as well as cancer cells. ${ }^{9}$ Sickle cells show increased expression of PS, contributing to a shortened erythrocyte lifespan and vascular occlusion, effectively escalating the disease state. ${ }^{10}$ Commonly found on the inner plasma membrane of cells, ${ }^{11}$ PS can also be used to identify tumor cells and hypoxic cardiomyocytes, which express PS on the surface of the cell, providing a method of identification. ${ }^{12}$ Atherosclerotic diseases, such as ischemic heart disease, have been linked to increased SM levels in the body, allowing SM to be used as a comprehensive biomarker. ${ }^{5}$ These and other similar discoveries have highlighted the importance of phospholipid characterization and analysis, leading to a significant increase in lipidomics research and literature. ${ }^{13}$ Most notably, mass spectrometry has been at the forefront of the revolutionary advancements in the field of lipidomics in recent years and is continually allowing insight into the composition of cellular membranes and identification of previously unknown bio-markers. ${ }^{3}$

Despite the prevalence of lipid research in recent years, there are still significant difficulties in this field. Many cellular biomarkers are present in lower concentrations, requiring enhanced sensitivity or novel methods of identification. Some improvements have been made by modifying the various species using isotopically labeled sulfonium ions, a fixed quaternary ammonium group, or amine group to impart a fixed positive charge. ${ }^{2,3,14}$ 
The methylation of ester fatty acids using diazomethane has enhanced their analysis via gas chromatography (GC) and MS. ${ }^{15-17}$ Dipalmitoylphosphatidylethanolamine has been successfully converted to dipalmitoylphosphatidyl [tris $\left.\left(\mathrm{N}-m e t h y l-{ }^{3} \mathrm{H}\right)\right]$ choline ${ }^{18}$ using diazomethane and $\mathrm{T}_{2} \mathrm{O}$ but was ineffective when attempting to concurrently methylate the primary amine and phosphate moieties of glycerophospholipids. The methylation of phosphate moieties in phosphoinositides has been achieved using trimethylsilyldiazomethane. ${ }^{19-21}$ The methylation of phosphate moieties and carboxylic acids in other phospholipid classes using trimethylsilyldiazomethane has been reported, but primary amines and other functional groups remain unmodified. ${ }^{22}$

We have previously developed novel, rapid, and cost-effective methods to enhance the sensitivity of phospholipid $\mathrm{MS}^{23}$ and proteomic MS analyses. ${ }^{24}$ Chemical derivatization of $\mathrm{PE}, \mathrm{PC}, \mathrm{SM}$, and PS via trimethylation enhancement using diazomethane ${ }^{23}$ led to the complete methylation of phosphate moieties, carboxylic acids, and primary amines, resulting in permanently positively charged analytes with a concomitant sensitivity enhancement. Sensitivity of tandem MS experiments was particularly improved, as ion fragmentation of derivatized analytes was consolidated to only one or two channels. ${ }^{23}$

In this study, we employ ${ }^{13} \mathrm{C}$-labeled diazomethane to address the issue of differentiation between isobaric PE and PC species created by derivatization with unlabeled diazomethane, a significant limitation of our initial studies. Furthermore, the effectiveness of ${ }^{13} \mathrm{C}-\mathrm{TrEnDi}$ in analyzing complex lipid extracts is demonstrated through the analysis of human cervical carcinoma (HeLa) cells. 


\subsection{Experimental}

Chemicals and Materials. Formic acid and tetrafluoroboric acid dimethyl ether complex were purchased from Sigma-Aldrich (St. Louis, MO, USA); absolute ethanol (EtOH) was purchased from Commercial Alcohols Inc. (Brampton, ON, Canada). Potassium hydroxide, methanol, chloroform, isopropanol, and ether were purchased from Caledon Laboratories Ltd. (Georgetown, ON, Canada); sodium acetate was purchased from Bioshop Canada Inc. (Burlington, ON, Canada). Sodium nitrite was obtained from BDH Chemicals Ltd. (Poole, England); glacial acetic acid, 28\% ammonium hydroxide, and ammonium acetate were obtained from Anachemia Canada Inc. (Montreal, QC, Canada). $\operatorname{PE}(16: 0 / 18: 1(9 Z)), \operatorname{PC}(16: 0 / 18: 1(9 Z)), \operatorname{PE}(8: 0 / 8: 0), \operatorname{PS}(8: 0 / 8: 0)$, and $\operatorname{PC}(5: 0 / 5: 0)$ were synthesized by and obtained from Avanti Polar Lipids Inc. (Alabaster, Alabama, USA). N(Methyl- ${ }^{13} \mathrm{C}$ )-N-nitroso-p-toluenesulfonamide was readily prepared from ${ }^{13} \mathrm{C}$-methanol (Cambridge Isotope Laboratories, Cambridge, Massachusetts, USA), an inexpensive and widely available ${ }^{13} \mathrm{C}$ label source, according to the procedure reported by Shields and Manthorpe. ${ }^{25}$

${ }^{13}$ C-Diazomethane Production. CAUTION: All reactions involving the preparation and use of diazomethane should be carried out in an efficient chemical fume hood and behind a safety blast shield because of the toxic and explosive nature of diazomethane. The production of ${ }^{13} \mathrm{C}$-diazomethane was performed analogously to unlabeled diazomethane using a Sigma-Aldrich Mini Diazald diazomethane generator with fire-polished clear-seal joints, as has been described elsewhere ${ }^{26}$ and previously reported by our groups. ${ }^{23-25}$ Cell Culture and Lipid Extraction. Human cervical carcinoma (HeLa) cells were purchased from ATCC (CCL-2) (Manassas, VA, USA) and grown using 87\% Eagle's minimum 
essential medium, $10 \%$ fetal bovine serum, and 3\% penicillin/streptomycin/antimycotic (Life Technologies Inc., Burlington, ON, Canada). Cells were cultured in a humidified atmosphere at $37{ }^{\circ} \mathrm{C}$ and $5 \% \mathrm{CO}_{2}$ using triple-gas incubators (Thermo Forma, Rockford, Illinois). Phospholipids were extracted using a modified Bligh and Dyer procedure. ${ }^{27}$ After extracting lipids in the chloroform phase, chloroform evaporation was followed by resuspension of the extracted lipidome in $600 \mu \mathrm{L}$ of EtOH.

In-Solution Chemical Derivatization. The same lipid extract was used for all modified and unmodified experiments. The lipid extract was divided into $25 \mu \mathrm{L}$ aliquots. In-solution chemical derivatization used $1.0 \mu \mathrm{L}$ of a freshly prepared and vigorously homogenized 14:1 (v/v) solution of diethyl ether and tetrafluoroboric acid dimethyl ether complex $\left(\mathrm{HBF}_{4} \cdot \mathrm{OMe}_{2}\right)$ after optimization experiments were performed. The rest of the in-solution chemical derivatization was performed as previously described by our group. ${ }^{23}$ Once completely dried, each aliquot of the modified lipidome extract was resuspended in $50 \mu \mathrm{L}$ of EtOH and $10 \mu \mathrm{L}$ of $8 \mu \mathrm{M} \mathrm{PC}(5: 0 / 5: 0)$ was added as a lipid standard to allow relative quantitation. As a control, aliquots of nonderivatized lipidome extract were dried down and resuspended in $50 \mu \mathrm{L}$ of EtOH and $10 \mu \mathrm{L}$ of $8 \mu \mathrm{M}$ PC (5:0/5:0) was added. Chemical derivatization was performed in triplicates.

Reversed Phase High Pressure Liquid Chromatography. Ten $\mu \mathrm{L}$ of the modified lipid sample containing the lipid standard was mixed with $30 \mu \mathrm{L}$ of deionized $\mathrm{H}_{2} \mathrm{O}$ and added to the autosampler of a Dionex Ultimate 3000 HPLC (Thermo Scientific, Odense, Denmark). Lipids were separated using a $\mathrm{C}_{4}$ reversed phase column and a ternary gradient program consisting of $30 \% \mathrm{MeOH}, 70 \% \mathrm{H}_{2} \mathrm{O}$ with $10 \mathrm{mM} \mathrm{NH}_{4} \mathrm{C}_{2} \mathrm{H}_{3} \mathrm{O}_{2}$ (mobile phase A), $100 \%$ IPA with $10 \mathrm{mM} \mathrm{NH}_{4} \mathrm{C}_{2} \mathrm{H}_{3} \mathrm{O}_{2}$ (mobile phase B), and $100 \%$ hexane (mobile phase 
C). The $\mathrm{C}_{4}$ column was prepared by packing a fritted $14 \mathrm{~cm}$ long piece of fused silica (200 $\mu \mathrm{m}$ ID) (Molex Inc., Lisle, IL, USA) with $5.5 \mathrm{~cm}$ of Reprosil-Pur $\mathrm{C}_{4}$ beads (Dr. Maisch GmbH HPLC, Ammerbuch-Entringen, Germany). The HPLC gradient began at 100\% A, increased to $100 \%$ B over 36.3 min, was followed by $10 \% \mathrm{~B} / 90 \% \mathrm{C}$ by $39.5 \mathrm{~min}$ at an average flow rate of $135 \mu \mathrm{L} / \mathrm{min}$. The run was then completed with a $2 \mathrm{~mL}$ system wash of $10 \% \mathrm{~B} / 90 \% \mathrm{C}$. Each analytical run was followed by a full blank run to further clean the system and ensure no lipid carryover occurred.

ESI-MS/MS Analyses. The HPLC eluent was introduced into an AB Sciex 4000 QTRAP via a Turbo V ionspray source (AB Sciex, Framingham, MA, USA). All LC-MS/MS studies were acquired in positive ion mode at an ESI voltage of $5000 \mathrm{~V}$, a scan rate of 250 Da per second, a declustering potential (DP) of $80 \mathrm{~V}$, and an ion source gas value (GS) of 20. For the modified and unmodified samples, five scans were performed per chromatographic run using low resolution for Q1 and unit resolution for Q3 (unless stated otherwise). The unmodified scans included three precursor ion scans (PISs) for $\mathrm{m} / \mathrm{z} 184.1$ from 400 to 450 Th (CE of $35 \mathrm{eV}$, lipid standard), 600 to 1300 Th (CE of $40 \mathrm{eV}, \mathrm{PC}$ species) and 600 to $900 \mathrm{Th}$ (CE of $40 \mathrm{eV}$ with Q1 and Q3 being set to unit resolution, PC and SM species), a NL scan for 141.1 Da from 600 to $1000 \mathrm{Th}$ (CE of $25 \mathrm{eV}$, PE species), and a NL scan for $185.1 \mathrm{Da}$ from 600 to $1000 \mathrm{Th}$ (CE of $23 \mathrm{eV}$, PS species). The modified scans included a PIS for m/z 184.1 from 400 to $450 \mathrm{Th}$ (CE of $35 \mathrm{eV}$, lipid standard), two PISs for m/z 199.1 from 615 to $1315 \mathrm{Th}$ (CE of $40 \mathrm{eV}, \mathrm{PC}^{\mathrm{Tr}}$ species) and 615 to $915 \mathrm{Th}$ (CE of $40 \mathrm{eV}$ with Q1 and Q3 being set to unit resolution, $\mathrm{PC}^{\mathrm{Tr}}$ and $\mathrm{SM}^{\mathrm{Tr}}$ species), a PIS for m/z 202.1 from 660 to $1060 \mathrm{Th}$ (CE of $40 \mathrm{eV}, \mathrm{PE}^{\mathrm{Tr}}$ species), and a PIS for m/z 148.1 from 675 to $1075 \mathrm{Th}$ (CE of $60 \mathrm{eV}, \mathrm{PS}^{\mathrm{Tr}}$ species). All samples were analyzed in triplicate. 
For the direct infusion analyses on PS, unmodified lipid extracts were resuspended in $75 \mu \mathrm{L}$ of EtOH with $10 \mathrm{mM} \mathrm{NH}_{4} \mathrm{C}_{2} \mathrm{H}_{3} \mathrm{O}_{2}$ or $75 \mu \mathrm{L}$ of EtOH with $10 \mathrm{mM} \mathrm{NH} \mathrm{H}_{4} \mathrm{OH}$ while modified samples were resuspended in $75 \mu \mathrm{L}$ of EtOH. The lipid extracts were directly infused into the ionspray source at a rate of $1.5 \mu \mathrm{L}$ per minute using a Harvard 11 Plus syringe pump (Harvard Apparatus, Holliston, MA, USA). An ESI voltage of $5000 \mathrm{~V}$ and DP of $40 \mathrm{~V}$ were used for positive ion mode scans and a voltage of $-4000 \mathrm{~V}$ and DP of $-40 \mathrm{~V}$ for negative ion scans. A scan rate of $250 \mathrm{Da} / \mathrm{s}$ and unit resolution for Q1 and Q3 were used for all scans. Final mass spectra were obtained by summing 30 scans. The unmodified scans included a positive NL for $185.1 \mathrm{Da}$ from 600 to $1000 \mathrm{Th}$ (CE of $23 \mathrm{eV}$ ) and a negative NL for $87.0 \mathrm{Da}$ from 600 to $1000 \mathrm{Th}(\mathrm{CE}$ of $-28 \mathrm{eV})$. The modified scans included a positive PIS for $\mathrm{m} / \mathrm{z} 148.1$ from 600 to 1000 Th $(\mathrm{CE}$ of $60 \mathrm{eV})$ and a positive PIS for m/z 261.1 from 600 to 1000 Th (CE of $40 \mathrm{eV}$ ). All samples were analyzed in triplicate.

\subsection{Results and Discussion}

We have recently reported the use of TrEnDi to create methylated derivatives of peptides $^{24}$ and several classes of lipids. ${ }^{23}$ TrEnDi has been demonstrated to rapidly and fully convert substrates/analytes into species that ionize with greater efficiency and fragment in a predictable and more sensitive manner. A key feature of this chemistry is the introduction of fixed, permanent positive charges via the formation of quaternary ammonium groups for PE and PS phospholipids. In the case of lipids, the use of $\mathrm{HBF}_{4}$ as a strong acid that forms a noncoordinating, non-nucleophilic conjugate base allows for the concomitant methylation of phosphate moieties, thereby permanently neutralizing their 
negative charge and affording PC, SM, PE, and PS phospholipids with a single permanent, fixed positive charge. Lipid derivatization via TrEnDi obviates the need for analyte protonation, eliminating ion suppression and rendering $\operatorname{TrEnDi}$ inherently quantitative. TrEnDi-derivatized glycerophospholipids do not form adducts (e.g., with sodium); the MS signal is consolidated into a single peak even in salty solvents, enhancing sensitivity. Furthermore, MS/ MS of TrEnDi-derivatized glycerophospholipids yields one or two fragmentation channels with high intensity, thus increasing sensitivity and simplifying data analysis. The TrEnDi chemistry is rapid and straightforward and uses a minimal amount of diazomethane; when carefully conducted behind a blast shield in a fumehood, the technique poses minimal risk to the user.

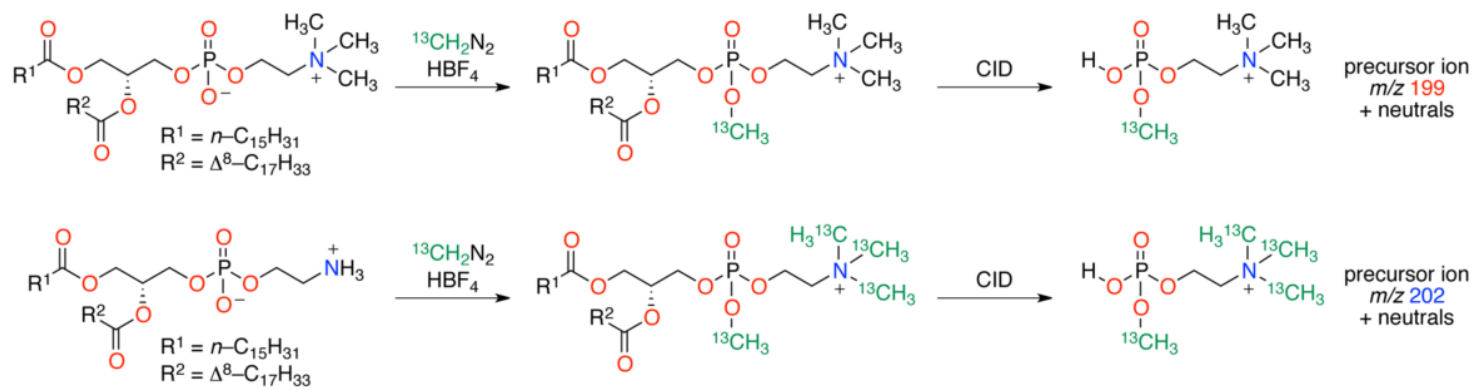

\section{Scheme 2.4.1 TrEnDi Derivatization of PC(16:0/18:1) and PE(16:0/18:1) with ${ }^{13} \mathrm{CH}_{2} \mathrm{~N}_{2}$}

Our initial publication on TrEnDi with lipids demonstrated significant sensitivity enhancements to PE analysis; however, the applicability of TrEnDi to biological samples was hampered by the fact that TrEnDi-modified PC derivatives $\left(\mathrm{PC}^{\mathrm{Tr}}\right)$ yield the same headgroup as TrEnDi-modified PE derivatives $\left(\mathrm{PE}^{\mathrm{Tr}}\right)$. This issue was overcome through the synthesis of ${ }^{13} \mathrm{C}$-labeled diazomethane ${ }^{25}$ for use in TrEnDi derivatization (Scheme 2.4.1). Derivatization with this reagent results in a PIS of $\mathrm{m} / \mathrm{z}$ 202.1, exclusive to $\mathrm{PE}$ species, and a PIS of m/z 199.1 for PC and SM species (Figure 2.4.1). 

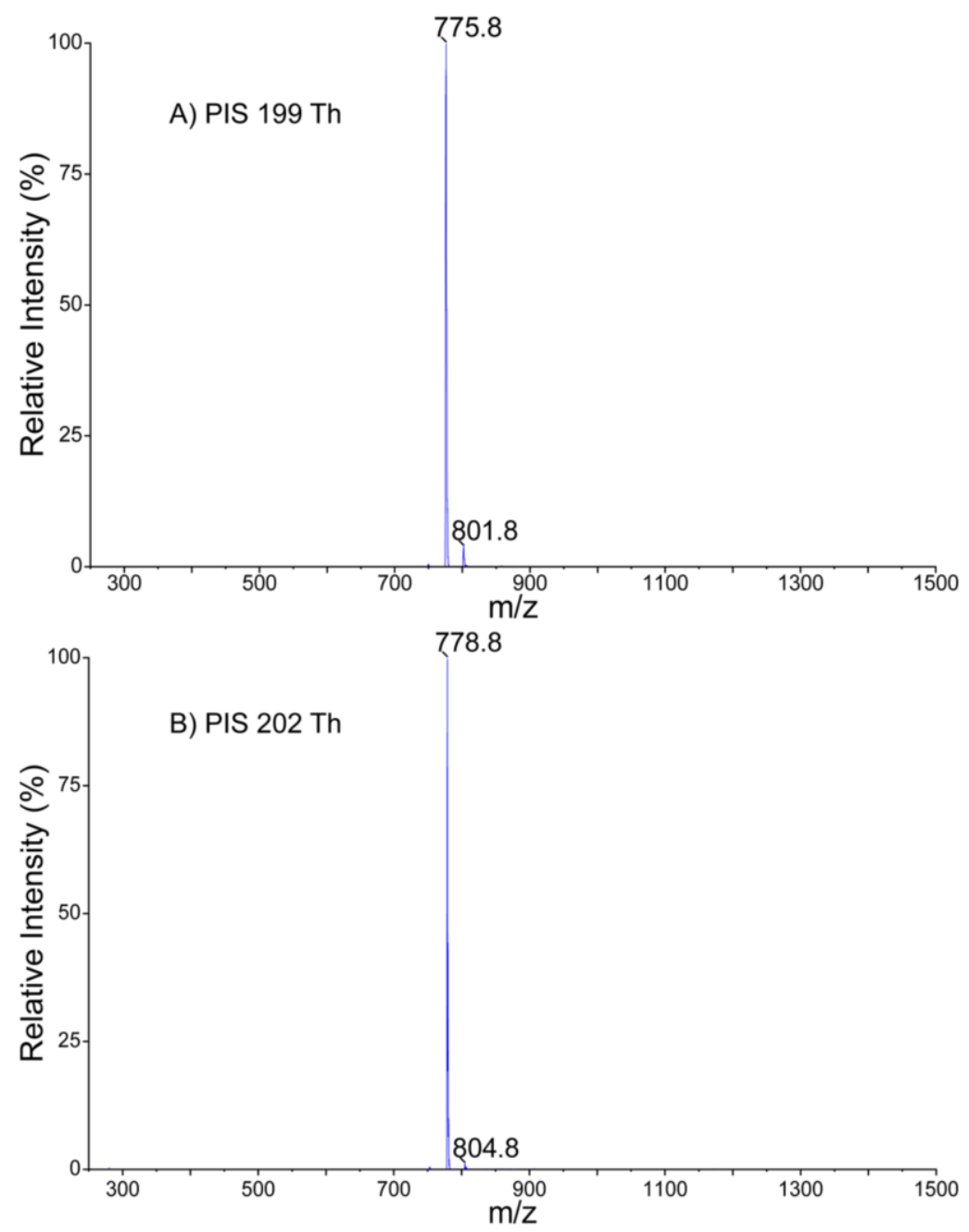

Figure 2.4.1 ${ }^{13} \mathrm{C}$-TrEnDi modification differentiates modified PE and PC species by unique PIS fragments of $\mathrm{m} / \mathrm{z} 199.1$ (A) and $\mathrm{m} / \mathrm{z} 202.1$ (B).

${ }^{13}$ C-TrEnDi on PE Analysis in HeLa Cells Lipid Extracts. As predicted, the sensitivity of PE analyses increased following ${ }^{13} \mathrm{C}$-TrEnDi modification and every PE increased in mass by $60 \mathrm{Da}$, as the three protons on the $1^{\circ}$ amine group and the proton on the phosphate moiety were each substituted with a ${ }^{13} \mathrm{C}$-labeled methyl group. The unmodified PE spectra were acquired via a NL scan of $141.1 \mathrm{Da}$, while the modified PE spectra were acquired via a PIS of $\mathrm{m} / \mathrm{z} 202.1$ (Figure 2.4.2). The three most intense 
unmodified PE peaks of 718.8, 744.8, and 768.6 Th in the unmodified spectrum (Figure 2A) correspond to the three tallest modified PE peaks of 778.9, 804.9, and 828.9 Th in the modified spectrum (Figure 2.4.2B). All modified PE peaks have a significantly stronger signal in comparison to their unmodified PE counterparts.
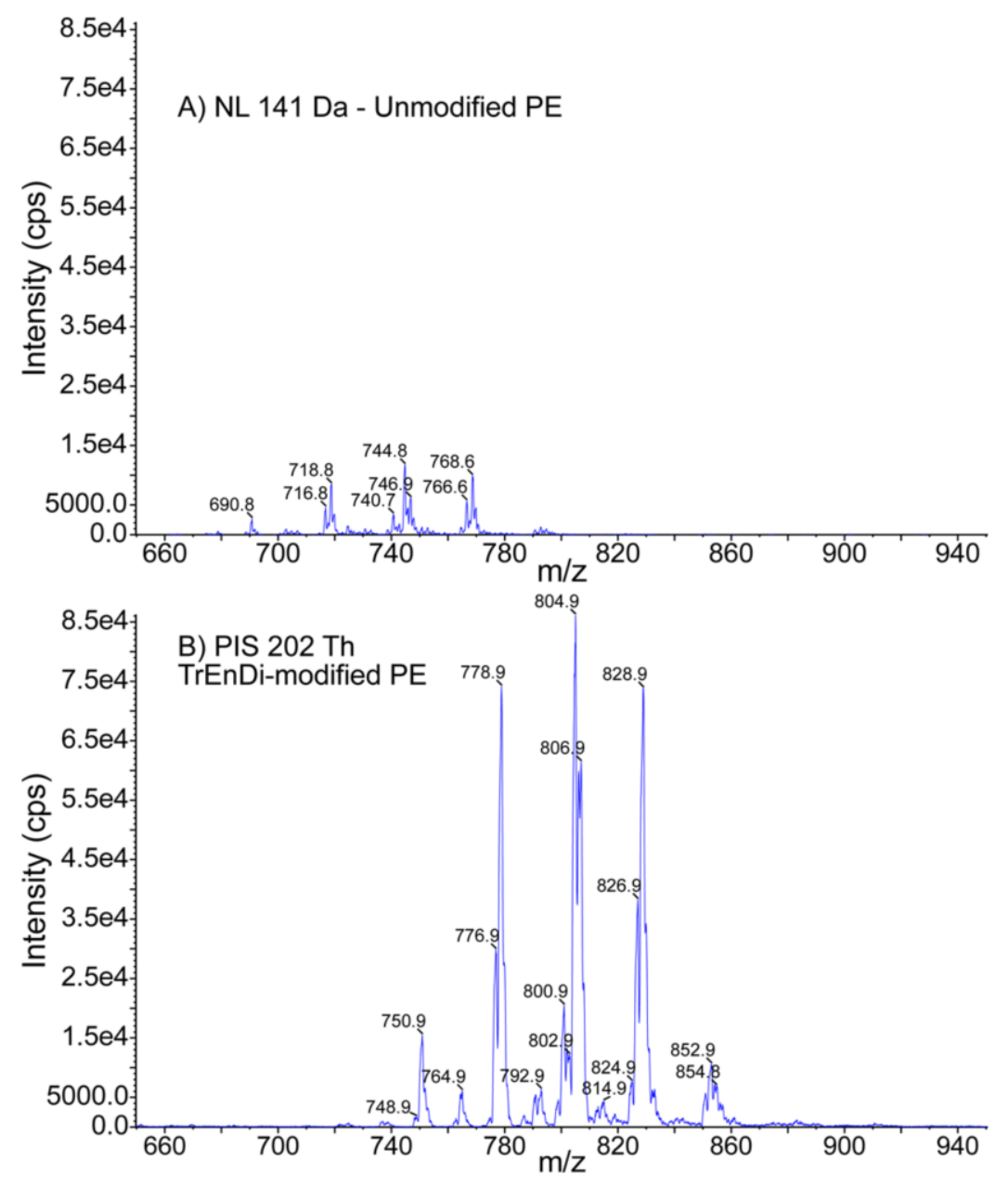

Figure 2.4.2 Mass Spectrum of unmodified PE from HeLa cell lipid extract acquired via +NL scan of 141.1 Da (A). Mass spectrum of ${ }^{13} \mathrm{C}$-TrEnDi-modified PE from HeLa extract acquired via +PIS of $\mathrm{m} / \mathrm{z} 202.1$ (B).

A total of 55 unmodified acyl and plasmanyl/O-alkyl PE species and 63 modified acyl and plasmanyl/O-alkyl PE species were identified, and all modified equivalents gained 
a higher intensity and area after derivatization. A comparison of the 20 highest standardized peak area averages between unmodified and modified PE triplicate runs are presented in Figure 3A. The TrEnDi-modified PE elution areas are higher following modification and show low standard deviations for both the unmodified and modified species. It is noteworthy that after TrEnDi modification we observed a significant sensitivity increase between peaks with area values that differed across 4 orders of magnitude, from the most abundant species (Figure 2.4.3) to the least-abundant species. All PE species experienced a statistically significant standardized area increase after TrEnDi modification.

Acid-based cleavage of vinyl ether moieties found in plasmalogens (a subclass of glycerophospholipids) has been well documented with a variety of different acids, ${ }^{28,}$ 29,2 including trifluoroacetic acid ${ }^{30,31}$ which has a $\mathrm{pK}_{\mathrm{a}}$ similar to $\mathrm{HBF}_{4} .{ }^{32}$ Our work presented here represents the first application of TrEnDi to a complex lipid extract from a biological sample and therefore the first time that our chemistry has been tested on plasmalogens. It was quickly discovered that the acidic conditions of TrEnDi do in fact cleave vinyl ether groups and therefore we removed the few instances of PE plasmalogen from the statistical analyses reported in this paper (7 PE plasmalogen species out of a total of 70 PE species; therefore, 63 acyl and O-alkyl PE species are reported here). In chapter 3 we investigate the ${ }^{13} \mathrm{C}-\mathrm{TrEnDi}$ modification of plasmalogen species in depth. 

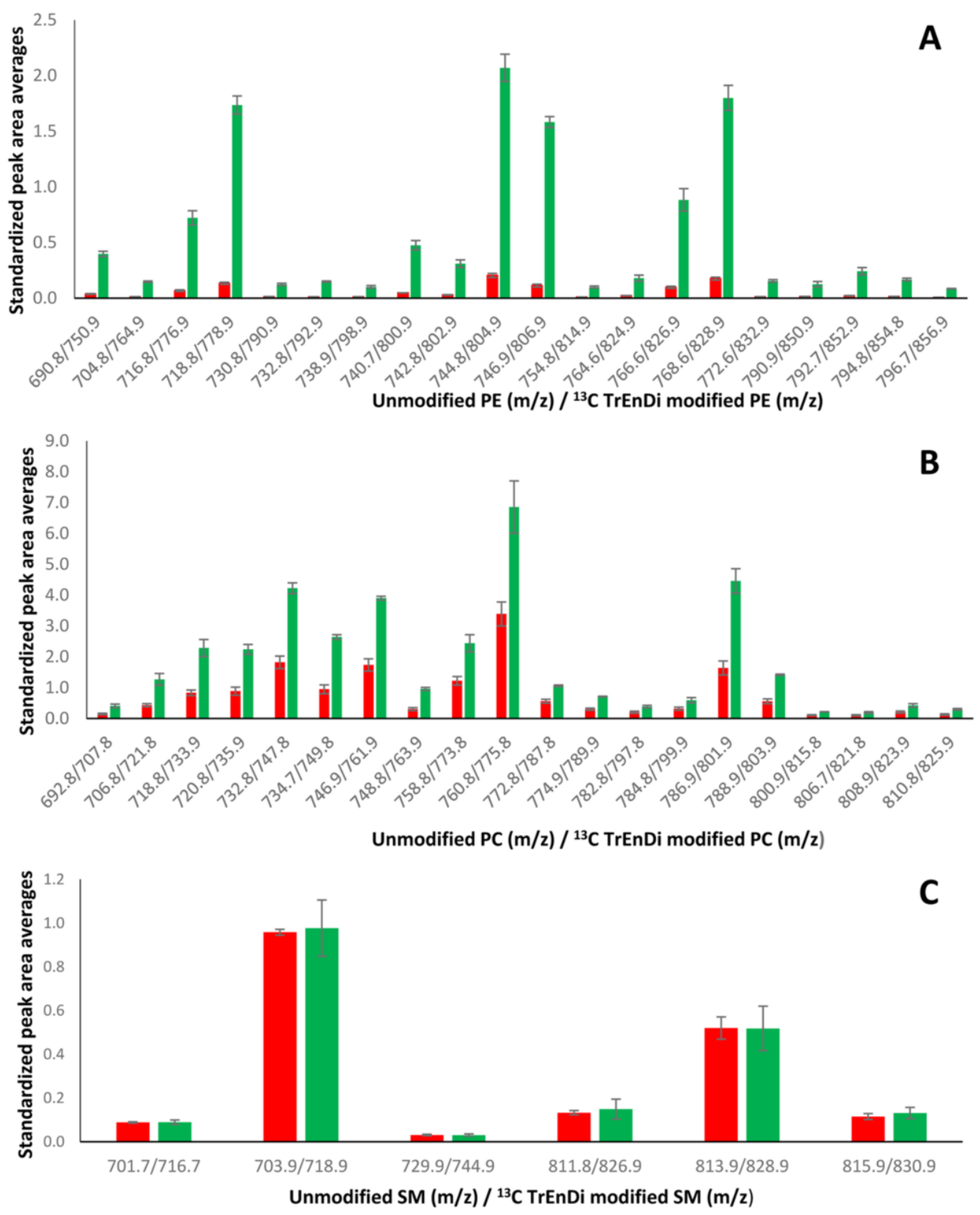

Figure 2.4.3 Comparison of standard peak area averages between unmodified triplicates (red bars) and ${ }^{13} \mathrm{C}$-TrEnDi chemical triplicates (green bars) for (A) PE, (B) PC, and (C) SM extracted from HeLa cells

An average standardized area fold increase of $10.72 \pm 2.01$ after ${ }^{13} \mathrm{C}$-TrEnDi modification (Table 2.4.1) was calculated by averaging all 30 quantifiable PE species. The 
small relative standard deviation values of PE standardized areas (Figures 2.4.3) calculated from ${ }^{13} \mathrm{C}$-TrEnDi chemical triplicates demonstrate a constant and reliable sensitivity increase. This is further corroborated by the small relative standard deviation average calculated from all $30 \mathrm{PE}$ species quantified (Table 2.4.1)

\section{Table 2.4.1 Summary of ${ }^{13} \mathrm{C}$-TrEnDi Enhancements on Identification and Quantitation of PE, PC, and SM in HeLa cells}

Quantitative Enhancements
$\begin{aligned} & \text { PE } \\ & \text { PC }\end{aligned}$

There were $24 \mathrm{PE}$ species whose area values increased from being above the limit of detection (LoD) threshold to being above the limit of quantitation (LoQ) threshold after modification with ${ }^{13} \mathrm{C}$-labeled diazomethane. This highlights the power of our technique to properly and accurately quantify lower abundant PE species that otherwise could only be detected. Four unmodified species with area values BLoD increased above the LoD threshold after TrEnDi modification and an additional four unmodified PE species with area values $\mathrm{BLoD}$ increased above the $\mathrm{LoQ}$ threshold. The area values of these four species 
lie slightly above their LoQ threshold, and when divided by 10.72, their calculated unmodified area values fall below their respective $\mathrm{LoD}$ threshold. ${ }^{13} \mathrm{C}-\mathrm{TrEnDi}$ was able to identify and quantify 8 low-abundance PE species that would otherwise be unidentifiable because they were below the noise level when using conventional PE analysis methods. The capability to identify and quantify low abundance lipids that may possess significant biological roles or be a strategic biomarker or molecular signaling molecule demonstrates the importance and potential of ${ }^{13} \mathrm{C}-\mathrm{TrEnDi}$ analysis.

${ }^{13} \mathrm{C}-\mathrm{TrEnDi}$ on PC and SM in HeLa Cells. PC and SM species experienced a $15 \mathrm{Da}$ mass increase following TrEnDi modification via ${ }^{13} \mathrm{C}$-methylation of the phosphate group; unmodified PC and SM spectra were acquired via PIS of $\mathrm{m} / \mathrm{z} 184.1$ while the modified PC and SM spectra were acquired via PIS of $\mathrm{m} / \mathrm{z}$ 199.1. PC species obtained a modest sensitivity increase following ${ }^{13} \mathrm{C}-\mathrm{TrEnDi}$ modification (see Figure S1). A total of 63 unmodified acyl and plasmanyl/O-alkyl PC species were identified prior to TrEnDi derivatization. All 63 were also observed after modification, and the 20 most intense signals are presented in Figure 2.4.3B. As with PE, PC species, despite having peak areas varying across 4 orders of magnitude, all displayed a sensitivity increase after TrEnDi (Figures 2.4.3). The overall small relative standard deviation values of PC standardized areas (Figures 2.4.3) calculated from ${ }^{13} \mathrm{C}$-TrEnDi chemical triplicates demonstrate a constant and reliable sensitivity enhancement. For the reasons described above in the case of PE, PC plasmalogens were removed from our data set (14 PC plasmalogens out of a total of 77 PC species; therefore, 63 acyl and plasmanyl/O-alkyl PC species are reported here). 
An average standardized area fold increase of $2.36 \pm 0.50$ after ${ }^{13} \mathrm{C}-\mathrm{TrEnDi}$ modification (Table 2.4.1) was calculated by averaging all 51 quantifiable PC species individual fold increase values. The $21.04 \%$ relative standard deviation average calculated from all 51 PC species quantified demonstrates a constant and reliable sensitivity increase across all PC species (Table 2.4.1). There were 6 PC species whose area values increased from above the LoD threshold to above the LoQ threshold after modification. A global sensitivity increase was achieved after ${ }^{13} \mathrm{C}-\mathrm{TrEnDi}$ modification, and several PC species that could only be identified before modification became quantifiable.

${ }^{13} \mathrm{C}$-TrEnDi modification did not lead to a sensitivity boost or hindrance for SM species. A total of 6 SM species were identified in the unmodified and modified spectra and are presented in Figure 2.4.3C. After performing average standardized area fold increase calculations for the six SM species, a value of $1.05 \pm 0.07$ was obtained (Table 2.4.1). Analysis of the PC (5:0/5:0) external standard on its own revealed a SM contaminant with an $\mathrm{m} / \mathrm{z}$ of 675.8 . The lipid standard solution was always added after TrEnDi modification, and the fold increase for the modified $690.8 \mathrm{~m} / \mathrm{z}$ peak demonstrates a 0.53 fold increase, revealing the amount of indigenous $675.8 \mathrm{~m} / \mathrm{z} \mathrm{SM}$ in HeLa cells happened to be approximately the same amount as the standard's SM contaminant. As presented in our previous work, ${ }^{23}$ the hydroxyl moiety of the ceramide component of SM species is partially methylated following TrEnDi derivatization and results in signal splitting for SM species. This explains why there is no overall sensitivity enhancement for SM despite both PC and SM species possessing a phosphocholine headgroup. Future work in our groups will seek to address this issue. 
${ }^{13} \mathrm{C}$-TrEnDi Methylation Efficiency. In order to ensure quantitative performance of ${ }^{13} \mathrm{C}-\mathrm{TrEnDi}$, the percent yield (methylation efficiency) of the chemical derivatization for PE, PC, and SM species present in the HeLa cell lipid extract was assessed by scanning for unmodified species in the modified aliquots using optimal unmodified conditions (including a protonation source). Since PC and SM exhibit a single ${ }^{13} \mathrm{C}$-methyl addition, analyzing their unmodified counterparts in the derivatized lipid solution directly provides the percent yield of the chemical derivatization.

In order to quantify the percentage of unmodified lipids after ${ }^{13} \mathrm{C}-\mathrm{TrEnDi}$ modification, unmodified species with area values higher than the LoQ threshold were used. An average of $0.37 \%$ standardized area was observed for unmodified PC after TrEnDi modification, thus demonstrating a virtually quantitative yield of $99.63 \%$. For SM and PE species, a $100 \%$ yield was obtained since there were no unmodified remaining species with areas higher than the LoQ threshold. Even when using unmodified area values above the LoD (and LoQ) threshold, an average of $0.30 \%$ unmodified PC standardized area remained, while using all unmodified area values (BLoD, LoD, and LoQ) revealed 1.08\% unmodified PC standardized area remained, a $0.42 \%$ unmodified SM standardized area remained, and $0.55 \%$ unmodified PE standardized area remained.

In order to properly assess the percent yield for PE species, PIS and NL scans corresponding to full methylation and lack of $1,2,3$, or $4{ }^{13} \mathrm{C}$-methylation events were performed on three of the highest intensity PE species found in the HeLa cell extract (fully modified $\mathrm{m} / \mathrm{z}$ values of $804.9,806.9$, and 828.9 ). The methylation efficiency results showed no remaining unmodified areas above the LoD threshold for all the PIS and NL scans. This confirmed a $100 \%$ yield of fully modified PE post ${ }^{13} \mathrm{C}$-TrEnDi modification. 
The content of ${ }^{12} \mathrm{C}$ and ${ }^{13} \mathrm{C}$ after synthesis of ${ }^{13} \mathrm{C}$-diazomethane from ${ }^{13} \mathrm{C}$ methanol $^{25}$ was evaluated with MS quantitation experiments. The synthesis yielded $99.07 \%{ }^{13} \mathrm{C}$-diazomethane and $0.93 \%{ }^{12} \mathrm{C}$-diazomethane.

${ }^{13} \mathrm{C}-\mathrm{TrEnDi}$ on PS in HeLa Cells. All PS species exhibited a mass shift of $75 \mathrm{Da}$ following ${ }^{13} \mathrm{C}$-TrEnDi modification, corresponding to the exchange of five protons for five ${ }^{13} \mathrm{C}$-labeled methyl groups in the carboxylic acid, amine $(\times 3)$, and phosphate groups of the PS headgroup. ${ }^{13} \mathrm{C}$-TrEnDi modification provided an outstanding sensitivity increase to PS species. There were no unmodified species above the LoD identified by the +NL scan of 185.1 Da or the $-\mathrm{NL}$ scan of $87.0 \mathrm{Da}$ (Figure 2.4.4A,B). In contrast, the modified scan of + PIS of $\mathrm{m} / \mathrm{z} 261.1$ (Figure 4D) was able to identify three PS species above the LoQ and one above the LoD, while the +PIS of $\mathrm{m} / \mathrm{z} 148.1$ (Figure 4C) was able to identify three PS species above the LoQ and seven above the LoD. Both modified scans identified the same PS species and highlight the importance of our technique in identifying and quantifying phospholipid species that otherwise would go unidentified. It is important to note that not all of the peaks labeled in Figure 2.4.4 have corresponding area values above the LoD; some peaks have intensities greater than the noise level but have area values below the LoD (e.g., m/z 809.8, 823.8, 887.7, 891.9, and 913.7 in Figure 4D). Conversely, m/z 835.9, 889.9, and 913.9 (Figure 2.4.4C) had area values above the LoD but low intensity values. It was therefore determined that, at the mass resolution used in this work, area values are significantly more reliable than intensity values, and only area LoD and area LoQ thresholds were calculated. 

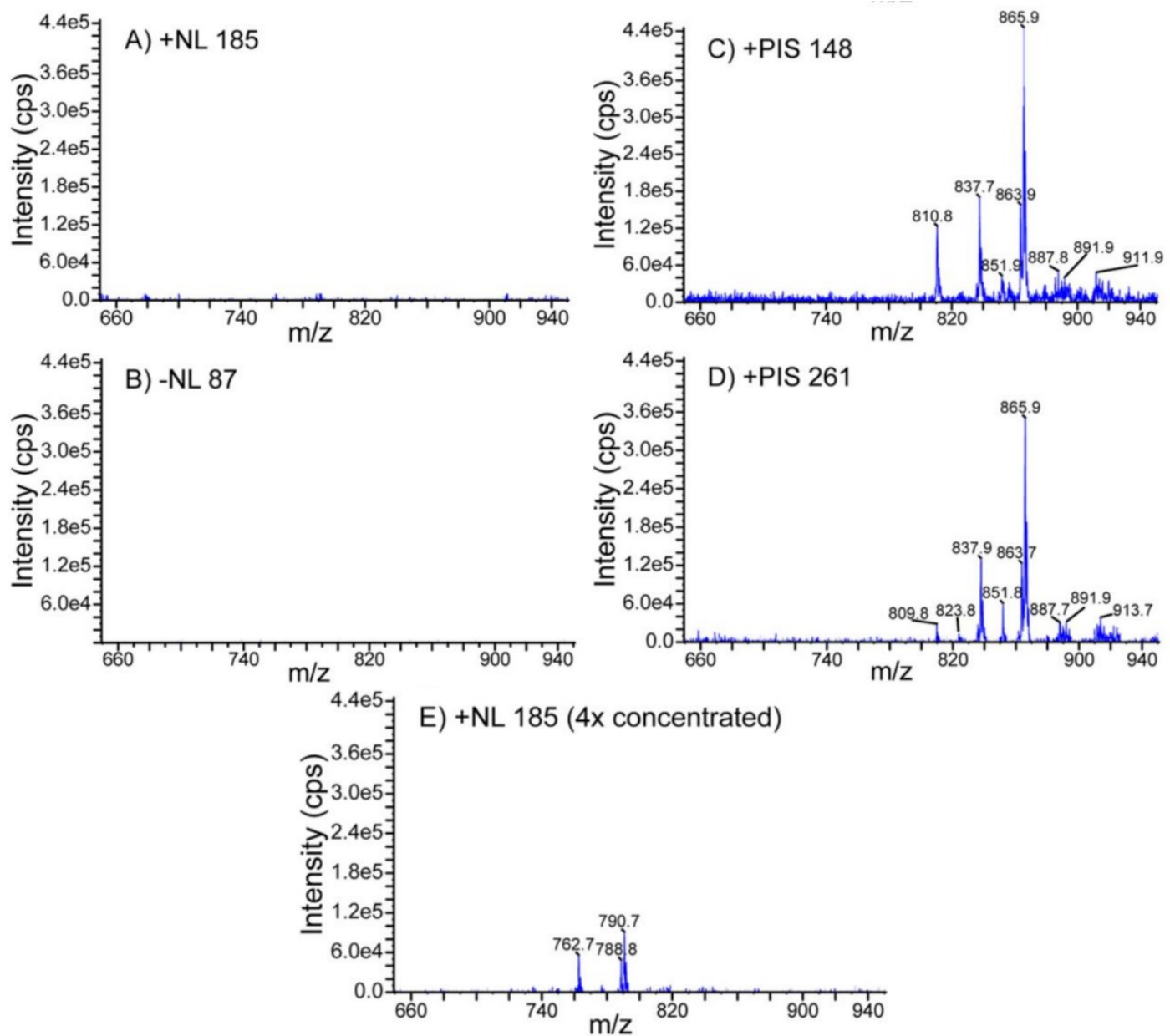

Figure 2.4.4 Unmodified HeLa cell lipodome was dissolved in EtOH with $10 \mathrm{mM}$ ammonium acetate or $10 \mathrm{mM}$ ammonium hydroxide and analyzed via their respective +NL of 185 Da (A) or -NL of 87 Da scandal (B). The ${ }^{13}$ C-TrEnDimodified extracts were analyzed via +PIS of $\mathrm{m} / \mathrm{z} 148.1$ (C) and +PIS of $\mathrm{m} / \mathrm{z} 261.1$ (D). Unmodified 4x concentrated PS was analyzed using +NL 185.1 Da (E)

Unlike the PE, PC, and SM experiments that were performed via LC-MS, the PS studies were performed via direct infusion after realization that modified PS species were not compatible with the solvents used in our HPLC separation. In contrast, $100 \% \mathrm{EtOH}$ direct infusion experiments provided no hindrance to the modified PS spectrum, 
demonstrating its efficacy for direct spray studies. A TrEnDi modified PS (8:0/8:0) standard was successfully analyzed in $100 \% \mathrm{EtOH}$ via direct spray but also demonstrated lack of compatibility with our LC-MS separation solvents, indicating this was not a samplespecific occurrence.

In order to determine that the peaks observed in the PIS of m/z 261.1 and 148.1 were modified PS species, the unmodified aliquot was concentrated by a factor of 4 and analyzed via a +NL scan of 185.1 Da. This revealed three peaks above the LoD with $\mathrm{m} / \mathrm{z}$ values of 762.7, 788.8, and 790.7 (Figure 2.4.4E). These three peaks correspond with the three tallest peaks identified by both modified scans, demonstrating that the three peaks are indeed modified PS species whose areas increased above the LoQ threshold from noise after modification. Even after a 4-fold concentration of the unmodified sample, the average area values are significantly lower and there are fewer PS species identified in comparison to the modified scans (Figure 2.4.4). Having two different PISs (m/z 261.1 and 148.1) for TrEnDi-modified PS species enabled the identification of a non-PS contaminant at $\mathrm{m} / \mathrm{z} 810$ via its exclusivity to the PIS of $\mathrm{m} / \mathrm{z}$ 148.1. This approach also provided confident identification of the other peaks as modified PS species, since only modified PS head groups would be able to dissociate via both fragmentation pathways. The $\mathrm{m} / \mathrm{z} 810$ contaminant peak has an even $\mathrm{m} / \mathrm{z}$ value, while all ${ }^{13} \mathrm{C}-\mathrm{TrEnDi}$ modified PS species have odd masses, and is absent in the PIS of $\mathrm{m} / \mathrm{z} 261.1$, thus indicating that it is not a PS.

The modified PIS of $\mathrm{m} / \mathrm{z} 148.1$ is $1.49 \pm 0.02$ times more sensitive than the PIS of $\mathrm{m} / \mathrm{z} 261$ but has a higher noise background as a result of using a higher CE and showed a non-PS contaminant in the spectrum (Figure 2.4.4C). The PIS of m/z 261 has a lower background noise and is exclusive for modified PS peaks making it the better scan for 
identification and quantitation (Figure 2.4.4D). Having two different scans easily verifies the polar headgroup identity of PS species. Even though the background noise in both scans for the TrEnDi-modified PS species is higher than that for unmodified PS species, leading to a mildly higher $\mathrm{LoD}$ and $\mathrm{LoQ}$, the sensitivity boost obtained post ${ }^{13} \mathrm{C}-\mathrm{TrEnDi}$ modification is greater than an order of magnitude and provides the ability to identify and quantify PS species that otherwise would be invisible via conventional methods.

\subsection{Conclusion}

The creation of permanently charged lipid analytes that do not require protonation and are relatively immune to ion suppression make ${ }^{13} \mathrm{C}-\mathrm{TrEnDi}$ an inherently quantitative technique with enhanced sensitivity compared to conventional analysis methods. Permanently charged analytes will provide the same signal regardless of the complexity of the sample being analyzed. ${ }^{13} \mathrm{C}-\mathrm{TrEnDi}$ modification boasts percent yield values higher than $99.63 \%$ for complete methylation in all four phospholipid classes; the synthesis of ${ }^{13} \mathrm{C}$-diazomethane from ${ }^{13} \mathrm{C}$-methanol affords material with a $99.07 \%{ }^{13} \mathrm{C}$ content, corroborating the quantitative nature of the technique. Even after thorough optimization of unmodified phospholipid studies to achieve maximum sensitivity via conventional MS/MS methods, ${ }^{13} \mathrm{C}$-TrEnDi conclusively demonstrated significant sensitivity enhancements for phospholipids. In the case of PE and PS species, ${ }^{13} \mathrm{C}-\mathrm{TrEnDi}$ enabled the identification and quantitation of several species that were below the limit of quantitation and detection prior to modification. A modest increase for PC species enabled the possibility of quantitation of several PC species. Modification of the sample does not hinder the identification or quantitation of SM species, enabling their study in modified samples just as well as their 
unmodified equivalents. ${ }^{13} \mathrm{C}$-TrEnDi on biological lipid extracts proved to be beneficial, easy, rapid, cost-effective, and compatible with LCMS and direct infusion experiments, with the exception of PS studies, which remain exclusive to direct infusion studies. ${ }^{13} \mathrm{C}$ TrEnDi is amenable to PE, PS, and PC lipids and is a powerful tool to identify and quantify low abundance phospholipids in biological systems. ${ }^{13} \mathrm{C}-\mathrm{TrEnDi}$ holds the promise to be a key tool to discover novel lipid components in cellular signaling pathways or biomarkers of a particular diseased state, which otherwise would go undetected via conventional methods.

\subsection{References}

1. Fahy, E.; Subramaniam, S.; Murphy, R. C.; Nishijima, M.; Raetz, C. R.; Shimizu, T.; Spener, F.; van Meer, G.; Wakelam, M. J.; Dennis, E. A. J. Lipid Res. 2009, 50 (Suppl), S9-S14.

2. Fhaner, C. J.; Liu, S.; Ji, H.; Simpson, R. J.; Reid, G. E. Anal. Chem. 2012, 84, 8917-8926.

3. Wang, M.; Hayakawa, J.; Yang, K.; Han, X. Anal. Chem. 2014, 86, 2146-2155.

4. Bou Khalil, M.; Hou, W.; Zhou, H.; Elisma, F.; Swayne, L. A.; Blanchard, A. P.; Yao, Z.; Bennett, S. A. L.; Figeys, D. Mass Spectrom. Rev. 2010, 29, 877-929.

5. Ohkawa, R.; Kishimoto, T.; Kurano, M.; Dohi, T.; Miyauchi, K.; Daida, H.; Nagasaki, M.; Uno, K.; Hayashi, N.; Sakai, N.; Matsuyama, N.; Nojiri, T.; Nakamura, K.; Okubo, S.; Yokota, H.; Ikeda, H.; Yatomi, Y. Clin. Biochem. 2012, $45,1463-1470$. 
6. van Meer, G.; Voelker, D. R.; Feigenson, G. W. Nat. Rev. Mol. Cell Biol. 2008, 9, $112-124$

7. Whiley, L.; Sen, A.; Heaton, J.; Proitsi, P.; García-Gomez, D.; Leung, R.; Smith, N.; Thambisetty, M.; Kloszewska, I.; Mecocci, P.; Soininen, H.; Tsolaki, M.; Vellas, B.; Lovestone, S.; Legido-Quigley, C. Neurobiol. Aging 2014, 35, 271-278.

8. Guan, Z. Z.; Wang, Y. N.; Xiao, K. Q.; Hu, P. S.; Liu, J. L. Neurochem. Int. 1999, $34,41-47$.

9. Phoenix, D. A.; Harris, F.; Mura, M.; Dennison, S. R. Prog. Lipid Res. 2015, 59, $26-37$.

10. van Tits, L. J.; van Heerde, W. L.; Landburg, P. P.; Boderie, M. J.; Muskiet, F. A. J.; Jacobs, N.; Duits, A. J.; Schnog, J. B. Biochem. Biophys. Res. Commun. 2009, $390,161-164$.

11. Bevers, E. M.; Comfurius, P.; Dekkers, D. W.; Harmsma, M.; Zwaal, R. F. Biol. Chem. 1998, 379, 973-986.

12. Schutters, K.; Reutelingsperger, C. Apoptosis 2010, 15, 1072-1082.

13. Brugger, B. Annu. Rev. Biochem. 2014, 83, 79-98.

14. Distler, U.; Hülsewig, M.; Souady, J.; Dreisewerd, K.; Haier, J.; Senninger, N.; Friedrich, A. W.; Karch, H.; Hillenkamp, F.; Berkenkamp, S.; Peter-Katalinic,J.; Müthing, J. Anal. Chem. 2008, 80, 1835-1846.

15. Graff, G.; Anderson, L. A.; Jaques, L. W.; Scannell, R. T. Chem. Phys. Lipids 1990, $53,27-36$.

16. Mueller, H. W. J. Chromatogr., Biomed. Appl. 1996, 679, 208-209.

17. Schlenk, H.; Gellerman, J. L. Anal. Chem. 1960, 32, 1412-1414. 
18. Smith, G. A.; Montecucco, C.; Bennett, J. P. Lipids 1978, 13, 92-94.

19. Clark, J.; Anderson, K. E.; Juvin, V.; Smith, T. S.; Karpe, F.; Wakelam, M. J. O.; Stephens, L. R.; Hawkins, P. T. Nat. Methods 2011, 8, 267-272.

20. Kielkowska, A.; Niewczas, I.; Anderson, K. E.; Durrant, T. N.; Clark, J.; Stephens, L. R.; Hawkins, P. T. Adv. Biol. Regul. 2014, 54, 131-141.

21. Cai, T.; Shu, Q.; Hou, J.; Liu, P.; Niu, L.; Guo, X.; Liu, C. C.; Yang, F. Anal. Chem. 2015, 87, 513-521.

22. Lee, J. W.; Nishiumi, S.; Yoshida, M.; Fukusaki, E.; Bamba, T. J. Chromatogr. A 2013, 1279, 98-107.

23. Wasslen, K. V.; Canez, C. R.; Lee, H.; Manthorpe, J. M.; Smith, J. C. Anal. Chem. 2014, 86, 9523-9532.

24. Wasslen, K. V.; Tan, L. H.; Manthorpe, J. M.; Smith, J. C. Anal. Chem. 2014, 86, $3291-3299$.

25. Shields, S. W. J.; Manthorpe, J. M. J. Labelled Compd. Radiopharm. 2014, 57, $674-679$.

26. Aldrich Technical Bulletin AL-180 [Online]; Sigma-Aldrich: St. Louis, MO, 2007.

27. Bonin, F.; Ryan, S. D.; Migahed, L.; Mo, F.; Lallier, J.; Franks, D. J.; Arai, H.; Bennett, S. A. J. Biol. Chem. 2004, 279, 52425-52436.

28. Murphy, E. J.; Stephens, R.; Jurkowitz-Alexander, M.; Horrocks, L. A. Lipids 1993, $28,565-568$.

29. Leßig, J.; Schiller, J.; Arnhold, J.; Fuchs, B. J. Lipid Res. 2007, 48, 1316-1324.

30. Nimptsch, A.; Fuchs, B.; Süß, R.; Zschörnig, K.; Jakop, U.; Göritz, F.; Schiller, J.; Müller, K. Anal. Bioanal. Chem. 2013, 405, 6675-6682. 
31. Haynes, W. M., Ed. TFA (pKa 0.52): Dissociation Constants of Organic Acids and Bases. In CRC Handbook of Chemistry and Physics, $96^{\text {th }}$ Edition (Internet Version 2016) [Online]; CRC Press/Taylor and Francis: Boca Raton, FL, 2016; Chapter 5, pp 94.

32. Haynes, W. M., Ed. HBF4 (pKa 0.5): Dissociation Constants of Inorganic Acids and Bases. In CRC Handbook of Chemistry and Physics, 96 ${ }^{\text {th }}$ Edition (Internet Version 2016) [Online]; CRC Press/Taylor and Francis: Boca Raton, FL, 2016; Chapter 5, pp 93. 


\title{
Chapter 3: Detection of Plasmenyl-Ether Glycerophospholipids in Bovine Liver Extract via a Dual Chemical Derivatization Strategy
}

\author{
Samuel W. J. Shields ${ }^{\mathrm{a}, \mathrm{c}}$, Carlos R. Canez ${ }^{\mathrm{b}, \mathrm{c}}$, Peter J. Pallister ${ }^{\mathrm{a}, \mathrm{c}}$, Jeffrey M. Manthorpe ${ }^{\mathrm{a}, \mathrm{b}, \mathrm{c} *}$, \\ Jeffrey C. Smith ${ }^{\mathrm{a}, \mathrm{b}, \mathrm{c} *}$ \\ a) Department of Chemistry Carleton University, Ottawa, Ontario K1S 5B6, \\ Canada \\ b) Institute of Biochemistry, Carleton University, Ottawa, Ontario K1S 5B6, \\ Canada \\ c) Carleton Mass Spectrometry Centre, Carleton University, Ottawa, Ontario \\ K1S 5B6, Canada \\ * to whom correspondence should be addressed - jeff.smith@carleton.ca, \\ jeffrey.manthorpe@,carleton.ca
}

Keywords: lipids, glycerophospholipids, diazomethane, plasmalogens, mass spectrometry

\subsection{Abstract}

Plasmalogen identification and quantitation can be challenging due to their sensitivity to acid degradation, isobaric overlap with plasmanyl ether-linked glycerophospholipids and low abundance further complicates analysis. The TrEnDi protocol can be used to significantly enhance the signal of glycerophospholipids by the creation of a fixed positive charge via methylation using ${ }^{13} \mathrm{C}-\mathrm{DZM}$ in complex lipid extract. Although TrEnDi requires a strong acid for complete methylation, optimization of the protocol to use $10 \mathrm{mM} \mathrm{HBF}_{4}$ during the reaction and the subsequent addition of a buffer 
solution post diazomethane addition, prevents the acidic hydrolysis of plasmalogens enabling their analysis via TrEnDi. These optimized conditions were applied to aliquots of bovine liver extract (BLE) to achieve full methylation of plasmalogen lipids within a complex mixture. Twenty-nine plasmalogen phospholipids were identified by treating a portion of underivatized and TrEnDi BLE samples with an $80 \%$ formic acid and comparing the LCMS results to the untreated samples. On average, methylated plasmalogens from BLE were found to be 2.81- and 28.1-fold for PC and PE plasmalogens, respectively. Furthermore, the dual derivatization of plasmalogens with ${ }^{13} \mathrm{C}-\mathrm{TrEnDi}$ and a previously reported iodoactalization strategy has been employed to effectively identify plasmanylether lipids in complex biological extracts. In summary, optimized TrEnDi modification with ${ }^{13} \mathrm{C}$-DZM was used to encompass the analysis of plasmalogen lipids with no undesired cleavage of radyl groups and boost their signal in LCMS analyses.

\subsection{Introduction}

The structural diversity of glycerophospholipids (GPL) is remarkable, having several variations on the phosphate headgroup (ethanoloamine, choline, serine, glycerol, etc.) as well as a plethora of different possible radyl groups on the glycerol backbone (chain length, level and location of unsaturation, and type of linkage to the backbone). This structural diversity is an attribute of GPLs that allows all biological systems to function but makes their characterization by analytical mass spectrometry challenging. Lipids with ether-linked components are a unique subclass of glycerophospholipids (GPL) that contain a hydrocarbon chain connection as an ether at the $s n-1$ position as either an 1-O-alkyl ether (plasmanyl) or 1-O-alkenyl ether (plasmenyl). Plasmenyl phospholipids, also referred to as 
plasmalogens, represent $20 \%$ of all phospholipid mass in humans and play central roles in many key areas such as membrane fusion, protection against oxidative degradation, and regulation of membrane-bound proteins. ${ }^{1}$ The major components ether linked GPL species found in nature are ethanolamine plasmalogen (PlsEtn) and choline plasmalogen (PlsCho) which bear the phosphoethanolamine (PE) and phosphocholine (PC) head groups at $s n-3$ respectively.

Although the PlsEtn subclass predominates over the PlsCho in abundance, these species have been found in human heart, brain, liver, and blood plasma in varying amounts. ${ }^{2}$ Plasmalogen levels have been shown to be found in greater quantities in the biofluids and tissues of various cancer types, in particular, gastrointestinal and pancreatic cancer where they have been correlated to oncogenic signalling. ${ }^{3}$ Patients with Alzheimer's disease have been shown to have decreased levels of plasmalogens compared to control subjects, where the most significant decrease in PlsEtn could be found in the hippocampus and frontal cortex. ${ }^{4}$ Recently, a study used PlsEtn as a therapeutic agent for the treatment of memory loss in amyloid $\beta$-infused rats. ${ }^{5}$ The development of streamlined techniques for the analysis of plasmalogens that can reliably facilitate the identification and quantitation of these biologically important lipid species will further our understanding of their involvement in various diseases, as well as open avenues for accelerated diagnosis and may facilitate the development of new treatments.

Analysis using mass spectrometry is at the forefront for reliable identification and quantitation of lipids in complex biological samples. ${ }^{6}$ In the case of plasmenyl ether GPLs, mass spectrometry (MS) has been the leading technology for their analysis, albeit with the caveat of requiring additional techniques such as chemical derivatization and/or tandem 
MS $\left(\mathrm{MS}^{2}\right)$ for complete characterization. The challenge of a study focused on the identification of plasmalogens is two-fold. Firstly there is a large amount of isobaric interference with other structurally similar lipid species (e.g. $[\mathrm{PE}(\mathrm{P}-36: 4)+\mathrm{H}]^{+}=[\mathrm{PC}(\mathrm{P}-$ $\left.33: 4)+\mathrm{H}]^{+}=[\mathrm{PE}(\mathrm{O}-36: 5)+\mathrm{H}]^{+}=[\mathrm{PC}(\mathrm{O}-33: 5)+\mathrm{H}]^{+}\right)$. Secondly identification via $\mathrm{MS}^{2}$ can produce fragments unique to plasmalogen species ${ }^{7,8}$ but can be easily overlooked when abundance in complex mixture is low. Derivatization strategies have been employed to overcome these challenges; namely the use of acid mediated cleavage ${ }^{9,10}$ or iodoacetalization ${ }^{11,12}$ of the vinyl ether functional groups. The former reaction results in the cleavage of the chain at $s n-1$ reducing the plasmalogen to a lyso species and can be identified by comparison to an untreated sample. The latter strategy uses the innate elevated nucleophilicity of vinyl ethers (relative to non-O-linked alkenes) to facilitate formation of an iodonium species, which is then trapped by $\mathrm{MeOH}$. This chemoselectively labels plasmalogen species and separates them by mass from any isobaric species present.

Previously, we have shown the usage of ${ }^{13} \mathrm{C}$-diazomethane $\left({ }^{13} \mathrm{C}\right.$-DZM) to enhance sensitivity of GPLs in a procedure we described as trimethylation enhancement using diazomethane (TrEnDi). ${ }^{13}$ This approach converts all PE and PC lipids to species bearing a fixed, permanent net-positive charge by methylation of phosphate and amine functionalities of phosphatidylethanolamine moieties. Sensitivity of modified lipids were shown to increase substantially compared to unmethylated lipids, where on average PC species showed 2.4-fold increase and PE species showed 10.7-fold increase when analyzed by LC-MS ${ }^{2} .{ }^{13} \mathrm{C}$-labeled DZM is necessary for the differentiation of PC and PE lipid species that would result in isobaric products if unlabeled DZM was used. Modification of GPLs using diazomethane is very rapid and high yielding (greater than 99\%) when an 
appropriate amount of $\mathrm{HBF}_{4} \cdot \mathrm{Et}_{2} \mathrm{O}$, a strong, non-nucleophilic acid is added. However, during the reactions of lipid extract we noted the appearance of lower mass (400-600 Da) products forming and hypothesized that due to the low $\mathrm{pH}$, many of the plasmalogens in the sample were degrading to their lyso counterparts. ${ }^{13}$ Here we report an optimized TrEnDi derivatization strategy that encapsulates the analysis of plasmalogen lipids by LCMS and show that it is compatible with previously reported plasmalogen identification protocols.

\subsection{Experimental}

Chemicals and Materials. Ammonium acetate, $\mathrm{HBF}_{4} \cdot \mathrm{Et}_{2} \mathrm{O}$ was purchased from SigmaAldrich (St. Louis, MO, USA); LCMS grade isopropanol, methanol, formic acid, and water were purchased from Fisher scientific (Hampton, NH, USA); PE(16:0/18:1), PE(18p/18:1), PC(18:1/14:0), PC(18p/22:6), PC(11:0/11:0) and bovine liver extract were obtained from Avanti Polar Lipids Inc. (Alabaster, Alabama, USA); $\mathrm{KOH}$ and $\mathrm{Et}_{2} \mathrm{O}$ were purchased from Caledon Laboratories Ltd. (Georgetown, ON, Canada).

${ }^{13}$ C-Diazomethane Production. CAUTION: Diazomethane is highly toxic and shock explosive. All reactions involving the preparation and use of diazomethane should be performed in a chemical fumehood with a polycarbonate blast shield placed between the experiment appartus and the operator. Diazomethane solutions should be stored at $-20^{\circ} \mathrm{C}$ in a securely sealed glass container (free of scratches and chips) in an explosion resistant freezer. The production of ${ }^{13} \mathrm{C}$-diazomethane and its precursor $N-\left({ }^{13} \mathrm{C}\right)$ methyl- $N$-nitroso- $p$ toluenesulfonamide have been previously described. ${ }^{13-15}$ 
In solution Chemical Derivatization. To $40 \mu \mathrm{L}$ of $0.5 \mathrm{mg} / \mathrm{ml}$ solution of BLE was added $10 \mu \mathrm{L}$ of a freshly prepared solution of tetrafluoroboric acid diethyl etherate $(50 \mathrm{mM}$, $\mathrm{MeOH})$. Following acidification, $\sim 0.5-0.75 \mathrm{~mL}$ of ethereal ${ }^{13} \mathrm{C}$-diazomethane was immediately added to acidified lipid solution. Any residual diazomethane was removed by a gentle stream of $\mathrm{N}_{2}$ gas such that no yellow color remained and then $200 \mu \mathrm{L}$ of a $10 \mathrm{mM}$ solution of ammonium formate in methanol:isopropanol (1:3, v/v) was added. Finally, after removal of volatiles via a stream of $\mathrm{N}_{2}$ gas the modified lipid extract was resuspended in $10 \mathrm{mM}$ ammonium formate in methanol:isopropanol $(1: 3, \mathrm{v} / \mathrm{v})$ containing $0.5 \mu \mathrm{M}$ PC 11:0/11:0 as an external standard.

Cleavage of vinyl ether linked GPLs with formic acid. To a dry lipid sample was added $50 \mu \mathrm{L}$ of a freshly prepared solution of formic acid and methanol $(4: 1 \mathrm{v} / \mathrm{v})$. The solution was then agitated with a vortex mixer for $1 \mathrm{~min}$ before the addition of $200 \mu \mathrm{L}$ of $10 \mathrm{mM}$ ammonium formate in methanol:isopropanol $(1: 3, \mathrm{v} / \mathrm{v})$ and evaporation of volatiles via a stream of $\mathrm{N}_{2}$ gas.

Iodoacetaliztion. To $2 \mathrm{~mL}$ of an ice-cold solution of $\mathrm{NH}_{4} \mathrm{CO}_{3} \mathrm{H}$ in $\mathrm{MeOH}(8.6 \mathrm{mM})$ was added $1 \mathrm{~mL}$ of an $\mathrm{I}_{2}$ solution in $\mathrm{CHCl}_{3}(2.3 \mathrm{mM})$. Then, $50 \mu \mathrm{L}$ of the $\mathrm{NH}_{4} \mathrm{CO}_{3} \mathrm{H} / \mathrm{I}_{2}$ solution was added to a dry lipid sample and the resulting mixture was allowed to react on ice for 5 min before evaporation of volatiles via a stream of $\mathrm{N}_{2}$ gas. In general methylation with ${ }^{13} \mathrm{C}$ DZM was preformed prior to iodoacetalization.

LCMS Analysis. A $2 \mu \mathrm{L}$ aliquot (10 $\mu \mathrm{L}$ for lipid standards) of $0.5 \mathrm{mg} / \mathrm{ml}$ bovine liver extract was injected using auto sampler of an Agilent 1260 Infinity II separated with an Agilent Poroshell 120 column (EC-C18 $2.7 \mu \mathrm{m}, 2.1 \times 100 \mathrm{~mm}$ ). The column was maintained at $45{ }^{\circ} \mathrm{C}$ and a constant flow rate of $0.4 \mathrm{~mL} / \mathrm{min}$. The mobile phases consisted 
of (A) 50:50 (v/v) methanol:water with ammonium formate $(10 \mathrm{mM})$ and (B) 75:50 (v/v) methanol:isopropanol with ammonium formate $(10 \mathrm{mM})$. The separation gradient was 0 $\min 0 \%(\mathrm{~B}) ; 1.0 \min 30 \%(\mathrm{~B}) ; 3.5-10.0 \min 86 \%(\mathrm{~B}) ; 11-25 \min 100 \%$ (B). The eluent was introduced into an Agilent 6546 Q-TOF using the parameters: MS-ESI polarity positive (m/z 100 - 1700); gas temp, $200{ }^{\circ} \mathrm{C}$; drying gas, $10 \mathrm{~L} / \mathrm{min}$; nebulizer, $50 \mathrm{psi}$; sheath gas temp, $300{ }^{\circ} \mathrm{C}$; sheath gas flow, $12 \mathrm{~L} / \mathrm{min}$; VCap, $3500 \mathrm{~V}$; nozzle voltage, $0 \mathrm{~V}$; fragmentor, $150 \mathrm{~V}$; skimmer, $65 \mathrm{~V}$; oct $1 \mathrm{Vpp}, 750 \mathrm{~V}$. MS 2 -ESI polarity positive, Auto (m/z 40 - 1700); Isolation width, narrow; static exclusion range, m/z 40 - 300; iterative, $\pm 20 \mathrm{ppm}, \pm 0.2 \mathrm{~min}$ retention time $\left(\mathrm{T}_{\mathrm{R}}\right)$ exclusion; collision energy, $28 \mathrm{eV} . \mathrm{MS}^{2}$-ESI polarity negative (m/z 40 - 1700); VCap, -3000; all other source parameters are the same as ESI positive; collision energy, $25 \mathrm{eV}$; all other $\mathrm{MS}^{2}$ parameters are the same as ESI positive.

Data-Preprocessing with MZmine 2. Agilent data files were converted to .mzdata format using MassHunter Workstation Qualitative analysis before processing with MZmine 2.51 software. ${ }^{16,17}$ All data files were filtered to include m/z $300-1200$ and $4.0-18 \mathrm{~min}$, before mass detection with a noise limit of 1000 . Features were detected with a minimum time span of $0.1 \mathrm{~min}$, minimum height of 1000 and $\mathrm{m} / \mathrm{z}$ tolerance of 0.01 and $0.0 \mathrm{ppm}$. Chromatogram deconvolution was achieved with a chromatographic threshold of $90 \%$, minimum RT range of 0.1 min, minimum relative height of $5 \%$, minimum absolute height of $1.0 \times 10^{4}$, ratio of peak top/edge of 1.7 and a peak duration of $0.0-10.0 \mathrm{~min}$. Deconvoluted peak list was deisotoped using a m/z $0.01(0.0 \mathrm{ppm})$ tolerance and a RT tolerance of 0.1 $\mathrm{min}$. Each triplicate was then aligned with $\mathrm{m} / \mathrm{z}$ tolerance of $0.01(0.0 \mathrm{ppm})$, weight for $\mathrm{m} / \mathrm{z}$ 20, RT tolerance $0.2 \mathrm{~min}$, and weight for RT 10 . 


\subsection{Results and Discussion}

Acid-mediated cleavage of plasmenyl ether-linked glycerophospholipids can be advantageous since their vinyl ether functionality can be used as a handle for detection. A current strategy for detecting plasmenyl lipids is to treat a portion of a sample with acid and compare MS results of treated and non -treated samples. Through the absence of a lipid species in an extract treated with acid, compared to that of an untreated sample indicates a plasmenyl linked lipid species. However, the acid labile linkage can undergo cleavage prior to MS analysis. Such as, in acidic cell growth media, acidic extraction protocols or during chromatography using overly acidic eluents.

In Figure 3.4.1b, the chromatograms show the complete cleavage of two plasmenyl ether lipids, $\mathrm{PE}(\mathrm{P}-18: 0 / 18: 1)$ and $\mathrm{PC}(\mathrm{P}-18: 0 / 22: 6)$, in a sample treated with $80 \%$ formic acid in methanol for 1 min. The signal of other diacyl GPL standards in the mixture, $\mathrm{PE}(16: 0 / 18: 1)$ and $\mathrm{PC}(18: 1 / 14: 0)$, remain intact by the acidic conditions. Prior to this report, TrEnDi method used the addition of a small amount of diethyl ether with saturated $\mathrm{HBF}_{4} \bullet \mathrm{Et}_{2} \mathrm{O}$. We hypothesized that due to the large molar excess of acid that was used the plasmenyl ether-linked lipids were being cleaved to produce lyso species (Scheme 3.4.1). Figure 3.4.1c depicts the reaction of $\mathrm{DZM}$ and $\mathrm{HBF}_{4}$ with $\mathrm{PC}$ and $\mathrm{PE}$ standards and demonstrates the absence of the expected methylated plasmenyl standards. No traces of unmethylated standards could be observed during the analysis (Figure S1), indicating that diacyl PC and PE were completely converted to methylated products, and PC and PE plasmenyl ethers were cleaved during the reaction. 
a)

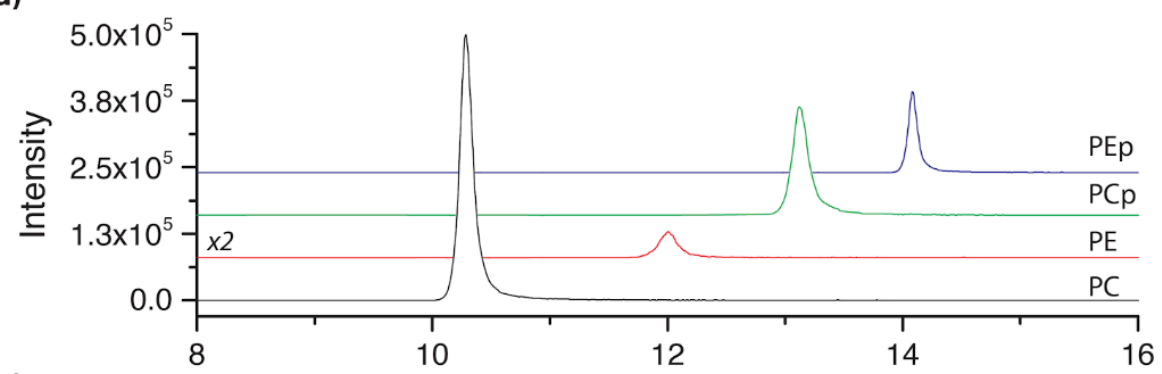

b)
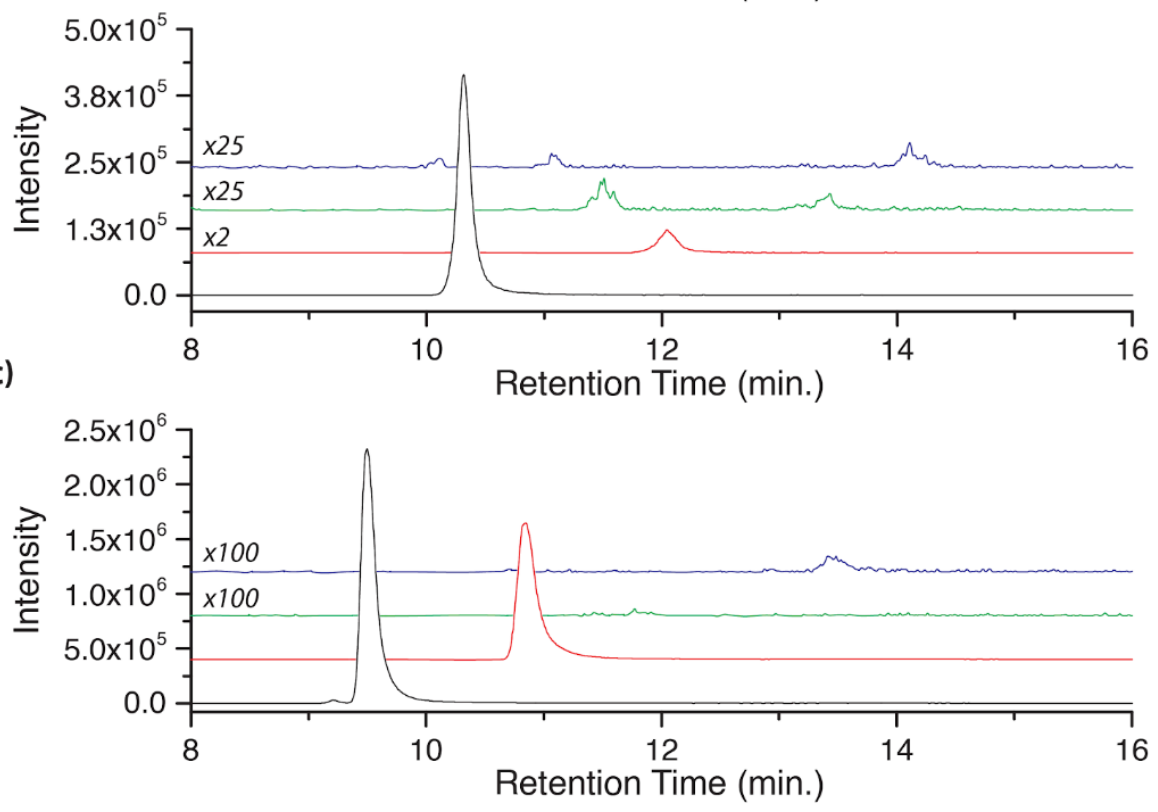

Figure 3.4.1 LCMS analysis showing XIC traces of (a) un-treated lipid standards PE(16:0/18:1), PE(P-18:0/18:1), PC(18:1/14:0), PC(P-18:1/14:0), (b) treated with $80 \%$ formic acid in methanol, (c) treated with unoptimized TrEnDi conditions. Chromatograms are stacked with a Y-offset to clearly show each peak 


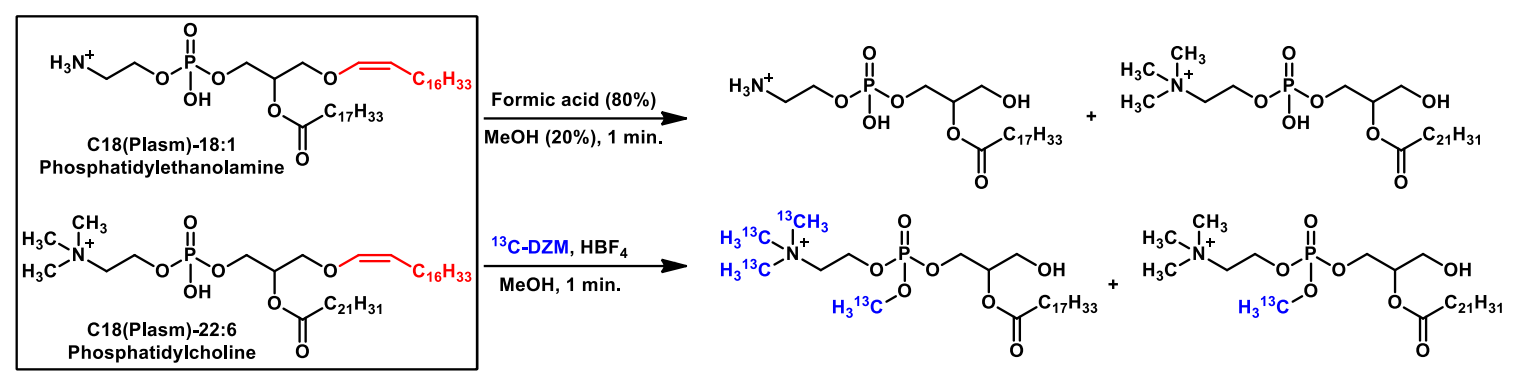

\section{Scheme 3.4.1 Cleavage of plasmenyl ether lipids via formic acid and unoptimized TrEnDi reaction conditions}

By using a known concentration of $\mathrm{HBF}_{4}$ in methanol, rather than a saturated amount from a partially miscible mixture of $\mathrm{HBF}_{4}$ in $\mathrm{Et}_{2} \mathrm{O}$, we could begin to optimize reaction conditions of TrEnDi to preserve the plasmalogens. Table 3.4.1 shows the effect of $\left[\mathrm{HBF}_{4}\right]$ on the methylation of plasmenyl ether-linked PC and PE standards. At lower $\left[\mathrm{HBF}_{4}\right]$ we observe a low conversion to fully methylated products. In general, $\mathrm{PE}(\mathrm{P}-$ 18:0/18:1) showed much lower conversion percentages than $\mathrm{PC}(\mathrm{P}-18: 0 / 22: 6)$ due to $\mathrm{PE}$ having four sites of methylation thus requiring four times more acid than PC. The two plasmenyl standards were found to be completely methylated at both $7.1 \mathrm{mM}$ and $10 \mathrm{mM}$ $\mathrm{HBF}_{4}$, with little to no loss due to cleavage of the vinyl ether groups. Going forward, we decided that $10 \mathrm{mM} \mathrm{HBF}_{4}$ would be more amenable to a complex lipid extract due to the higher concentrations of phospholipids that would need to be protonated. Similar concentrations of $\mathrm{HBF}_{4}$ etherate were used to derivatize HeLa cell extract with DZM to determine if the optimized conditions would scale to a complex mixture (Figure S2). The concentration of unmodified HeLa extract where the top five PC peaks saturated the detector was determined. This concentration was further diluted $3 \mathrm{x}$ to account for the signal enhancement of PCs provided via TrEnDi. All optimization experiments were performed 
at this concentration. Even though different samples may differ in the total lipid content; the derivatization conditions were effective in fully derivatizing different lipid extracts when the optimal injection concentration of samples was found (the concentration that maximized signal without saturating the detector). The successful derivatization of different lipid extracts such as BLE is discussed in following sections. From the HeLa cell derivatization trials, $10 \mathrm{mM} \mathrm{HBF}_{4}$ could be used to methylate complex lipid samples, but an $\mathrm{HBF}_{4}$ concentration of $960 \mathrm{mM}$ was found to decrease signal intensities drastically.

Table 3.4.1 Optimization of $\mathrm{HBF}_{4}$ etherate concentration for the complete methylation of lipid standards

\begin{tabular}{ccc}
\hline & \multicolumn{2}{c}{$\begin{array}{c}\text { Conversion to fully } \\
\text { methylated product (\%) }\end{array}$} \\
\hline $\begin{array}{c}{\left[\mathrm{HBF}_{4}\right]} \\
(\mathrm{mM})\end{array}$ & $\mathrm{PE}(\mathrm{P}-36: 1)$ & $\mathrm{PC}(\mathrm{P}-40: 6)$ \\
\hline 0.5 & 5.1 & 18.8 \\
1.4 & 19.3 & 51.4 \\
2.9 & 93.4 & 99.6 \\
4.8 & 96.5 & 100 \\
7.1 & 100 & 100 \\
10.5 & 100 & 100
\end{tabular}

One other modification to the TrEnDi protocol, made to ensure the survival of plasmenyl ether lipids, was the addition of an aliquot of $10 \mathrm{mM}$ ammonium formate to the lipid solution one minute after the DZM was added (Scheme 3.4.2). Thus, any excess acid would be minimized during the sample preparation stage before LCMS analysis and would limit the time for degradation. Figure 3.4.2a shows the complete methylation of both diacyl and plasmenyl ether lipids. Based on peak area normalized to the external standard 
PC(11:0/11:0), fold increase of the TrEnDi derivatized standards compared to their unmethylated counterparts were observed to be $\mathrm{PC}(18: 1 / 14 ; 0)$ : 5.3 fold, $\mathrm{PE}(16: 0 / 18: 1)$ : 36.8 fold, $\mathrm{PC}(\mathrm{P}-18: 0 / 22: 6): 4.5$ fold, $\mathrm{PE}(\mathrm{P}-18: 0 / 18: 1): 27.8$ fold. The increase in signal of both PE and PC classes are consistent with previous findings and the new conditions do not affect the reaction with diacyl GPL standards. Figure 3.4.2b directly compares the TIC of a $2 \mu \mathrm{L}$ injection of a $0.5 \mathrm{mg} / \mathrm{ml}$ solution of bovine liver extract (BLE) with an equivalent aliquot that has been methylated with DZM. The overall signal from the BLE treated with DZM and $10 \mathrm{mM} \mathrm{HBF}_{4}$ is shown to be much greater than that of the unmethylated sample and directly demonstrates how TrEnDi modification of complex mixture of lipids increases the signal in MS globally.

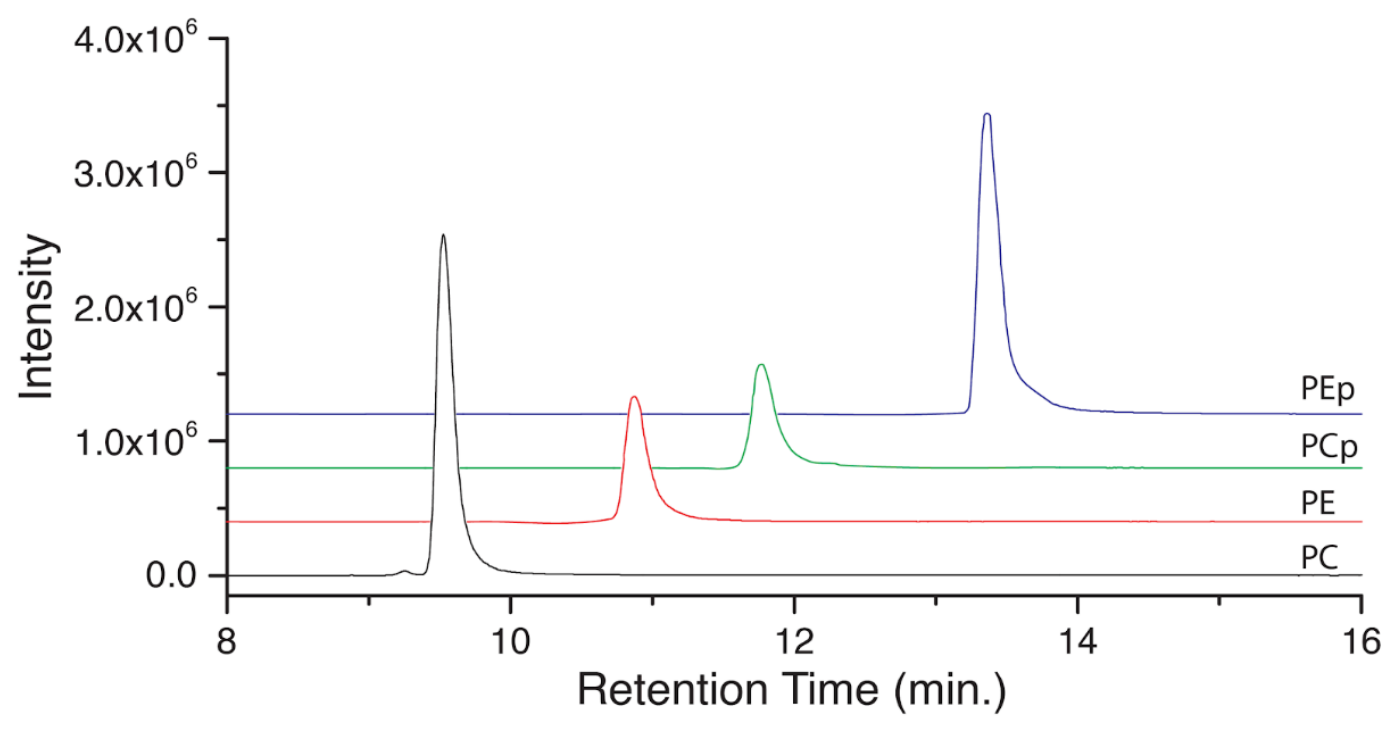

Figure 3.4.2 Lipid standards treated with ${ }^{13} \mathrm{C}-\mathrm{DZM}$ and $10 \mathrm{mM} \mathrm{HBF}_{4}$ etherate 


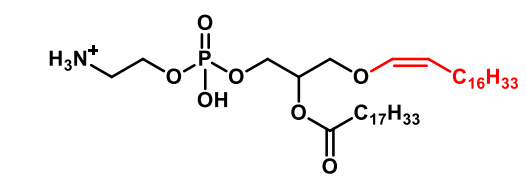

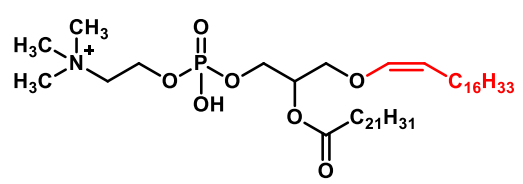

$\underset{\text { 2. } 10 \mathrm{mM} \mathrm{NH}_{4} \mathrm{CO}_{2} \mathrm{H}, \mathrm{MeOH}, \mathrm{IPA}}{\stackrel{13}{\mathrm{C}-\mathrm{DZM}, 10 \mathrm{mM} \mathrm{HBF}} \text {, MeOH, } 1 \mathrm{~min} .}$
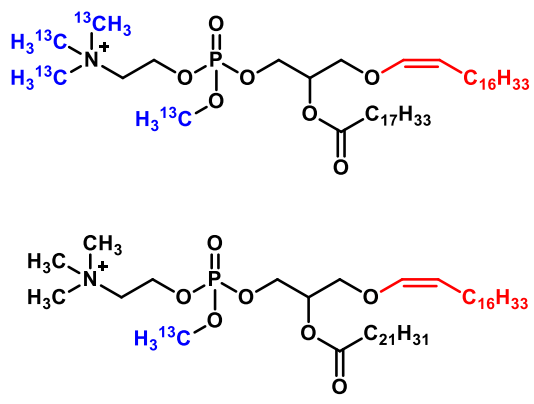

Scheme 3.4.2 Methylation of PC and PE plasmalogen standards with ${ }^{13} \mathrm{C}-\mathrm{DZM}$ and $10 \mathrm{mM} \mathrm{HBF}_{4}$ produces methylated species with no cleavage of vinyl ether

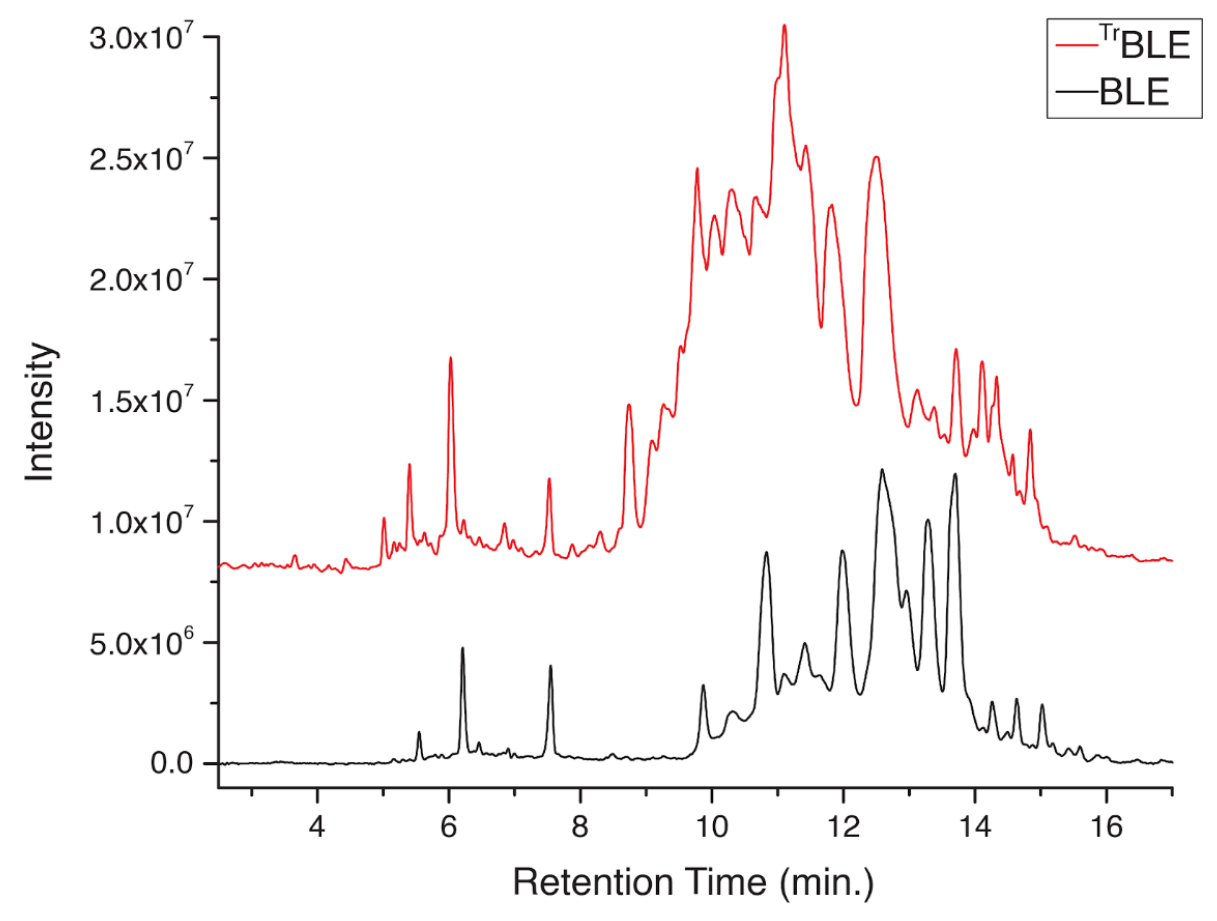

Figure 3.4.3 Overall signal increase in the TIC of TrEnDi methylated BLE ( $\left.{ }^{\operatorname{Tr} B L E}\right)$ over native lipids in the LCMS analysis of bovine liver extract. ${ }^{\text {TrBLE }}$ is stacked by Y offset of $6.0 \times 10^{6} \mathrm{cps}$ 
An analysis of plasmenyl ether lipids in BLE was preformed through the comparison of a sample treated with $80 \%$ formic acid against an untreated sample. Features were detected and deconvoluted with MZmine 2 with conditions found to work well for lipodomic dataset analysis. ${ }^{17}$ Any features that were observed to decrease in normalized peak area by $50 \%$ or greater were identified as vinyl ether-containing lipids. The threshold of $50 \%$ was chosen to have higher confidence in the plasmalogens identity. A total of twenty-nine plasmalogen lipids present in BLE were identified using these parameters. These plasmenyl ether linked lipids were used to monitor the effectiveness of our new optimized TrEnDi reaction methodology.

Figure 3.4.3a shows the extracted ion chromatogram (EIC) of $\mathrm{m} / \mathrm{z} 752.6$ from untreated BLE, which could be identified by exact mass as any of the following isomers: PE(P-38:4), $\mathrm{PE}(\mathrm{O}-38: 5)$, $\mathrm{PC}(\mathrm{P}-35: 4)$ or $\mathrm{PC}(\mathrm{O}-35: 5)$. Four chromatographically distinct signals were observed for $\mathrm{m} / \mathrm{z} 752.6$ at $10.4,10.7,13.1$ and $13.4 \mathrm{~min}$. Through the treatment with formic acid, a large decrease in peak area could be observed for each signal, indicating that these peaks are predominantly plasmalogen lipids. The remaining areas do indicate there are co-eluting and isomeric plasmanyl counterparts to the identified plasmalogens. Following TrEnDi modification of BLE with optimized conditions, the two co-eluting isomers with $\mathrm{m} / \mathrm{z} 767.6$ (Figure 3.4.4b) were observed to have similar peak shapes to the first pair of signals in Figure 3.4.4a. and corresponds to a mass shift of $15.0 \mathrm{Da}$ (corresponding to addition of on ${ }^{13} \mathrm{CH}_{2}$ ). Furthermore, $\mathrm{m} / \mathrm{z} 812.6$ was observed in TrEnDi modified BLE, as a mass shift of $60.1 \mathrm{Da}$ (corresponding to the addition of four ${ }^{13} \mathrm{CH}_{2}$ ) and had similar peak shape to the pair of underivatized signals at 13.1 and 13.4 min in Figure 3.4.4a. From the respective mass shifts of 15.0 and $60.1 \mathrm{Da}$, matching of the exact mass to 
lipid map structure database, ${ }^{18}$ and the absence of peaks after exposure to $80 \%$ formic acid we concluded that the first pair of signals in Figure 3.4.4a arise from $\mathrm{PC}(\mathrm{P}-35: 5)$ and the second pair is $\mathrm{PE}(\mathrm{P}-38: 4)$. The presence of methylated $\mathrm{PE}(\mathrm{O}-38: 5)$ was clearly observed with a retention time $\left(T_{R}\right)$ of 11.1 min (Figure 3.4.4c) and was unaffected by the acid treatment. The plasmanyl ether PE was present in the unmethylated sample but was overlapped to a larger degree by other species with $\mathrm{m} / \mathrm{z} 752.6$, making it more challenging to identify. In this case, methylated $\mathrm{PC}(\mathrm{P}-35: 5)$ and $\mathrm{PE}(\mathrm{P}-38: 4)$, when normalized to an external standard showed the increase in signal of 3.8 and 24.8 -fold respectively.
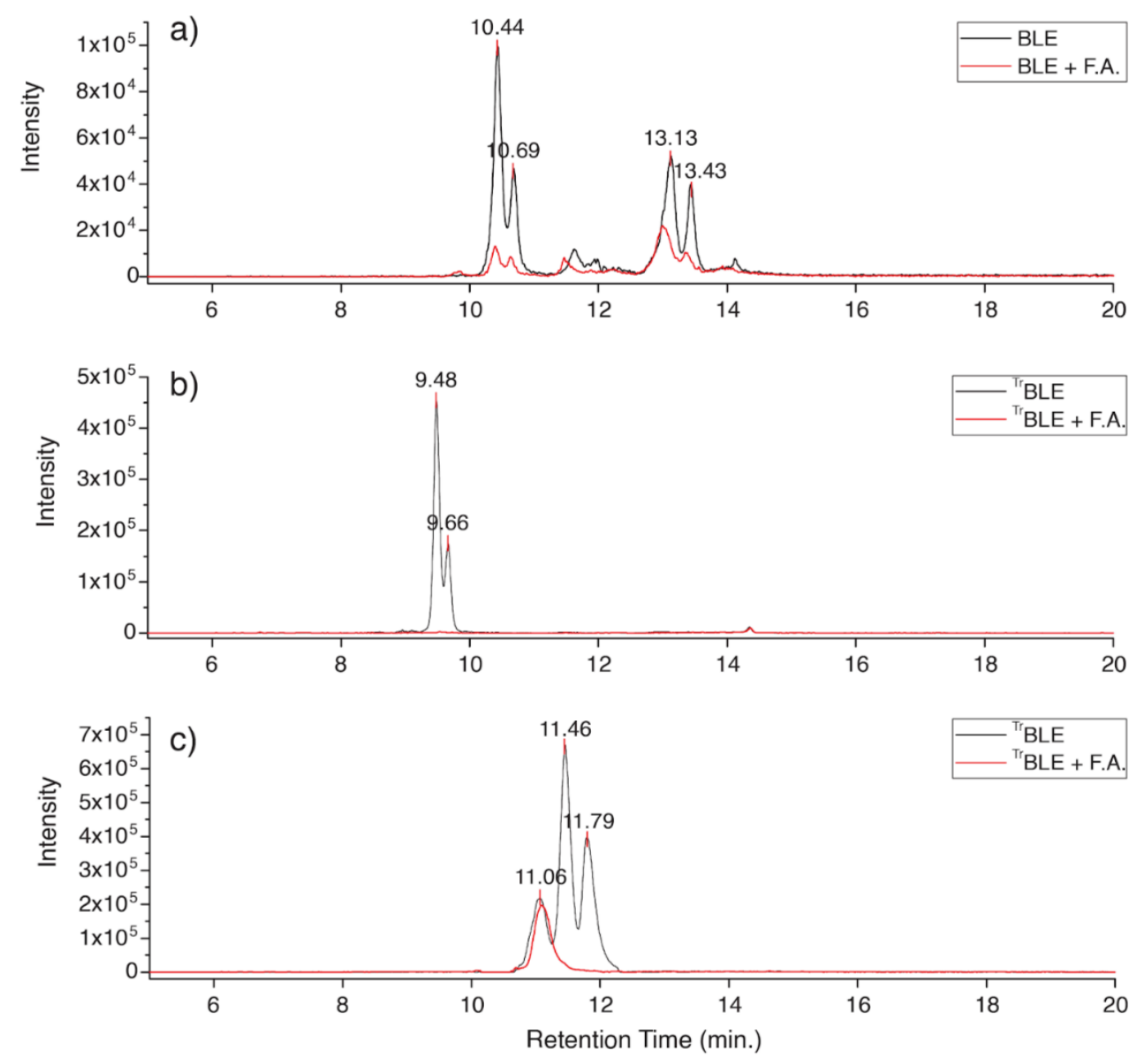

Figure 3.4.4 LCMS analysis showing extracted ion chromatograms for (a) an unmodified lipid species in untreated BLE with $\mathbf{m} / \mathbf{z} 752$, (b) lipid species from the 


\section{TrEnDi modified BLE with $\mathrm{m} / \mathrm{z} 767$, and (c) lipid species from the TrEnDi modified BLE with $\mathbf{m} / \mathbf{z} 812$}

The 29 plasmalogens found in the underivatized BLE could also be observed in the TrEnDi-modified material as either $[\mathrm{M}+15 \mathrm{Da}]^{+}$or $[\mathrm{M}+60 \mathrm{Da}]^{+}$identifying each as belonging to PC or PE class, respectively. Figure 3.4.5 is the comparison of normalized peak intensities of underivatized versus methylated plasmalogens found in the BLE. The peak areas were normalized to external standard $\mathrm{PC}(22: 0)$ and each derivatization reaction was performed in chemical triplicates. In general, the peak intensities of TrEnDi-modified lipids were significantly greater than the corresponding underivatized lipids: 22 plasmenyl lipids were identified as PCs and afforded an average of 3.0-fold increase and 7 plasmenyl lipids were identified as PEs and yielded an average of 29.3-fold increase. The optimized TrEnDi conditions when applied to BLE successfully allowed for the analysis and identification by mild acid methanolysis of plasmalogens.

Kendrick mass plots are a useful way to showcase relationships within a grouping of lipid masses belonging to a GPL class by plotting the Kendrick nominal mass (KNM) against the Kendrick mass defect (KMD) of a methylene unit $\left(\mathrm{CH}_{2}\right)$. The main purpose of plotting lipid data this way is to represent the mass of methylene units in the KNM while the contribution of any heteroatoms and degrees of unsaturation can be depicted by the KMD value. ${ }^{19}$ In Figure 3.4.6, each unmodified lipid mass is converted to its Kendrick nominal mass and plotted against its Kendrick mass defect. Once arranged in this way groupings of lipids with the same bulk number of methylene units can be observed. For example $\mathrm{m} / \mathrm{z} 686.5(\mathrm{KNM}=685)$, identified by exact mass using the LIPID MAPS 
Structure Database (LMSD) ${ }^{18}$ as $\mathrm{PE}(\mathrm{P}-33: 2)$ or $\mathrm{PC}(\mathrm{P}-30: 2)$, is related by KMD to $\mathrm{m} / \mathrm{z}$ $700.5(\mathrm{KNM}=699)$ and could be identified as PE(P-34:2) or PC(P-31:2). Furthermore, a species with $\mathrm{m} / \mathrm{z} 704.6(\mathrm{KNM}=703)$ can be identified using LMDS as PE(P-34:0) or $\mathrm{PC}(\mathrm{P}-31: 0)$, is related to $\mathrm{m} / \mathrm{z} 702.5$ since $\Delta \mathrm{KMD}=0.013(\mathrm{KMD}$ of $2 \mathrm{H})$ and can be identified as $\mathrm{PE}(\mathrm{P}-34: 1)$ or $\mathrm{PC}(\mathrm{P}-31: \mathbf{1})$. When methylated lipid species were arranged in a Kendrick mass plot the same groupings arose. In Figure 3.4.7 PlsEtn and PlsCho could be differentiated by their mass shift of 60 and $15 \mathrm{Da}$ when compared to unmodified species and were plotted accordingly.

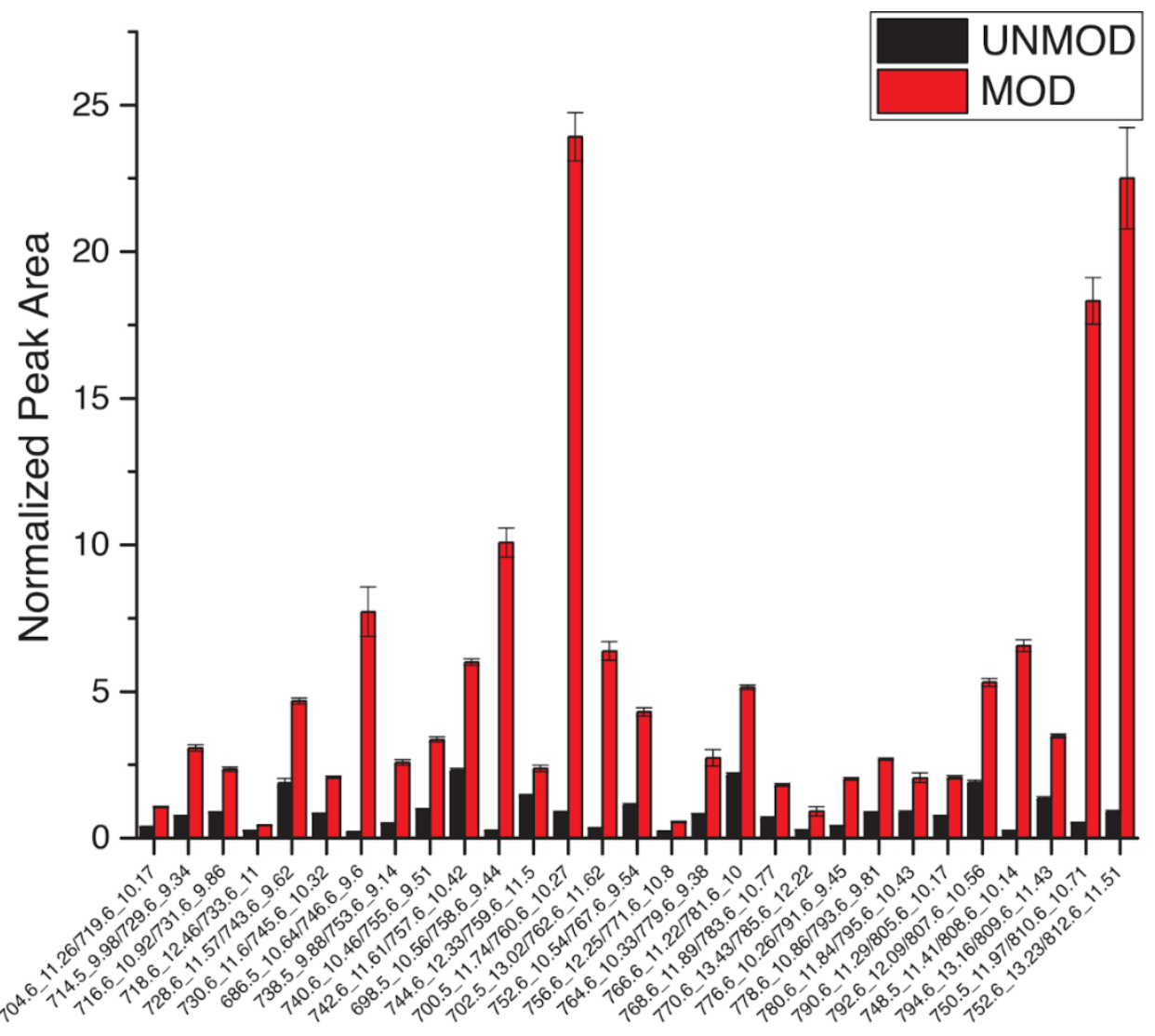

Figure 3.4.5 Plasmanyl ether lipids detected in unmodified BLE (black) and methylated lipids from the TrEnDi derivatized BLE (red), where error bars showing relative standard error of the average of three sequential injections (BLE) 
of chemical triplicates ( $\left.{ }^{T r} \mathrm{BLE}\right)$. The $\mathrm{X}$ axis titles are formatted as $\mathbf{m} / \mathbf{z}_{\mathrm{BLE}} \mathbf{T}_{\mathrm{R} \text { BLE }} /$ $\mathbf{m} / \mathbf{z}^{\mathrm{Tr}}{ }_{\text {BLE__ }} \mathbf{T}_{\mathbf{R}}{ }^{\mathrm{Tr}}{ }_{\text {BLE. }}$

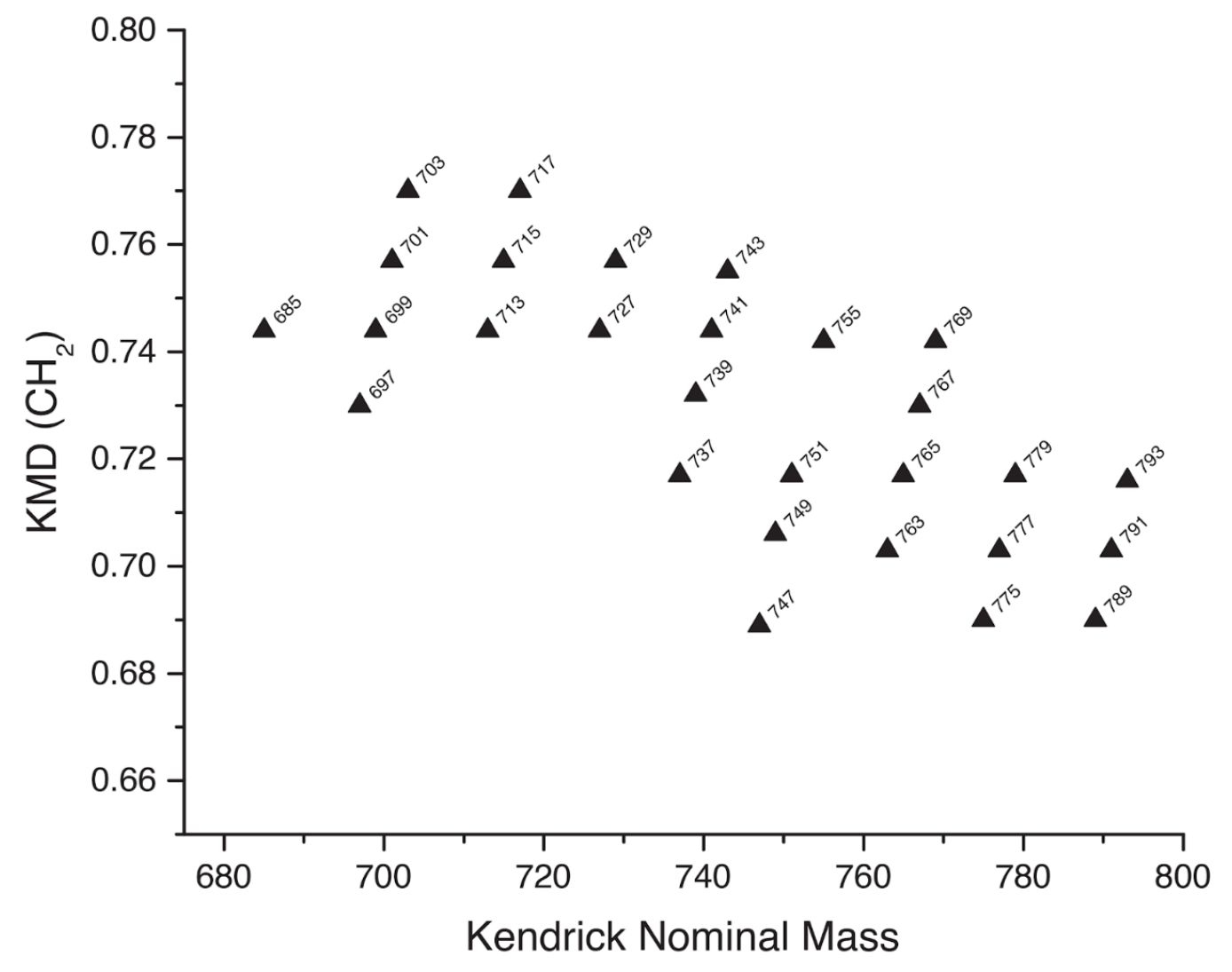

Figure 3.4.6 Kendrick Mass plot with $\mathrm{CH}_{2}$ Base of Unmodified Plasmenyl Etherlinked GPLs Identified by the Mild Acid Cleavage of BLE 


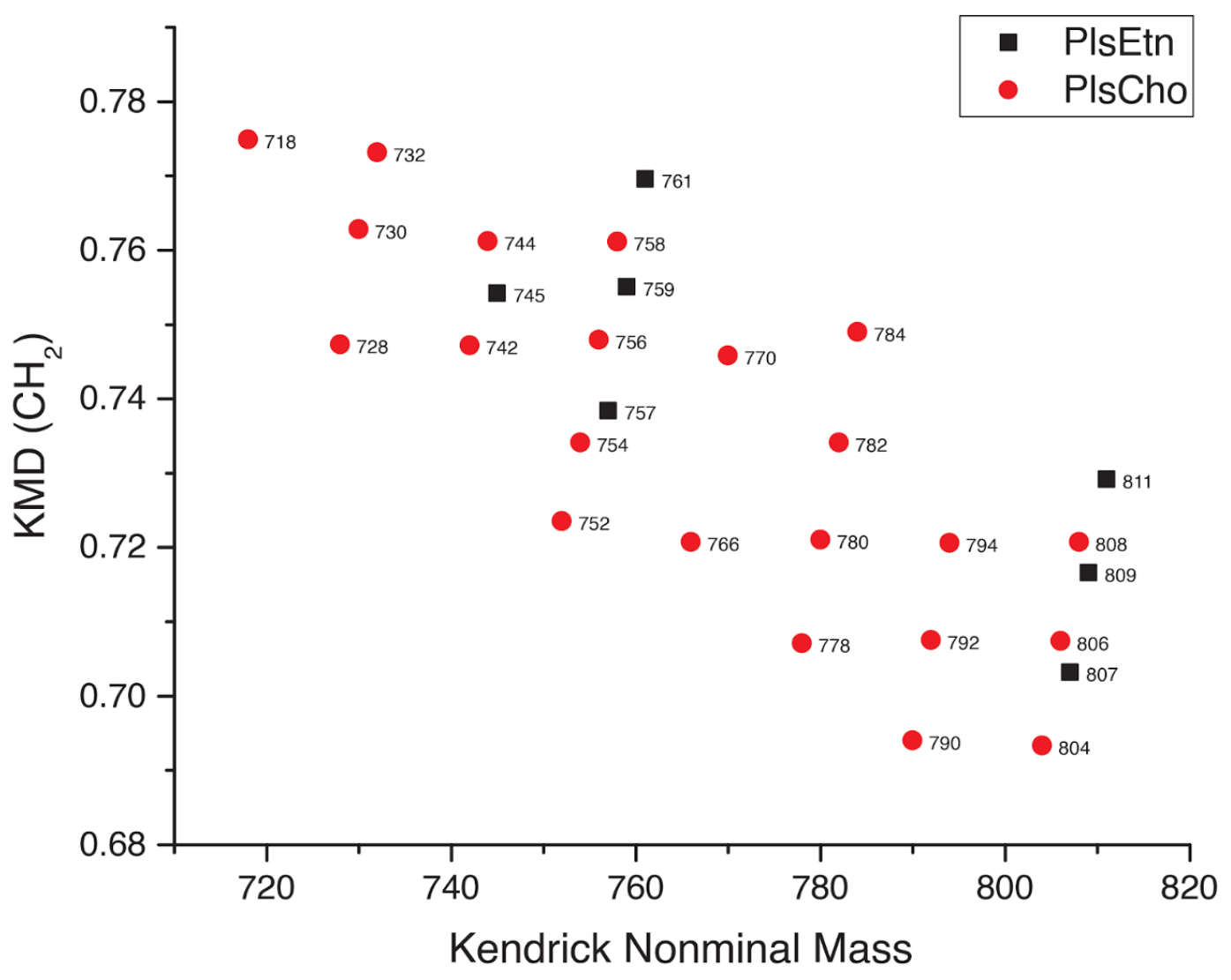

Figure 3.4.7 Kendrick Mass Plot with $\mathrm{CH}_{2}$ Base of Methylated Plasmenyl Etherlinked GPLs Identified by the Mild Acid Cleavage of ${ }^{13} \mathrm{C}-T r E n D i-B L E$

Table 3.4.2 is a comparison of data from the TrEnDi modification of BLE standards for the identification and relative quantitation of plasmalogen lipids. The average relative standard deviation modified lipid peak area was found to be 3.81\% (PlsCho) and 5.63\% (PlsEtn) over three chemical reactions. The unmodified errors associated with PE and PC plasmalogens were obtained by triplicate injections of the same solution and represent instrument error. The reproducibility of optimized methylation conditions is comparable to our previous studies on HeLa cell extract. The average decrease in peak area of plasmalogens compared to their formic acid treated counterparts was found to be $15 \%$ 
greater in the TrEnDi modified samples. The larger differences in peak area after acid treatment can be attributed to the chromatographic separation of modified plasmanyl and plasmanyl ethers (Figure 3.4.4) and will ultimately aid in the accurate identification low abundance of plasmalogens using our methodology. The fold increase of methylated lipids, especially PE plasmalogens, will also aid in the detection, identification and quantification of low-abundance ether lipids, which could go unidentified in complex biological samples.

Table 3.4.2 Summary of the Comparison of TrEnDi Modified Plasmalogens to Underivatized Identified in BLE

\begin{tabular}{ccccc}
\hline & \multicolumn{2}{c}{${ }^{\text {TrBLE }}$} & \multicolumn{2}{c}{ BLE } \\
\hline & PlsCho & PlsEtn & PlsCho & PlsEtn \\
\hline $\begin{array}{c}\text { Average Normalized } \\
\text { Peak Area }\end{array}$ & 2.78 & 13.64 & 0.99 & 0.48 \\
$\begin{array}{c}\text { Relative Standard } \\
\text { Deviation (\%) }\end{array}$ & 3.81 & 5.63 & 4.42 & 2.91 \\
$\begin{array}{c}\text { Average Difference in } \\
\text { Peak Area to Acid } \\
\text { Treated Sample (\%) }\end{array}$ & 98.86 & 95.83 & 84.08 & 80.76 \\
& & & & \\
Average Fold Increase & 2.81 & 28.1 & - & -
\end{tabular}

Another derivatization technique to identify plasmalogens is the conversion of the vinylic ether to the corresponding $\alpha$-iodo methyl acetal via reaction with methanolic $\mathrm{I}_{2}$ and $\mathrm{NH}_{4} \mathrm{CO}_{3} \mathrm{H}$ (Scheme 3.4.3). Recently, this technique has been performed by Reid and coworkers in conjunction with another derivatization strategy to prepare $d_{6}-S, S^{\prime}$ dimethylthiobutanoylhydroxysuccinimide esters to analyze plasmalogen lipids via a direct injection MS strategy. ${ }^{12}$ To show that ${ }^{13} \mathrm{C}-\mathrm{TrEnDi}$ is compatible with other plasmalogen 
derivatization and identification methodology, we prepared a sample of BLE first by applying the aforementioned optimized methylation procedure immediately followed by $\mathrm{I}_{2} / \mathrm{NH}_{4} \mathrm{CO}_{3} \mathrm{H}$. Derivatization of $\mathrm{BLE}$ with ${ }^{13} \mathrm{C}-\mathrm{DZM}$ was performed prior to iodoacetalization to prevent neutralization of $\mathrm{HBF}_{4}$ from residual $\mathrm{NH}_{4} \mathrm{CO}_{3}$. A few examples of the results of these reactions are found in Figure 3.4.8. We could confirm the iodoacetal product by the shift in mass of $157.9 \mathrm{Da}$ (net addition of $\mathrm{CH}_{3} \mathrm{IO}$ ) when compared to the methylated products.

Figures 3.4.8a-d show EICs of four reaction products of the previously identified plasmalogens from BLE. The overall peak shapes of the extracted chromatograms of derivatized product remained consistent between $\mathrm{DZM}+\mathrm{I}_{2} / \mathrm{MeOH}$ and DZM-only derivatized products. Retention time of the iodoacetalized plasmalogens appeared to increase by approximately $0.5 \mathrm{~min}$, likely due to the addition of the large, non-polar iodine atom overwhelming any slight increase in polarity caused by the methoxy group. Notably, Figures 3.4.8e, f depict two plasmalogens that were not identified in the unmodified BLE. These species were absolutely identified as plasmalogens because of their absence in the samples treated with formic acid and being successfully modified by iodoacetalization. With a closer inspection the six EICs, the signal intensity dropped $20-65 \%$ in normalized peak area after the iodoacetalization was performed. The reason for this is not entirely clear, but it could be attributed to the removal of all plasmanyl ether interference and degradation of the product to its lysed form during chromatography (as acetals are also acid sensitive) thus a shotgun MS analysis would be preferred. Regardless of the decrease in signal, the modification was shown to be compatible with the identification of plasmalogens from a complex mixture using the TrEnDi protocol reported herein. While 
this two-step derivatization strategy may offer an characterization advantage by incorporation of an iodine atom and methyl acetal that eliminates plasmanyl and plasmenyl isobaricity, we submit that the quantitation robustness and simplicity of our singlederivatization strategy outweighs the combination of any such advantages with the ubiquitous but variable decrease in signal intensity.

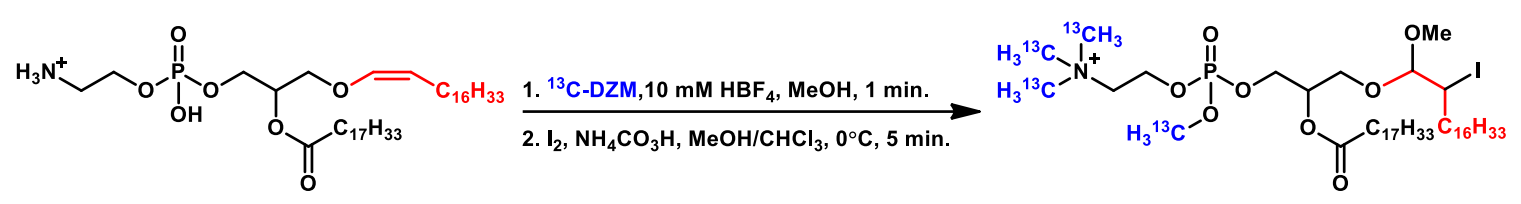

Scheme 3.4.3 Two-step derivatization of plasmalogen lipids using ${ }^{13} \mathrm{C}-\mathrm{DZM}$ and $\mathrm{I}_{2}$ to produce a methylated GPL with fixed positive charge and iodoacetylated vinyl ether 

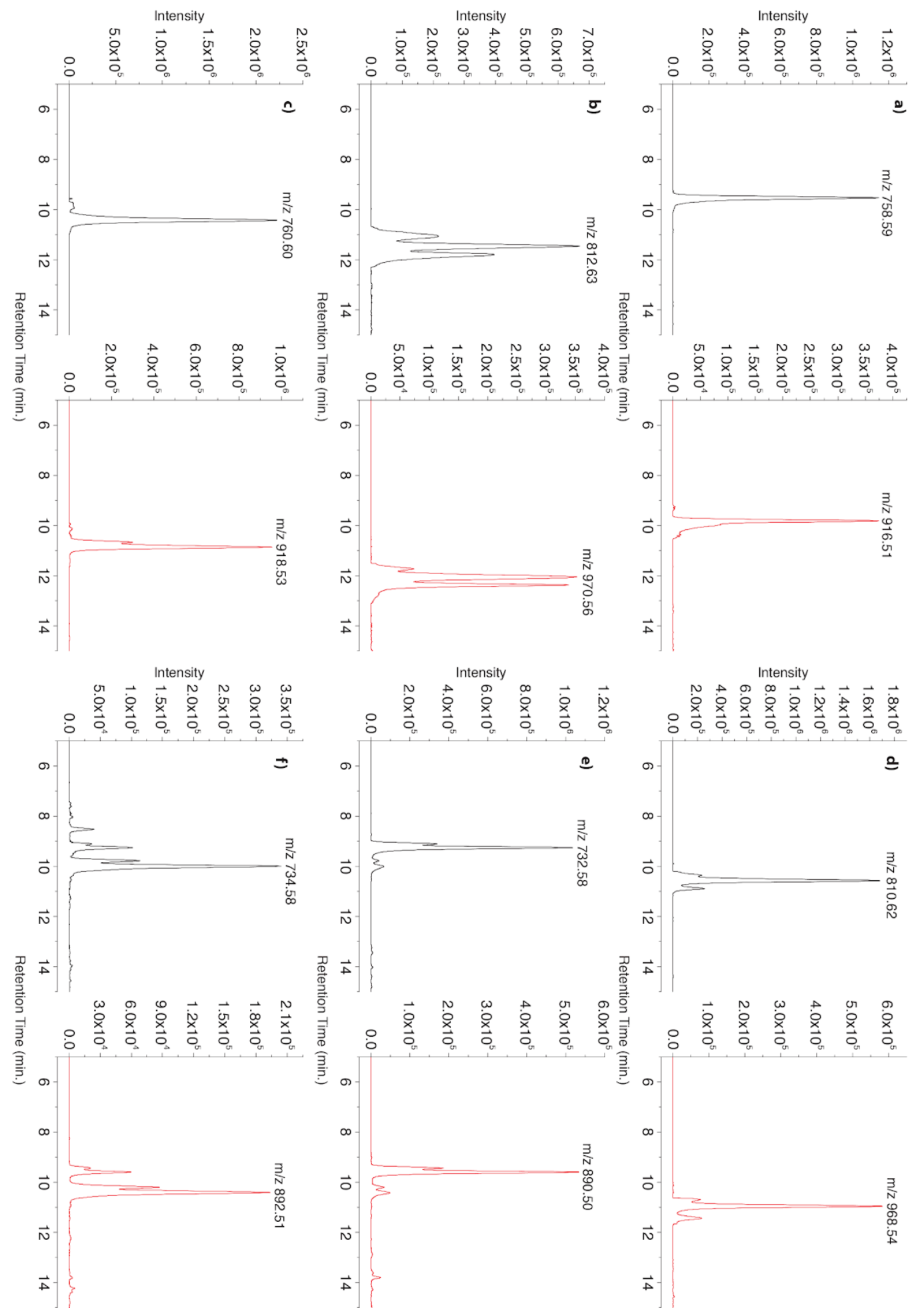

Figure 3.4.8 Plasmalogen Lipids Identified from BLE showing the comparison of the extracted ion chromatograms of TrEnDi derivatized lipids (black) and corresponding $\operatorname{TrEnDi}+\mathbf{I}_{2}$ treated lipids(red) 


\subsection{Conclusion}

The methylation of glycerophospholipids with 13C-DZM offers several promising benefits compared to other derivatization techniques such as, increased signal intensity, no by-products formed, excess reagent is readily removed, and very rapid reaction time. Following our initial investigation on the analysis of GPLs using diazomethane as a derivatizing agent to methylate amine and phosphate functionalities, we have made significant improvements to include the derivatization of plasmalogens. By using a controlled concentration of $\mathrm{HBF}_{4}$ along with two plasmalogen-specific derivatization techniques, we were able to differentiate isobaric plasmanyl and plasmenyl phospholipids and confidently identify twenty-nine plasmalogens in BLE. Compared to non-modified plasmalogens, ${ }^{13} \mathrm{C}$-TrEnDi showed greater difference in peak area when treated with $80 \%$ formic acid in methanol. Furthermore, with modification with TrEnDi has been shown to be a modular way of derivatizing GPLs to boost signal-to-noise ratio of low-abundance lipids species and increase the overall annotation and quantitation of lipids in biologically complex samples.

\subsection{References}

(1) Braverman, N. E.; Moser, A. B. Biochim. Biophys. Acta-Mol. Basis Dis. 2012, $1822(9), 1442-1452$.

(2) Paul, S.; Lancaster, G. I.; Meikle, P. J. Prog. Lipid Res. 2019, 74 (April), 186-195.

(3) Messias, M. C. F.; Mecatti, G. C.; Priolli, D. G.; De Oliveira Carvalho, P. Lipids Health Dis. 2018, 17 (1), 1-12.

(4) Su, X. Q.; Wang, J.; Sinclair, A. J. Lipids Health Dis. 2019, 18 (1), 1-10. 
(5) Yamashita, S.; Hashimoto, M.; Haque, A. M.; Nakagawa, K.; Kinoshita, M.;

Shido, O.; Miyazawa, T. Lipids 2017, 52 (7), 575-585.

(6) Rustam, Y. H.; Reid, G. E. Anal. Chem. 2018, 90 (1), 374-397.

(7) Otoki, Y.; Kato, S.; Kimura, F.; Furukawa, K.; Yamashita, S.; Arai, H.; Miyazawa, T.; Nakagawa, K. J. Pharm. Biomed. Anal. 2017, 134, 77-85.

(8) Otoki, Y.; Nakagawa, K.; Kato, S.; Miyazawa, T. J. Chromatogr. B Anal. Technol. Biomed. Life Sci. 2015, 1004, 85-92.

(9) Murphy, E. J.; Stephens, R.; Jurkowitz-Alexander, M.; Horrocks, L. A. Lipids 1993, $28(6), 565-568$.

(10) Fhaner, C. J.; Liu, S.; Ji, H.; Simpson, R. J.; Reid, G. E. Anal. Chem. 2012, 84 (21), 8917-8926.

(11) Rapport, M. M.; Lerner, B. BBA - Biochim. Biophys. Acta 1959, 33 (2), 319-325.

(12) Fhaner, C. J.; Liu, S.; Zhou, X.; Reid, G. E. Mass Spectrom. 2013, 2 (Special_Issue), S0015-S0015.

(13) Canez, C. R.; Shields, S. W. J.; Bugno, M.; Wasslen, K. V.; Weinert, H. P.; Willmore, W. G.; Manthorpe, J. M.; Smith, J. C. Anal. Chem. 2016, 88 (14), 69967004.

(14) Wasslen, K. V.; Canez, C. R.; Lee, H.; Manthorpe, J. M.; Smith, J. C. Anal. Chem. 2014, 86 (19), 9523-9532.

(15) Shields, S. W. J.; Manthorpe, J. M. J. Label. Compd. Radiopharm. 2014, 57 (12), 674-679.

(16) Pluskal, T.; Castillo, S.; Villar-Briones, A.; Orešič, M. BMC Bioinformatics 2010, 11. 
(17) Korf, A.; Jeck, V.; Schmid, R.; Helmer, P. O.; Hayen, H. Anal. Chem. 2019, 91 (8), 5098-5105.

(18) Sud, M.; Fahy, E.; Cotter, D.; Brown, A.; Dennis, E. A.; Glass, C. K.; Merrill, A. H.; Murphy, R. C.; Raetz, C. R. H.; Russell, D. W.; et al. Nucleic Acids Res. 2007, 35 (SUPPL. 1), 527-532.

(19) Lerno, L. A.; German, J. B.; Lebrilla, C. B. Anal. Chem. 2010, 82 (10), 42364245.

\subsection{Supporting Information}

Table 3.7.1 (S1) Full list of UNMOD plasmalogens identified in BLE that have greater than $\mathbf{5 0 \%}$ decrease in peak area after formic acid treatment

\begin{tabular}{ccccc}
\hline m/z & $\begin{array}{c}\text { Retention } \\
\text { Time (min.) }\end{array}$ & $\begin{array}{c}\text { Average } \\
\text { Normalized } \\
\text { Peak Area }\end{array}$ & $\begin{array}{c}\text { Standard } \\
\text { error (\%) }\end{array}$ & $\begin{array}{c}\text { Decrease in } \\
\text { Peak } \\
\text { Area after Acid } \\
\text { Treatment (\%) }\end{array}$ \\
\hline 686.5103 & 10.64 & 0.22 & 1.0 & 86.5 \\
698.5104 & 10.56 & 0.26 & 3.3 & 87.1 \\
700.5263 & 11.74 & 0.90 & 2.6 & 85.1 \\
702.5414 & 13.02 & 0.34 & 3.7 & 87.0 \\
704.5572 & 11.26 & 0.38 & 2.0 & 88.9 \\
714.5416 & 9.98 & 0.75 & 3.4 & 85.8 \\
716.5571 & 10.92 & 0.87 & 3.4 & 86.5 \\
718.5724 & 12.46 & 0.25 & 3.8 & 92.3 \\
728.5574 & 11.57 & 1.89 & 7.9 & 75.4 \\
730.5729 & 11.60 & 0.84 & 2.2 & 86.9 \\
738.5415 & 9.88 & 0.50 & 5.6 & 81.7 \\
740.5591 & 10.46 & 0.98 & 2.7 & 83.8 \\
742.5729 & 11.61 & 2.32 & 2.6 & 85.8 \\
744.5862 & 12.33 & 1.47 & 2.1 & 80.3 \\
748.5252 & 11.41 & 0.26 & 2.1 & 88.2 \\
750.5442 & 11.97 & 0.52 & 3.9 & 81.4
\end{tabular}




$\begin{array}{lcccc}752.5571 & 13.23 & 0.91 & 3.8 & 49.9 \\ 752.5571 & 10.54 & 1.13 & 5.3 & 87.3 \\ 756.5864 & 12.25 & 0.22 & 15.4 & 85.5 \\ 764.5570 & 10.33 & 0.83 & 1.9 & 84.8 \\ 766.5727 & 11.22 & 2.16 & 2.8 & 85.5 \\ 768.5882 & 11.89 & 0.69 & 6.1 & 63.8 \\ 770.6022 & 13.43 & 0.28 & 5.1 & 88.3 \\ 776.5572 & 10.26 & 0.42 & 3.3 & 87.7 \\ 778.5725 & 10.86 & 0.87 & 4.6 & 84.7 \\ 780.5884 & 11.84 & 0.89 & 5.2 & 82.8 \\ 790.5726 & 11.29 & 0.74 & 4.1 & 85.6 \\ 792.5880 & 12.09 & 1.90 & 4.0 & 83.6 \\ 794.6031 & 13.16 & 1.36 & 4.0 & 82.7\end{array}$

Table 3.7.2 (S2) List of TrEnDi modified plasmalogens in BLE

\begin{tabular}{|c|c|c|c|c|}
\hline $\mathrm{m} / \mathrm{z}$ & $\begin{array}{l}\text { Retention } \\
\text { Time } \\
\text { (min.) }\end{array}$ & $\begin{array}{c}\text { Average } \\
\text { Normalized } \\
\text { Peak Area }\end{array}$ & $\begin{array}{l}\text { Standard } \\
\text { error }(\%)\end{array}$ & $\begin{array}{c}\text { Decrease } \\
\text { in Peak } \\
\text { Area after } \\
\text { Acid } \\
\text { Treatment } \\
(\%)\end{array}$ \\
\hline 719.5784 & 10.17 & 1.07 & 2.3 & 99.4 \\
\hline 729.5619 & 9.34 & 3.08 & 3.5 & 99.9 \\
\hline 731.5797 & 9.86 & 2.36 & 3.3 & 98.0 \\
\hline 732.5763 & 9.32 & 10.08 & 6.1 & 99.2 \\
\hline 733.5922 & 11.00 & 0.44 & 0.7 & 98.8 \\
\hline 734.5844 & 9.95 & 4.13 & 4.8 & 98.2 \\
\hline 743.5775 & 9.62 & 4.68 & 2.1 & 99.9 \\
\hline 745.5937 & 10.32 & 2.08 & 1.9 & 99.6 \\
\hline 746.5878 & 9.60 & 7.72 & 11.0 & 99.7 \\
\hline 753.5649 & 9.14 & 2.58 & 3.5 & 99.4 \\
\hline 755.5778 & 9.51 & 3.37 & 2.6 & 99.0 \\
\hline 757.5938 & 10.42 & 6.00 & 1.8 & 99.8 \\
\hline 758.5854 & 9.44 & 10.08 & 4.9 & 99.1 \\
\hline 759.6093 & 11.50 & 2.38 & 4.4 & 99.9 \\
\hline 760.6044 & 10.27 & 23.92 & 3.5 & 99.5 \\
\hline 762.6211 & 11.62 & 6.38 & 5.0 & 98.8 \\
\hline 767.5778 & 9.54 & 4.31 & 3.3 & 99.9 \\
\hline 771.6074 & 10.80 & 0.55 & 3.4 & 99.9 \\
\hline 779.5776 & 9.38 & 2.74 & 10.2 & 98.1 \\
\hline 781.5937 & 10.00 & 5.15 & 1.6 & 98.9 \\
\hline
\end{tabular}




$\begin{array}{lcccc}783.6091 & 10.77 & 1.82 & 2.8 & 89.3 \\ 785.6262 & 12.22 & 0.92 & 17.6 & 99.7 \\ 791.5779 & 9.45 & 2.02 & 2.4 & 99.9 \\ 793.5937 & 9.81 & 2.69 & 1.5 & 97.6 \\ 795.6090 & 10.43 & 2.06 & 8.1 & 98.9 \\ 805.5929 & 10.17 & 2.07 & 2.6 & 100.0 \\ 807.6092 & 10.56 & 5.31 & 2.4 & 99.1 \\ 808.6061 & 10.14 & 6.56 & 3.0 & 99.7 \\ 809.6247 & 11.43 & 3.49 & 1.8 & 99.7 \\ 810.6218 & 10.71 & 18.33 & 4.4 & 95.9 \\ 812.6366 & 11.51 & 22.51 & 7.7 & 78.2\end{array}$

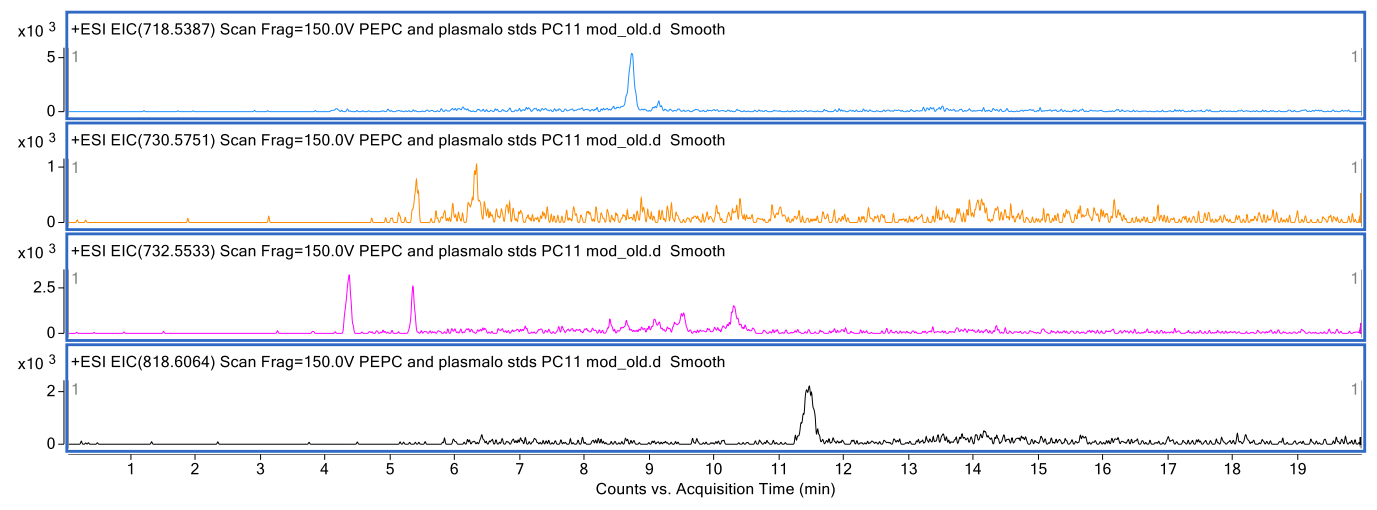

Figure 3.7.1 (S1) Unmodified lipid standards left after the reaction with DZM using non-optimized conditions 


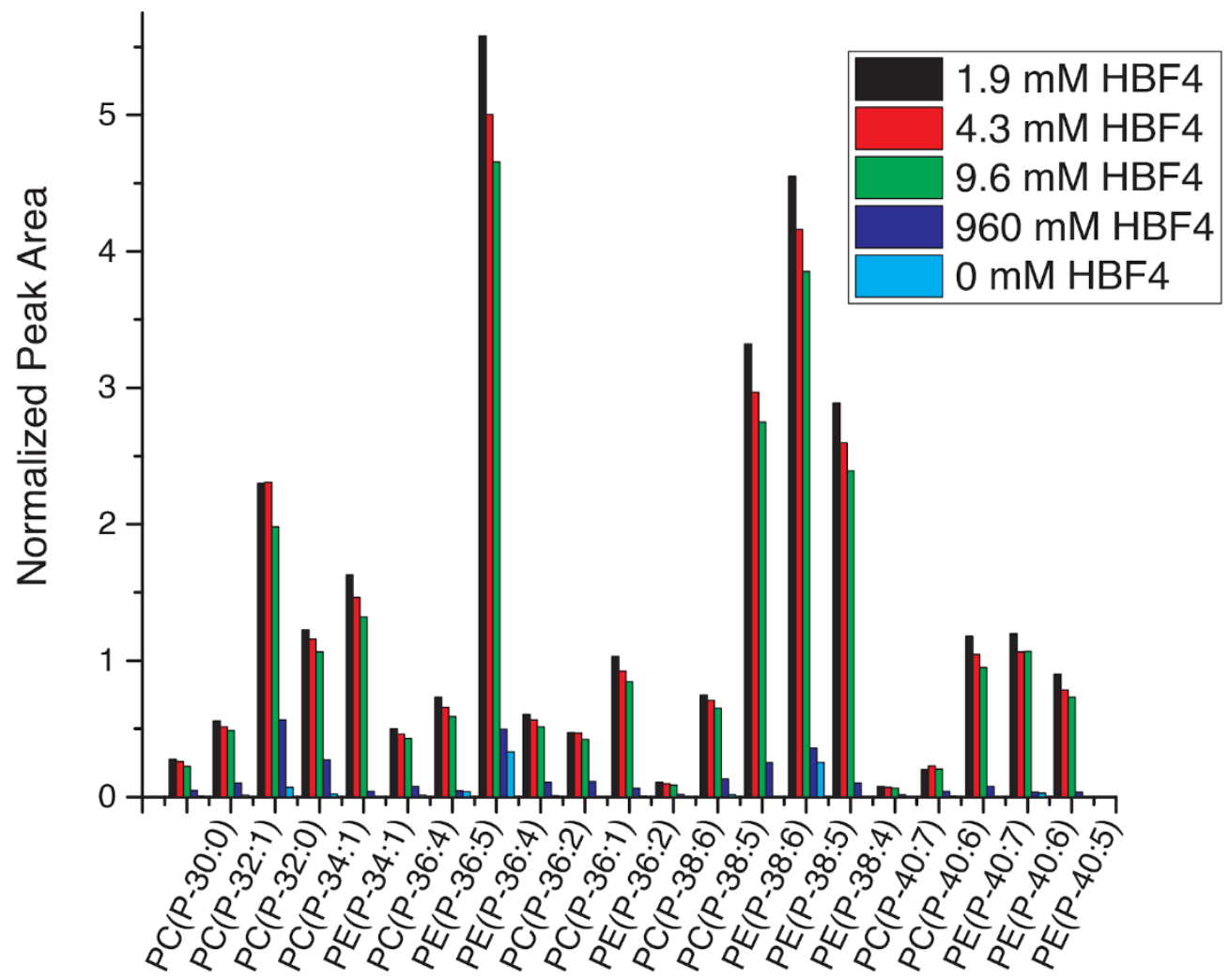

Bulk Lipid Names

Figure 3.7.2 (S2) Acid concentration optimization on HeLa extract showing how

$\left[\mathrm{H}^{+}\right]$changes the abundance of fully modified plasmalogens

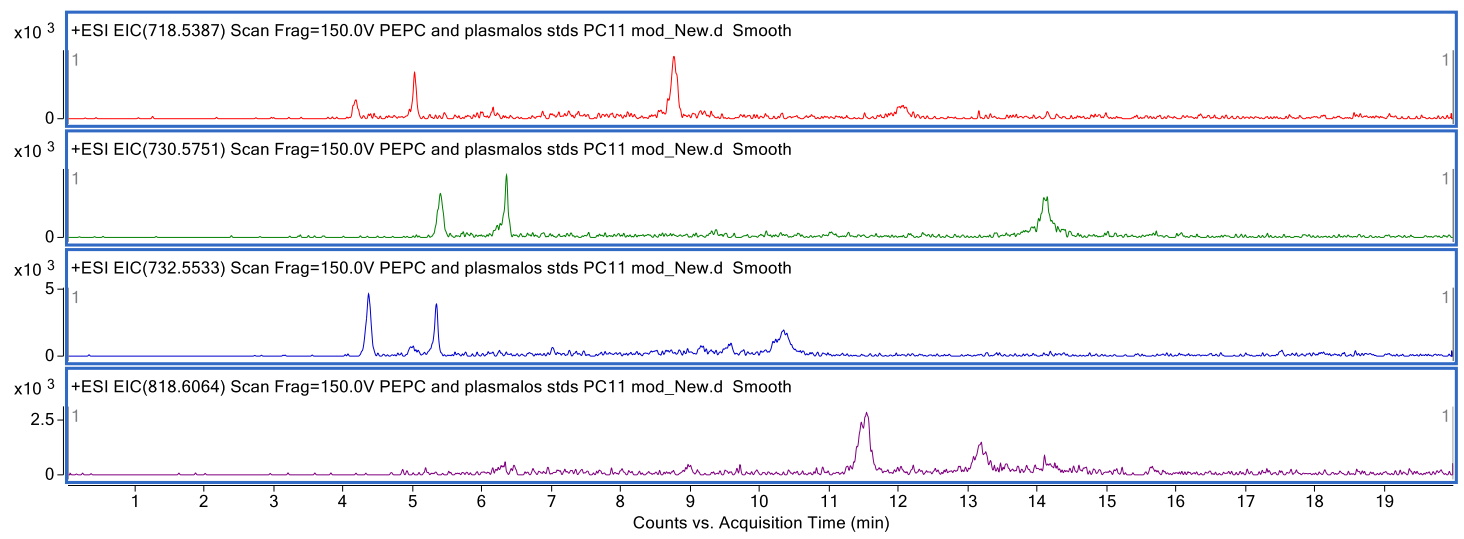

Figure 3.7.3 (S3) Unmodified lipid standards left after the reaction with DZM using optimized conditions 


\section{Chapter 4: Improved Analysis of Glycerphospholipids via a Micro- scale In Situ Derivatization Strategy using Diazoalkanes}

Samuel W. J. Shields ${ }^{\mathrm{a}, \mathrm{c}}$, Christian A. Rosales ${ }^{\mathrm{a}, \mathrm{c}}$, Peter J. Pallister ${ }^{\mathrm{a}, \mathrm{c}}$, Joshua A. Roberts ${ }^{\mathrm{b}, \mathrm{c}}$, Karl V. Wasslen ${ }^{\mathrm{a}, \mathrm{c}}$, Jeffrey M. Manthorpe ${ }^{\mathrm{a}, \mathrm{b}, \mathrm{c} *}$, Jeffrey C. Smith $^{\mathrm{a}, \mathrm{b}, \mathrm{c} *}$

a) Department of Chemistry Carleton University, Ottawa, Ontario K1S 5B6, Canada

b) Institute of Biochemistry, Carleton University, Ottawa, Ontario K1S 5B6, Canada

c) Carleton Mass Spectrometry Centre, Carleton University, Ottawa, Ontario K1S 5B6, Canada

* to whom correspondence should be addressed - jeff.smith@carleton.ca, jeffrey.manthorpe@,carleton.ca

Keywords: lipids, glycerophospholipids, diazomethane, diazoethane, mass spectrometry

\subsection{Abstract}

Using Trimethylation Enhancement using Diazomethane (TrEnDi), our lab has shown significant enhancements to the signal intensity of glycerophospholipid species in MS/MS analyses. Here we describe a novel apparatus and derivatization methodology, termed in situ TrEnDi (iTrEnDi), for the preparation of small amounts of gaseous diazoalkanes in situ. iTrEnDi allows clean and rapid reactions of phosphatidylcholine (PC), phosphatidylethanolamine (PE), phosphatidic acid (PA) and sphingomyelin (SM) with no direct handling of dangerous diazoalkane solutions. Methylated PC and PE showed similar sensitivity enhancements and fragmentation patterns using the iTrEnDi apparatus compared to our previously reported methodology. The methylation of PA resulted in 
primarily a dimethylated PA and exhibited a dramatic improvement in chromatographic resolution and 14x fold increase in sensitivity when analyzed via LCMS. Diazoethane, a more reactive diazoalkane, was also prepared with iTrEnDi and reacted with PC and SM that resulted in a characteristic fragment of $\mathrm{m} / \mathrm{z} 212.1$ when the products were analyzed by MS/MS. A comparison of solution based TrEnDi to iTrEnDi was made that resulted in a modest decrease in sensitivity likely due to analyte losses during handling. However, the benefits of using a much safer technique combined with iTrEnDi's ability to accommodate more reactive diazoalkanes, vastly improves the access and flexibility of the method.

\subsection{Introduction}

Lipidomics, the study of lipids and their dynamics in living systems, has become more prominent in literature due in part to the propensity of complex lipid samples to be analyzed by mass spectrometry (MS). Over the past several years, many reports have emerged in the field of lipid analysis, detailing novel roles for lipids in cellular signaling in both healthy cells and disease processes,${ }^{1-3}$ resulting in a plethora of analytical-based studies on lipids in literature. ${ }^{4,5}$ Lipids comprise a diverse class of biomolecules with a range of physicochemical properties. Glycerophospholipids form the basis of cellular membranes, are amphipathic in nature and vary in the extent to which they may be observed via MS. ${ }^{6}$ For example, phosphatidylcholine (PC) contains a zwitterionic head group (above $\mathrm{pH} 1)^{7}$ and may be easily ionized in positive ion mode using electrospray ionization (ESI), whereas phosphatidic acid (PA) prefers to exists as an anion in both the solution and gas phase. ${ }^{8}$ This translates to challenges in MS-based lipidomic studies, where different polarities or solvent conditions are required for optimal analysis of different 
glycerophospholipid species. Using a single method introduces bias against certain glycerophospholipid subclasses; the analyst is faced with either using multiple separation methods and consuming greater amounts of sample, or more commonly, limiting the scope of their study to the analysis of a selection of glycerophospholipid subclasses. Chemical derivatization strategies have emerged over the past years to address the analytical heterogeneity of glycerophospholipids and improve MS-based lipidomic datasets. Lipid derivatization as a sample preparation technique is a highly valuable tool when analyzing complex lipid samples to increase sensitivity, resolve isobaric species and enhance structural information. ${ }^{9}$ Many strategies have been employed over the last two decades to install a readily ionizable group on a variety of lipid structures. Examples include reactions with N-hydroxysuccinimide (NHS) esters to install a trialkyl sulfonium moiety on phosphatidylethanolamine lipids (PE),${ }^{10}$ preparation of esters via Mitsunobu reaction ${ }^{11}$ of diacylglycerol (DAG) species, and methylation of phosphatidylglycerol (PG) lipids. ${ }^{12}$

Chemical derivatization techniques in lipidomics demand that any reaction must be rapid, have high conversion rates, afford minimal side products that do not interfere with MS analysis as well as function on a very small scale (>10 nmol). Diazomethane (DZM) is a reagent that has been implemented in the gas chromatography MS analysis of fatty acids for many years ${ }^{13}$ due to its ability to alkylate free fatty acids cleanly. Diazoalkanes are highly reactive reagents, also reacting with other acidic functional groups to produce tetraalkylammonium species, methyl esters and alkyl phosphates with $\mathrm{N}_{2}(\mathrm{~g})$ being the sole by-product produced. Regrettably, diazoalkanes (especially DZM and diazoethane) are hazardous to handle due to their highly toxic and explosive nature. ${ }^{14}$ Furthermore, they must be prepared with caution from a precursor that has shipping restrictions across 
international borders. Although trimethylsilyl DZM is a safe to handle, commercially available derivatization agent, it lacks the reactivity necessary for complete methylation of glycerophospholipids. We have developed a solution phase chemical derivatization approach using a solution of DZM in ether for the methylation of tryptic peptides and glycerophospholipids. ${ }^{15-17}$ With the use of ${ }^{13} \mathrm{C}-\mathrm{DZM}$, prepared inexpensively from ${ }^{13} \mathrm{C}$ Diazald, ${ }^{18}$ the average sensitivity increase gained from the TrEnDi protocol and tandem MS analysis on average was 11 fold and 3 fold for PE and PC phospholipid classes from HeLa cell extract, respectively. The in situ preparation of DZM has been described previously ${ }^{19-22}$ where large (1.5 g of precursor) amounts of DZM were generated and used immediately without distillation in the gaseous form. Herein, we describe a novel apparatus to safely prepare small $(0.1 \mathrm{~g}$ precursor) amounts of diazoalkanes in situ without direct handling of the diazoalkane solution for the micro-scale derivatization of PC, PE, PA and SM.

\subsection{Experimental:}

Chemicals and Materials. Ammonium acetate, $\mathrm{HBF}_{4}-\mathrm{Et}_{2} \mathrm{O}$ was purchased from SigmaAldrich (St. Louis, MO, USA); LCMS grade isopropanol, methanol, and water was purchased from Fisher scientific (Hampton, NH, USA); PE(16:0/18:1(9Z)), PC(16:0/18:1(9Z)), and PC(11:0/11:0) were obtained from Avanti Polar Lipids Inc. (Alabaster, Alabama, USA); $\mathrm{KOH}$ and $\mathrm{Et}_{2} \mathrm{O}$ were purchased from Caledon Laboratories Ltd. (Georgetown, ON, Canada). A full list of materials used to construct iTrEnDi apparatus can be found in the supplementary information (Pg. 8). $N$-methyl- $N$-nitrosourea and $N$-ethyl- $N$-nitrosourea were readily prepared via previously reported methods. ${ }^{23}$ 


\section{Diazoalkane Preparation and Simultaneous Phospholipids Derivatization. CAUTION:}

All reactions involving preparation and derivatization with diazoalkanes should be performed in a chemical fume hood with efficient ventilation and behind a polycarbonate blast shield due to their toxic and explosive nature. For a complete visual representation of the experimental setup, refer to Figure 1. A solution of aqueous $\mathrm{KOH}(1 \mathrm{ml}, 6.1 \mathrm{M})$ was added into a sealed headspace vial dropwise (1 drop/s) into a mixture of $N$-alkyl- $N$ nitrosourea (100 mg, $0.8 \mathrm{mmol}), \mathrm{H}_{2} \mathrm{O}(2 \mathrm{ml})$, and $\mathrm{Et}_{2} \mathrm{O}(1.4 \mathrm{ml})$ at room temperature. The diazoalkane/ $\mathrm{N}_{2}$ output line of the reactor was inserted into a methanol solution containing glycerophospholipid $(100 \mu \mathrm{L}, 25 \mu \mathrm{M})$ and $\mathrm{HBF}_{4}-\mathrm{Et}_{2} \mathrm{O}(1.0 \mu \mathrm{mol})$. Upon the addition of aqueous $\mathrm{KOH}$, vigorous effervescence was observed (the rate of addition should be adjusted to minimize frothing). After the addition of base was complete, a biphasic system was obtained, where ethereal diazoalkane (yellow color) resided in the top layer and the $\mathrm{N}_{2}$ (in) gas line was adjusted to be submerged under the ether layer for the remainder of the reaction time. The reaction was allowed to proceed at room temperature until minimal yellow color was observed in the reactor $(\sim 1 \mathrm{~h})$. Residual solvent was evaporated via a stream of $\mathrm{N}_{2}(\mathrm{~g})$ and stored at $-20^{\circ} \mathrm{C}$. Alkylated lipid samples were resuspended in $\mathrm{MeOH}$ to $1 \mu \mathrm{M}$ each with an external standard PC (11:0/11:0) $(1 \mu \mathrm{M})$.

LCMS Analysis. An $8 \mu \mathrm{L}$ aliquot of lipid sample was injected using auto sampler of an Agilent 1290 infinity system and separated with an Agilent Poroshell 120 column (EC-C18 $2.7 \mu \mathrm{m}, 2.1 \times 100 \mathrm{~mm}$ ). The column was maintained at $40{ }^{\circ} \mathrm{C}$ and a variable flow rate of 0.21-0.28 mL/min. The mobile phases consisted of (A) 50:50 (v/v) methanol:water with ammonium acetate $(10 \mathrm{mM})$ and (B) 75:50 (v/v) methanol:isopropanol with ammonium acetate $(10 \mathrm{mM})$. The separation gradient was 0 min $0 \%(B) ; 0.5 \min 15 \%(B) ; 2.5 \mathrm{~min}$ 
$30 \%$ (B); $3.0 \min 50 \%$ (B); $5-18 \min 100 \%$ (B); $19-24 \min 0 \%$ (B). The eluent was introduced into an Agilent 6550 iFunnel using the parameters: ESI polarity positive; MS, $\mathrm{m} / \mathrm{z} 100-1700$; capillary voltage, $4 \mathrm{kV}$; nozzle voltage, $3 \mathrm{kV}$; gas temperature, $275^{\circ} \mathrm{C}$; drying gas, $12 \mathrm{~L} / \mathrm{min}$; nebulizer gas, $37 \mathrm{psig}$; sheath gas temperature, $300{ }^{\circ} \mathrm{C}$; sheath gas flow, 6.5 L/min; fragmentor, $150 \mathrm{~V}$; collision energy, $30 \mathrm{eV}$ and tuned using an Agilent tune mix.

\subsection{Results and Discussion}

We have recently reported the use of DZM prepared in multi milliliter batches via a co-distillation process for the conversion of neutral phospholipids to a fixed positive charge tetraalkylammonium species. DZM can also be prepared via a mixture of $\mathrm{KOH}(\mathrm{aq})$, $\mathrm{Et}_{2} \mathrm{O}$ and $N$-nitroso- $N$-methyl urea (NMU), where ethereal DZM can be decanted, and stored for a week. In our experience, the DZM produced from NMU is heavily contaminated with reaction by-products, such as $\mathrm{KOCN}$, and unreacted starting material (Figure S1). Only individuals with extensive training are permitted to prepare DZM in batch-scale synthesis in solution or by distillation in order to reduce associated health and safety risks.

Considering the need to avoid batch distillation due to safety concerns and avoiding the contamination from the DZM produced from NMU, the best characteristics of the two methods were combined. This allows for a clean gaseous reagent and the use of the more reactive precursor, NMU, that readily converts to DZM at or below room temperature. The result was a small scale, in situ method of derivatizing phospholipids with a continuous flow of gaseous DZM that was safely carried with $\mathrm{Et}_{2} \mathrm{O}$ vapour from the reaction vessel. 
The benefits of using an in situ approach includes eliminating the need for DZM to be stored in large quantities and initiating the reaction remotely through the addition of $\mathrm{KOH}_{(\mathrm{aq})}$ via syringe pump. Furthermore, the DZM is prepared fresh for each derivatization, leading to greater reproducibility between reactions of the same analyte.

The apparatus used for the methylation of phospholipids can be constructed from readily available inexpensive laboratory materials as depicted in Figure 4.4.1 (see table S1 for list of materials used). This modular device is assembled using two crimp-top glass vials, a syringe and a nitrogen source connected by fused silica tubing. The diazo generating vial contains an aqueous mixture of precursor and diethylether, which is capped with a septum. The vial's septum is then pierced by three lines of fused silica; the first is submerged into the aqueous mixture and delivers 10 psi of nitrogen and the second is used to administer aqueous $\mathrm{KOH}$ at a controlled rate. The third line, suspended above the reaction mixture, delivers a constant stream of in situ generated diazoalkane carried by ether vapor to a small sample vial containing analyte(s). This apparatus for in situ diazoalkane generation and the associated TrEnDi derivatizations of analytes have been termed, in situ TrEnDi (iTrEnDi). 


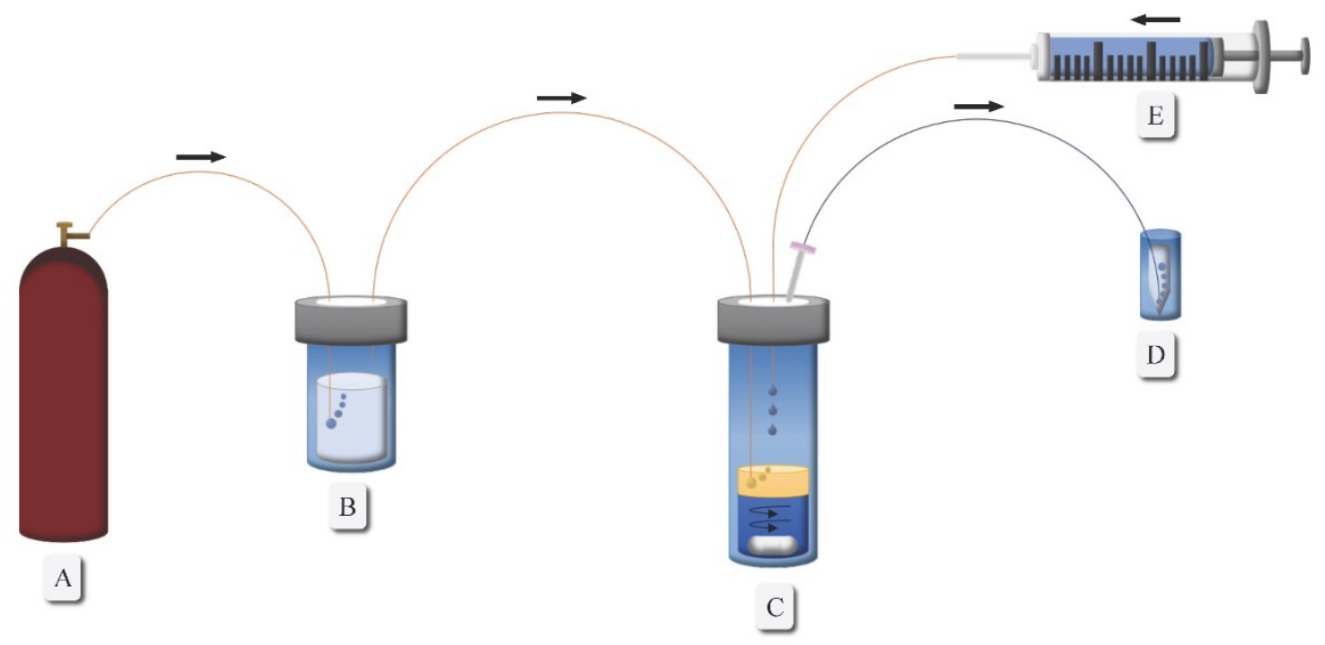

Figure 4.4.1 Diagram of the experimental setup of iTrEnDi derivatization method.
A) $\mathrm{N}_{2}$ gas source. B) diethyl ether- $\mathrm{N}_{2}$ formation vial. C) Diazoalkane reaction
chamber ( $\mathrm{H}_{2} \mathrm{O}, \mathrm{NMU}$, diethyl ether) D) Analyte vial
(glycerophospholipids/sphingolipids) E) Base solution (6.1 $\mathrm{M} \mathrm{KOH}$ in $\mathrm{H}_{2} \mathrm{O}$ )

Initially, three phospholipid standards were reacted with DZM using iTrEnDi (Figure 4.4.2). Phosphatidylethanolamine (PE 16:0/18:1) and phosphatidylcholine (PC 19:0/19:0) were derivatized as an equimolar mixture. The reactions of PC and PE resulted in the addition of one $\mathrm{CH}_{2}(14 \mathrm{Da})$ and four $\mathrm{CH}_{2}(56 \mathrm{Da})$ units respectively when analyzed via LCMS. Following the reaction, it was observed that under 3\% unmodified PC and less than $1 \%$ unmodified PE remained. The combined peak area of the lower methylation states of PE was also observed to be less than $1 \%$ indicating that the reaction of PE with DZM was complete (Figure S2). Tandem MS analysis of the derivatized lipids resulted in fragmentation to methyl-phosphocholine (m/z 198) and a neutral loss of $\mathrm{NMe}_{3}$ from the head group (m/z 139). 
Derivatization of sphingomyelin (SM d18:1/16:0) via iTrEnDi resulted in the addition of one $\mathrm{CH}_{2}(14 \mathrm{Da})$ and two $\mathrm{CH}_{2}(28 \mathrm{Da})$ groups. The reaction of SM d18:1/16:0 resulted in less than $2 \%$ unmodified lipid product as well as a product bearing a mass shift consistent with the addition of two $\mathrm{CH}_{2}$ groups that had an $11 \%$ abundance relative to the singly methylated product. Tandem MS analysis of the monoalkylated and dialkylated SM species resulted in similar fragment ions as PC 19:0/19:0. Upon closer inspection of the tandem MS spectrum of dialkylated SM, a small fragment ( $\mathrm{m} / \mathrm{z} 534)$ was observed and is consistent with additional alkylation on the ceramide backbone. We hypothesize that either the amide $\mathrm{NH}$ or allylic alcohol $\mathrm{OH}$ could have a low enough $\mathrm{pKa}$ to be partially methylated with DZM.
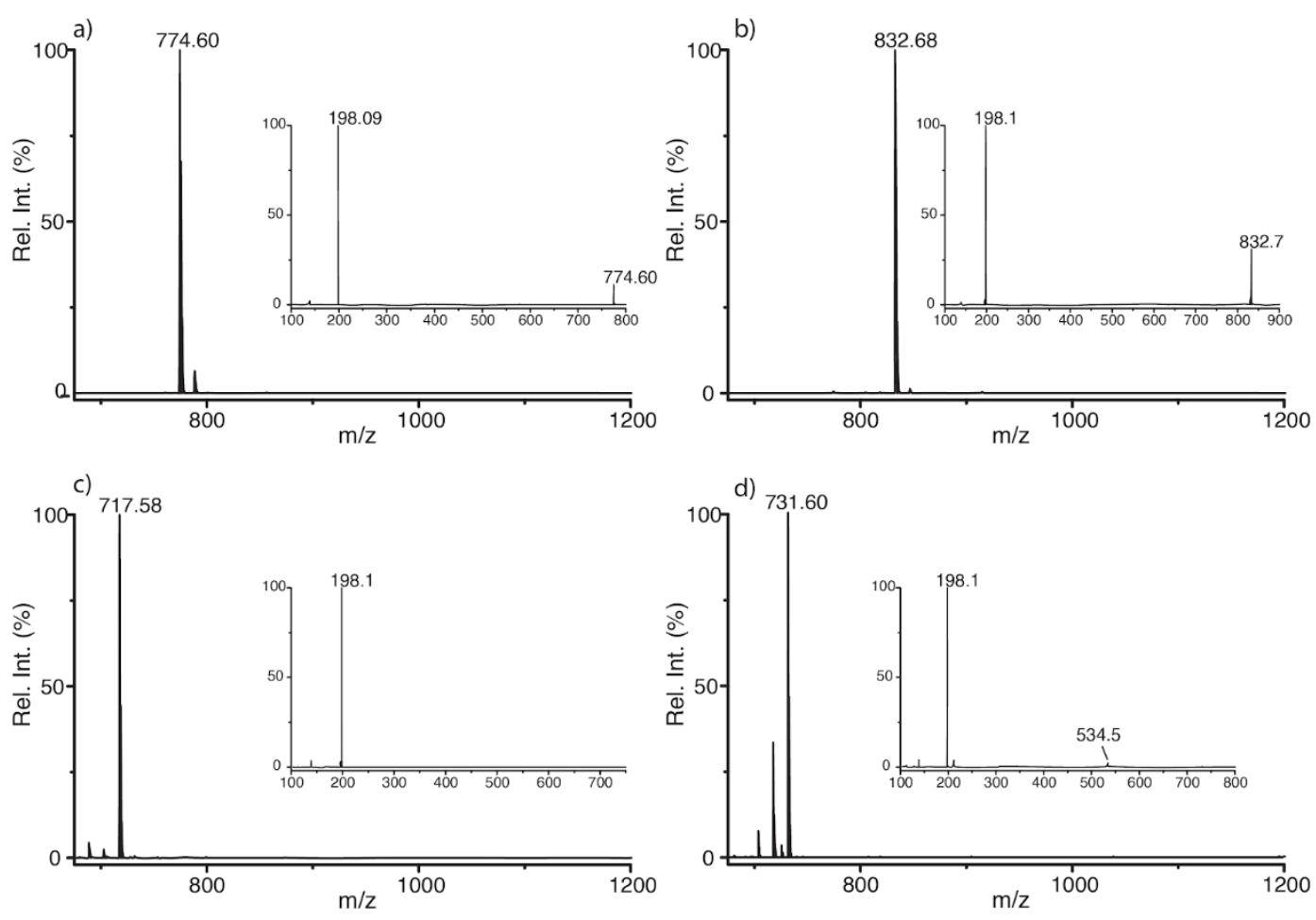

Figure 4.4.2 LCMS and MS² of derivatized lipid standards via iTrEnDi in situ methylation with DZM. a) PE 16:0/18:1. b) PC 19:0/19:0. c) SM d18:1/16:0. d) Over methylation product of SM d18:1/16:0. 
Phosphatidic acids are a highly amphipathic lipid species that can be readily ionized in negative ion mode as $[\mathrm{M}-\mathrm{H}]^{-}$or in positive ion mode as $\left[\mathrm{M}+\mathrm{NH}_{4}\right]^{+}$. The liquid chromatography (LC) MS analysis of PA lipids are known to be challenging since very broad elution profiles are observed when using reverse phase chromatography. ${ }^{24,25}$ Generally many of the changes in chromatographic conditions to enhance the analysis of PA analysis are not amenable to all other classes of glycerophospholipids. The poor elution profiles in combination with PA lipid species being observed in very low abundance in biological systems make them a challenging analyte for LCMS analyses. ${ }^{26}$

Prior to derivatization with traditional lipidomic LCMS conditions, we found that PA 12:0/12:0 in positive ion mode showed an ion distribution based on normalized peak areas of $[\mathrm{M}+\mathrm{NH} 4]^{+}(100 \%),[\mathrm{M}+\mathrm{Na}]^{+}(77 \%),[\mathrm{M}+\mathrm{H}]^{+}(4 \%)$ and a $\left[\mathrm{M}-\mathrm{NH}_{4}{ }^{+} \mathrm{PO}_{4} \mathrm{H}_{2}\right]^{+}(22 \%)$ consistent with the insource loss of PA headgroup. When comparing peak areas, the ammoniated PA was significantly larger than deprotonated product in negative ion mode (Figure 4.4.3a). The reaction of phosphatidic acid (PA 12:0/12:0) with gaseous DZM resulted in mostly dimethylated material (28 Da), a small amount of singly methylated material (less than 1\%), and no observable remaining unreacted lipid. The distribution of ions based on normalized peak areas formed with dimethyl PA 12:0/12:0 is as follows, $\left[\mathrm{M}+\mathrm{NH}_{4}\right]^{+}(100 \%),[\mathrm{M}+\mathrm{Na}]^{+}(12 \%),[\mathrm{M}+\mathrm{H}]^{+}(7 \%)$ and a $\left[\mathrm{M}-\mathrm{NH}_{4}{ }^{+} \mathrm{PO} 4 \mathrm{H}_{2}\left(\mathrm{CH}_{3}\right)_{2}{ }^{-}\right]^{+}(41 \%)$. Interestingly, the methylation of PA has reduced signal splitting of PA to mostly ammoniated adduct, despite increasing the insource headgroup loss. Overall, normalized peak areas resulted in the in the 13.9-fold increase of dimethyl PA over unmodified PA. 
Unlike the other lipid species discussed above, the methylation of PA 12:0/12:0 yields a neutral molecule rather than a lipid bearing a fixed positive charge that gives rise to multiple adducts formed in source. This signal splitting and in source fragmentation can make PA analysis in complex samples challenging; however, following iTrEnDi derivatization, we observed a substantial increase in signal peak area and chromatographic resolution (Figure 3b). Even with polar protic solvents in our chromatography eluents, we propose that the decrease in hydrogen bonding of the dimethylated phosphoric acid produces a more favorable interaction with the solid phase of the C18 column. Finally, the tandem MS analysis of dimethyl PA 12:0/12:0 $\left[\mathrm{M}+\mathrm{NH}_{4}\right]^{+}$yielded the neutral loss of the phosphate head group, and in fatty acyl chain loss as acylium ions (m/z 183). The novel increase in chromatographic resolution, sensitivity increase as well as the fragmentation of $\left[\mathrm{PA}^{\mathrm{Tr}}+\mathrm{NH}_{4}\right]^{+}$to give fatty acyl chain information renders the iTrEnDi methodology for analysis of PA lipids is very promising for future PA analyses via LCMS. 

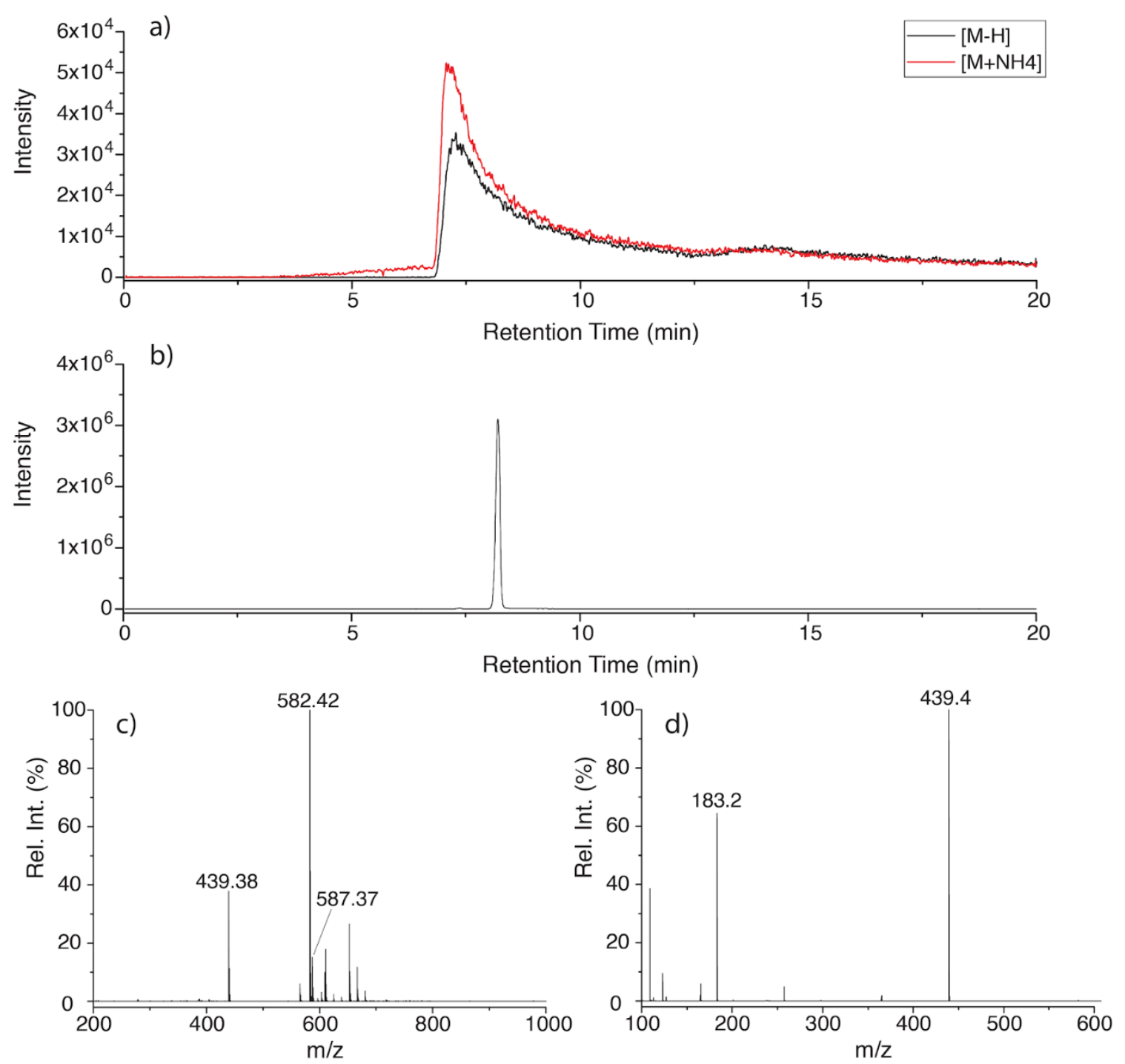

Figure 4.4.3 a) XIC of m/z 554.38 for underivatized PA 12:0/12:0 ammonium adduct. b) XIC of 582.41 for $\mathrm{PA}^{\mathrm{Tr}}$ 12:0/12:0 ammonium adduct. c) MS showing iTrEnDi-modified PA 12:0/12:0 with both ammoniated and sodiated adducts. d) $\mathrm{MS}^{2}$ of the ammoniated adduct of $\mathrm{PA}^{\mathrm{Tr}}$ 12:0/12:0.

To assess our novel method of DZM derivatization of phospholipids, a side-by-side comparison of the in solution TrEnDi and iTrEnDi protocols was performed on PE and PC standards (Figure 4.4.4). The experiments consisted of performing both protocols in 
chemical triplicates with PC 11:0/11:0 as an external standard. After normalization of peak areas, the external standard showed a discrepancy between the two methods. In general, both protocols yielded an increase in sensitivity compared to unmodified lipids, but the gains for the in solution TrEnDi method had modest improvements over iTrEnDi method. The lower signal could be attributed to loss of the analyte through degradation due to longer reaction times and the greater extent to which the samples are handled when undergoing iTrEnDi modification. Although a modest decrease in sensitivity is observed with lipid analytes when using iTrEnDi methodology, we have expanded to a simpler, user-friendly protocol, granting indirect handling of toxic and explosive diazoalkanes whilst enabling the production of diazoalkanes on a need-to-use basis.

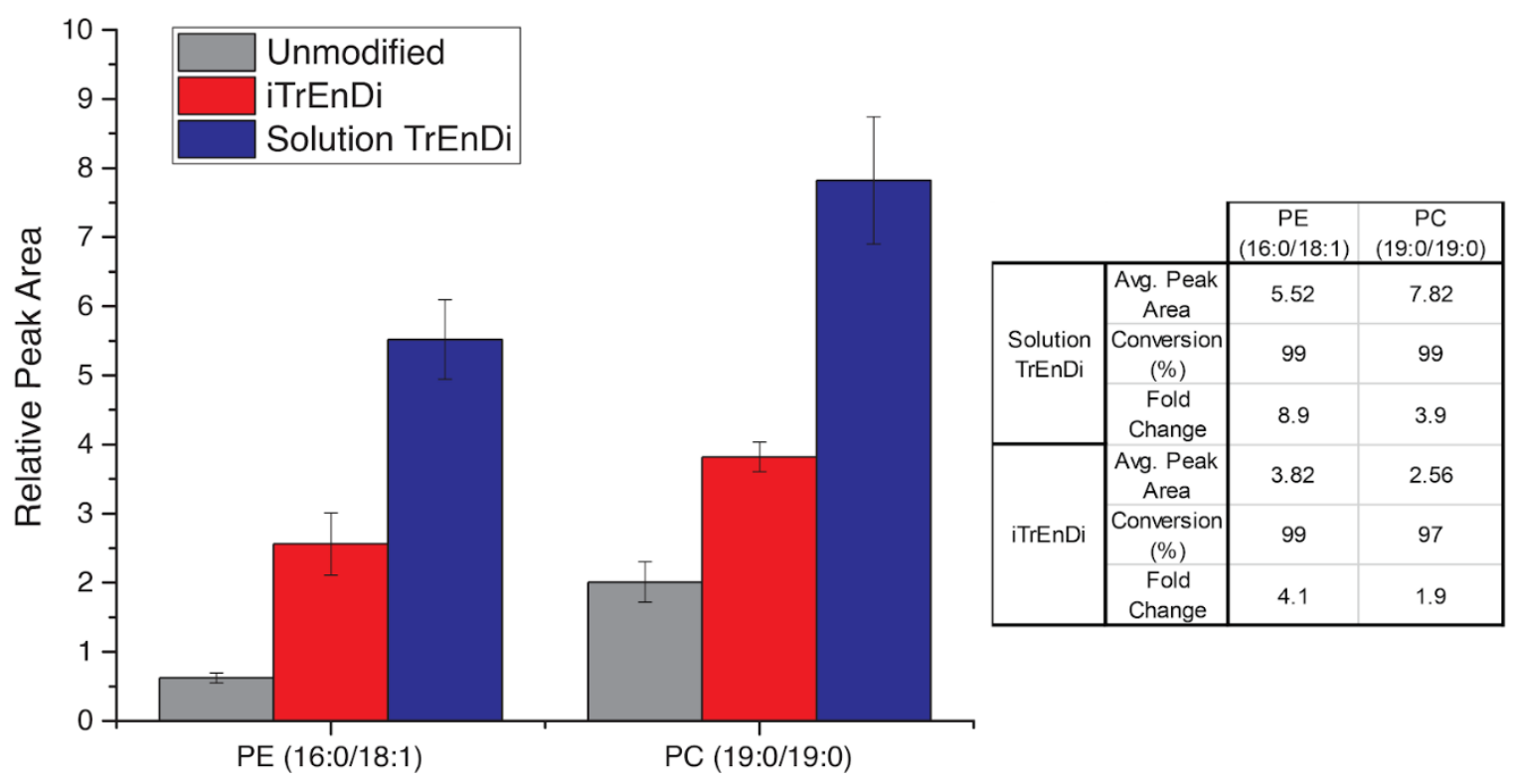

Figure 4.4.4 Comparison of in-solution TrEnDi vs. iTrEnDi using chemical triplicates to unmodified lipid standards

This novel apparatus can also accommodate the preparation of other reactive diazoalkanes. We readily prepared $N$-ethyl- $N$-nitrosourea (NEU), and used it in the 
iTrEnDi apparatus to safely synthesize diazoethane in situ. The reaction of diazoethane with PC 16:0/18:1 yielded the addition of one ethyl group (28 Da) to the phosphocholine headgroup (Figure 4.4.5a). Upon $\mathrm{MS}^{2}$ analysis, a product ion of $\mathrm{m} / \mathrm{z} 212$ was observed with $100 \%$ relative abundance that corresponded to the ethylated PC headgroup. By preparing an equimolar solution of unmodified, methylated and ethylated PC, we clearly observed similar sensitivity increases between ethylated and methylated PC.

To address previously noted over-methylation of SM d18:1/16:0, NEU was used in place of NMU with the motivation of converging the modified analyte to a single product. Nonetheless, this gambit was unsuccessful and resulted in the addition of an ethyl group (28 Da) to the headgroup, as well as a secondary product with the addition of two ethyl groups $(56 \mathrm{Da})$. However the reaction of SM with DZM was not entirely fruitless, we directed our efforts to characterizing a mixture of ethylated and methylated SM via MS 2 .

An equimolar mixture of unmodified, methylated and ethylated SM was then produced to assess if there was a present sensitivity gain. Furthermore, it was unknown whether ethylation of SM would reduce or remove the over modification that was previously observed through methylation. As seen in Figure 4.4.5c, there was no apparent sensitivity gain from either method of alkylation. Through this, we observe the formation of an isobaric signal at $\mathrm{m} / \mathrm{z} 731.43$ due to the overlap of $[\mathrm{SM}+2 \mathrm{Me}]^{+}$and $[\mathrm{SM}+\mathrm{Et}]^{+}$. Both species can be separately detected through $\mathrm{MS}^{2}$ analysis, yielding the methylated SM headgroup at $\mathrm{m} / \mathrm{z} 198$ and the ethylated headgroup at $\mathrm{m} / \mathrm{z} 212$ (Figure 4.4.5d). Although the reaction of SM with diazoethane did not yield the desired result of decreasing the product distribution between singly and doubly alkylated SM, the results highlighted in the figure below suggests the potential of ethylation of analytes through the application of an 
iTrEnDi experimental design. The novel micro-scale gaseous reagent generator presented here shows its applicability to the use of other more harmful gaseous reagents safely.
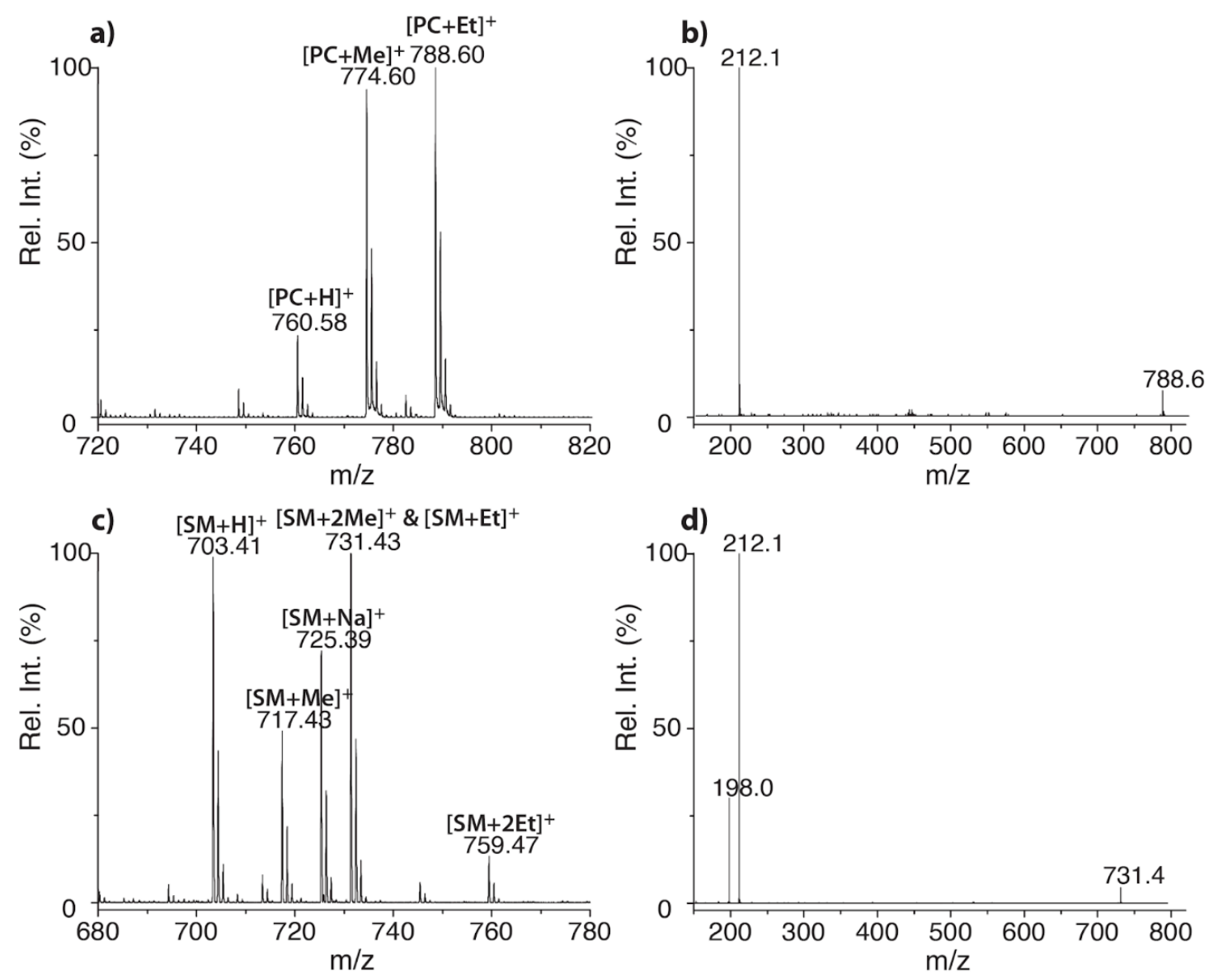

Figure 4.4.5 a) Equimolar mixture of unmodified, derivatized with DZM and derivatized with diazoethane PC 16:0/18:1, PC. b) $\mathrm{MS}^{2}$ analysis of diazoethane derivatized PC 16:0/18:1. c) Equimolar mixture of unmodified, derivatized with DZM and derivatized with diazoethane SM d18:1/16:0. d) $M^{2}$ analysis of dimethylated, $[\mathrm{SM}+2 \mathrm{Me}]^{+}$, and monoethylated, $[\mathrm{SM}+\mathrm{Et}]^{+}, \mathrm{SM}$ d18:1/16:0. 


\subsection{Conclusion}

The in situ preparation of alkylated phospholipid species results in a permanent positive charged species which in turn increases sensitivity during MS analyses. Two methods of diazoalkane preparation have been combined to give a unique approach to the micro-scale derivatization of glycerophospholipid analytes. While using iTrEnDi, the analytical chemist is protected from large quantities of toxic and explosive reagents, while also avoiding the need for specialized storage and handling equipment. Four glycerophospholipid classes were derivatized using the iTrEnDi technique and subsequently analyzed via LCMS. Each of the classes analyzed cleanly reacted with DZM to give less than 3\% residual unmodified lipid. The SM and PA classes resulted in over methylation by $11 \%$ and undermethylation by less than $1 \%$ respectively. Dimethylphosphatidic acid showed a 13.9-fold increase in sensitivity, and a dramatic improvement in chromatographic resolution. When comparing our previously reported solution based TrEnDi methodology to iTrEnDi, a modest decrease in sensitivity was observed, likely due to analyte losses during handling. However, these losses can be accepted due to the benefits of using a much safer technique. Finally, iTrEnDi methodology is more amenable to using more reactive diazoalkanes like diazoethane or other reactive gaseous reagents. Herein, we report the first use of diazoethane for the derivatization of PC and SM, resulting in the clean conversion to an ethylated lipid species bearing a fixed positive charge. Ethylation of PC standard appears to have similar increase in sensitivity as the methylated varient. Ethylated SM presents no sensitivity increase when compared to underivatized or methylated SM, however, the safer, accommodating iTrEnDi method allows for expansion of the possible chemistry for SM and other lipid classes. 


\subsection{References}

(1) Jones, J. W.; Sarkar, C.; Lipinski, M. M.; Kane, M. A. Proteomics 2019, 1800297, 1-8.

(2) Nogueras, L.; Gonzalo, H.; Jové, M.; Sol, J.; Gil-Sanchez, A.; Hervás, J. V.;

Valcheva, P.; Gonzalez-Mingot, C.; Solana, M. J.; Peralta, S.; et al. Sci. Rep. 2019, $9(1), 11313$.

(3) Van Smaalen, T. C.; Ellis, S. R.; Mascini, N. E.; Siegel, T. P.; Cillero-Pastor, B.; Hillen, L. M.; Van Heurn, L. W. E.; Peutz-Kootstra, C. J.; Heeren, R. M. A. Anal. Chem. 2019, 91 (5), 3575-3581.

(4) Bou Khalil, M.; Hou, W.; Zhou, H.; Elisma, F.; Swayne, L. A.; Blanchard, A. P.; Yao, Z.; Bennett, S. A. L.; Figeys, D. Mass Spectrom. Rev. 2010, 29 (6), 877-929.

(5) Bandu, R.; Mok, H. J.; Kim, K. P. Mass Spectrom. Rev. 2018, 37 (2), 107-138.

(6) Yang, K.; Han, X. Metabolites 2011, 1 (1), 21-40.

(7) Moncelli, M. R.; Becucci, L.; Guidelli, R. Biophys. J. 1994, 66 (6), 1969-1980.

(8) Triebl, A.; Trötzmüller, M.; Eberl, A.; Hanel, P.; Hartler, J.; Köfeler, H. C. J. Chromatogr. A 2014, 1347, 104-110.

(9) Rustam, Y. H.; Reid, G. E. Anal. Chem. 2018, 90 (1), 374-397.

(10) Fhaner, C. J.; Liu, S.; Ji, H.; Simpson, R. J.; Reid, G. E. Anal. Chem. 2012, 84 (21), 8917-8926.

(11) Wang, M.; Hayakawa, J.; Yang, K.; Han, X. Anal. Chem. 2014, 86 (4), 21462155.

(12) Wang, M.; Palavicini, J. P.; Cseresznye, A.; Han, X. Anal. Chem. 2017, 89 (16), 
8490-8495.

(13) Potter, G.; Budge, S. M.; Speers, R. A. Eur. J. Lipid Sci. Technol. 2015, 117 (7), 908-917.

(14) Sammakia, T. Diazomethane. In Encyclopedia of Reagents for Organic Synthesis; John Wiley \& Sons, Ltd: Chichester, UK, 2001.

(15) Wasslen, K. V; Tan, L. H.; Manthorpe, J. M.; Smith, J. C. Anal. Chem. 2014, 86 (7), 3291-3299.

(16) Wasslen, K. V; Canez, C. R.; Lee, H.; Manthorpe, J. M.; Smith, J. C. Anal. Chem. 2014, 86 (19), 9523-9532.

(17) Canez, C. R.; Shields, S. W. J.; Bugno, M.; Wasslen, K. V.; Weinert, H. P.; Willmore, W. G.; Manthorpe, J. M.; Smith, J. C. Anal. Chem. 2016, 88 (14), 69967004.

(18) Shields, S. W. J.; Manthorpe, J. M. J. Labelled Comp. Radiopharm. 2014, 57 (12), 674-679.

(19) Cohen, J. D.; Al-Amier, H.; Emerick, M.; Tillman, J. A.; Calio, J.; Barkawi, L. S.; Pederson, B.; Normanly, J.; Tam, Y.-Y. Anal. Biochem. 2007, 372 (2), 177-188.

(20) Barkawi, L. S.; Tam, Y. Y.; Tillman, J. A.; Normanly, J.; Cohen, J. D. Nat. Protoc. 2010, 5 (10), 1609-1618.

(21) Barkawi, L. S.; Cohen, J. D. Nat. Protoc. 2010, 5 (10), 1619-1626.

(22) Cohen, J. D. J. Chromatogr. A 1984, 303 (C), 193-196.

(23) Xia, A. J.; Kang, T. R.; He, L.; Chen, L. M.; Li, W. T.; Yang, J. L.; Liu, Q. Z. Angew. Chemie - Int. Ed. 2016, 55 (4), 1441-1444.

(24) Cajka, T.; Fiehn, O. Metabolomics 2016, 12 (2), 1-11. 
(25) Cajka, T.; Fiehn, O. TrAC - Trends Anal. Chem. 2014, 61, 192-206.

(26) Triebl, A.; Trötzmüller, M.; Eberl, A.; Hanel, P.; Hartler, J.; Köfeler, H. C. J. Chromatogr. A 2014, 1347, 104-110.

\subsection{Supporting Information}

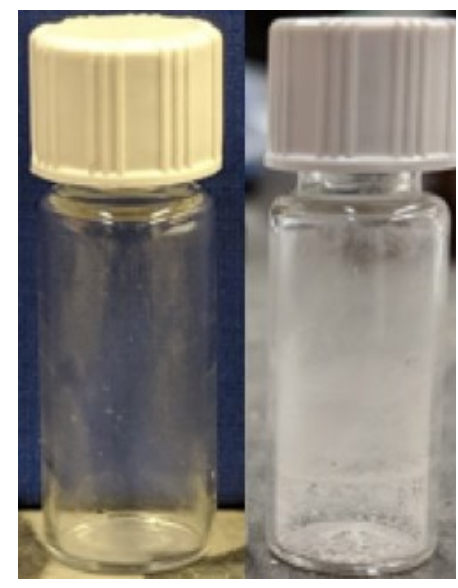
a)
b)

Figure 4.7.1 (S1) Comparison of residue left after TrEnDi (1 mL, diazomethane) modification using DZM produced from a) distilled method with Diazald ${ }^{\mathrm{TM}}$ and b) decanted method with NMU. 

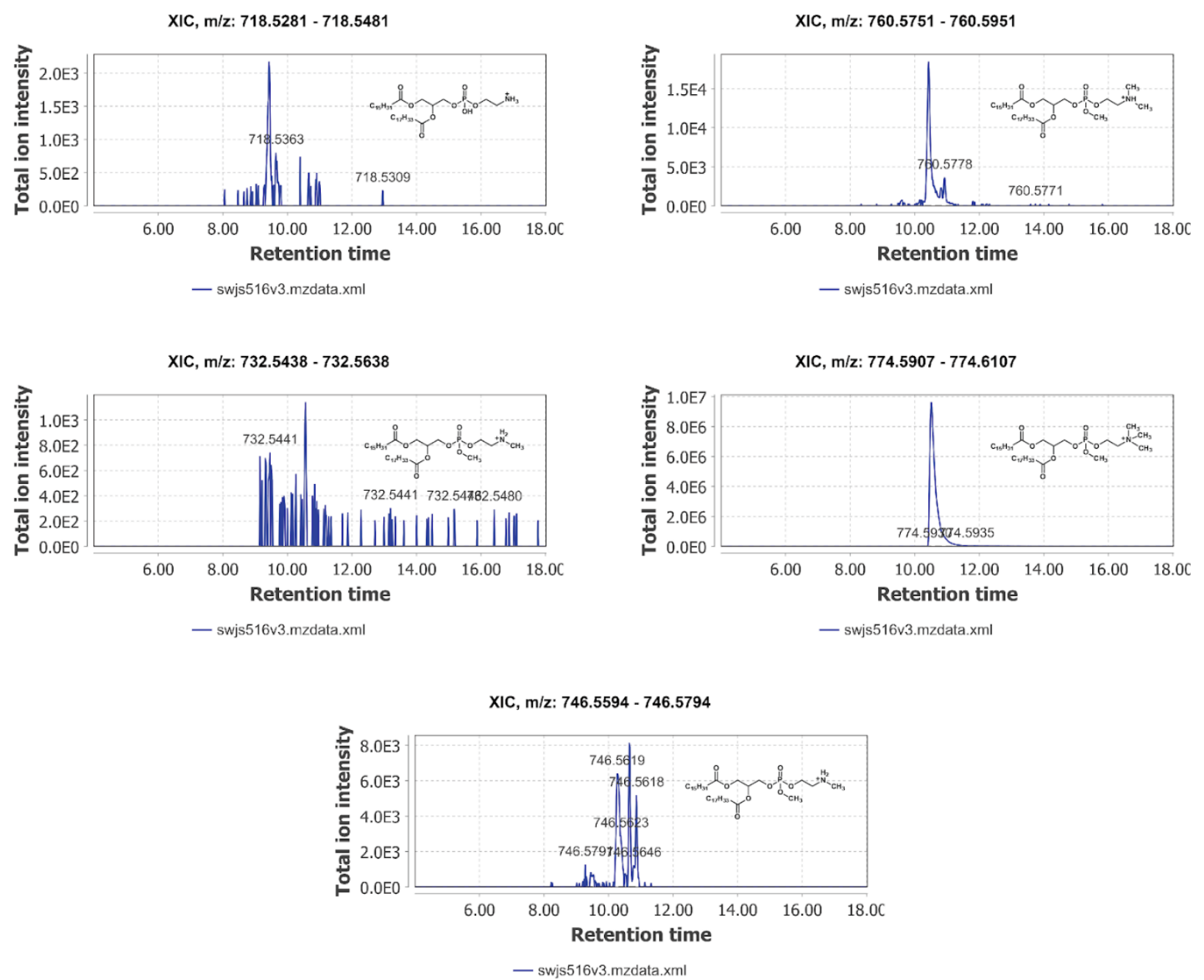

Figure 4.7.2 (S2):XICs showing undermethylated species after the iTrendi reaction of PE 16:0/18:1. 

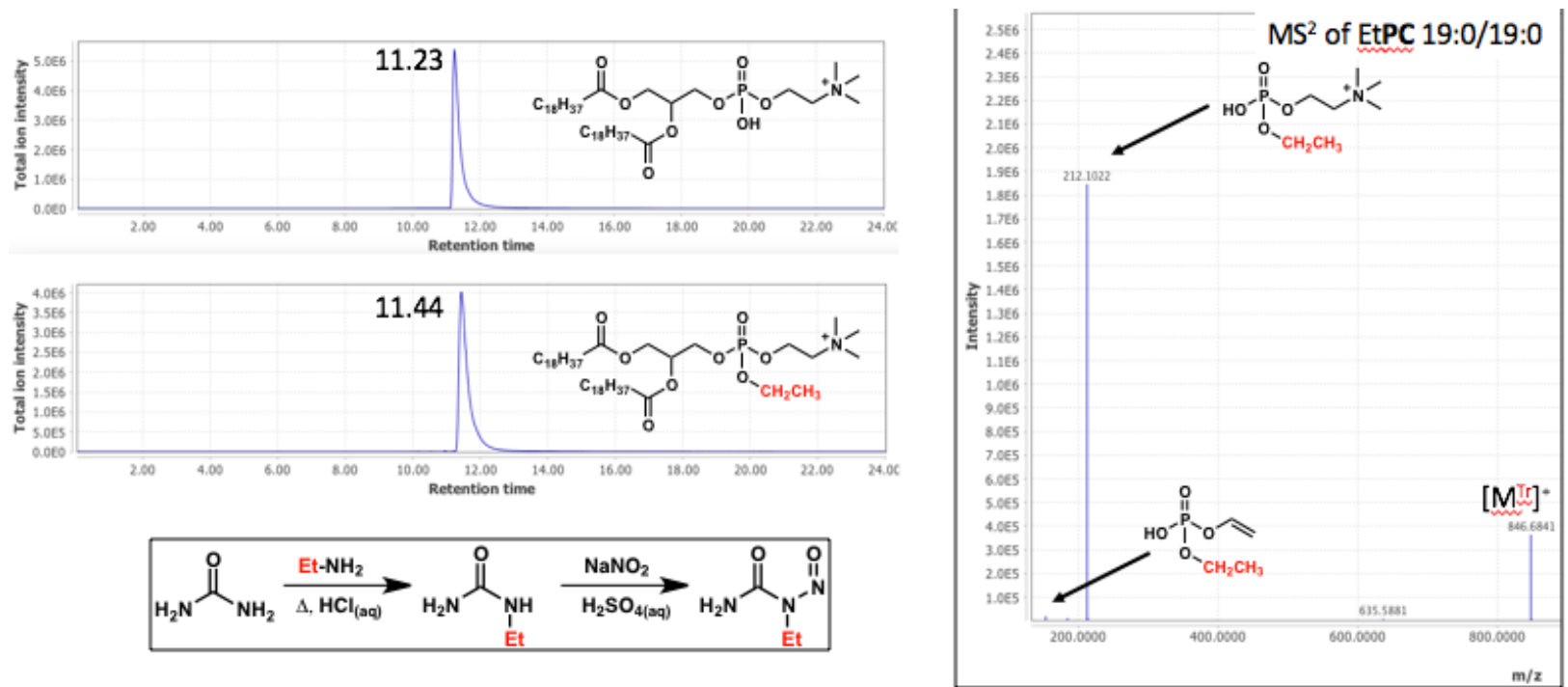

Figure 4.7.3 (S3) Chromatograms and $\mathrm{MS}^{2}$ data shown for ethylated phosphatidylcholine (PC 19:0/19:0). Unmodified PC resulted in an elution time of 11.23 min, whereas ethylated PC eluted at 11.44 min. $M^{2}$ data provided a dominant fragment at $212 \mathrm{~m} / \mathrm{z}$ represented by the modified headgroup of Et-PC.

\section{Steps S1:}

Synthesis of Diazoalkane Precursors. ${ }^{1,2,3}$<smiles>CN(N=O)C(N)=O</smiles>

Scheme 4.7.1 Preparation of $N$-methyl- $N$-nitroso urea

$N$-methyl- $N$-nitrosourea: $\mathrm{HCl}(6 \mathrm{~mL}, 37 \%)$ was slowly added to a stirred solution of methylamine hydrochloride (30.2 g 1.0 equiv., 0.45 mol, urea (89.2 g, 3.34 equiv., 1.49 mol) in $90 \mathrm{ml}$ of $\mathrm{H}_{2} \mathrm{O}$ at room temperature. The flask was fitted with a reflux condenser 
and refluxed for $3 \mathrm{~h}$. Upon cooling to room temp, $\mathrm{NaNO}_{2}(31.0 \mathrm{~g}, 1$ equiv., $0.45 \mathrm{~mol})$ was added and stirred until most of the salt was dissolved. This mixture was added slowly (2.5 h) to a mixture of ice (25 g) and $\mathrm{H}_{2} \mathrm{SO}_{4}(33.0 \mathrm{~g}, 98 \%)$, to form a yellow precipitate. During the addition, the reaction mixture was kept below $12{ }^{\circ} \mathrm{C}$. The precipitate was filtered and washed with $\sim 150 \mathrm{ml}$ of $\mathrm{H}_{2} \mathrm{O}$ or until the filtrate was neutral and dried under vacuum (100 mTorr) to yield a fine light-yellow powder $\left(22.1 \mathrm{~g}, 48 \%\right.$ overall yield). ${ }^{1} \mathrm{H}$ NMR $(300 \mathrm{MHz}$, DMSO): $\delta 8.15$ (br s, 1H), 7.82 (br s, 1H), 3.06 (s, 3H).<smiles>CCN(N=O)C(N)=O</smiles>

Scheme 4.7.2 Preparation of $N$-ethyl- $N$-nitroso urea

$N$-ethyl- $N$-nitrosourea: Was prepared in the same manner as above and yielded a light peach coloured powder (11.5 g, 23\% overall yield) ${ }^{1} \mathrm{H}$ NMR (300 MHz, DMSO): $\delta 8.09$ (br s, 1H), 7.78 (br s, 1H), 3.73 (q, $J=7.2 \mathrm{~Hz}, 2 \mathrm{H}), 0.90$ (t, $J=7.2 \mathrm{~Hz}, 3 \mathrm{H})$.

Steps S2: A step-by-step iTrEnDi setup procedure.

The experiment was performed in a fume hood, behind a polycarbonate blast shield to ensure safety at all times. Before beginning the experiment, three retort stands with clamps were prepared and evenly spaced into the back of the fume hood, one to the left, to the middle and to the right. A stir plate was placed in front of the middle retort stand and a 
high-pressure reaction vessel for a gaseous $\mathrm{N}_{2}$ source was placed to the left of the leftmost retort stand.

A $10 \mathrm{~mL}$ headspace vial was prepared, filling it with $\sim 8 \mathrm{ml}$ of diethyl ether and crimped shut to form an airtight seal. The $10 \mathrm{~mL}$ headspace vial was clamped into place on the left retort stand. About $2.5 \mathrm{~g}$ of potassium hydroxide was weighed out and placed into a $25 \mathrm{~mL}$ Erlenmeyer flask with $5 \mathrm{~mL}$ of Milli-Q water. The solution was stirred to ensure a transparent and homogeneous mixture between, then set aside to cool to room temperature to later initiate production of the diazoalkane gaseous species.

A mini freezer and an analytical balance had been placed inside of a fume hood directly next to each other, further reducing exposure to the diazoalkane forming precursors used in this experiment. In a $20 \mathrm{~mL}$ headspace vial, $2 \mathrm{~mL}$ of Milli-Q water was pipetted in. From the mini freezer, either N-Methyl-N-Nitrosourea (NMU) or N-Ethyl-N-Nitrosourea (NEU) was removed and handled cautiously in the fume hood. Roughly $100 \mathrm{mg}$ of N-alkylN-Nitrosourea was weighed out using an analytical balance and placed into the $20 \mathrm{~mL}$ headspace vial. The N-alkyl-N-Nitrosourea was resealed with parafilm and placed back into the mini freezer immediately. Approximately $1.4 \mathrm{~mL}$ of diethyl ether was pipetted into the headspace vial, with the addition of a stir bar afterwards. Once all of the contents were inside the headspace vial, a cap was crimped to the vial. The precursor solution was then clamped on the middle retort stand, being lowered to sit on the stir plate. The plates stir setting was turned up to a moderate rate to mix the precursor solution.

A $2 \mathrm{~mL}$ HPLC vial with a $250 \mu \mathrm{L}$ glass insert was used for the lipid solution. A 100 $\mu \mathrm{M}$ in $100 \mu \mathrm{L}$ standard of glycerophospholipid analyte was prepared using $\mathrm{MeOH}$ as the solvent. The lipid solution was clamped into the arm on the rightmost retort stand. 
Subsequently, in a $2 \mathrm{~mL}$ amber vial, $987 \mathrm{~mL}$ of $\mathrm{MeOH}$ was pipetted in. Tetrafluoroboric acid $\left(\mathrm{HBF}_{4}\right)$ was removed from the freezer and handled with care in the fume hood. $13 \mathrm{~mL}$ of $\mathrm{HBF}_{4}$ was added to $\mathrm{MeOH}$ and homogeneously mixed into the amber vial. Stock $\mathrm{HBF}_{4}$ was then resealed with parafilm and placed back into the freezer. The acid solution was placed aside, only adding $10 \mu \mathrm{L}$ to the lipid solution immediately before beginning the experiment. The production of these four mixtures were done in this order to ensure reproducibility of results.

Three separate lines were used to connect the entire apparatus together, allowing for a continuous flow of gaseous $\mathrm{N}_{2}$ through the system. A silica line roughly $60 \mathrm{~cm}$ long was cut to span from the ether headspace vial solution to the precursor headspace vial solution. One end of the silica line was placed into the ether solution septum, puncturing into the rightmost side of the septum, piercing about $3 \mathrm{~cm}$ into the vial. The other end of the silica line was placed into the high-pressure reaction vessel. A stream of gaseous $\mathrm{N}_{2}$ was flowed through to remove any septum that may have clogged the opposite end of the silica line. Once finished, the silica line was detached from the high-pressure reaction vessel and pierced into the leftmost side of the precursor solution septum, about $3 \mathrm{~cm}$ into the vial.

Another silica line roughly $40 \mathrm{~cm}$ long was cut and placed into the leftmost side of the ether solution vial, being pierced about $3 \mathrm{~cm}$ into the vial. The other end of the silica line was connected to the high-pressure reaction vessel. A stream of gaseous $\mathrm{N}_{2}$ was flowed through to remove any septum that may have clogged the opposite end of the silica line. $\mathrm{N}_{2}$ gas was consistently flowed at this point to create a positive pressure within the system at 5-15 psi. The end of the silica line in the ether solution vial was pushed into the ether 
solution itself, ensuring a positive flow of pressure into the lipid sample was being produced.

A syringe gauge BD PrecisionGlide ${ }^{\mathrm{TM}}$ needle (18G) was punctured into the rightmost side of the headspace solution vials septum. A Luer-Lok ${ }^{\mathrm{TM}}$ was attached to one end of PEEK tubing, with the tubing being roughly $40 \mathrm{~cm}$ in length. The Luer-Lok ${ }^{\mathrm{TM}}$ was then attached to the syringe gauge, with the other end of the PEEK tubing being placed into the lipid solution. Bubbles were present in the lipid solution from the flow of $\mathrm{N}_{2}$ gas.

Another Luer-Lok ${ }^{\mathrm{TM}}$ was attached to a piece of silica line spanning roughly $60 \mathrm{~cm}$.

The other end of the silica was placed into the center of the precursor headspace vial solution septum, piercing about $2 \mathrm{~cm}$ deep.

The acid solution was mixed well, drawing up $10 \mu \mathrm{L}$ and placing the volume into the lipid solution. With a clean syringe, $3 \mathrm{~mL}$ of base solution was drawn up. The head of the syringe was attached to the Luer-Lok ${ }^{\mathrm{TM}} /$ silica line placed in the center of the precursor headspace vial solution. Once ready, the base solution was added dropwise (1 drop/sec) into the precursor solution. The pressure was adjusted accordingly to ensure a positive flow of pressure from the gaseous $\mathrm{N}_{2}$ source. The addition of the base solution to the precursor solution produces a vibrant yellow colour in the organic layer.

Once the base solution was fully added to the precursor solution, the center silica line in the precursor solution was removed. Additionally, the leftmost silica line in the precursor solution was guided into the organic layer of the solution producing bubbles.

The experiment was monitored regularly, ensuring bubbling was constant in the ether headspace vial, the precursor headspace vial and in the lipid solution. The experiment was deemed complete once the vibrant yellow organic layer had faded, becoming a faint 
yellow to colorless layer. The precursor solution vial was left in the fume hood to vent, ensuring that any excess diazoalkane species would dissipate before handling it again.

Table 4.7.1 (S1) Complete list of materials used to assemble the iTrEnDi experimental setup.

\begin{tabular}{|c|c|l|}
\hline \multicolumn{1}{|c|}{ Item } & Quantity & \multicolumn{1}{|c|}{ Description } \\
\hline Amber Vial / Cap & $1 \mathrm{x}$ & $\begin{array}{l}\text { Fisherbrand } \\
\text { wM } \text { (Vials, Amber, Screw Thread } \\
\text { with Rubber Lined Cap, 12x35 mm, 1/2) }\end{array}$ \\
\hline Erlenmeyer Flask, 25 ml & $1 \mathrm{x}$ & Pyrex, 25 ml Erlenmeyer Flask \\
\hline Fused Silica Lines & $3 \mathrm{x}$ & $\begin{array}{l}\text { Molex, Polymicro Technologies Capillary } \\
\text { Tubing }\end{array}$ \\
\hline Fused Silica Luer-Lok & $1 \mathrm{x}$ & Luer-Lok ${ }^{\mathrm{TM}}$ Syringe Fitting \\
\hline Headspace Vial Caps & $2 \mathrm{x}$ & $\begin{array}{l}\text { Agilent Technologies (Cap, 20 mm HS AL } \\
\text { Crimp, PTFE/S 100 PK) }\end{array}$ \\
\hline HPLC Cap & $1 \mathrm{x}$ & $\begin{array}{l}\text { Agilent Technologies (Cap, 9 mm Blue Screw, } \\
\text { PTFE/RS, 500PK) }\end{array}$ \\
\hline HPLC Insert & $1 \mathrm{x}$ & $\begin{array}{l}\text { Canadian Life Science (250 } \mu \mathrm{L} \text { Glass Conical } \\
\text { Insert Bottom Spring, 6x29mm) }\end{array}$ \\
\hline HPLC Vial & $1 \mathrm{x}$ & $\begin{array}{l}\text { Agilent Technologies (Vial, Screw, 2 ml, } \\
100 P K)\end{array}$ \\
\hline Large Headspace Vial & $1 \mathrm{x}$ & $\begin{array}{l}\text { Agilent Technologies (Vial, Headspace, Crimp, } \\
\text { FB, 20 ml, 100PK) }\end{array}$ \\
\hline
\end{tabular}




\begin{tabular}{|c|c|c|}
\hline $\mathrm{N}_{2}$ Gas Tank & $1 \mathrm{x}$ & $\begin{array}{l}\text { Praxair Canada Inc., Compressed Nitrogen, } \\
\text { GP-520076A }\end{array}$ \\
\hline PEEK Capillary Tubing & $1 \mathrm{x}$ & $\begin{array}{l}\text { ThermoFisher Scientific Capillary Tubing, } \\
0.025 \mathrm{~cm} \text { ID }\end{array}$ \\
\hline PEEK Fitting & $1 \mathrm{x}$ & Peek 1/16" Standard Tubbing Fittings \\
\hline $\begin{array}{l}\text { Polycarbonate Blast } \\
\text { Shield }\end{array}$ & $1 \mathrm{x}$ & $\begin{array}{l}\text { Bel-Art SP Scienceware, Large Weighted } \\
\text { Safety Shield }\end{array}$ \\
\hline Pressure Bomb & $1 \mathrm{x}$ & High Pressure Reaction Vessel ${ }^{4}$ \\
\hline Retort Stands & $3 x$ & VWR Retort Stands \\
\hline Small Headspace Vial & $1 \mathrm{x}$ & $\begin{array}{l}\text { Agilent Technologies (Vial, Headspace, Crimp, } \\
\text { FB, } 10 \mathrm{ml}, 100 \mathrm{PK} \text { ) }\end{array}$ \\
\hline Stir-bar & $1 \mathrm{x}$ & $\begin{array}{l}\text { Fisherbrand }^{\mathrm{TM}} \text { Octagon Spinbar } \\
\text { Stirring Bars, } 2.54 \mathrm{~cm} \text { ) }\end{array}$ \\
\hline Stir Plate & $1 \mathrm{x}$ & VWR, Model 320 \\
\hline Syringe & $1 \mathrm{x}$ & $\begin{array}{l}\text { BD Disposable } 3 \mathrm{ml} \text { Syringe with Luer-Lok }{ }^{\mathrm{TM}} \\
\text { Tips }\end{array}$ \\
\hline Syringe Gauge & $1 \mathrm{x}$ & $\begin{array}{l}\text { BD PrecisionGlide }{ }^{\mathrm{TM}} \text { Needle, } 18 \mathrm{G} \times 1 \frac{1}{2} \\
(1.2 \mathrm{~mm} \times 40 \mathrm{~mm})\end{array}$ \\
\hline
\end{tabular}

References:

1. Foley, D. A.; O’Leary, P.; Buckley, N. R.; Lawrence, S. E.; Maguire, A. R.

Tetrahedron. 2013, 69, 1778-1794.

2. Org. Synth. 1935, 15, 43. 
3. Xia, A.; Kang, T.; He, L.; Chen, L.; Li, W.; Yang, J.; Liu, Q. Angewandte Chemie International Ed. 2016, 55, 4, 1441-1444.

4. Wasslen, K. V.; Tan, L. H.; Manthorpe, J. M.; Smith, J. C. Anal. Chem. 2014, 86, 3291-3299. 


\title{
Chapter 5: Determination of Glyphosate and Aminomethylphosphonic Acid Using A Novel Chemical Derivatization Technique and Analysis via LCMS
}

\author{
Samuel W. J. Shields ${ }^{\mathrm{a}, \mathrm{c}}$, Christian A. Rosales ${ }^{\mathrm{a}, \mathrm{c}}$, Chelsey L. J. Aulenback ${ }^{\mathrm{a}, \mathrm{b}, \mathrm{c}}$, Peter J. \\ Pallister $^{\mathrm{a}, \mathrm{c}}$, Karl V. Wasslen ${ }^{\mathrm{a}, \mathrm{c}}$ Jeffrey M. Manthorpe ${ }^{\mathrm{a}, \mathrm{b}, \mathrm{c} *}$, Jeffrey C. Smith ${ }^{\mathrm{a}, \mathrm{b}, \mathrm{c} *}$ \\ a) Department of Chemistry Carleton University, Ottawa, Ontario K1S 5B6, \\ Canada \\ b) Institute of Biochemistry, Carleton University, Ottawa, Ontario K1S 5B6, \\ Canada \\ c) Carleton Mass Spectrometry Centre, Carleton University, Ottawa, Ontario \\ K1S 5B6, Canada
}

*to whom correspondence should be addressed - jeff.smith@carleton.ca, jeffrey.manthorpe@carleton.ca

Keywords: herbicides, glyphosate, aminomethylphosphonic acid, diazomethane, mass spectrometry

\section{$5.1 \quad$ Abstract}

Glyphosate (GLY) containing herbicides are the most commonly used weed and vegetation control products in the world, which are used in billions of kilograms annually. There have been growing concerns about accumulation in the environment and increased human exposure in the literature. The emergence of chemical derivatization strategies coupled with liquid chromatography mass spectrometry (LCMS) techniques have emerged 
to address the challenges of quantifying low levels of GLY and aminomethylphosphonic acid (AMPA) in complex biological samples. In this article, we aim to show the utility of in situ Trimethylation Enhancement Using Diazomethane (iTrEnDi) to derivatize GLY and AMPA by fixing a positive charge to both species prior to analysis via LCMS with multiple reaction monitoring (MRM). Methylated GLY and AMPA standards were produced in greater than $99 \%$ conversion to a single product that were readily identified by tandem HRMS. After the optimization of MRM conditions, good reproducibility of the iTrEnDi reaction was observed and the fold increase due to methylation was determined to be 12.4and 339.7-fold for GLY and AMPA, respectively. Furthermore, LOD and LOQ for each derivatized compound were observed to be in the low $\mathrm{ng} / \mathrm{L}$ range and could be detected on the exterior of soybeans after a simple aqueous extract. Although the analysis of these small molecular weight herbicides is challenging using conventional LCMS techniques, our derivatization method shows great promise in overcoming such challenges as well as having the potential to further comprehend their environmental and biological fates.

\subsection{Introduction}

$N$-(phosphonomethyl)glycine or glyphosate (GLY) is a water broad-spectrum herbicide that interacts strongly in the shikimate pathway, reducing a plants ability to produce aromatic amino acids causing the buildup of toxic intermediates in the pathway, overall causing death. ${ }^{1}$ It has been used as a herbicide for the promotion of efficient crop production around the world since the early 1980s and continues today as one of the most commonly used products on the market for vegetation control. The main formulation "Roundup" is sold as a isopropylamine salt with polyoxyethylene amine surfactants to 
improve solubility and efficiency. ${ }^{2}$ Approximately, 1.6 billion kg of glyphosate has been used in the US since 1974, with two thirds being applied in the past ten years alone. ${ }^{3}$ Global use of GLY products has become ubiquitous in the modern era of agriculture, but its exponentially increasing usage over time is concerning as the environmental and toxicological effects become more apparent.

Aminomethylphosphonic acid (AMPA) is the main degradation product of GLY and has been conjectured to form through metabolism in soil microorganisms (Scheme 5.2.1). ${ }^{4}$ Both GLY and AMPA are highly water soluble polar molecules that are known to accumulate in water surrounding agricultural sites and beyond. ${ }^{5-7}$ Although GLY and AMPA have been treated as a "toxicologically harmless", recent studies suggest exposure can affect cell cycle regulation in mammals ${ }^{9}$, nitrogen fixing pathways in soil microbes ${ }^{1}$, and the development of organs in aquatic life. ${ }^{10}$ Furthermore, GLY and AMPA have been found in bioactive concentrations in human urine ${ }^{11}$ and breast milk ${ }^{12}$ likely due to bioaccumulation in drinking water and vegetables.

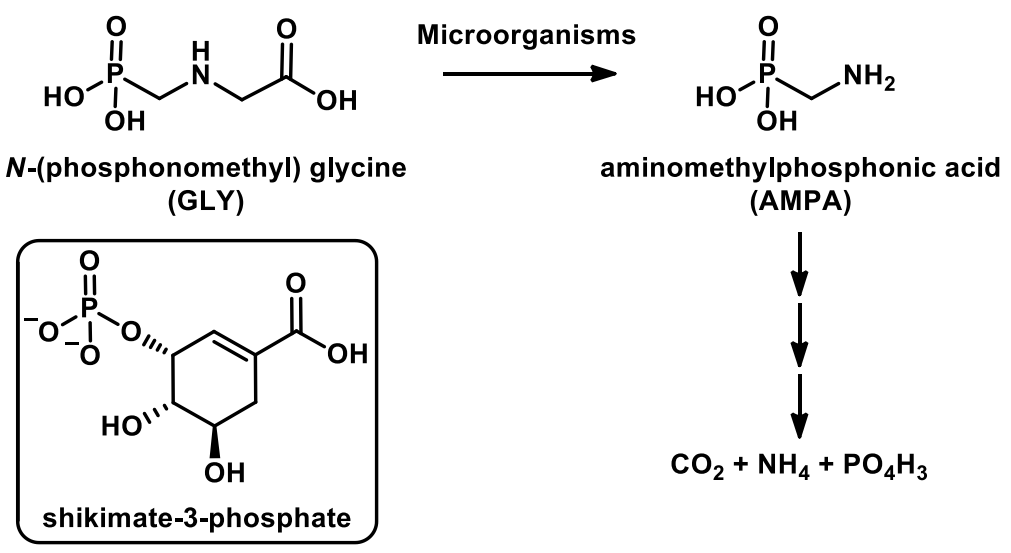

Scheme 5.2.1 Proposed Degradation of Glyphosate in Soil by Microorganisms 
There are several challenges with the determination of GLY in biological matrices due to the small size, high polarity, low volatility, low solubility in organic solvents, and structural similarity to naturally occurring amino acids. ${ }^{2,5}$ Liquid chromatography mass spectrometry (LCMS) is the emerging technology adopted by researchers to detect and analyze GLY, however due to the chemical and physical characteristics previously mentioned, LCMS determination requires techniques such as ion or normal phase LC, and detection in negative ion mode MS. One major difficulty for studies using quantitative LCMS analyses of GLY and AMPA is the narrow scope the current methods offer in terms of analyzing multiple other analytes in the same assay. The limited techniques leads to GLY being one of the most widely used agrochemical in the world as well as being one of the most rarely determined, this is commonly referred to as the "glyphosate paradox".,25

Chemical derivatization is a common solution to improve chemical characteristics of GLY and AMPA for their analysis via LCMS. The preparation of carbamates and urea derivatives of glyphosate is a routine method for increasing sensitivity in LCMS analysis. This year, quantitative analyses of GLY have been performed using 9fluorenylmethoxycarbonyl chloride ${ }^{13}$ (FMOC-Cl) and 6-aminoquinolyl- $N$ hydroxysuccinimidyl carbamate ${ }^{7}$ (AQC) to achieve sub microgram per liter limits of detection (Scheme 5.2.2). Some major disadvantages of current derivatization strategies are the large reagent loading per sample $(0.2-3.0 \mathrm{mg})$, the need for post modification clean-up, and the production of unwanted by-products. In general, derivatization techniques for absolute quantitation of GLY and AMPA requires that any reaction must be rapid, high yielding, and afford minimal side products to truly investagate their environmental and biological fates. 
An emerging derivatization technique, Trimethylation Enhancement Using Diazomethane (TrEnDi), has become a novel method to enhance sensitivity of peptides ${ }^{14}$ and lipids ${ }^{15,16}$ through the methylation of amino, carboxyl, and phosphate groups to generate a fixed, permanent positive charge. Diazomethane (DZM) is a highly reactive reagent that reacts cleanly with functional groups that have $\mathrm{pK}_{\mathrm{a}}$ less than 11 to produce only nitrogen gas as a by-product. An in situ variant of TrEnDi (iTrEnDi) has recently been developed to produce small amounts of DZM for the methylation of polar aqueous soluble analytes with minimal handling and storage of the hazardous reagent (Chapter 4). In this paper we aim to increase the sensitivity of GLY and AMPA in multi-substrate compatible LCMS analyses through derivatization using the iTrEnDi methodology.

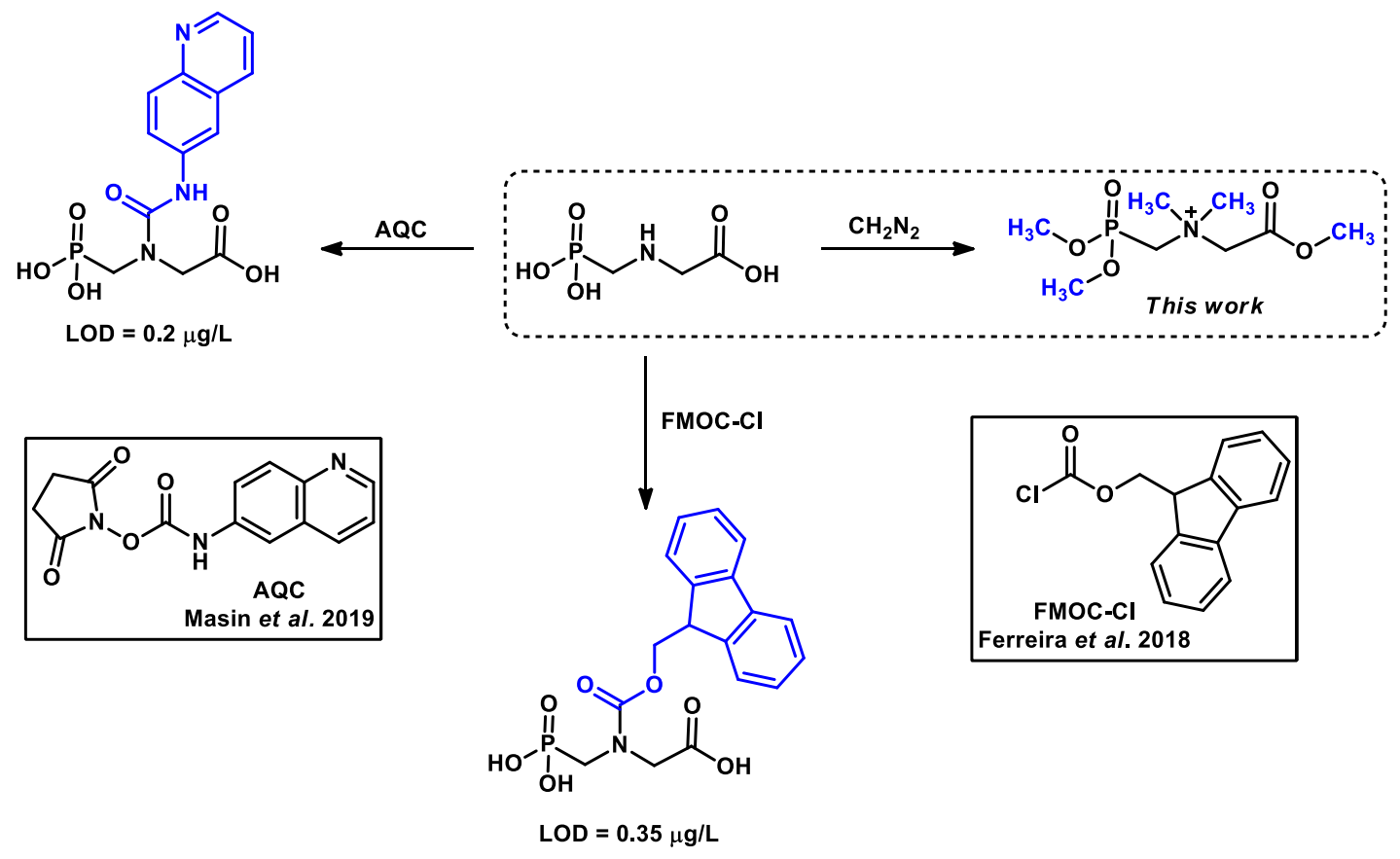

Scheme 5.2.2 Recent derivatization strategies for LCMS analysis of glyphosate and AMPA 


\subsection{Experimental}

Chemicals and Materials. $N$-(phosphonomethyl)glycine, aminomethylphosphonic acid, diethylene glycol methyl ether, caffeine and $\mathrm{HBF}_{4} \bullet \mathrm{Et}_{2} \mathrm{O}$ were purchased from SigmaAldrich (St. Louis, MO, USA). LCMS grade water with $0.1 \%$ formic acid, acetonitrile with $0.1 \%$ formic acid and methanol were purchased from Fisher scientific (Hampton, $\mathrm{NH}$, USA). $\mathrm{KOH}$ and $\mathrm{Et}_{2} \mathrm{O}$ were purchased from Caledon Laboratories Ltd. (Georgetown, ON, Canada). Roundup ${ }^{\circledR}$ was purchased from a local hardware store and soybean plants treated with roundup were obtained from Myron Smith at Carleton University.

iTrEnDi derivatization of samples. CAUTION: All reactions involving preparation and derivatization with diazoalkanes should be performed in a chemical fume hood with efficient ventilation and behind a polycarbonate blast shield due to their toxic and explosive nature. Diazomethane precursor $N$-methyl- $N$-nitroso- $p$-toluenesulfonamide and N-Methyl-N'-nitrosourea were prepared according to literature procedures. ${ }^{17,18}$ For a complete visual representation of the experimental setup, refer to Figure S1. A solution of aqueous $\mathrm{KOH}(1 \mathrm{ml}, 6.1 \mathrm{M})$ was added into a sealed headspace vial dropwise (1 drop/s) into a solution of diethylene glycol methyl ether : diethylether $(1: 1 \mathrm{v} / \mathrm{v})$ and $N$-methyl- $N$ nitroso- $p$-toluenesulfonamide $(0.4 \mathrm{~g}, 1.87 \mathrm{mmol})$ at room temperature. The diazoalkane $/ \mathrm{N}_{2}$ output line of the reactor was inserted into $150 \mu \mathrm{L}$ methanol solution containing $\mathrm{HBF}_{4} \bullet \mathrm{Et}_{2} \mathrm{O}$ $(8 \mu \mathrm{L}, 0.8 \mu \mathrm{mol})$, glyphosate $(5 \mathrm{nmol})$ and AMPA $(5 \mathrm{nmol})$. Upon the addition of aqueous $\mathrm{KOH}$, constant effervescence was observed. After the addition of base was complete, a vivid yellow solution was obtained and $\mathrm{N}_{2}$ (in) gas line was adjusted to be submerged by at least $1 \mathrm{~cm}$ under the surface. The reaction proceeded at room temperature for $1 \mathrm{~h}$ before a second aliquot of $\mathrm{HBF}_{4} \cdot \mathrm{Et}_{2} \mathrm{O}(0.8 \mu \mathrm{mol})$ was added. Once minimal yellow color in the 
headspace vial was observed ( $\sim 1 \mathrm{~h}$ after the second addition of acid) the apparatus could be disassembled safely. Residual solvent was evaporated via a stream of $\mathrm{N}_{2}(\mathrm{~g})$ and stored at $-20{ }^{\circ} \mathrm{C}$. Alkylated pesticide samples were resuspended in $\mathrm{MeOH}$ and analyzed immediately by LCMS.

Reverse Phase High Pressure Liquid chromatography. Twenty $\mu \mathrm{L}$ of pesticide sample $25 \mu \mathrm{M}$ unmodified or $5 \mu \mathrm{M}$ methylated standards) was injected onto an Agilent Eclipse plus $\mathrm{C} 183.5 \mu \mathrm{m}, 4.6 \times 100 \mathrm{~mm}$ column at room temperature using the autosampler of a Thermo Scientific Dionex Ultimate 3000 HPLC. A binary gradient program consisting of water plus $0.1 \%$ formic acid (mobile phase A) and acetonitrile plus $0.1 \%$ formic acid (mobile phase B). Flow rate was maintained at $0.6 \mathrm{ml} / \mathrm{min}$ and the separation gradient was: 0.0-1.5 min, $0.5 \%$ (B); 7.0-9.8 $\min , 100 \%$ (B); $10.0-12.0 \mathrm{~min}, 0.5 \%$ (B).

ESI-MS analysis. The eluent was introduced into a Sciex Qtrap 4000 fitted with a Turbo $\mathrm{V}$ ionspray source at $375{ }^{\circ} \mathrm{C}$ in positive mode with an ionization voltage of $+5000 \mathrm{~V}$, curtain gas of 20, nebulizing gas of 30 , and drying gas of 10 . All samples were analyzed using MRM scans at $50 \mathrm{~ms}$ each with transitions and Q3 parameters found in Table 5.3.1. 
Table 5.3.1 MRM parameters used for LCMS analysis

\begin{tabular}{|c|c|c|c|c|c|}
\hline Transition & $\begin{array}{l}\text { Precursor } \\
\text { Ion }(\mathrm{m} / \mathrm{z})\end{array}$ & $\begin{array}{c}\text { Product } \\
\text { Ion }(\mathrm{m} / \mathrm{z})\end{array}$ & $\mathrm{CE}(\mathrm{V})$ & $\mathrm{DP}(\mathrm{V})$ & $\begin{array}{l}\text { CXP } \\
\text { (V) }\end{array}$ \\
\hline $\begin{array}{l}\text { Glyphosate } \\
\text { quantifier }\end{array}$ & 170.0 & 88.0 & 13 & 35 & 7 \\
\hline $\begin{array}{l}\text { Glyphosate } \\
\text { Qualifier }\end{array}$ & 170.0 & 42.0 & 28 & 35 & 7 \\
\hline $\begin{array}{c}\text { AMPA } \\
\text { Quantifier }\end{array}$ & 112.0 & 30.0 & 17 & 35 & 4 \\
\hline $\begin{array}{l}\text { AMPA } \\
\text { Qualifier }\end{array}$ & 112.0 & 83.0 & 20 & 35 & 3 \\
\hline $\begin{array}{l}\text { Mes-Glyphosate } \\
\text { quantifier }\end{array}$ & 240.1 & 58.0 & 50 & 57 & 9 \\
\hline $\begin{array}{l}\text { Mes-Glyphosate } \\
\text { Qualifier }\end{array}$ & 240.1 & 116.0 & 28 & 57 & 9 \\
\hline $\begin{array}{l}\text { Mes-AMPA }_{5} \text { quantifier }\end{array}$ & 182.0 & 58.0 & 50 & 50 & 9 \\
\hline $\begin{array}{l}\text { Me5-AMPA } \\
\text { Qualifier }\end{array}$ & 182.0 & 93.0 & 37 & 47 & 6 \\
\hline $\begin{array}{l}\text { Caffeine } \\
\text { quantifier }\end{array}$ & 195.1 & 138.1 & 28 & 75 & 10 \\
\hline $\begin{array}{l}\text { Caffeine } \\
\text { Qualifier }\end{array}$ & 195.1 & 110.1 & 28 & 75 & 10 \\
\hline
\end{tabular}

nESI-MS $^{2}$ analysis. Six $\mu \mathrm{L}$ of resuspended modified glyphosate and AMPA was inserted into a Proxeon nano-electrospray emitter (Thermo Scientific) and analysed in positive mode (m/z 10-300) by direct spray using a QStarXL (Sciex) equipped with a nano-ESI source. Analysis parameters were as follows; Nano-ESI voltage of $1400 \mathrm{~V}$, declustering and focusing potentials of $30 \mathrm{~V}$ and $120 \mathrm{~V}$, respectively, and $\mathrm{MS}^{2}$ collision energy of 35 $\mathrm{eV}$ for methylated AMPA and 50 for methylated glyphosate. 
Extraction of glyphosate from soybean pods. Two grams (fresh weight) of soybean plant material was extracted with $10 \mathrm{~mL}$ of $\mathrm{H}_{2} \mathrm{O}$ for $30 \mathrm{~min}$ at room temperature. Five $\mathrm{mL}$ of extract was centrifuged at $1000 \mathrm{xg}$ for $10 \mathrm{~min}$ before analysis via MS.

\subsection{Results and Discussion}

With the more classical LCMS conditions such as, using acetonitrile/water with $0.1 \%$ formic acid as solvents, positive ion mode, and the use of C18 stationary phase detection and quantitation of glyphosate and AMPA is challenging. Figure 5.4.1A, shows the sodium adduct formation of both compounds in MS+, causing the signal in MS to split significantly between protonated and sodiated adducts. The retention of these compounds on $\mathrm{C} 18$ reverse phase columns is very low; Figure 5.4.1B shows both glyphosate and AMPA analyzed via a tandem MS approach in positive ion mode, having virtually no retention when accounting for solvent delay. The ability to remove metal cations on column using a sustained flow of water is not possible with GLY and AMPA due to their poor retention. When these analytes are contained within a complex matrix such as plant or animal extracts many low $\mathrm{m} / \mathrm{z}$ ions and metal cations will obfuscate their analysis. Glyphosate especially, is known to chelate strongly with metal cations, which are present in aqueous extracts and cause low signal intensities due to adduct formation with LCMS analysis in positive ion mode. ${ }^{19}$ An alternative procedure is to use MS in negative ion mode, which takes advantage of the gas phase acidity of phosphonic/carboxylic acids, to become readily deprotonated. ${ }^{20}$ Figure $5.4 .1 \mathrm{C}$ is shows the MRM analysis of GLY and AMPA in negative ion mode, using previously reported transitions, ${ }^{12}$ having a larger total peak area. This increase in peak area can be attributed to the increase ionization efficiency and 
consolidation of any metal cation adducts. The analysis of underivatized GLY and AMPA in negative ion mode generally requires hydrophobic interaction chromatography ${ }^{21}$ or anion exchange chromatography ${ }^{22}$ that are incompatible with the analysis of other herbicide analysis, require long equilibration steps and lengthy solvent optimization.
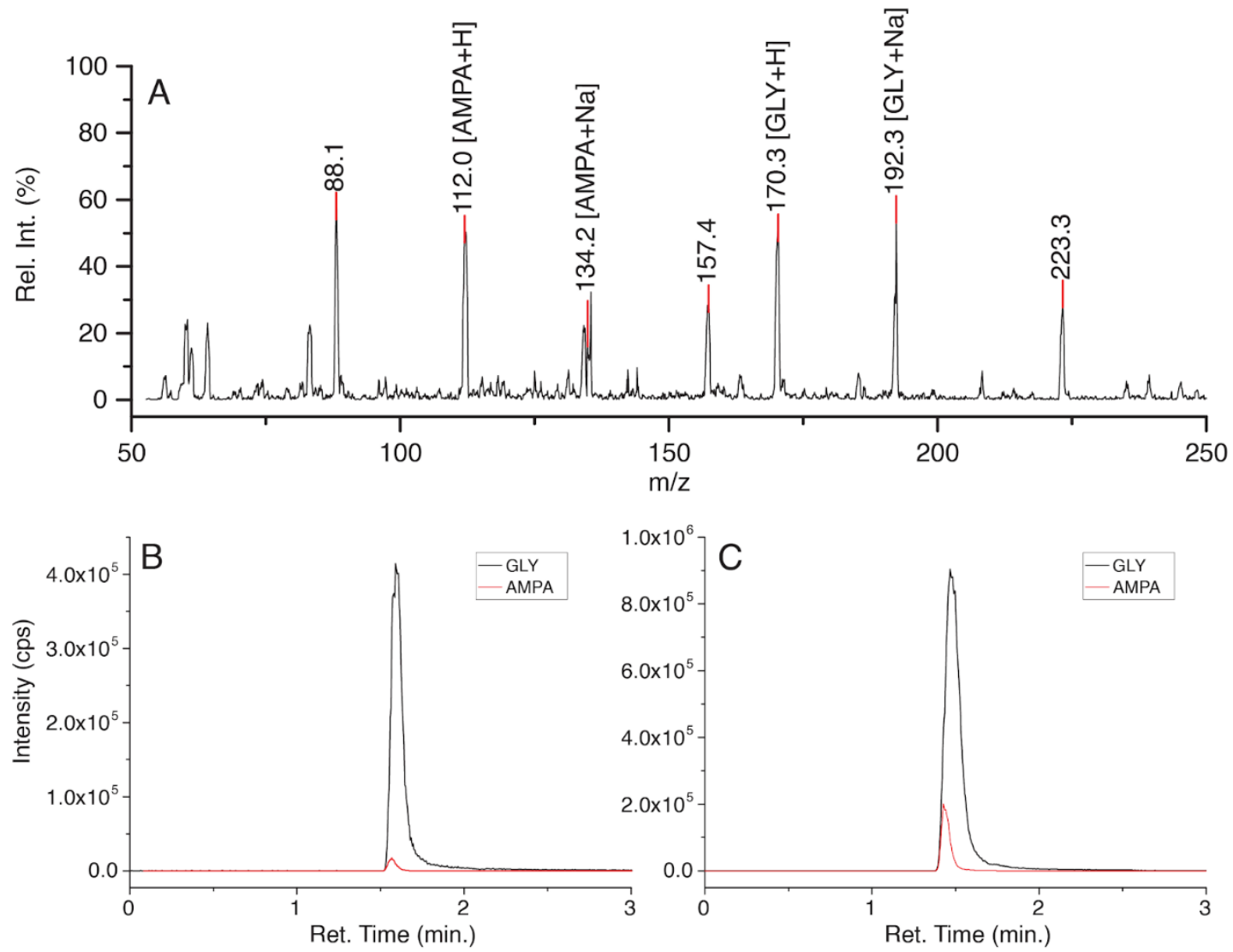

Figure 5.4.1 Signal splitting of glyphosate and AMPA between protonated and sodiated adducts in +MS (A). LCMS analysis of glyphosate and AMPA in +MS (B) and -MS (C) using multiple reaction monitoring.

Many derivatization protocols for the analysis of glyphosate use the carboxylic acid or amine functionality of GLY as a handle for installing non-polar groups or chromophores to improve LCMS and UV detection. ${ }^{13,23}$ But alkylation using iTrEnDi methodology 
employs the rapid derivatization of amines, phosphonic, phosphoric, carboxylic acid components with gaseous diazomethane. This technique has been shown to effectively alkylate phosphatidylethanolamine at its phosphate and amine groups to render it a fixed positive charged species (Chapter 4). Initial reaction conditions of GLY and DZM resulted in a distribution of methylation states produced are highlighted in Figure 5.4.2B. The spectrum produced by direct ESI analysis shows the presence of three methylation products, identified by $\mathrm{m} / \mathrm{z}$ as Me3-GLY (m/z 212), Me4-GLY (m/z 226) and Me5-GLY (m/z 240) with Me4-GLY being the most abundant. Since our goal was to produce one methylated product to provide the largest increase in signal, we had to revise the original reaction conditions. Generally, the protonation of diazomethane has been measured to be slower than the displacement of $\mathrm{N}_{2}$ by a nucleophile,${ }^{24}$ it would follow that the $\mathrm{pKa}$ of the acid reacting with DZM would play a role in the overall rate of reaction. From this we can hypothesize the order of methylation of GLY with DZM to be the phosphoric acids, carboxylic acid then ammonium species thus Me4-GLY, would have one methylation site at $N$ vacant.

Ultimately, we were most interested in the production of Mes-GLY since this structure contains a fixed positive charge and would yield the greatest ionization efficency during LCMS analysis. The high resolution $\mathrm{MS}^{2}$ spectrum was obtained to fully characterize Me5-GLY to be the desired product (Figure 5.4.2D). Scheme 5.4.1 shows the reaction products identified in the initial TrEnDi derivatization of GLY and the proposed fragmentation pathways for Mes-GLY. The main two fragmentation channels are proposed to be McLafferty style rearrangements involving either the carbonyl side to yield $\mathrm{m} / \mathrm{z} 166$ or the phosphate ester to give $\mathrm{m} / \mathrm{z} 116$. Although the fragmentation pathway to $\mathrm{m} / \mathrm{z} 58$ is 
unclear, its molecular formula of $\mathrm{C} 3 \mathrm{H} 8 \mathrm{~N}^{+}$determined by HRMS, can be produced from multiple intermediates thus making it the most abundant. Tandem high-resolution MS (HRMS) analysis confirms the identity of $\mathrm{m} / \mathrm{z} 240$ as Mes-GLY and produces some molecular fragment transitions that were used for further MRM analysis.

Tandem MS analysis of the Me4-GLY (Figure 5.4.2C) revealed a major fragmentation of $\mathrm{m} / \mathrm{z} 194$ which corresponds to the loss of $\mathrm{MeOH}$ and the production of the corresponding acylium ion. By using the transition of $\mathrm{m} / \mathrm{z} 226 \rightarrow \mathrm{m} / \mathrm{z} 194$, the resulting SRM chromatogram in Figure 5.4.2A was produced. Interestingly, there is a small feaature that elutes just before the bulk of Me4-GLY which could be an indication of a small amount of an alternate methylation site isomer produced during the reaction. There are practically three major isomers, where either the phosphate position, carboxylic acid or amine positions are unmethylated. This hypothesis is supported by the $\mathrm{MS}^{2}$ fragments of $\mathrm{m} / \mathrm{z} 166$ and $\mathrm{m} / \mathrm{z} 152$ in Figure 5.4.2C, which from the fragmentation analysis of Mes-GLY are the proposed McLafferty products resulting in the loss of carboxyl portion of the molecule, and a similar fragment that is $14 \mathrm{Da}$ less. The $\mathrm{m} / \mathrm{z}$ of 152 is produced from the isomer with tertiary ammonium species, whereas $\mathrm{m} / \mathrm{z} 166$ would be produced from an isomer bearing a quaternary ammonium species. Despite, the production of multiple methylated products, we were confident that the full conversion of GLY to Mes-GLY could be attained through the optimization of reaction conditions. 

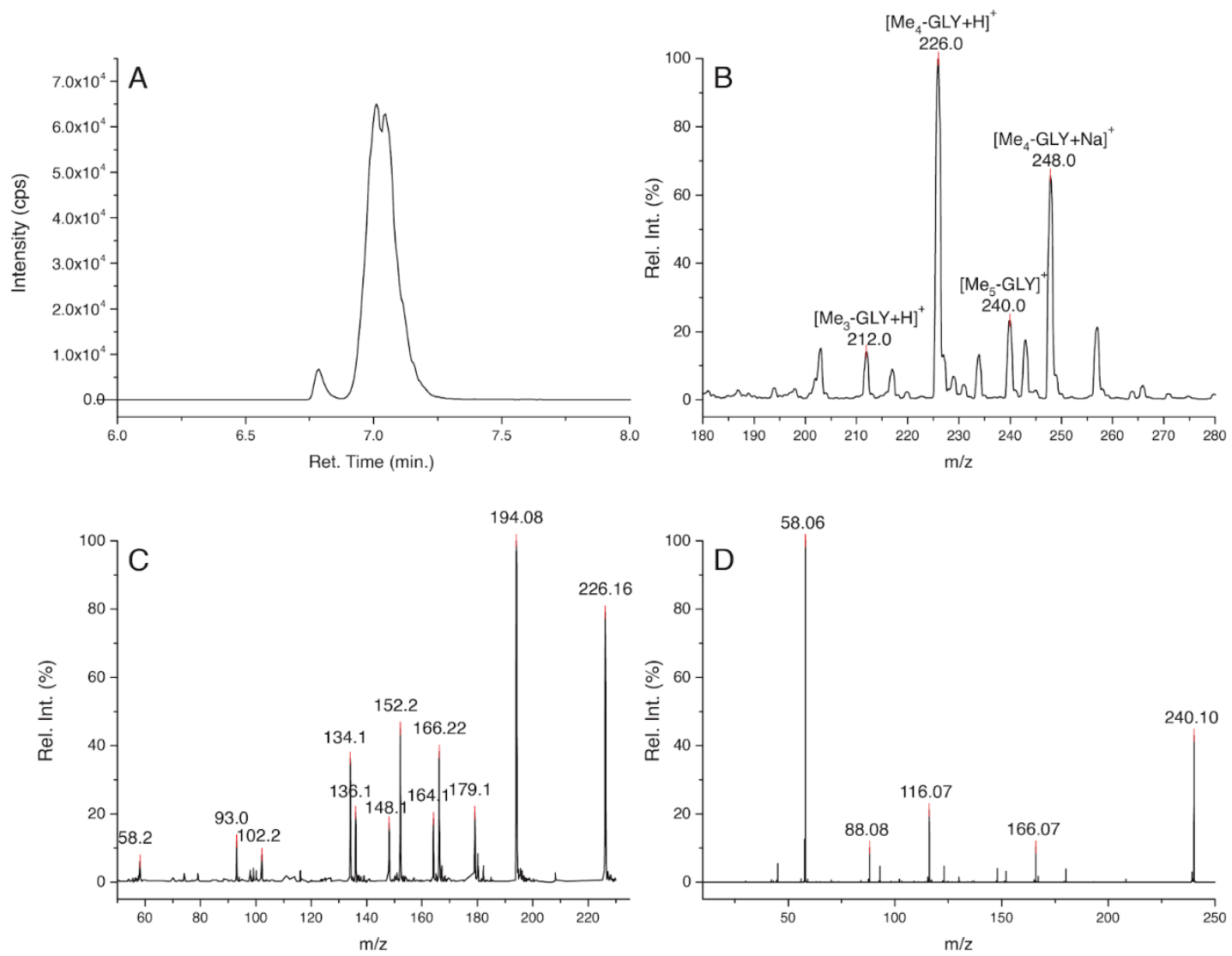

Figure 5.4.2 LCMS analysis of $\mathrm{Me}_{4}$-glyphosate using multiple reaction monitoring (A). Direct + MS of the reaction mixture produced from the iTrEnDi derivatized glyphosate (B). The MS' of Me4- (C) and Me5- (D) GLY using LCMS and direct nESI-MS, respectively. 


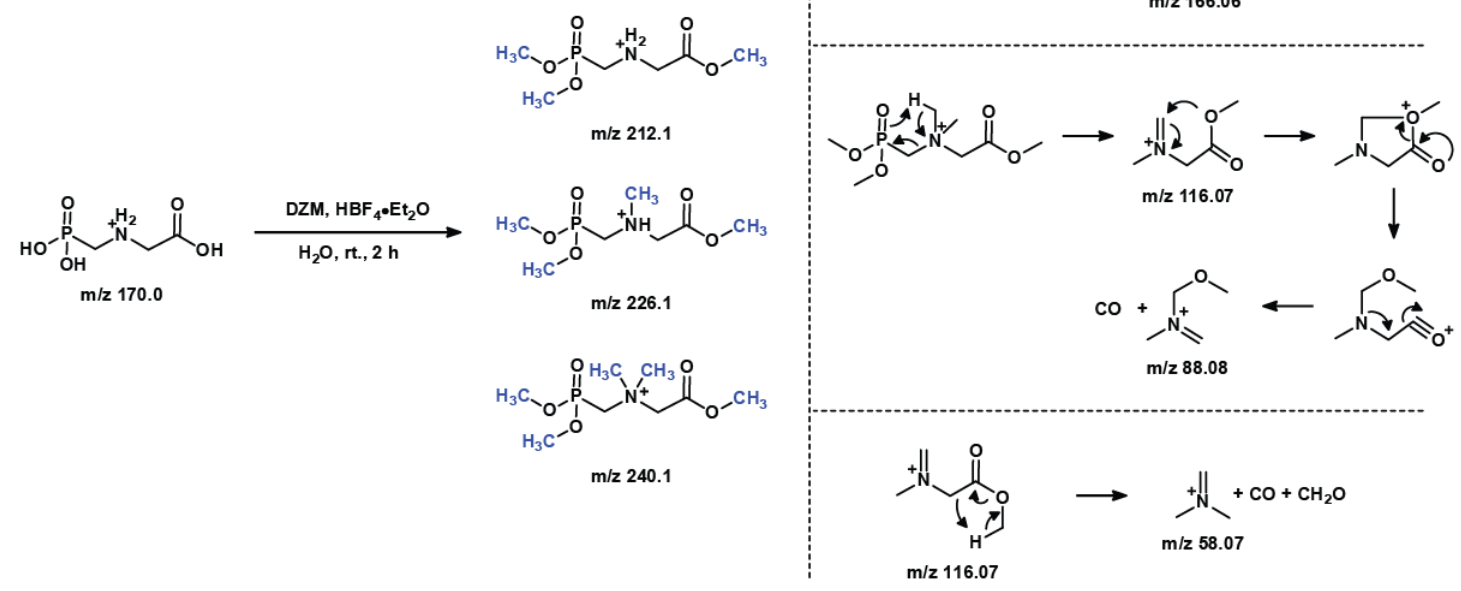

Scheme 5.4.1 Reaction products produced by the reaction of DMZ and glyphosate (left). Proposed fragmentation mechanisms for $\mathrm{MS}^{2}$ fragments observed for $\mathrm{Me}_{5}$ glyphosate (right).

Since initial iTrEnDi reaction with GLY conditions used $N$-methyl- $N$-nitrosourea as a DZM precursor and $\mathrm{H}_{2} \mathrm{O}$ as a solvent for GLY we opted to start with modifying these parameters to increase the yield of $\mathrm{Me}_{5}-\mathrm{GLY}$. First the DZM precursor was changed to $\mathrm{N}$ methyl- $N$-nitroso- $p$-toluenesulfonamide for its less vigorous reaction with alkaline solutions at room temperature and homogenous reaction conditions produces a more reliable amount of DZM. The product distribution of methylated species when using the sulfonamide precursor was found to favour the fully methylated Mes-GLY, but a modest amount of unmodified product was still observed during these trials. Second the analyte solvent was found to have a large effect in promoting the formation of fully iTrEnDi modified product. By solubilizing herbicide standards dissolved in $\mathrm{MeOH}$, rather than $\mathrm{H}_{2} \mathrm{O}$, the reaction between solvent and diazomethane could be decreased. The methylation of hydronium ions in acidic aqueous solution has been studied extensively and the 
methylation of $\mathrm{H}_{2} \mathrm{O}$ via protonated DZM decreases the overall methylation of an analyte in solution. ${ }^{24}$ The solubility of GLY in $\mathrm{MeOH}(231 \mathrm{mg} / \mathrm{L})$ is significantly lower than in $\mathrm{H}_{2} \mathrm{O}(10 \mathrm{~g} / \mathrm{L}){ }^{25}$ but for our purposes of identifying and quantifying GLY in small amounts in biological samples ( 4 mg/L or less), the solubility in $\mathrm{MeOH}$ was not problematic. Finally, by adding an additional aliquot of $\mathrm{HBF}_{4}$ midway through the reaction served to maintain a low $\mathrm{pH}$ to promote a final alkylation at nitrogen. Figure 5.4.3A shows the LCMS analysis of the iTrEnDi modified solution of GLY and AMPA using optimized conditions that produced less than $1 \%$ of undermethylated substrates (Figure S2). For the methylated compounds, a $\mathrm{R}_{\mathrm{T}} 1.5$ min later was found for AMPA and a 3.5 min later retention time was found for glyphosate, compared to that of unmethylated material. We were pleased to find optimized conditions worked remarkably well with both GLY and AMPA.

To be certain of the identity of m/z 182 highlighted in Figure 5.4.3A, a HRMS product ion spectrum was obtained and analyzed. The most abundant product ion observed in Figure $5.4 .3 \mathrm{~B}$ was $\mathrm{m} / \mathrm{z} 58$ can be identified as the loss of $\mathrm{C}_{3} \mathrm{H}_{9} \mathrm{OP}$ via a McLafferty rearrangement similar to Mes-GLY. We chose $\mathrm{m} / \mathrm{z} 182 \rightarrow 58$ and $182 \rightarrow 93$ to be the transitions for further MRM analysis based on their intensity in the $\mathrm{MS}^{2}$ spectrum. With optimal MRM transitions, the disagreeable elution profile of methylated AMPA could be addressed. Chromatographic mobile phase conditions were found to drastically influence elution profile of methylated APMA. Small changes to the composition of the initial loading solvent were used to improve the chromatographic resolution of Mes-AMPA (Figure 5.4.3C). Systematically changing the percentage of acetonitrile (ACN) during the first minute of the chromatographic gradient and observing the elution profile of methylated AMPA, $0.5 \% \mathrm{ACN}$ in $\mathrm{H}_{2} \mathrm{O}$ was determined give the best peak shape. With 
optimized reaction conditions to produce fully modified standards and chromatographic solvent composition in hand we could begin to determine the effect of methylation had on the analysis of GLY and AMPA compared to unmodified products.
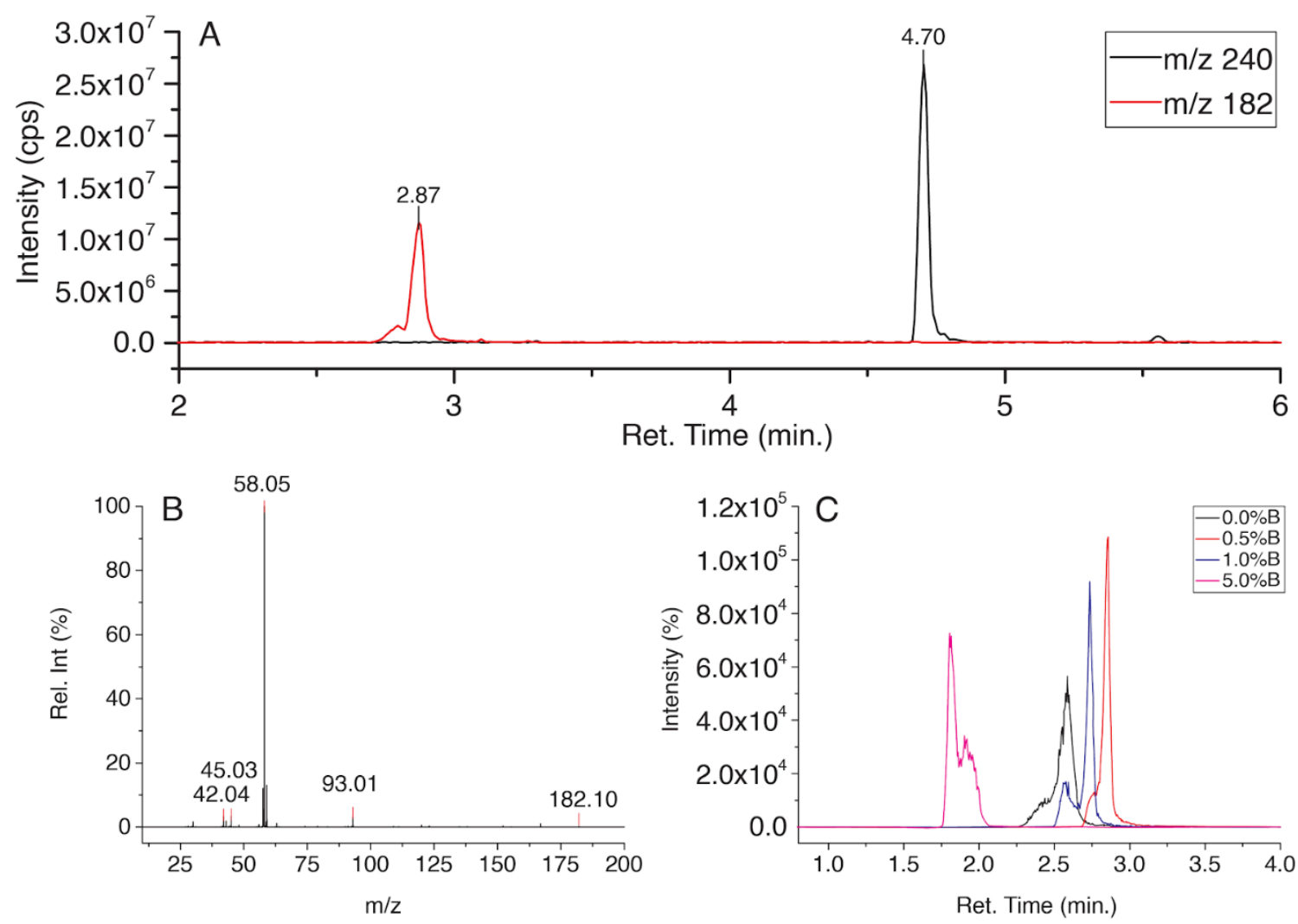

Figure 5.4.3 Full modification of glyphosate and AMPA with DZM using improved reaction conditions (A). Direct spray nESI MS ${ }^{2}$ analysis of Me-AMPA (B) and LC gradient optimization for peak shape of Mes-AMPA (C)

To accurately analyze the intensity differences between iTrEnDi modified and unmodified compounds we reviewed each $\mathrm{MS}^{2}$ parameter for to find the optimal conditions for fragmentation and MRM analysis. Table 5.3.1 shows the optimized parameters for both unmodified and iTrEnDi derivatized GLY and AMPA. Once unmethylated and DZM treated standards could be analyzed using MRM we could reliably compare them to 
observe the increase in peak area. The comparison was made using a lower concentration of modified analytes than unmodified due to saturation of the detector, and fold increases were extrapolated based on a linear relationship of modified standards. Figure 5.4.4(right) shows the overlaying single reaction monitoring (SRM) chromatographs of both derivatized and underivatized GLY and AMPA. Retention time of Me5-AMPA and Me5GLY were observed to increase by 1.5 and 3.0 min respectively. The effect of methylation on these compounds to retention in a C18 column is highly beneficial to the analysis of GLY and AMPA in complex matrices. The limit of detection (LOD) and limit of quantitation of each were estimated by the injection of diluted sample and determined to be concentration of analyte that give signal-to-noise $(\mathrm{S} / \mathrm{N})$ ratio of three and ten, respectively. For Mes-AMPA the LOD and LOQ were found to be $2.0 \mathrm{pmol}(18 \mathrm{ng} / \mathrm{L})$ and 2.5 pmol $(22 \mathrm{ng} / \mathrm{L})$. Furthermore, the approximate LOD and LOQ for Mes-GLY was determined to be $1.9 \mathrm{pmol}(22 \mathrm{ng} / \mathrm{L})$ and $2.0 \mathrm{pmol}(24 \mathrm{ng} / \mathrm{L})$. The LOD and LOQ of iTrEnDi modified analytes are significantly lower than that of other reported derivatization/reverse-phase LCMS strategies published to date.

Figure 5.4.4(left) shows the average normalized peak areas determined from a triplicate injection (unmodified) and a chemical triplicate (modified) and showcases the reproducibility. The reproducibility was determined by measuring the variance of three chemical triplicate reactions on a standard stock solution of GLY and AMPA. The peak area was normalized to caffeine as an internal standard, and the relative standard deviation was found to be $17 \%$ and $6 \%$ for derivatized AMPA and GLY, respectively. The signal increase due to modification with DZM, when dilution is considered, was calculated to be 339.7 (AMPA) and 12.4-fold (GLY). The values for normalized peak areas for this 
comparison can be found in Table S1. The large increase in MS signal after methylation will surely have a positive impact the confidence of detection and quantification of GLY and AMPA in environmental and toxicological studies.
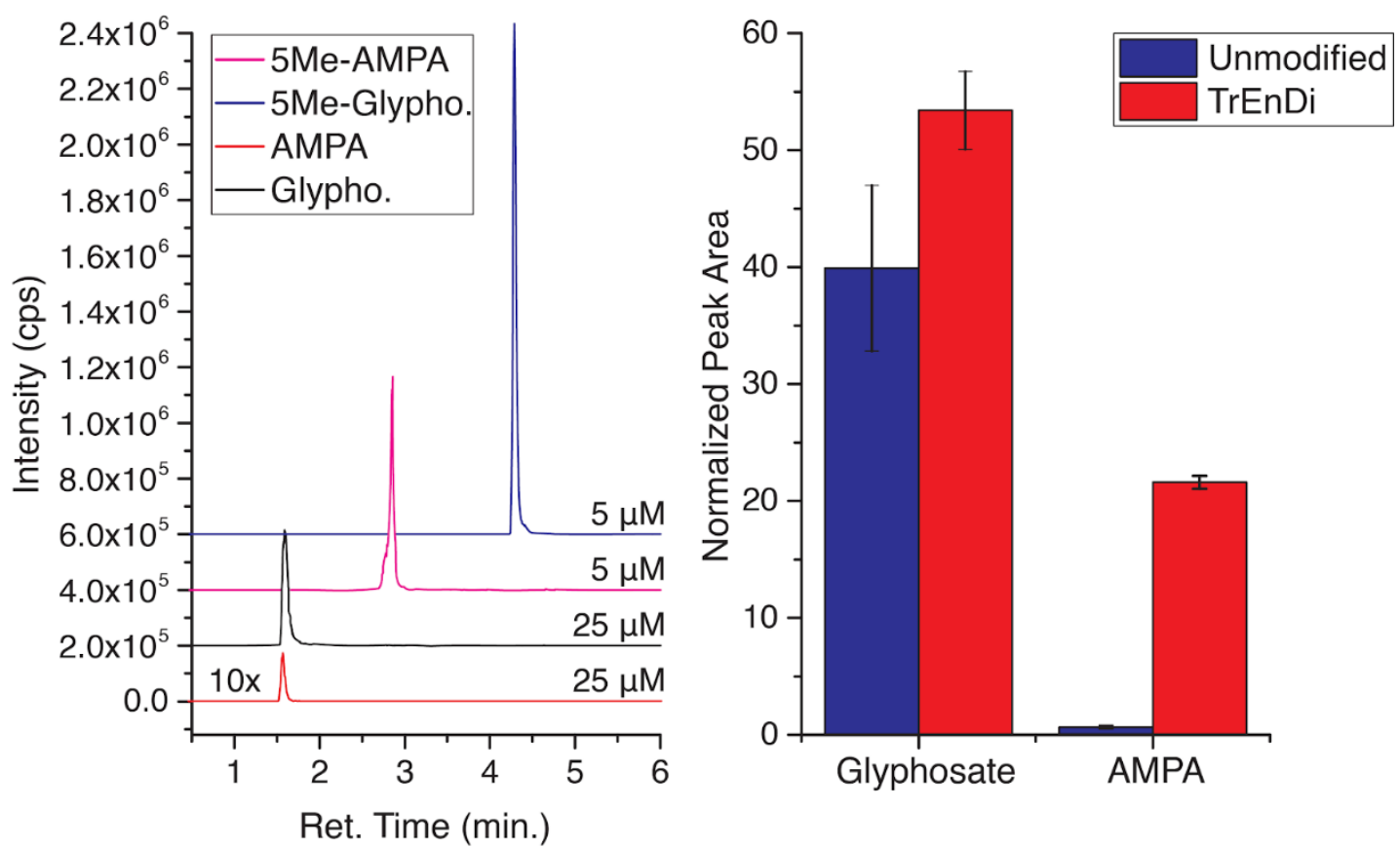

Figure 5.4.4 LCMS analysis of Glyphosate and AMPA with optimized multiple reaction monitoring parameters (left). Comparison of normalized peak area between TrEnDi modified $(5 \mu \mathrm{M})$ and unmodified $(25 \mu \mathrm{M})$ GLY and AMPA (right)

A calibration curve was constructed (Figure 5.4.5A) by plotting peak area of modified GLY and AMPA at a range of concentrations and fitting with a trendline. Methylated AMPA in the range of 0.13 to $5.00 \mu \mathrm{M}$ showed good linearity, measured by $\mathrm{R}^{2}$ of six different concentrations. However, methylated GLY had a slightly lower linearity in the range of 0.10 to $5.00 \mu \mathrm{M}$ likely due to an erroneous injection. Given more time to accomplish an accurate and precise calibration curve each concentration would be injected in triplicate and the peak area would be normalized to an internal standard. Following the 
creation of a standard curve we opted to test our novel derivatization strategy on samples with more complex matrices. Figure 5.5.5B shows the intensity of the quantifier and qualifier transitions used for the MRM analysis of methylated analytes found in diluted Roundup solution and an aqueous extraction performed on soybean that were known to have been treated with GLY containing herbicides. Soybean samples were sprayed twice, approximately two and one months prior to analyzing the soybean plant sample. Furthermore, the soybean plant was grown in an outdoor environment using the manufacturer's instructions, was subjected to weather from environmental conditions in Ottawa, Canada over the summer months. Both Roundup and soybean extract samples confidently showed the presence of Me5-GLY in varying amounts, but the presence of MesAMPA was uncertain due to the presence of some low molecular weight interference and low intensity of qualifier ions. Using the calibration curve, we could determine a concentration of $9 \mathrm{pmol}(110 \mathrm{ng} / \mathrm{L})$ of modified GLY in the soybean extract. This amount of GLY found from a biological source is unprecedented, where most studies could only detect concentrations above $200 \mathrm{ng} / \mathrm{L}$. 

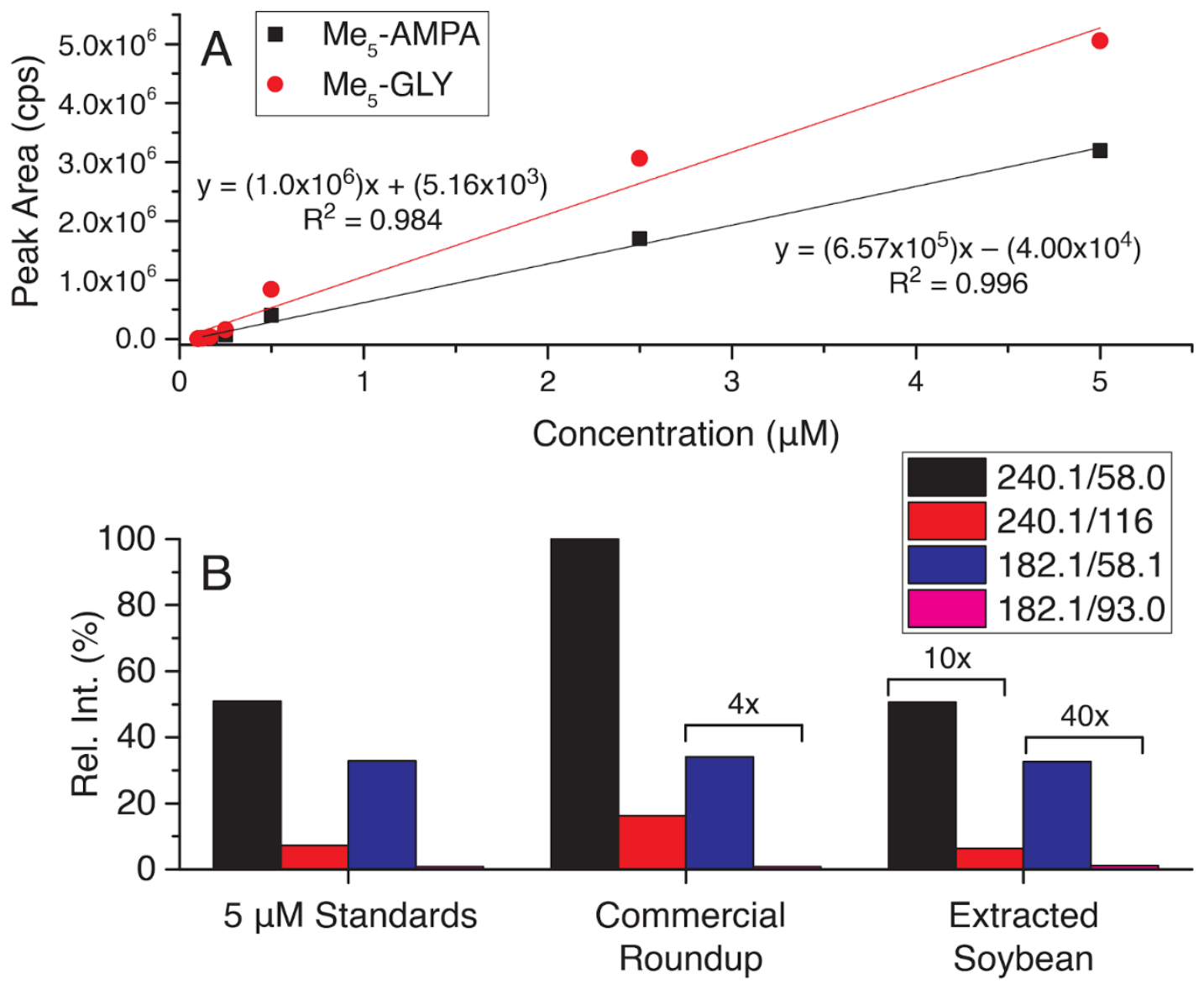

Figure 5.4.5 Calibration curve prepared for iTrEnDi derivatized GLY and AMPA

(A) LCMS analysis of iTrEnDi modified herbicide from commercial Roundup ${ }^{\circledR}$ and soybean extract with optimized multiple reaction monitoring parameters (B). Values for Me5-GLY and -AMPA are multiplied by indicated factor to help visualization

\subsection{Conclusion}

In summary we have designed a novel method of chemical derivatization of the difficult to analyze herbicide GLY and its major degradation product AMPA. The process of methylation of analytes uses the iTrEnDi apparatus for the safe handling of DZM, where analytes are reacted with small amounts of gaseous diazoalkane to produce fixed positively 
charged species. Permethylated GLY and AMPA show improved chromatographic characteristics compared to unmodified counterparts when conventional C18 solid phase and $\mathrm{H}_{2} \mathrm{O} / \mathrm{MeCN}$ mobile phase are used. Considering the fixed positive charge produced during the iTrEnDi reaction, integration of this technique can be readily incorporated into the analysis of other pesticides workflows giving a more streamlined approach to the regulation of GLY and AMPA. Furthermore, sensitivity of methylated GLY and AMPA were found to be increased drastically over unmodified compounds and detection limits were observed in the low ng/L range. The unprecedented limit of detection for GLY and AMPA derivatives will allow for the greater understanding of their biological impacts to human health. Finally, an aqueous extract of soybeans treated with GLY containing pesticides was analyzed via derivatization with iTrEnDi and LCMS analysis successfully despite being exposed to the environment truly shows that our methodology is well equipped to deal with real world samples.

\subsection{References}

(1) De María, N.; Becerril, J. M.; García-Plazaola, J. I.; Hernández, A.; De Felipe, M. R.; Fernández-Pascual, M. J. Agric. Food Chem. 2006, 54 (7), 2621-2628.

(2) Valle, A. L.; Mello, F. C. C.; Alves-Balvedi, R. P.; Rodrigues, L. P.; Goulart, L. R. Environ. Chem. Lett. 2019, 17 (1), 291-317.

(3) Hébert, M. P.; Fugère, V.; Gonzalez, A. Front. Ecol. Environ. 2019, 17 (1), 48-56.

(4) Reddy, K. N.; Rimando, A. M.; Duke, S. O. J. Agric. Food Chem. 2004, 52 (16), $5139-5143$.

(5) González-Martínez, M. Á.; Brun, E. M.; Puchades, R.; Maquieira, Á.; Ramsey, K.; 
Rubio, F. Anal. Chem. 2005, 77 (13), 4219-4227.

(6) Okada, E.; Coggan, T.; Anumol, T.; Clarke, B.; Allinson, G. Anal. Bioanal. Chem. 2019, 411 (3), 715-724.

(7) Carretta, L.; Cardinali, A.; Marotta, E.; Zanin, G.; Masin, R. J. Chromatogr. A 2019, $1600,65-72$.

(8) Williams, G. M.; Kroes, R.; Munro, I. C. Regul. Toxicol. Pharmacol. 2000, 31 (2 I), $117-165$.

(9) Marc, J.; Mulner-Lorillon, O.; Bellé, R. Biol. Cell 2004, 96 (3), 245-249.

(10) Weeks Santos, S.; Gonzalez, P.; Cormier, B.; Mazzella, N.; Bonnaud, B.; Morin, S.; Clérandeau, C.; Morin, B.; Cachot, J. Aquat. Toxicol. 2019, 216 (February), 105291.

(11) Chen, D.; Miao, H.; Zhao, Y.; Wu, Y. J. Chromatogr. A 2019, 1587, 73-78.

(12) Steinborn, A.; Alder, L.; Michalski, B.; Zomer, P.; Bendig, P.; Martinez, S. A.; Mol, H. G. J.; Class, T. J.; Costa Pinheiro, N. J. Agric. Food Chem. 2016, 64 (6), 14141421.

(13) Pinto, E.; Soares, A. G.; Ferreira, I. M. P. L. V. O. Anal. Methods 2018, 10 (5), 554561.

(14) Wasslen, K. V.; Tan, L. H.; Manthorpe, J. M.; Smith, J. C. Anal. Chem. 2014, 86 (7), 3291-3299.

(15) Wasslen, K. V.; Canez, C. R.; Lee, H.; Manthorpe, J. M.; Smith, J. C. Anal. Chem. 2014, 86 (19), 9523-9532.

(16) Canez, C. R.; Shields, S. W. J.; Bugno, M.; Wasslen, K. V.; Weinert, H. P.; Willmore, W. G.; Manthorpe, J. M.; Smith, J. C. Anal. Chem. 2016, 88 (14), 69967004. 
(17) de Boer, J.; Backer, H. J. Org. Synth. 2003, No. September, 96-96.

(18) Braun, C. E.; Cook, C. D.; Charles, Merritt, J.; Rousseau, J. E. Org. Synth. 1951, 31 (September), 77.

(19) Glass, R. L. J. Agric. Food Chem. 1984, 32 (6), 1249-1253.

(20) Seyed Khademi, S. M.; Telgheder, U.; Valadbeigi, Y.; Ilbeigi, V.; Tabrizchi, M. Int. J. Mass Spectrom. 2019, 442, 29-34.

(21) Guo, H.; Gao, Y.; Guo, D.; Liu, W.; Wang, J.; Zheng, J.; Zhong, J.; Zhao, Q. SN Appl. Sci. 2019, 1 (4), 1-8.

(22) Coutinho, C. F. B.; Coutinho, L. F. M.; Mazo, L. H.; Nixdorf, S. L.; Camara, C. A. P. J. Chromatogr. A 2008, 1208 (1-2), 246-249.

(23) Oliveira Pereira, E. A.; Freitas Melo, V.; Abate, G.; Masini, J. C. Anal. Bioanal. Chem. 2019, 411 (11), 2317-2326.

(24) McGarrity, J. F.; Smyth, T. J. Am. Chem. Soc. 1980, 102 (24), 7303-7308.

(25) MacLachlan, D. Glyphosate http://www.fao.org/tempref/docrep/fao/009/a0439e/a0439e18.pdf (accessed Nov 12, 2019). 


\subsection{Supporting Information}

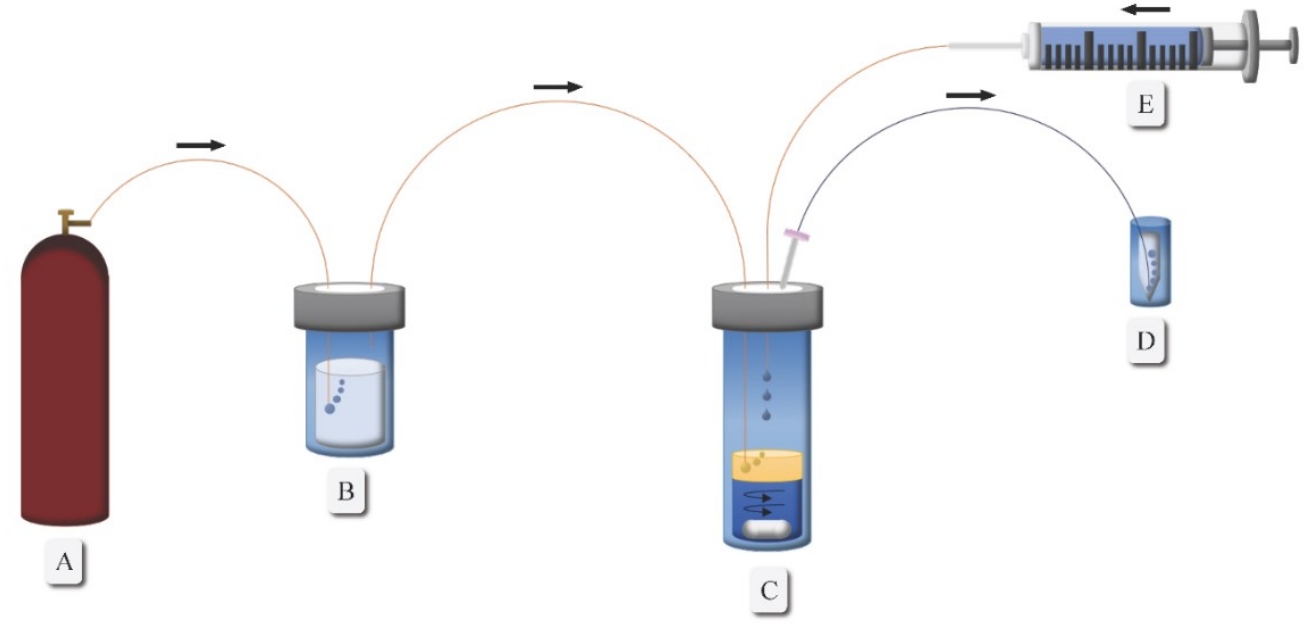

Figure 5.7.1 (S1) Diagram of the experimental setup of iTrEnDi derivatization method. A) $\mathrm{N}_{2}$ gas source. B) diethyl ether- $\mathrm{N}_{2}$ formation vial. C) Diazoalkane reaction chamber $\left(\mathrm{H}_{2} \mathrm{O}, \mathrm{NMU}\right.$, diethyl ether) or (diethylene glyocol monoethyl ether, $N$-methyl- $N$-nitroso-p-toluenesulfonamide, diethyl ether) D) Analyte vial (glycerophospholipids/sphingolipids/small molecules) E) Base solution (6.1 M KOH in $\left.\mathrm{H}_{2} \mathrm{O}\right)$. 


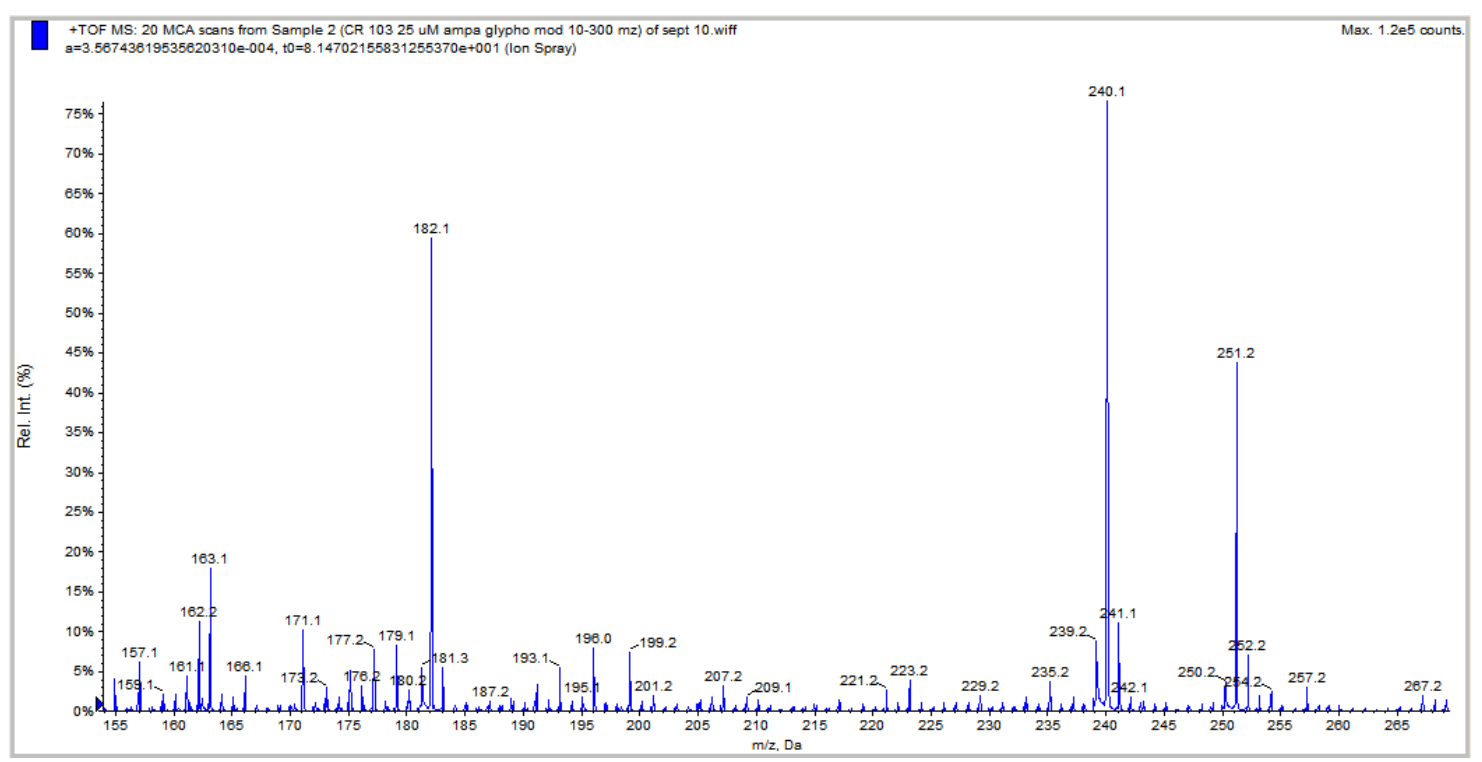

Figure 5.7.2 (S2) nESI in +MS of iTrEnDi modified GLY and AMPA using optimized reaction conditions showing no unmethylated or undermethylated reaction products.

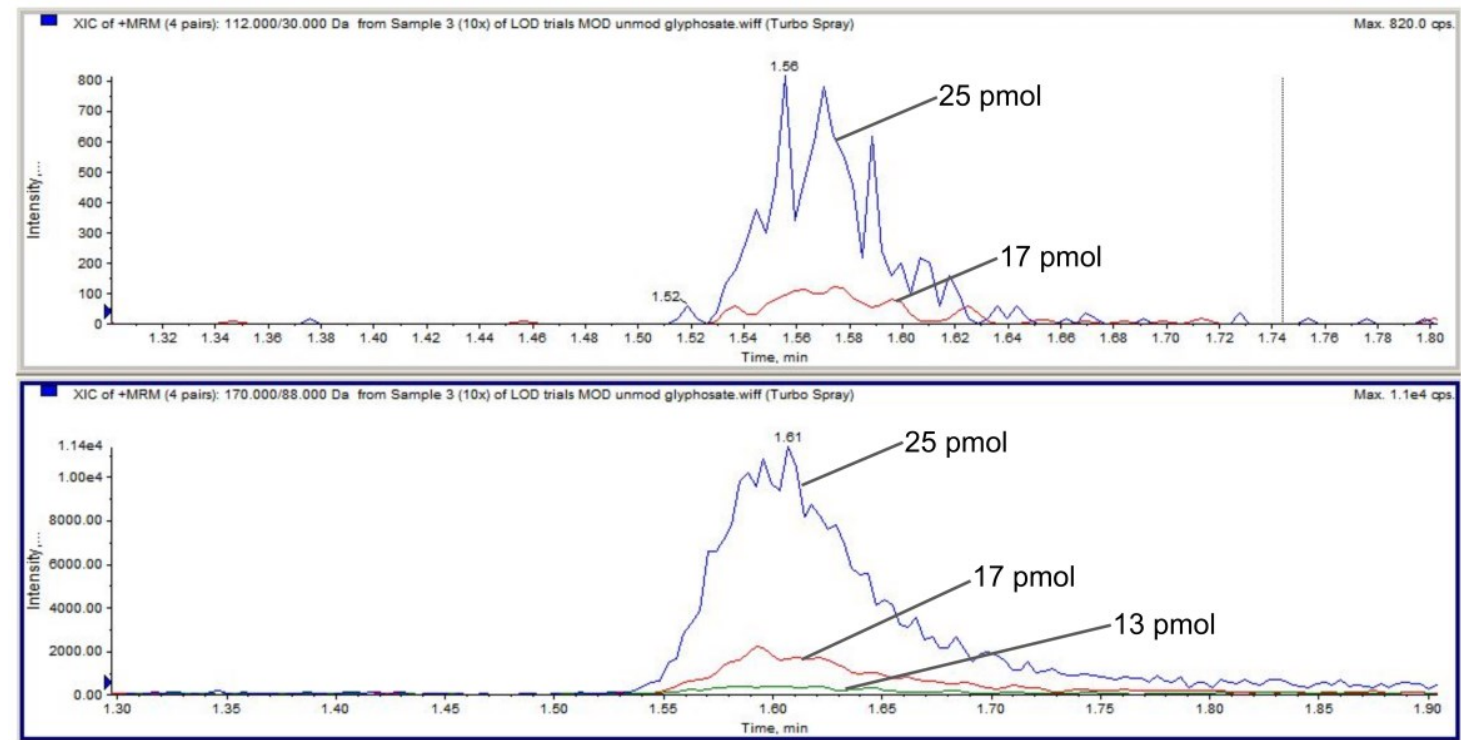

Figure 5.7.3 (S3) Sensitivity of unmodified AMPA (top) and GLY (bottom). 


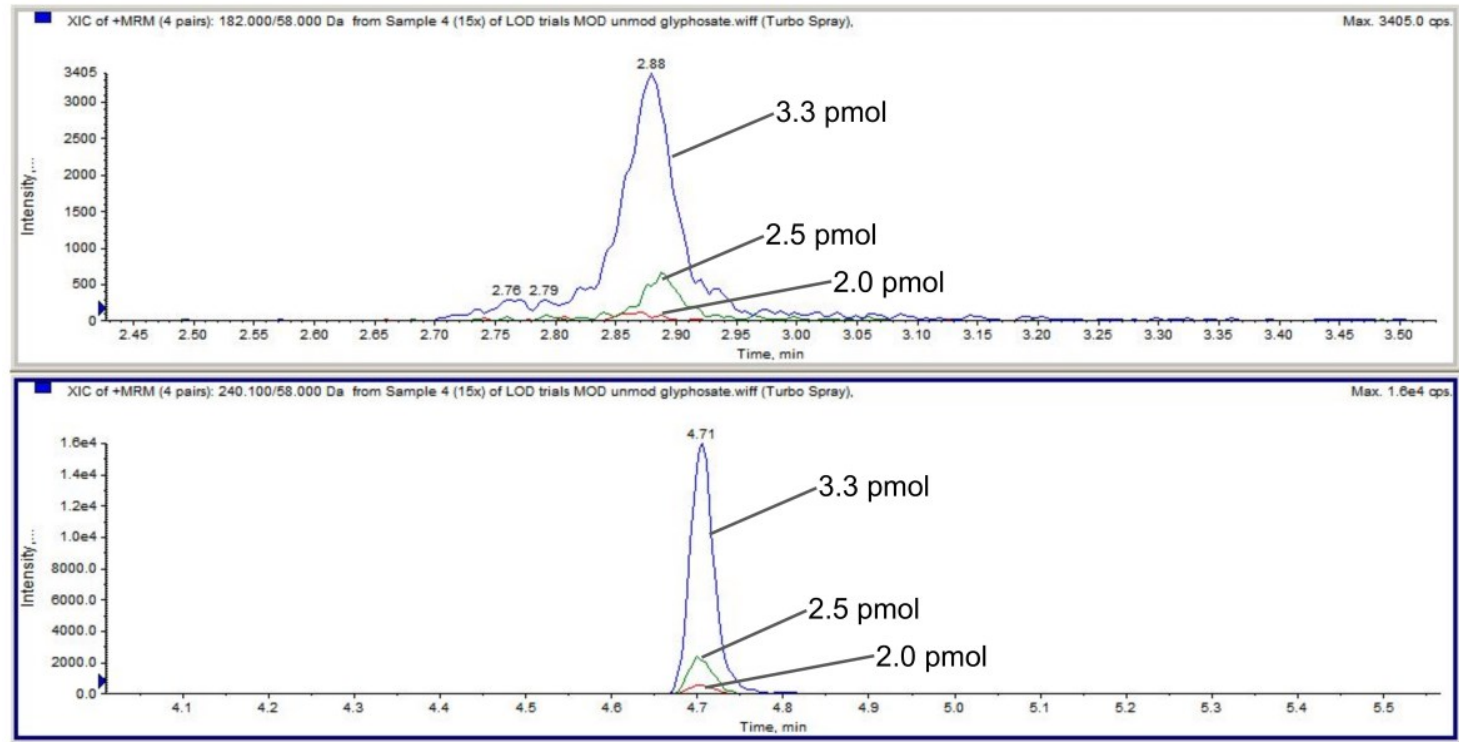

Figure 5.7.4 (S4) Sensitivity of derivatized AMPA (top) and GLY (bottom). 
Table 5.7.1 (S1) Sensitivity enhancements of iTrEnDI modification of GLY and

AMPA.

\begin{tabular}{|c|c|c|c|c|c|c|}
\hline & & $\mathrm{k}$ Area (cp & & & Normali & ak Area \\
\hline & AMPA & GLY. & Caffeine & & AMPA & GLY \\
\hline & $4.70 \mathrm{E}+06$ & $5.44 \mathrm{E}+06$ & $1.03 \mathrm{E}+05$ & & 45.50 & 52.70 \\
\hline & $3.19 \mathrm{E}+06$ & $5.05 \mathrm{E}+06$ & $9.99 \mathrm{E}+04$ & & 32.00 & 50.50 \\
\hline & $1.67 \mathrm{E}+06$ & $2.44 \mathrm{E}+06$ & $1.02 \mathrm{E}+05$ & & 42.20 & 57.00 \\
\hline & & & & Average & 39.90 & 53.40 \\
\hline $\begin{array}{c}\text { 11 rEnD1 } \\
(5 \mu \mathrm{M})\end{array}$ & & & & $\begin{array}{l}\text { Standard } \\
\text { Deviation }\end{array}$ & 7.08 & 3.32 \\
\hline & & & & $\begin{array}{c}\text { Relative } \\
\text { Standard } \\
\text { Deviation } \\
(\%)\end{array}$ & 17.74 & 6.21 \\
\hline & $6.00 \mathrm{E}+04$ & $2.18 \mathrm{E}+06$ & $1.03 \mathrm{E}+05$ & & 0.58 & 21.10 \\
\hline & $5.86 \mathrm{E}+04$ & $2.16 \mathrm{E}+06$ & $1.01 \mathrm{E}+05$ & & 0.58 & 21.40 \\
\hline & $6.11 \mathrm{E}+04$ & $2.25 \mathrm{E}+06$ & $1.02 \mathrm{E}+05$ & & 0.60 & 22.20 \\
\hline & & & & Average & 0.59 & 21.60 \\
\hline $\begin{array}{l}\text { Unmodified } \\
(25 \mu \mathrm{M})\end{array}$ & & & & $\begin{array}{l}\text { Standard } \\
\text { Deviation }\end{array}$ & 0.01 & 0.55 \\
\hline & & & & $\begin{array}{c}\text { Relative } \\
\text { Standard } \\
\text { Deviation } \\
\quad(\%)\end{array}$ & 2.06 & 2.54 \\
\hline Fold Change & & & & & $339.68 x$ & $12.39 x$ \\
\hline
\end{tabular}




\section{Chapter 6: Solution-Phase Charge Inversion of Glycerophospholipids via complex formation with a spectrum of Diacid Structures}

\subsection{Introduction}

The fatty acyl components of lipids are highly variable across the different lipid classes and play an important role in their bioactivity. The identification of fatty acyl chains of lipids from a biological sample are commonly performed by ESI-MS in negative ion mode via CID. However, phospholipids that are subjected to the TrEnDi protocol are given a fixed positive charge, and thus can only be observed in positive ion mode. Fragmentation of $\mathrm{PC}$ and $\mathrm{PE}$ in positive ion mode, gives cleavage of the polar head group rather than fatty acyl substituents. Recently, the McLuckey group at Perdue was able to induce a charge inversion (+ to -) of PE and PC via a gas phase complexation with the dicarboxylic acid (DCA) 1,4-phenylenedipropionic acid (PDPA) (Scheme 6.1.1). ${ }^{1,2}$ In this ion/ion reaction, the PC is ionized via ESI-MS in positive ion mode, and held in an ion trap (another type of mass analyzer) while deprotonated PDPA is ionized by ESI-MS in negative ion mode. The ionized PC and PDPA are allowed to interact within the ion trap yielding an overall negatively charged adduct, before MS and MS/MS analysis. While, the McLuckey procedure requires the use of specialized equipment, this research will seek to develop methodology that is suitable in solution with the TrEnDi modified lipids with no instrument modification necessary. Solution-phase charge inversion, in combination with TrEnDi, could not only increase sensitivity but also enable us to obtain invaluable structural information from glycerophospholipids. 


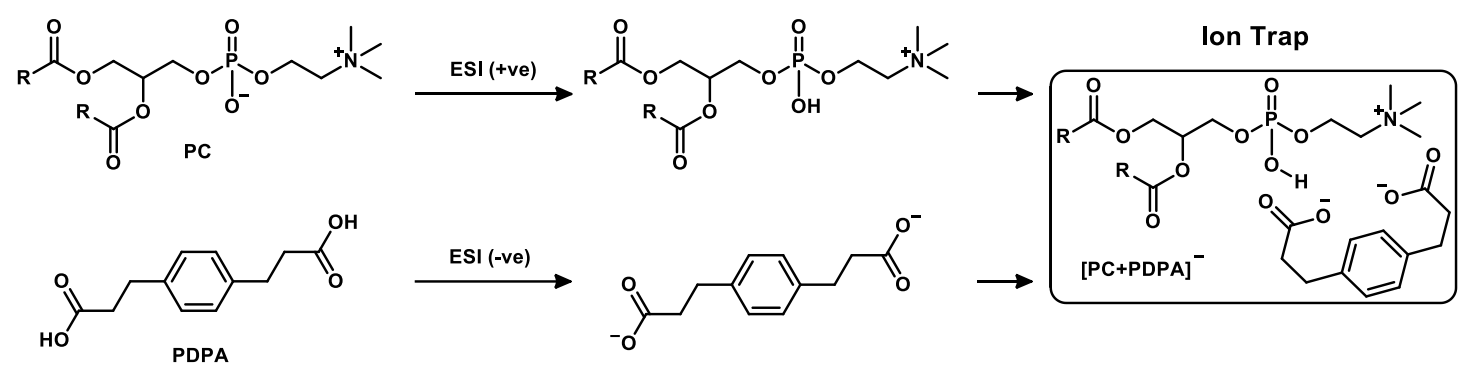

Scheme 6.1.1 The charge inversion of PC using PDPA in an ion trap

\subsection{Overview}

This section details the investigation of four different diacids (Figure 6.2.1), for their activity towards forming net negative complexes with TrEnDi modified PC(14:0/18:1) standard. When possible, a product ion scan will be performed on the diacid/PC complex to observe product ions indicative of fatty acids. Once a suitable diacid is found, a comparison to standard techniques for fatty acyl fragmentation will be made, using an external standard.<smiles>O=S(=O)([O-])[O-]</smiles>

$$
\begin{aligned}
& \text { Bistetrabutyl } \\
& \text { ammonium } \\
& \text { sulfate } \\
& \text { (TBAS) }
\end{aligned}
$$<smiles>O=C(O)C(=O)O</smiles>

$$
\text { Oxalic Acid }
$$$$
\text { (OA) }
$$<smiles>O=C(O)CCSCCC(=O)O</smiles>

Thiodipropionic Acid (TDPPA)<smiles>O=C(O)COc1cccc(OCC(=O)O)c1</smiles>

Resorcinol-0,0'diacetic acid (RDAA)

Figure 6.2.1 Four diacids to be investigated via solution phase charge inversion of 


\subsection{Experimental}

Chemicals and Materials. PC (18:1/ 14:0) was purchased from Avanti Polar Lipids Inc. (Alabaster, AL, USA) as a solution in chloroform. Resorcinol, chloroacetic acid, thiodipropionic acid, tetrabutylammonium hydroxide and tetrabutylammonium bisulfate were purchased from Sigma-Aldrich (St. Louis, MO, USA). Diethylether, ethanol, methanol, acetonitrile and isopropanol, were purchased from Caledon Laboratories Ltd. (Georgetown, ON, Canada). Ammonium hydroxide (28\%) and ammonium acetate was purchased from Anachemia Canada Inc. (Montreal, QC, Canada) Dr Jeff Manthorpe previously synthesized Diazald earlier this year according to the procedure by deBoer and Backer. $^{3}$

Preparation of Diazomethane. Warning! All handling of diazomethane solutions were carried out behind a blast shield and in a fume hood. The production of diazomethane was carried out in the Aldrich Mini Diazald generator (clear seal joints) following the procedure in Aldrich's technical bulletin for diazomethane. ${ }^{4}$ Diazald (5 g) was dissolved in ether (45 $\mathrm{ml})$ in the upper dropping funnel of the apparatus. Then $\mathrm{KOH}(5 \mathrm{~g})$ was dissolved in water $(8 \mathrm{ml})$ and $\mathrm{EtOH}(10 \mathrm{ml}, 95 \%)$ in the lower reaction chamber of the DZM generator. The caustic solution was heated to $65^{\circ} \mathrm{C}$ in an oil bath. The cold finger and receiving flask were cooled down to $-78{ }^{\circ} \mathrm{C}$ using dry ice pellets and isopropanol. The yellow diazald solution was added dropwise to the $\mathrm{KOH}$ solution at such a rate to have one drop of diazomethane solution for every 3 drops of diazald solution. Once the reaction was completed, ethereal diazomethane was kept in a closed shock insulating container at $-15{ }^{\circ} \mathrm{C}$. The solution can be stored up to 3 weeks at this temperature. 
A working solution of $1000 \mu \mathrm{M}$ solution of PC (18:1/ 14:0) was prepared in ethanol from commercial chloroform solution. A $400 \mu \mathrm{M}$ lipid solution in $25 \mu$ l of ethanol was prepared in a clear vial from the working $1000 \mu \mathrm{M}$ stock. To the lipid solution, $1 \mu \mathrm{l}$ of a $\mathrm{Et}_{2} \mathrm{O}: \mathrm{HBF}_{4}(14: 1)$ was added before the dropwise addition of ethereal diazomethane using a fire polished glass pipette. The diazomethane was added until a light-yellow colour persisted for 30 seconds $(\sim 0.3-0.5 \mathrm{ml})$. After the diazomethane was added, the solvents were evaporated under a steady stream of nitrogen gas for $5 \mathrm{~min}$. The methylated lipid was re-suspended in $100 \mu \mathrm{l}$ of ethanol (giving $100 \mu \mathrm{M}$ concentration), capped and stored in the freezer under an inert atmosphere. (This procedure was adapted from Smith et. al. ${ }^{5}$

Preparation of diacid solutions. Diacid $(0.15 \mathrm{mmol})$ was dissolved in $\mathrm{MeOH}: \mathrm{MeCN}(1: 1$, $14.85 \mathrm{ml})$. Then $150 \mu \mathrm{l}$ of ammonium hydroxide (28\%) was added carefully. Ammonium diacid solution was stirred overnight.

A $0.5 \mathrm{M}$ solution of bis(tetrabutylammonium) sulfate (TBAS) was prepared by suspending tetrabutylammonium bisulfate $(1.69 \mathrm{~g})$ in water $(6.66 \mathrm{ml})$. To this suspension tetrabutylammonium hydroxide $(3.33 \mathrm{ml}, 40 \%$ wt. in water) was added. The TBAS solution was diluted to $10 \mathrm{mM}$ in ethanol.

Lipid solution preparation. Lipids $(50 \mu 1)$ were dissolved in one of the diacid solutions at a concentration of $100 \mu \mathrm{M}$ with a $5 \mu \mathrm{l}$ aliquot of $\mathrm{NaBPh}_{4}$ solution $(10 \mu \mathrm{M})$ as an external standard. For the diacid solutions that had poor solubility a $1 \mathrm{ml}$ aliquot of the mixture was placed into a $1 \mathrm{ml}$ centrifuge vial and were centrifuged at $5 \mathrm{xg}$ for $5 \mathrm{~min}$. Then the supernatant was used to dissolve the modified lipid.

Synthesis of Resorcinol-O,O'-diacetic acid. Resorcinol (5.5 g) and chloroacetic acid (10.5 g) were added successively to a room temperature solution of $\mathrm{NaOH}(10.1 \mathrm{~g})$ in water 
$(100 \mathrm{ml})$ to give a dark brown solution. The flask was fitted with a condenser and heated to reflux in an oil bath for $4 \mathrm{~h}$. The reaction was cooled before being acidified with aqueous $\mathrm{HCl}(4 \mathrm{M}, 50 \mathrm{ml})$ to a $\mathrm{pH}$ of 2 . The mixture was allowed to stand for $30 \mathrm{~min}$ before filtering off a yellow solid $(7 \mathrm{~g})$. The crude solid was dissolved in hot water $(80 \mathrm{ml})$ and cooled to room temperature to form colorless needles. The crystallization was performed twice more to obtain pure RDAA. This procedure was adapted from Zhu et. al. ${ }^{6}$

${ }^{1} \mathrm{H}$ NMR ( $\left(d_{6}\right.$-DMSO, $\left.300 \mathrm{MHz}\right): \delta 7.18\left(\mathrm{t},{ }^{3} J=8.1,1 \mathrm{H}\right), 6.49\left(\mathrm{dd},{ }^{3} J=8.1,2.4,2 \mathrm{H}\right), 6.46$ (t, $\left.{ }^{3} J=2.4,1 \mathrm{H}\right), 4.64$ (s, 4H).

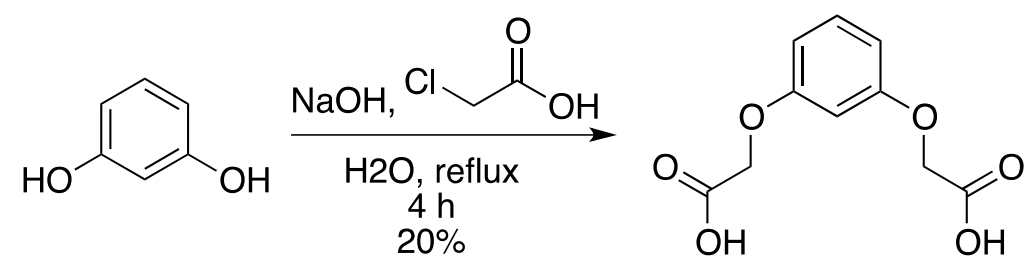

\section{Scheme 6.3.1 Synthesis of RDAA}

ESI-MS and MS/MS of modified and unmodified lipids. Six $\mu 1$ of the lipid solution was injected into a Proxeon nanoelectrospray emitter (Thermo Scientific, Odense, Denmark). The solution was analysed directly using a Sciex QStar XL hybrid quadrupole time of flight mass spectrometer fitted with a nano-ESI source (Sciex, Framingham, MA, USA). Spectra were obtained using capillary voltages of 1000 to $1100 \mathrm{~V}$ for positive ion mode and -700 to $-850 \mathrm{~V}$ for negative ion mode. Declustering potential and focusing potentials used were set to 60 and $120 \mathrm{~V}$, respectively (the inverse polarity was used for negative ion mode). Collision energy for MS/MS experiments ranged from -30 to $-50 \mathrm{eV}$ having the collision gas pressure set at 4 units. 


\subsection{Results and Discussion}

The phosphatidylcholine lipid class is generally analyzed in positive ion mode with the addition of a proton source. The PC of interest has an exact mass of 731.55 Da and the protonated mass should therefore be $732.55 \mathrm{Da}$. To check the quality of our lipid standard, MS and MS/MS experiments in positive ion mode were performed (Figures 8 and 9). Peaks for both protonated $(\mathrm{M}+1)$ and sodiated $(\mathrm{M}+23)$ appeared quite readily. Also, by performing a product ion scan, we could readily generate the well-known fragment of 184 Da that is indicative of a PC being present (Figure 6.4.2). From these results, the lipid standard was determined to be pure and at a significant concentration to be observed using the QStar XL.

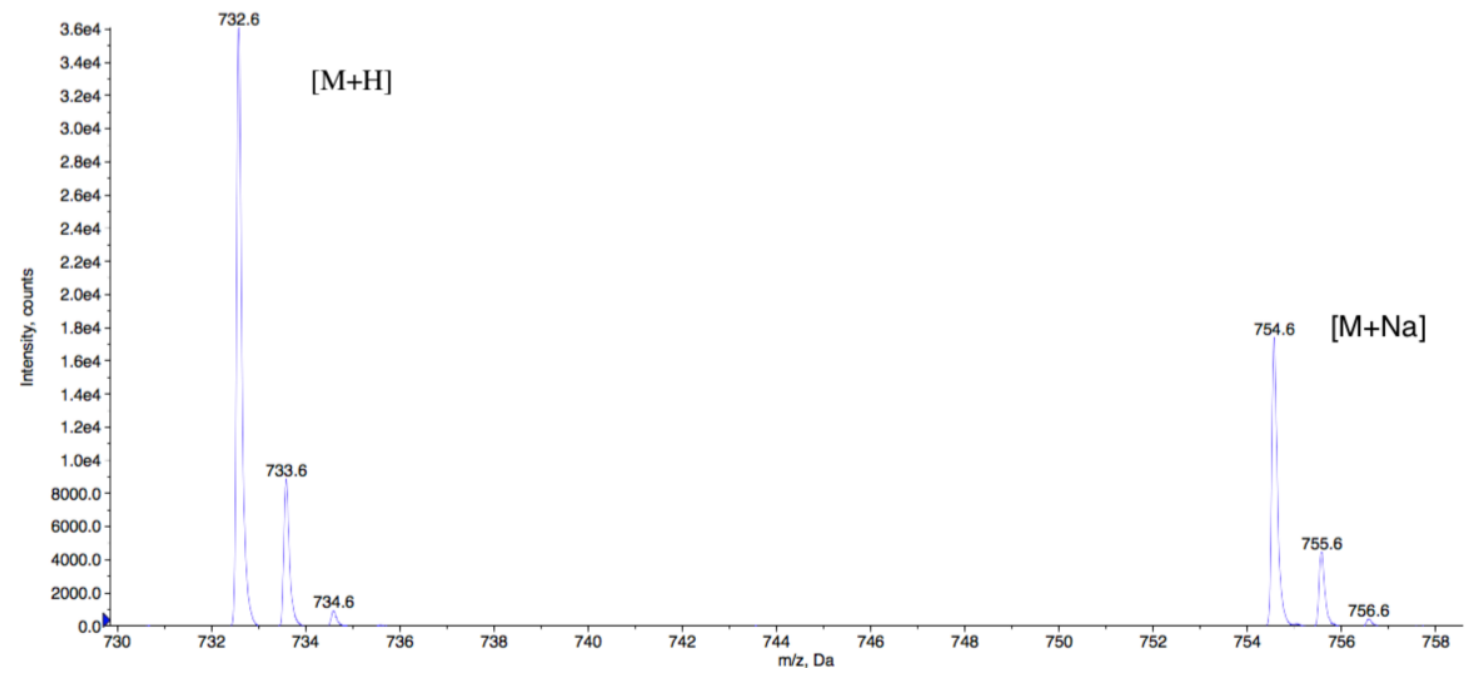

Figure 6.4.1 nESI-MS positive spectrum of $\mu \mathrm{M}$ PC with $10 \mathrm{mM} \mathrm{NH}_{4} \mathrm{OAc}$ in ethanol showing protonated and sodiated adducts 


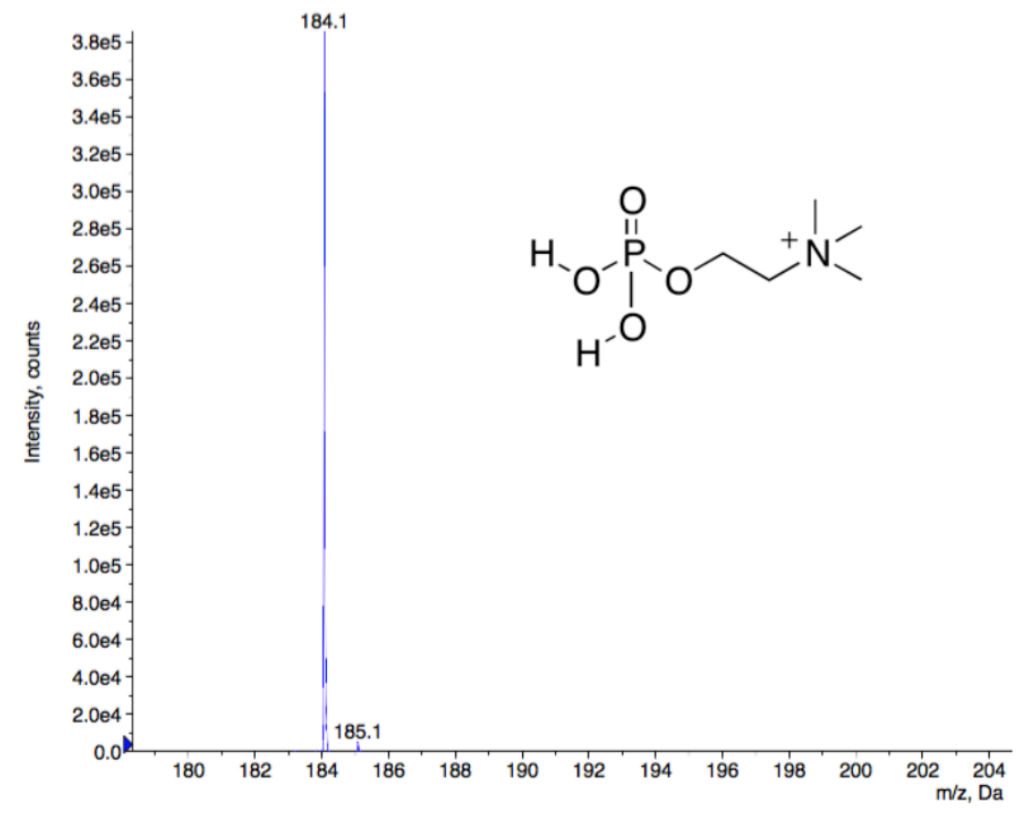

\section{Figure 6.4.2 nESI-MS ${ }^{2}$ positive product ion spectrum of $\mathrm{m} / \mathrm{z} 732.6$}

The next objective of this research is to modify the PC standard using the TrEnDi protocol and analyse the products by MS and MS/MS in positive ion mode. Methylation of PCs requires the use of a strong acid to first fully protonate the phosphoric acid moiety of the polar head group. The phosphoric acid functionality has an approximate pKa of 2 when protonated, and thus the use of $\mathrm{HBF}_{4}(\mathrm{pKa} \sim-0.4)$ is enough to have the reaction go to completion. Once methylated an addition of $15 \mathrm{Da}$ should be added to the parent $\mathrm{PC}$ and head group fragmentation. The lipid was observed without the use of a significant proton source due to the fact that after methylation it has a fixed positive charge and the formation of an ion is no longer reliant on proton affinity. Fragmentation of the peak at 746.6 Da gives us cleavage of the polar head group with the addition of $15 \mathrm{Da}$ corresponding to one methyl group (Figure 6.4.4). Each experiment to follow checks the viability of the lipid in positive ion mode in this way before running experiments in negative ion mode to asses the efficiency of methylation. 


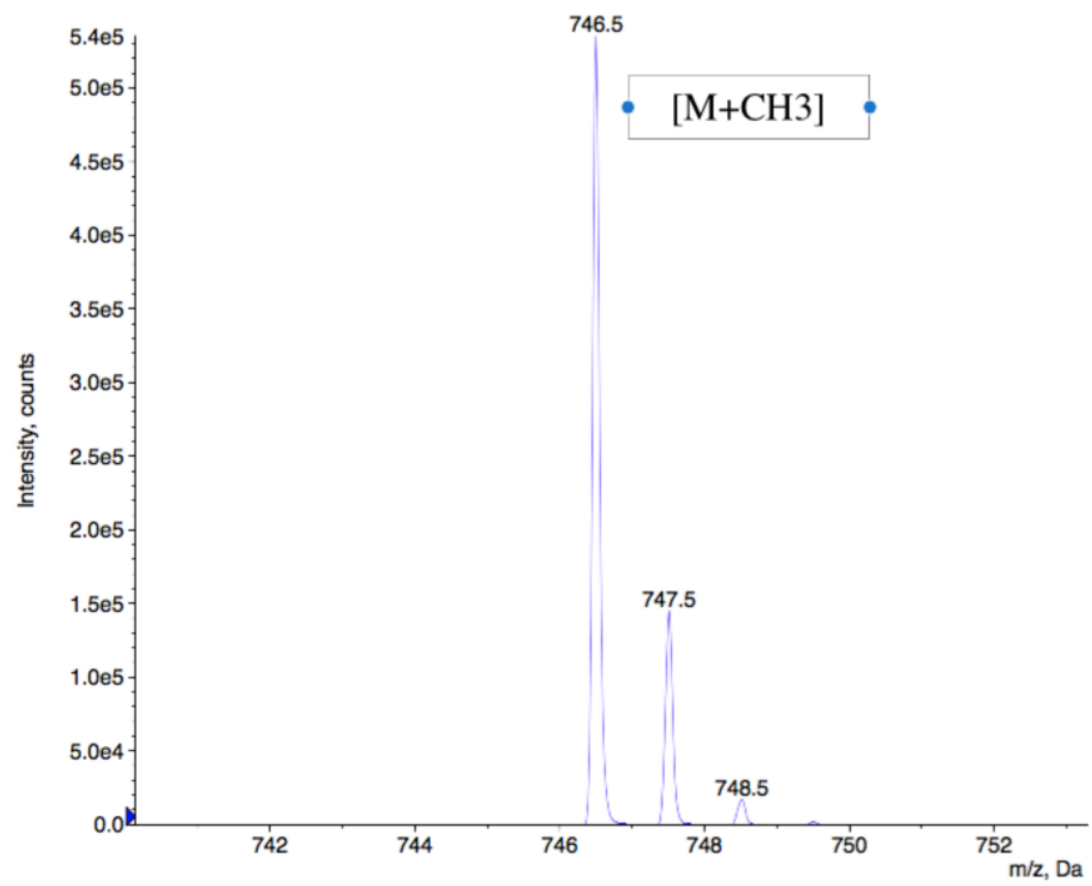

Figure 6.4.3 nESI-MS positive spectrum of TrEnDi modified $50 \mathrm{mM} \mathrm{PC}$ in EtOH

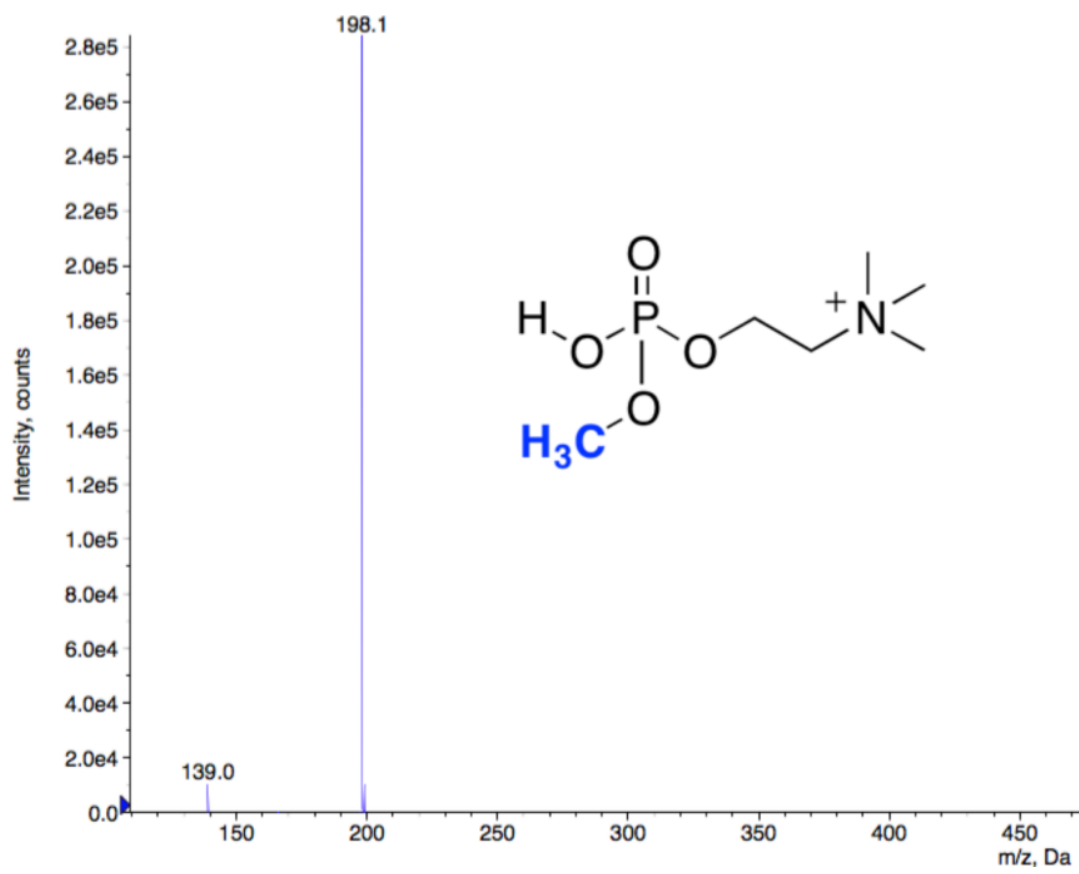

Figure 6.4.4 nESI-MS ${ }^{2}$ positive product ion spectrum of $\mathrm{m} / \mathrm{z} 746.5$ 
With the modified lipid in hand we could then start to investigate a range of diacids for the solution-phase charge inversion. The first diacid that was the most readily available was oxalic acid. At first a solution of sodium oxylate was prepared in a solution of water and ethanol (2:8) at $\sim 10 \mathrm{mM}$ and used in the ESI-MS of the modified lipid $(50 \mu \mathrm{M})$ with no positive results. At this point we re-analyzed the literature from the McLuckey group to find how their doubly deprotonated acid was introduced into the instrument. After some literature research two critical points of information were obtained. First the dicarboxylic acid was prepared in a solution of $\mathrm{MeOH}: \mathrm{MeCN}: \mathrm{NH}_{4} \mathrm{OH}$ (49.5:49.5:1 ratio), and secondly their lipid was in much larger concentrations for solution phase trials. Thus, we analyzed each of the acids mentioned above (Figure7) for their capability of adduct formation with the modified lipid using the new solvent system as well as doubling the concentration of lipid in solution.

Table 6.4.1 describes the results of each dianion used to create a charge inversion of the methylated lipid. The rational behind the four diacids used in the following experiments was based on their size, $\mathrm{pKa}$ and similarity to PDPA. The first dianion to be tried was the sulfate dianion with an organic soluble cation $(\mathrm{NBu} 4)$; due to its small size and low pka values this anion was a good candidate. However, no adduct with the modified lipid was ever observed in negative ion modes. The second and fourth dianion, oxylate and RDAA also yielded no adduct formation, possibly due to their poor solubility. But thiodi propionic acid (TDPPA) did form a complex with the modified lipid to give a peak at 922.6 $\mathrm{Da}$ in negative ion mode (Figure 6.4.5) and did give the concomitant fatty acyl chain fragmentation after MS/MS (Figure 6.4.6). Out of the four acids chosen TDPPA, was the 
best candidate to use in comparing the fatty acyl chain fragmentation to more uncomplexed lipids with ammonium acetate in negative ion mode.

Table 6.4.1 Results of diacid-lipid adduct formation in negative ion mode

\begin{tabular}{|c|c|c|c|c|c|}
\hline Entry & Diacid & $\mathrm{pK}_{\mathrm{a}}$ & Solubility*** & $\begin{array}{c}\text { Adduct } \\
\text { formation? }\end{array}$ & $\begin{array}{c}\text { \# of } \\
\text { Atoms } \\
\text { between } \\
\text { charges }\end{array}$ \\
\hline 1 & TBAS & $-3.0,2.0 *$ & soluble & no & 1 \\
\hline 2 & $\mathrm{OA}$ & $1.3,4.0$ & poor & no & 2 \\
\hline 3 & TDPPA & $4.5,5$ & soluble & yes & 7 \\
\hline 4 & RDAA & Undetermined** & very poor & no & 9 \\
\hline
\end{tabular}

- $\mathrm{pKa}$ of sulphuric acid

** no literature on the pKa of this acid

*** Exact concentration of diacid in solution varied depending on its solubility, they were made to be $10 \mathrm{mM}$ if perfectly soluble. 


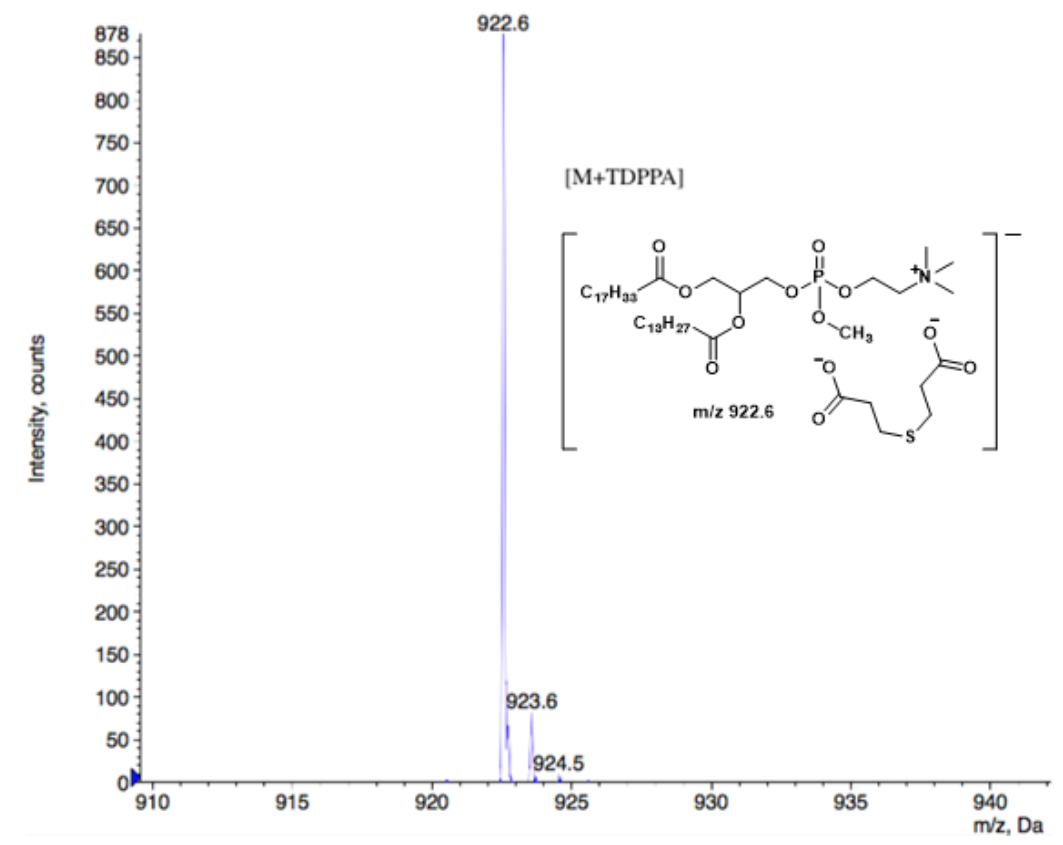

Figure 6.4.5 nESI-MS negative spectrum of TrEnDi derivatized PC with TDPPA

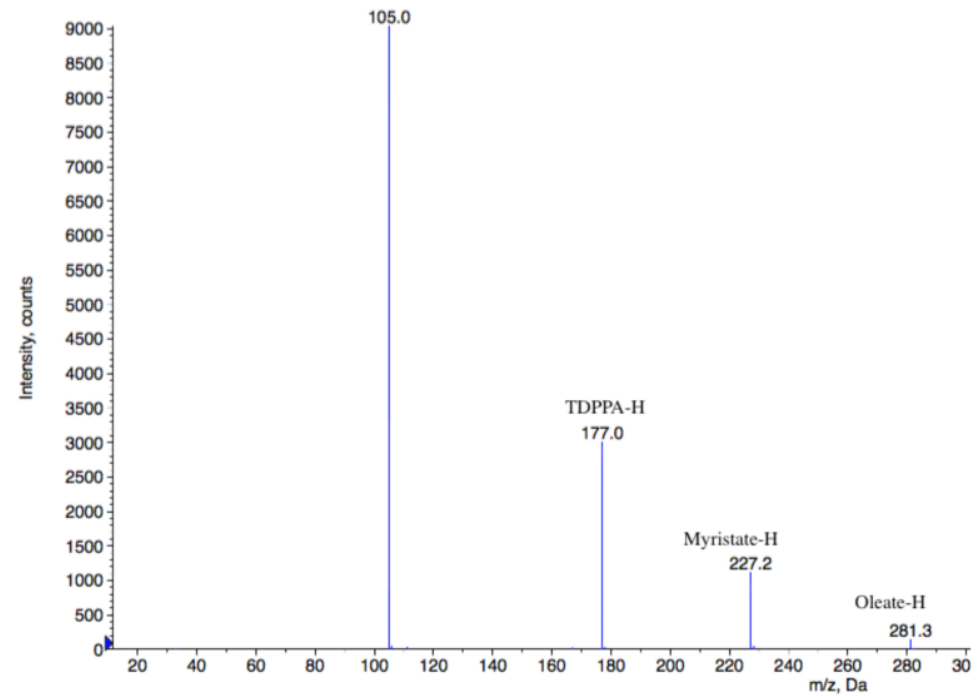

Figure 6.4.6 nESI-MS² negative product ion spectrum of $\mathrm{m} / \mathrm{z} 922.6$

To effectively compare the traditional method of fatty acyl identification to our new method, an external standard was utilized. The first criteria for choosing the standard was that it had to be ionisable in negative ion mode, and secondly was that it must not interfere with adduct formation. Sodium tetraphenylborate was used since it would fulfill both the 
above criteria. The tetraphenylborate has a fixed negative charge as well as borate anions are known to be non-coordinating anions. Figure 6.4.7 shows the ESI-MS of $\mathrm{NaBPh}_{4}$ at 1 $\mu \mathrm{M}$ in ethanol. Interestingly, boron has an unusual isotope pattern, with a significant [M1] peak for ${ }^{10} \mathrm{~B}$, which is $20 \%$ of abundant compared to ${ }^{11} \mathrm{~B}(80 \%)$. A blank with TDPPA and $\mathrm{NaBPh}_{4}$ was performed to identify some of the contaminant peaks found in the comparison experiments.

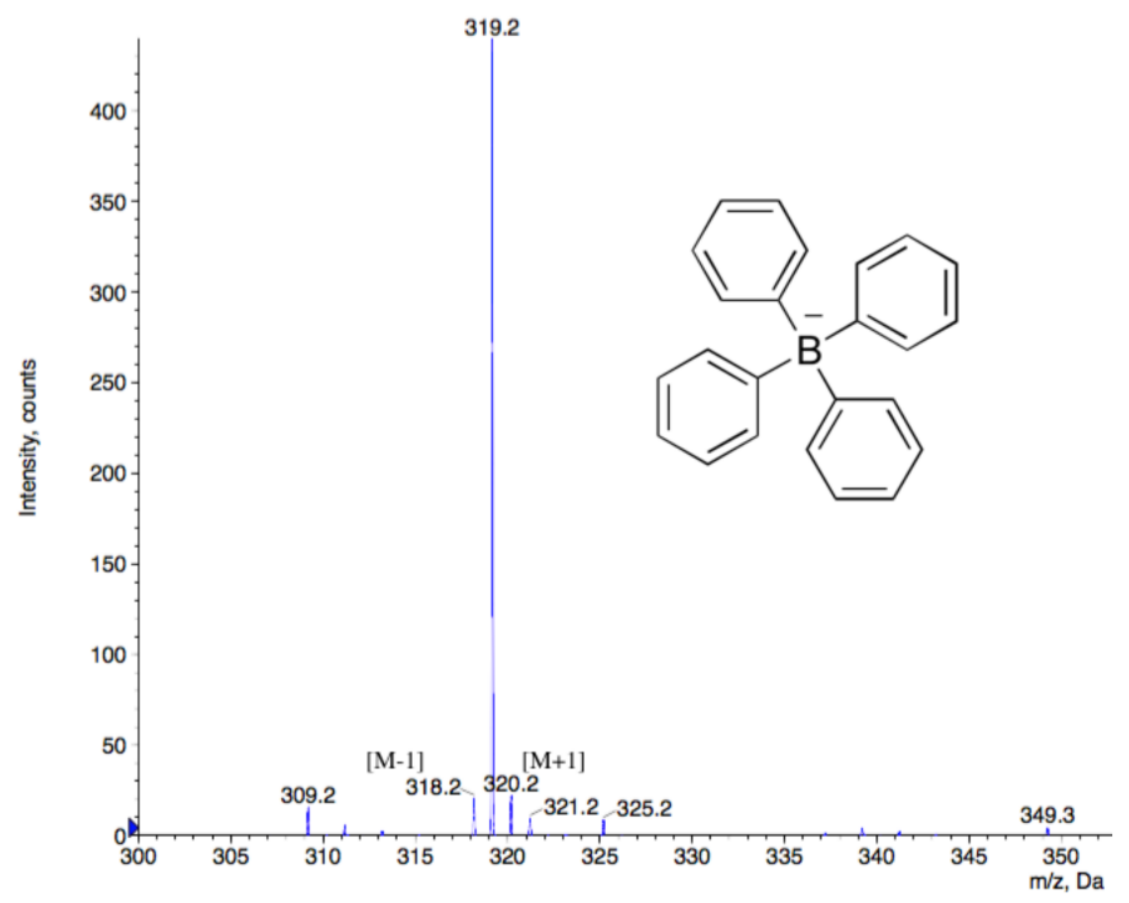

Figure 6.4.7 nESI-MS negative spectrum of $\mathrm{NaBPh}_{4} 1 \mu \mathrm{M}$ in EtOH

With a functional external standard, we set out to compare the methylated lipid in TDPAA (entry 1), non-modified lipid in $\mathrm{NH}_{4} \mathrm{OAc}$ (entry 3), and the non-modified lipid in TDPAA (entry 3). The results are shown in Tables 6.4.2 and 6.4.3. Table 6.4.2 shows all of the peak areas from $\mathrm{NaBPh}_{4}$, peak areas from the formed adduct, and the peak areas from the fatty acids after MS/MS. It is important to note that the parameters such as capillary voltage and source gas pressures were kept the same for both MS and MS/MS scans. This method is not the most rigorous for quantifying the amount of fatty acids being 
cleaved, but due to the lack of time it was acceptable for these proofs of principal experiments.

Table 6.4.3 shows the normalized peak area compared to external standard (analyte peak area/standard peak area area). To our surprise the peak areas of the methylated lipid with TDPPA (entry 1) are approximately an order or magnitude larger than that of nonmodified lipid with $\mathrm{NH}_{4} \mathrm{OAc}$ (entry 2). This means that by using the TrEnDi protocol and adduct formation with TDPPA is more sensitive that the traditional methods of analyzing lipids by MS/MS and could possibly be used to aid in the identification of fatty acyl chains in a biological sample.

Table 6.4.2 Peak areas of comparison experiment

\begin{tabular}{|c|c|c|c|c|}
\hline Entry & Area of BPh4 & Area of Adduct & $\begin{array}{c}\text { Area of FA1 } \\
(227.2 \mathrm{~m} / \mathrm{z})\end{array}$ & $\begin{array}{c}\text { Area of FA2 } \\
(281.3 \mathrm{~m} / \mathrm{z})\end{array}$ \\
\hline 1 & 182.59 & 160.43 & 1.09 & 0.21 \\
\hline 2 & 35300 & 1601.83 & 2.34 & 0.91 \\
\hline 3 & 526.09 & 2121.82 & 20.50 & 5.41 \\
\hline
\end{tabular}

Table 6.4.3 Normalized peak areas of comparison experiment

\begin{tabular}{|c|c|c|c|}
\hline Entry & Area of Adduct & $\begin{array}{c}\text { Area of FA1 } \\
(227.2 \mathrm{~m} / \mathrm{z})\end{array}$ & $\begin{array}{c}\text { Area of FA2 } \\
(281.3 \mathrm{~m} / \mathrm{z})\end{array}$ \\
\hline 1 & 0.879 & 0.00597 & 0.00115 \\
\hline 2 & 0.0453 & $6.63 \times 10^{-5}$ & $2.58 \times 10^{-5}$ \\
\hline 3 & 4.039 & 0.0390 & 0.0103 \\
\hline
\end{tabular}


The most surprizing result was that by using the non-modified lipid with TDPPA was the most sensitive out of the three (entry 3), again having approximately an order of magnitude increase when compared to the methylated variant. What was immediately obvious was that a doubly charged species was observed. This fact was obvious for two reasons, the first being that a mass of 453.7 Da worked out to be exactly half of the expected mass of 907.5 $\mathrm{Da}$, and the second was that the isotope peak spacing was $0.5 \mathrm{Da}$ rather than 1 Da normally observed for singly charged species. Furthermore, the product ion scan of 453.7 Da gave the fatty acid fragmentation as desired proving that the peak was indeed the unmodified PC. This unexpected result reinforces the idea that TDPPA is a good diacid for adduct formation with PC lipids but brings into question if the effectiveness of the TrEnDi protocol. This research has show that it is possible for methylated lipids to undergo charge inversion and fatty acyl cleavage and that further investigation should be performed.

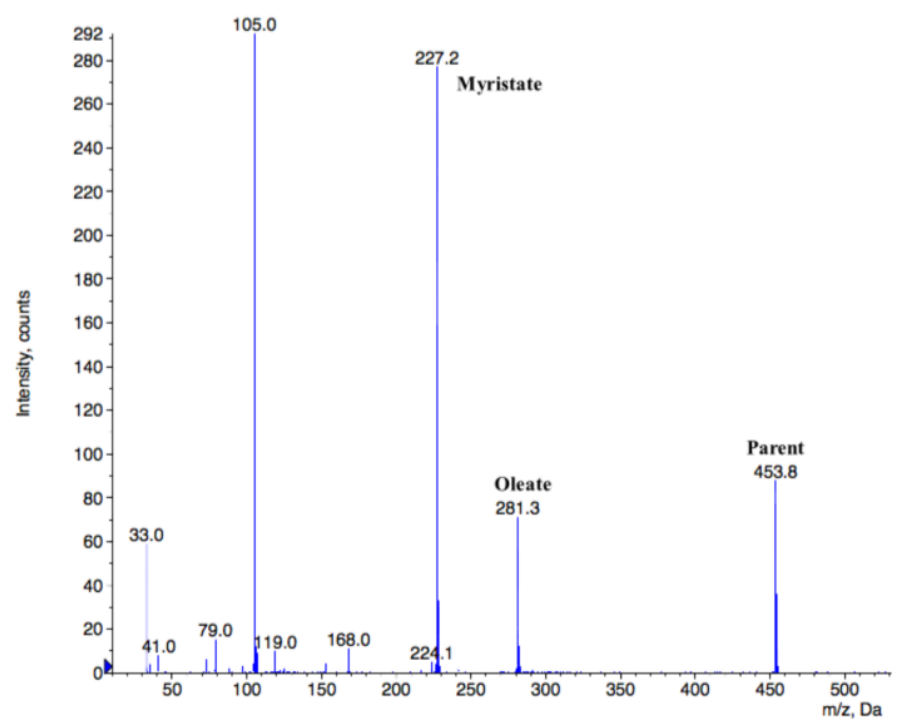

Figure 6.4.8 nESI-MS ${ }^{2}$ negative product ion spectrum of underivatized $[\mathrm{PC}+\mathrm{TDPPA}]^{2-}$ 


\subsection{Conclusion and future works}

We have shown that methylation of a PC is effective at producing the indicative head group fragments in positive ion mode, and no other fragments occur when submitted to MS/MS experiments. Using a novel diacid, TDPPA, the charge inversion of the methylated PC is possible as well as the fragmentation of resultant adduct does yield fatty acyl chains. A new external standard was used to normalize a comparison experiment between modified in TDPPA, non-modified in TDPPA and non-modified in $\mathrm{NH}_{4} \mathrm{OAc}$. The results of this comparison experiment were that the non-modified lipid in TDPPA was the most sensitive, followed by modified in TDPPA and finally non-modified in $\mathrm{NH}_{4} \mathrm{OAc}$. This research was a good example of a proof of concept project, which has a lot of opportunity for optimization.

Some future work to be performed would be to optimize the conditions of adduct formation with methylated $\mathrm{PC}$ as well as testing those conditions with phosphotidylethanolamine. Each of the experiments should be repeated three times to have a statistically significant population size. We should also use a lock mass calibration of our instrument to get high-resolution spectra for further analysis. Finally, optimizing for concentration of the lipid will be important so that we could apply this methodology on a biological sample.

\subsection{References}

(1) Stutzman, J. R.; Blanksby, S. J.; McLuckey, S. A. Anal. Chem. 2013, 85 (7), $3752-3757$. 
(2) Rojas-Betancourt, S.; Stutzman, J. R.; Londry, F. A.; Blanksby, S. J.; McLuckey, S. A. Anal. Chem. 2015, 87 (22), 11255-11262.

(3) de Boer, J.; Backer, H. J. Org. Synth. 2003, No. September, 96-96.

(4) Sigma-Aldrich Co. Technical Bulletin:Diazald $₫$ and Diazomethane Generators https://www.sigmaaldrich.com/content/dam/sigmaaldrich/docs/Aldrich/Bulletin/al_techbull_al180.pdf.

(5) Wasslen, K. V.; Canez, C. R.; Lee, H.; Manthorpe, J. M.; Smith, J. C. Anal. Chem. 2014, 86 (19), 9523-9532.

(6) Sougoule, A. S.; Mei, Z.; Xiao, X.; Balde, C. A.; Samoura, S.; Dolo, A.; Zhu, D. J. Organomet. Chem. 2014, 758, 19-24. 


\section{Chapter 7: Additional Contributions to Studies of Derivatized Glycerophospholipids with Diazoalkanes}

\subsection{Gas-phase Charge inversion of ${ }^{13} \mathrm{C}$-TrEnDi Modified Glycerphospholipids}

\subsubsection{Introduction}

Fragmentation of $[\mathrm{PE}+\mathrm{H}]^{+}$and $[\mathrm{PC}+\mathrm{H}]^{+}$using CID results in ions characteristic of headgroup composition, [PE+H-141] and m/z 184, respectively. However in negative ion mode, PE lipid species are readily deprotonated in source to form [PE-H]', but the quaternary ammonium moiety of $\mathrm{PC}$ lipids requires the formation of $[\mathrm{PC}+\mathrm{X}]^{-}$(where $\mathrm{X}=$ $\mathrm{HCO}_{2}{ }^{-}$or $\mathrm{CH}_{3} \mathrm{O}_{2}{ }^{-}$) to be ionized in negative ion mode. ${ }^{1}$ When these negative ions are subjected to CID fragmentation occurs at $s n 1$ and $s n 2$ to form ions that are characteristic of radyl chain composition. To streamline the process of phospholipid structure elucidation, recent developments in ion trapping mass spectrometers to induce ion-ion reactions have allowed for the characterization of lipid head groups and fatty acyl chain composition using a single ionization polarity. For example, in 2013 McLuckey et al sequentially introduced a doubly deprotonated dicarboxylate $[\mathrm{PDPA}-2 \mathrm{H}]^{2-}$ and protonated lipid $[\mathrm{PC}+\mathrm{H}]^{+}$into a linear ion trap MS (QTrap 4000) to produce a long lived [PC+PDPA$\mathrm{H}]^{-}$net negative product. ${ }^{2}$ Collision induced fragmentation of $[\mathrm{PC}+\mathrm{PDPA}-\mathrm{H}]^{-}$gave $[\mathrm{PC}-$ $\left.\mathrm{CH}_{3}\right]^{-}$which underwent subsequent fragmentation to fatty acyl anions (Scheme 7.1.1). This charge inversion approach facilitated the characterization of PCs and PEs with an increased signal-to-noise ratio compared to standard negative ion mode analysis in complex lipid samples. $^{3}$ 
<smiles>[R]C(=O)OCC(COP(=O)(O)OCC[NH3+])OC([R])=O</smiles><smiles>[R]C(=O)OCC(COP(=O)(O)OCC[N+](C)(C)C)OC([R])=O</smiles>

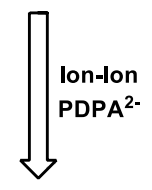<smiles>[R]C(=O)OCC(COP(=O)([O-])OCCN)OC([R])=O</smiles><smiles>[R]C(=O)OCC(COP(=O)(O)OCC[N+](C)(C)C)OC([R])C(=O)O</smiles>
CID<smiles>[R]C(=O)[O-]</smiles>

Diagnostic ions produced from [PE-H]<smiles>[R]C(=O)OCC(COP(=O)([O-])OCCN(C)C)OC([R4])=O</smiles><smiles>[R]C(=O)[O-]</smiles>

Diagnostic ions produced from [PC+PDPA]

Scheme 7.1.1 Gas-phase charge inversion with PDPA and fragmentation of PC and PE lipids

\subsubsection{Trimethylation Enhancement Using ${ }^{13}$ C-DZM: Gas-Phase Charge Inversion of Modified Phospholipid Cations for Enhanced Structural Characterization}

Figures are reproduced with permission from: Betancourt, S.K.; Canez, C.R.; Shields, S. W.; Manthorpe, J. M.; Smith, J. C.; McLuckey, S. A. Anal. Chem. 2017, 89, 17, 94529458 
The major limitation of ${ }^{13} \mathrm{C}$-TrEnDi modified phospholipid (tmPL) species is the inability to characterize fatty acyl components via classical tandem mass spectrometry techniques. This work reported here was motivated by the benefits provided by signal enhancement from ${ }^{13} \mathrm{C}$-TrEnDi and gas-phase charge inversion for a more complete structural characterization of fatty acyl components. Ion trap CID in positive ion mode of $[\mathrm{tmPC}]^{+},[\mathrm{tmPE}]^{+}$and $[\mathrm{tmPS}]^{+}$provides headgroup cations at $\mathrm{m} / \mathrm{z}$ 199, 202, and 260 respectively as the dominant fragment. Ionization in negative ion mode with common solvent additives like acetate cannot be used since the adduction with quaternary ammonium site neutralizes the tmPL and a negative ion will not be produced.

However, when tmPLs are subjected to gas-phase charge inversion with PDPA this generates a fragile complex that readily fragments into the ions summarized in Figure 7.1.1. Initially, the abstraction of a methyl group was by PDPA during the ion-ion reaction was observed for all tmPL. The dominant process after methyl abstraction is the transfer of labelled choline (tmPC and tmPE) or serine (tmPS) to PDPA dianion after residence in the ion trap to form a neutral diester of PDPA and negatively charged ${ }^{13} \mathrm{C}$-phosphatidic acid (Figure 7.1.2). A secondary process was observed for tmPC and tmPE where a second methyl abstraction was observed rather than headgroup transfer. Although PC and PE standards have the same fatty acyl chains, their differentiation can be accomplished by ion trap CID tmPL+PDPA complexes. The activation of [tmPC+PDPA-2H] gives the product $\left[\mathrm{tmPC}-{ }^{13} \mathrm{CH}_{3}-\mathrm{CH}_{3}\right]^{-}$whereas activation of $[\mathrm{tmPE}+\mathrm{PDPA}-2 \mathrm{H}]^{-}$gives the product $[\mathrm{tmPC}-$ $\left.{ }^{213} \mathrm{CH}_{3}\right]^{-}$. Similarly, lipid class information can be determined from neutral losses from the formed [tmPL+PDPA-2H] ${ }^{-}$during the ion/ion reaction. For example, neutral losses of 307, 310 and $369 \mathrm{Da}$ are indicative of tmPC, tmPE and tmPS lipid classes, respectively. 

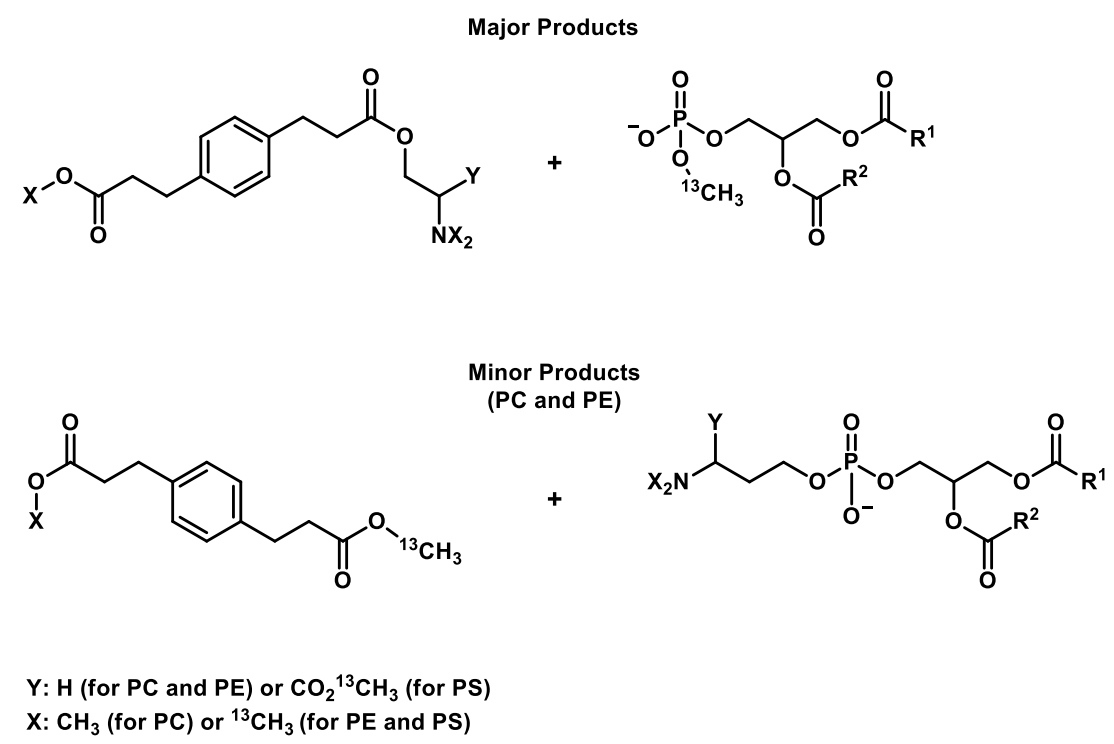

Figure 7.1.1 Ion-ion reaction products of tmPE tmPC and tmPS standards with PDPA 


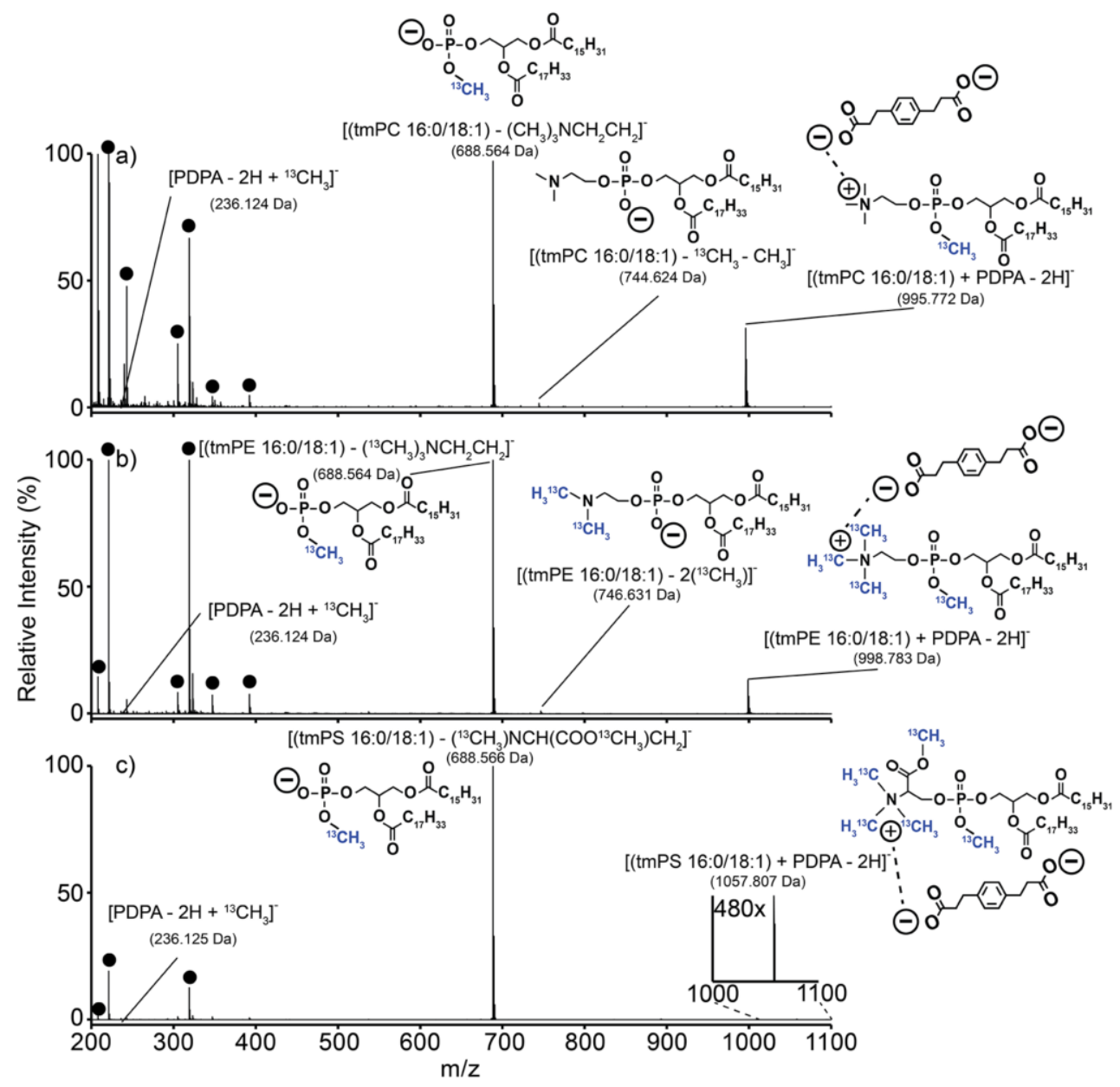

Figure 7.1.2 Ion-ion reaction between reagent dianion [PDPA-2H] $]^{-}$and (a) tmPC,

(b) tmPE and (c) tmPS. (•) Ions are present prior to cation injection

The most abundant product of the ion/ion reaction could then be subjected to iontrap CID to investigate their fatty acyl chain composition. Figure 7.1.3a shows the product ions observed from the activation of $\left[\mathrm{tmPC}-\left(\mathrm{CH}_{3}\right)_{3} \mathrm{NCH}_{2} \mathrm{CH}_{2}\right]^{-}$. The spectrum contains abundant fatty acyl product anions (carboxylate) at m/z 255.26 ([C16:0-H] $\left.]^{-}\right)$and 281.27 ([C18:1-H]). The abundance ratio between fatty acid carboxylated anions confirms their glycerol chain position as 16:0/18:1 $(s n 1 / s n 2)$ due to $\mathrm{m} / \mathrm{z} 281.27$ having the highest abundance. Similar fragmentation profile of $\left[\mathrm{tmPE}-\left(\mathrm{CH}_{3}\right)_{3} \mathrm{NCH}_{2} \mathrm{CH}_{2}\right]^{-}$and [tmPS- 
$\left.\left(\mathrm{CH}_{3}\right)_{3} \mathrm{NCH}_{2} \mathrm{CH}_{2}\right]^{-}$are shown in Figure 7.1.3b,c. Through charge inversion of tmPLs with PDPA followed by CID activation of major gas-phase reaction products, lipid headgroup identification as well as fatty acyl composition can be determined.

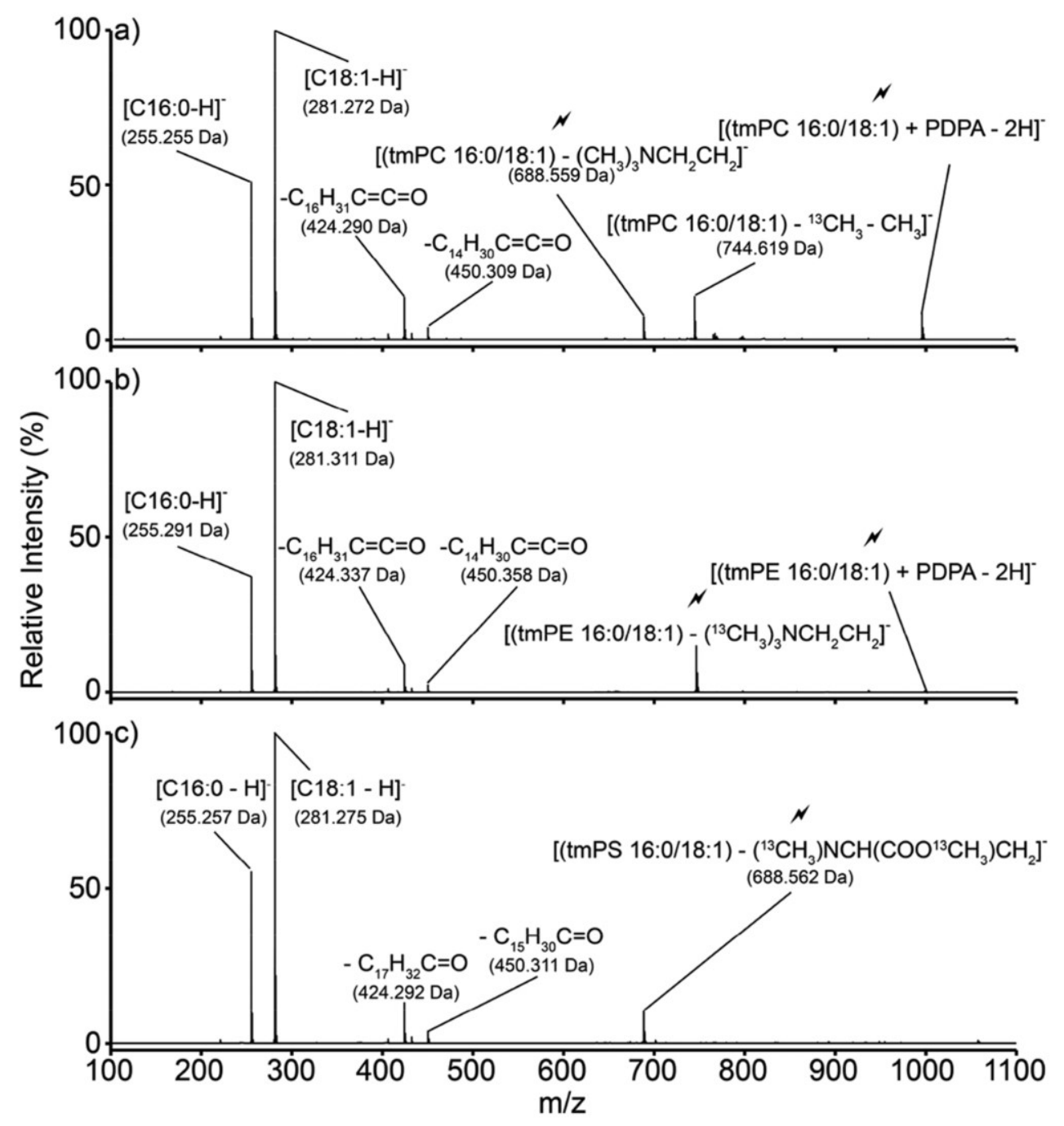

Figure 7.1.3 Ion-trap CID of (a) [tmPC- $\left.\left(\mathrm{CH}_{3}\right)_{3} \mathrm{NCH}_{2} \mathrm{CH}_{2}\right]^{-}$, (b) [tmPE$\left.\left(\mathrm{CH}_{3}\right)_{3} \mathrm{NCH}_{2} \mathrm{CH}_{2}\right]^{-}$, [tmPS- $\left.\left(\mathrm{CH}_{3}\right)_{3} \mathrm{NCH}_{2} \mathrm{CH}_{2}\right]^{-}$. Lightning bolt signifies activated ions. 


\subsubsection{Determination of Double bond location in ${ }^{13} \mathrm{C}$-TrEnDi modified lipids using PDPA charge inversion and Paternó-Büchi Reaction}

**This work was performed in collaboration with Scott McLuckey (PI) and Elissia Franklin (PhD student) from Purdue University, West Lafayette IN, USA

The photochemical [2+2] cycloaddition of aldehydes and alkenes to yield an oxetane product has been known since the early 1900s. ${ }^{4}$ Briefly, the Paternó-Büchi (PB) reaction consists of carbonyl containing compounds which are activated by UV radiation to form a high-energy electronic state that interacts with alkene $\pi$ system. Recently, the development of an in-line flow reactor using acetone as a co-solvent and $254 \mathrm{~nm}$ UV light, has been used to cyclize the unsaturations of fatty acid before ESI-MS ${ }^{2}$ analysis to definitively locate the alkene position. ${ }^{5}$ The PB reacted fatty acids after ionization and subsequent low energy CID were observed to produce diagnostic product ions having neutral losses corresponding to fragmentation at the oxetane ring (Scheme 7.1.2). The mass difference between diagnostic product ions and the parent ion the location of alkene can be inferred. Xia et al in 2019 applied this methodology together with gas-phase charge inversion to enhance de novo structure elucidation of GPLs. ${ }^{6}$ To increase the accuracy of low abundant GPL identification, ${ }^{13} \mathrm{C}-\mathrm{TrEnDi}$ derivatization could be added to the workflow described above. Scheme 7.1.2 shows the process by which tmPLs undergo complete structural elucidation. The process is similar to what was described in Section 7.1.2 with the addition of the PB reaction on tmPLs and an additional MS event to fragment fatty acyl components to generate product ions that describe the location of double bonds. 


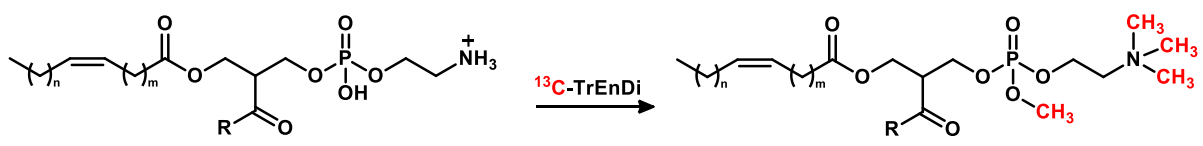

$[\mathrm{GP}+\mathrm{H}]^{+}$

$[\mathrm{tmGP}]^{+}$

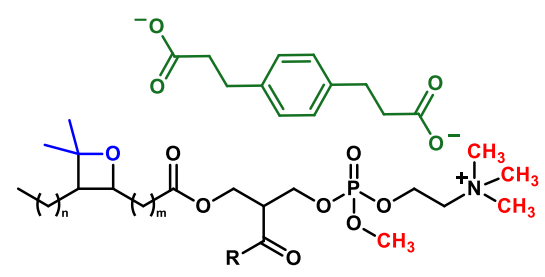

[ ${ }^{\mathrm{PB}}$ tm GP+PDPA-2H] $^{-}$

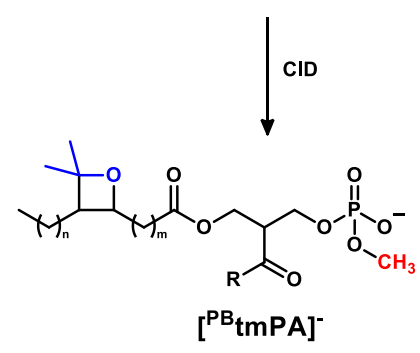

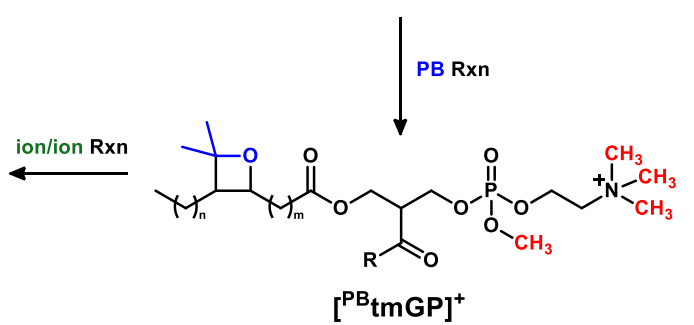

[PB $\left.^{\mathrm{PmGP}}\right]^{+}$
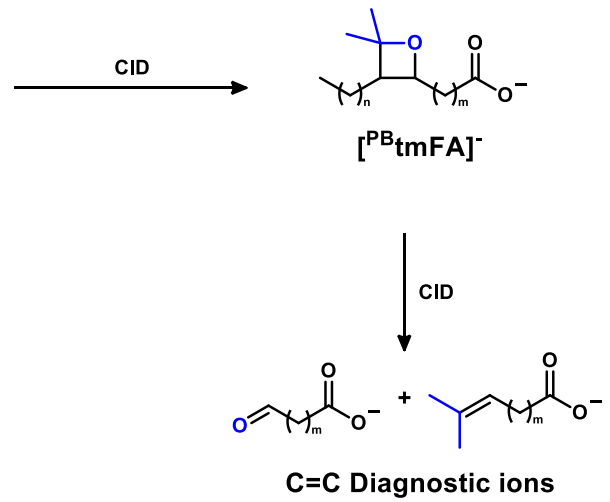

Scheme 7.1.2 Sequence of events for tmPL structure determination using off-line Paternó-Büchi $[2+2]$ cycloaddition and ion/ion reaction products analized via $\mathrm{MS}^{4}$

The initial derivatization of tmPE with acetone and $253 \mathrm{~nm}$ light yielded 15 of the ${ }^{\mathrm{PB}}$ tmPE product (Figure 7.1.4a) The tmPE species appeared at $\mathrm{m} / \mathrm{z} 778$ and $\mathrm{PB}$ reaction product gave ions with a neutral gain of $58 \mathrm{Da}(\mathrm{m} / \mathrm{z} 863)$. This $\left[{ }^{\mathrm{PB}} \mathrm{tmPE}\right]^{+}$was introduced into the mass spectrometer via direct nESI and stored mutually with $[\mathrm{PDPA}-2 \mathrm{H}]^{2-}$. As expected, after the ion-reaction a charge inverted complex was formed $\left[{ }^{\mathrm{PB}} \mathrm{tmPE}+\mathrm{PDPA}-\right.$ $2 \mathrm{H}^{-}$at $\mathrm{m} / \mathrm{z} 1056$ and anionic ${ }^{13} \mathrm{C}$-methylated phosphatidic acid $\left(\left[{ }^{\mathrm{PB}} \mathrm{tmPA}\right]^{-}\right)$at $\mathrm{m} / \mathrm{z} 746$ (Figure 7.1.4b). This result suggests that addition of an oxetane ring into the fatty acyl 
moiety does not significantly affect the outcome of ion/ion reaction. After activation via CID $\left[{ }^{\mathrm{PB}} \text { tmPA }\right]^{-}$readily produced fatty acyl components $[\mathrm{C} 16: 0-\mathrm{H}]^{-}$and $\left[{ }^{\mathrm{PB}} \mathrm{C} 18: 1-\mathrm{H}\right]^{-} \mathrm{m} / \mathrm{z}$ 255 and 339, respectively. After isolation and CID $\left(\mathrm{MS}^{4}\right) \mathrm{m} / \mathrm{z} 339$ provided diagnostic FA product ions m/z 197 as a dimethyl alkene and 171 as aldehyde. From the neutral loss of 142 and 168 Da respectively from $\left[{ }^{\mathrm{PB}} \mathrm{C} 18: 0-\mathrm{H}\right]^{-}$the location of carbon-carbon double bond can be determined to be $\Delta^{9}$ in the derivatized PE (Figure 7.1.4d).
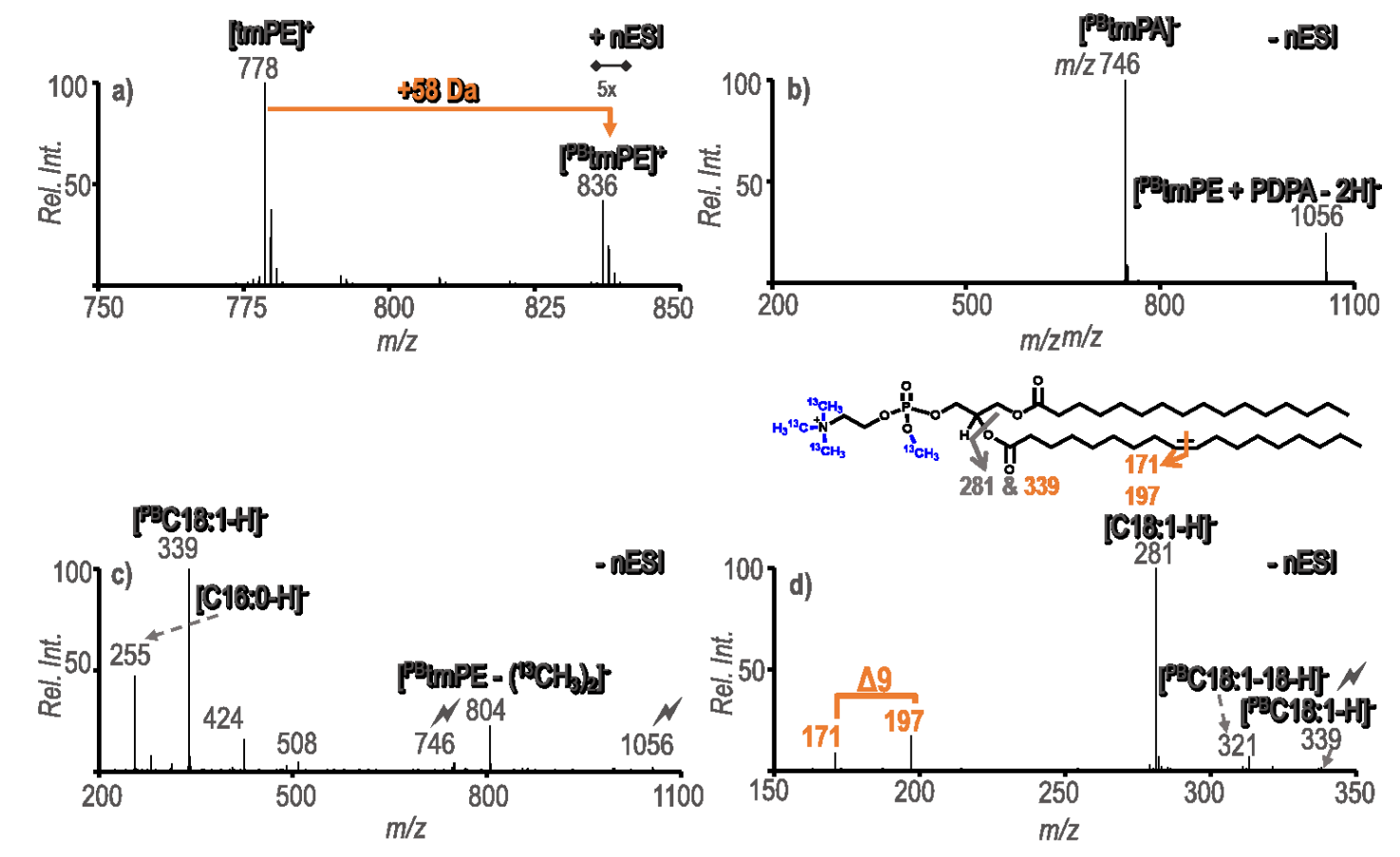

Figure 7.1.4 Combination of $P B$ reaction and charge inversion of tmPE. a) The PB reaction spectrum of $5 \mu M \mathrm{tm} P E$ 16:0/18:1(9Z) after 5 seconds of $U V$ exposure, b) Gas-phase ion/ion reaction of $\left[{ }^{\mathrm{PB}} \mathrm{tmPE}\right]^{+}$and $[\mathrm{PDPA}-2 \mathrm{H}]^{2-}$, c) Ion trap CID of $\left.{ }^{{ }^{\mathrm{PB}} \mathrm{tmPE}}+\mathrm{PDPA}-2 \mathrm{H}\right]^{-}$followed by ion trap CID of $\left[{ }^{\mathrm{PB}} \mathrm{tmPA}\right]^{-}$, d) Ion trap CID of

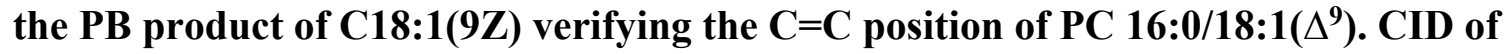
a target ion is depicted by a lightning bolt $(\mathcal{N})$. 
To investigate the usefulness of our methodology on a complex lipid extract we opted to work with commercial bovine liver extract (BLE). In previous analysis of BLE, PE 36:3 could not be fullly characterized using this methodology due to its very low abundance. However, after ${ }^{13} \mathrm{C}$-TrEnDi modification fatty acyl chain composition and double bond location could be analyzed. Three distinct acyl chain compositions that were observed for $\mathrm{PE}(36: 3)$ were 18:0/18:3 (major), 18:1/18:2 and 16:0/20:3 (minor). Figure 7.1.5a) shows the CID spectrum produced by the activation of $\left[{ }^{\mathrm{PB}} \mathrm{C} 18: 1\right]^{-}$that originated from $\operatorname{tmPE}(36: 3)$. Two sets of C-C double bond diagnostic fragments were found to be present indicating $\Delta^{9}$ and $\Delta^{11}$ monounsaturated $\mathrm{C} 18$ fatty acid isomers. Figure 7.1.5b shows the CID spectrum produced by the activation of $\left[{ }^{\mathrm{PB}} \mathrm{C} 18: 2\right]^{-}$and each double bond location are assigned as $\Delta^{9}$ and $\Delta^{12}$. Therefore, within $\operatorname{PE}(18: 1 / 18: 2)$ two structural isomers can unequivocally be assigned as $\operatorname{PE}\left(18: 1, \Delta^{9} / 18: 2, \Delta^{9}, \Delta^{12}\right)$ and $\operatorname{PE}\left(18: 1, \Delta^{11} / 18: 2, \Delta^{9}, \Delta^{12}\right)$. These results exemplify the structural diversity of the lipodome, particularly regarding the presence of GPL species with two fatty acyl chains and increased sensitivity due to ${ }^{13} \mathrm{C}$ TrEnDi modification can be beneficial for characterizing absolute lipid structure. 


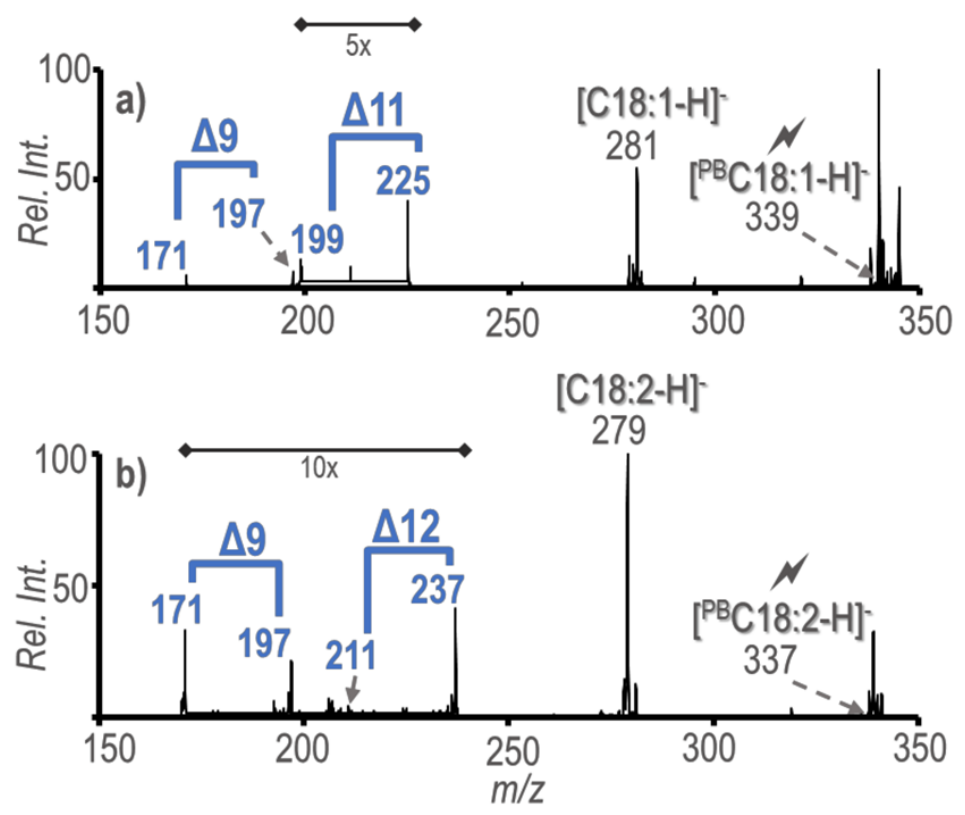

Figure 7.1.5 a) Charge inversion of positive ion mode $\mathrm{m} / \mathrm{z} 860$ (the PB product of 802), and sequential CID spectrum of $m / z, 339$ (a) and sequential CID spectrum of $m / z 337$ (b). CID of a target ion is depicted by a lightning bolt $(\mathcal{N})$

\subsection{Ultraviolet photodissociation of ${ }^{13} \mathrm{C}$-TrEnDi modified glycerophospholipids}

\subsubsection{Introduction}

A crucial characteristic of any analysis of biomolecules via mass spectrometry is the ability to dissociate ions in the gas phase. There exist many different methods of dissociation that can relay different chemical and physical properties of the analyte in question. Some of the most common techniques include, the collision of accelerated ions with a unreactive gas (collision induced dissociation) ${ }^{7}$, the transfer of an electron to ions to form radical cations (electron transfer dissociation $)^{8}$ and the absorption of one or more photons by ions to produce high energy electronic and vibrational states (photodissociation). ${ }^{9}$ The dissociation of ions and analysis via mass spectrometry, referred 
to as tandem mass spectrometry $\left(\mathrm{MS}^{2}\right)$, provides the analytical chemist with the ability to structurally characterize biomolecules in extremely low quantities ( $\mathrm{nmol}-\mathrm{fmol})$.

Ultraviolet photodissociation (UVPD) is a process by which ions are irradiated with a UV laser, absorb one or multiple photons then dissociate to produces unique diagnostic fragment ions. In comparison, UV absorption of gas-phase ions allows access to highly energetic vibronic states above the bond dissociation threshold that traditional collisionally activated ions cannot achieve. ${ }^{10}$ Furthermore, the amount of energy added to ions can be directly controlled by the wavelength of laser light used, which can increase the control over the desired dissociation pathways are activated. An example is the absorption of the indole ring of a small protonated tryptophan-containing peptide using $263 \mathrm{~nm}$ photons to give a radical cation to produce fragments (Scheme 7.2.1). ${ }^{11}$ This type of ion activation and fragmentation has applications in the identification of peptides, oligonucleotides, carbohydrates and lipids to improve their identification. ${ }^{12}$

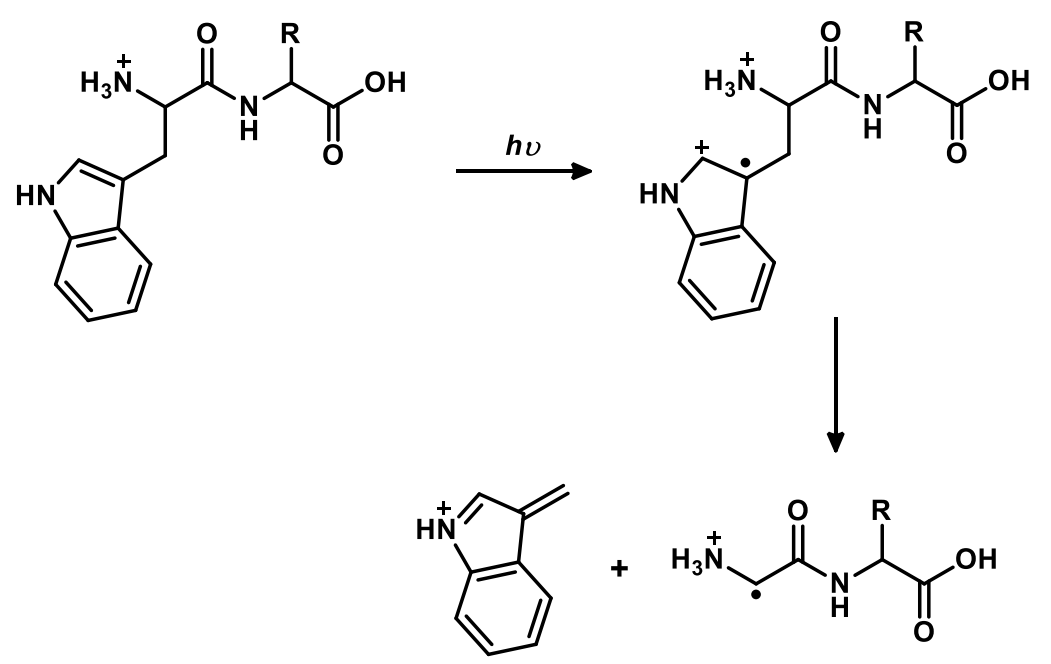

Scheme 7.2.1 UVPD Ion Activation with 263 nm Laser to Cause Fragmentation 
The advancement ion manipulation techniques, such as ion traps, have readily enabled UVPD as a practical method of ion activations. Ion traps use direct current (DC) and alternating currents (AC) to trap gas-phase ions in a potential energy well, in effect ions can be packaged and trapped in a confined space that can be efficiently irradiated with photons. ${ }^{13}$ The modification of an orbitrap mass spectrometer to implement UVPD in 2011, involved Brodbelt et al. installed a window into the higher energy collision (HCD) cell where a laser could be mounted to irradiate the ions within. ${ }^{14}$ Figure 7.2.1 is a representation of the first commercially available ion trap mass spectrometer with UVPD capabilities. The schematic shows an ion trap where ions can be accumulated and either collisionally activated (HCD) or photo activated (UVPD) to induce dissociation and subsequent high-resolution mass spectrometric (HRMS) analysis.

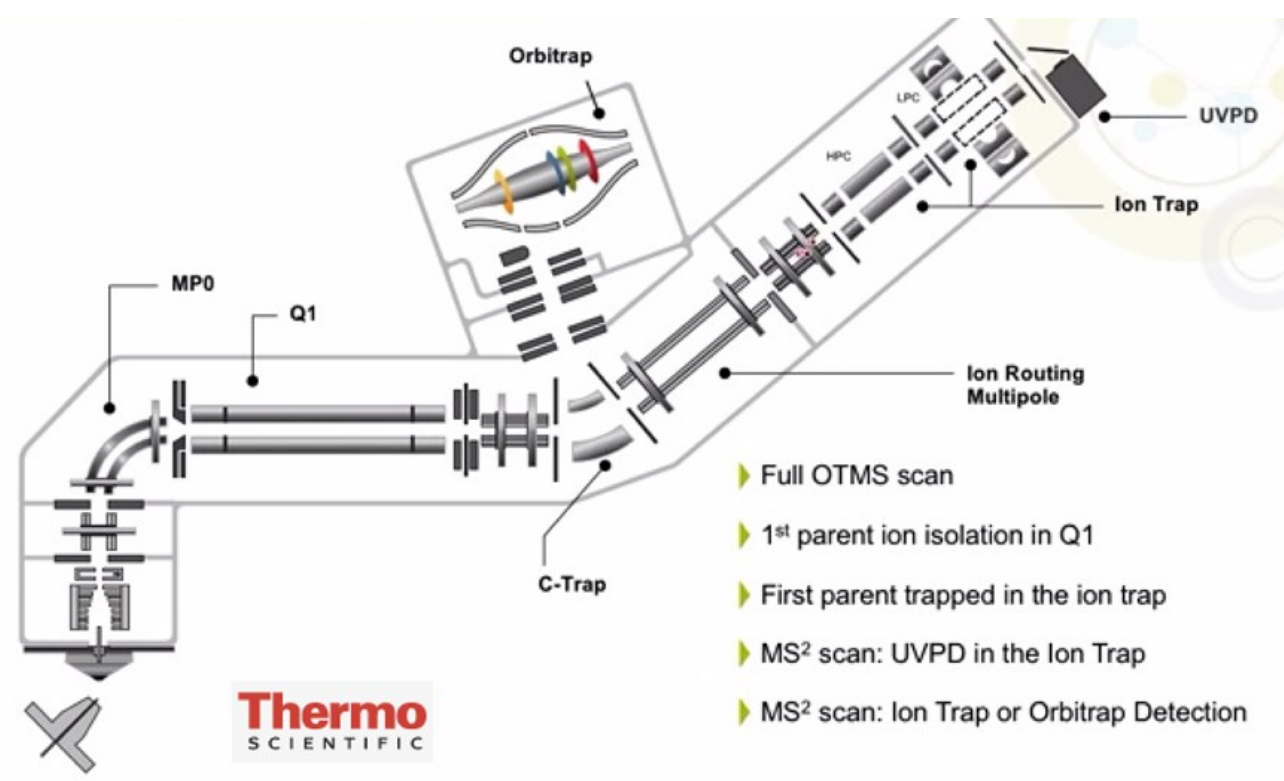

Figure 7.2.1 Schematic of the Orbitrap Fusion Lumos tribrid mass spectrometer from Thermo Scientific ${ }^{15}$ 
As mentioned in Chapter 1 of this thesis, the absolute structural characterization of lipid structures is crucial to comprehending their biological activity in many complex systems. Lipids are extremely structurally diverse biomolecules and thus require the use of tandem mass spectrometry to correctly characterize lipids species. Over the last few years UVPD, as a method of ion activation has emerged to facilitate a deeper investigation into the structural characteristics of sphingolipids, glycolipids, cardiolipins and cyclopropane glycerophospholipids. In this section the diagnostic fragmentation of three different lipid classes using UVPD is briefly reviewed.

Diphosphatidyltrehalose (diPT, Figure 7.2.2a) is a glycolipid recently identified in a typhoid fever-causing bacterium Salmonella typhi that was shown to act as a immunostimulant. ${ }^{16}$ This glycolipid can exist as two different regioisomers where the acyl chain positions are reversed at sn1 and sn2. The two regioisomers were synthesized as reference materials and subjected to nuclear magnetic resonance spectroscopy and tandem mass spectrometry to identify the regioisomers. ${ }^{17} \mathrm{MS}^{3}$ using a hybrid CID and UVPD approach was able to determine the absolute position of each acyl chain. Figure 7.2.2B shows a key fragment from $[\mathrm{diPT}+\mathrm{Na}]^{+}$after $\mathrm{HCD}$ and UVPD sequential activation to unambiguously assign fatty acyl chain $16: 0$ to $\operatorname{sn} 1$ and $16: 0$ ( $\Delta^{9}$ cyclopropane) to $s n 2$ in the natural product.

Cyclopropane fatty acids like mycolic acid (MA) are commonly found in bacterial lipidomes and the location of the cyclopropane in the fatty acyl group is known to affect biological activity. ${ }^{18}$ Recently, Brodbelt et al were able to use UVPD to definitively determine cyclopropane location at C16 and C32 in MA. Mycolic acid, when activated with UV radiation readily fragments at the cyclopropane in a hydrocarbon chain to give 
diagnostic fragments that appear with a difference of $14 \mathrm{Da}$ and the exact position can be calculated from the mass of the neutral fragment lost.

Similarly, the position of an alkene in unsaturated fatty acyl chains of cardiolipins can be determined via HCD followed by UVPD activation. ${ }^{19}$ Cardiolipins are a unique class of lipid molecules that consist of two phosphatidic acid moieties connected 1,3 by a central glycerol resulting in a total of four acyl chains. Double bond localization is a challenging aspect of the complete structural characterization of CLs using classical tandem mass spectrometry techniques. A hybrid activation technique was utilized for CL characterization fatty acid and phosphatidic acid composition could reliably be achieved via CID and double bond location could readily be determined via UVPD. Location of the double bonds in CL standard could be accomplished by the production of a pair of peaks with a difference of $24 \mathrm{Da}$ at $\mathrm{m} / \mathrm{z} 1059.63$ and 1035.63. Once again, through calculation of neutral loss from each of these fragments, the position was readily determined to be $\Delta^{9}$. These examples serve to demonstrate that ultraviolet photodissociation is useful as a method of breaking $\mathrm{C}-\mathrm{C}$ and $\mathrm{C}=\mathrm{C}$ bonds in tandem mass spectrometry for in-depth de novo structure determination of many lipid classes. 

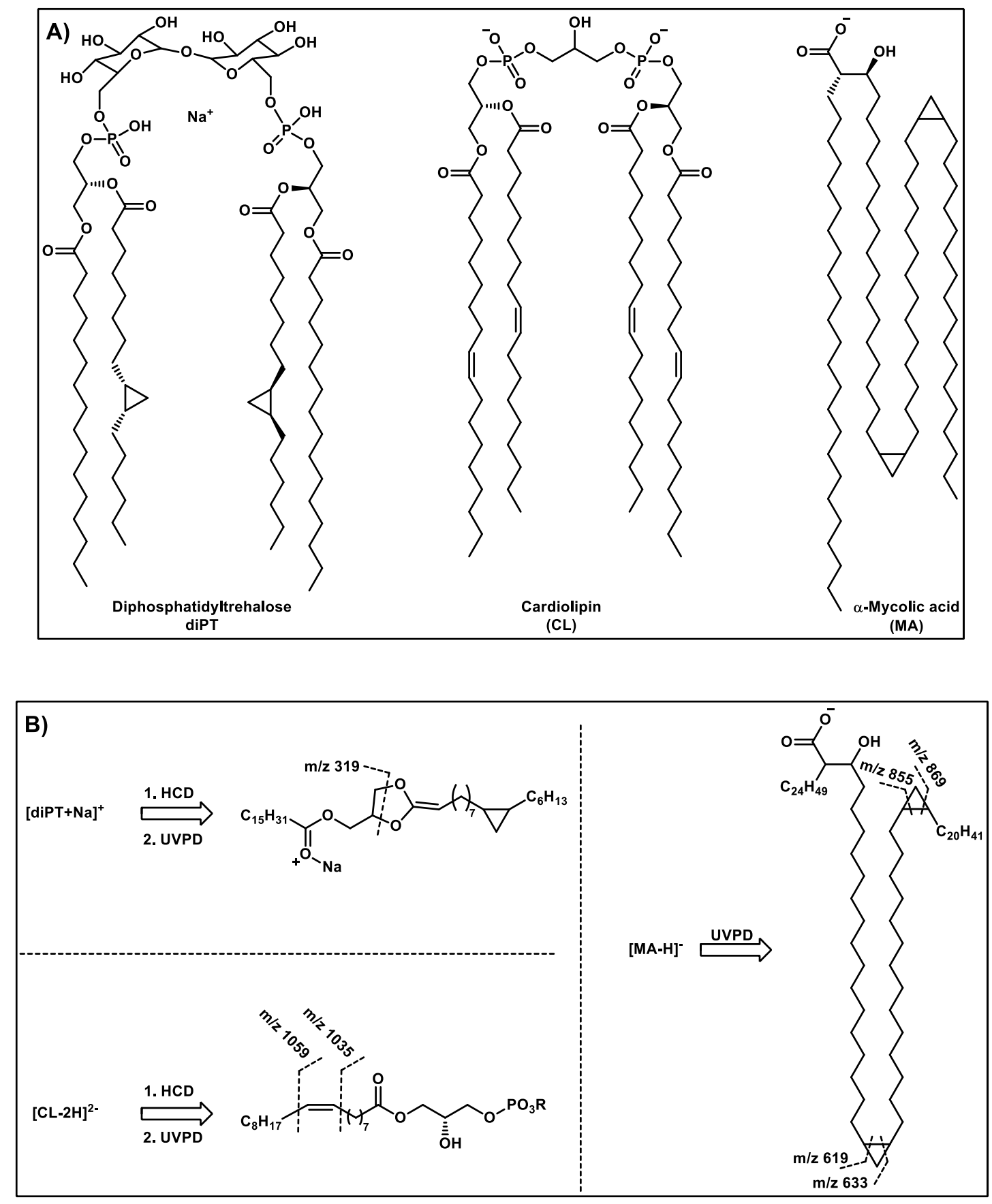

Figure 7.2.2 Chemical structures of diphosphatidyltrehalose, cardiolipin (18:1/18:1/18:1/18:1) and mycolic acid (A). Diagnostic fragments produced via a hybrid CID-UVPD MS ${ }^{2}$ analysis of $[\mathrm{diPT}+\mathrm{Na}]^{+},[\mathrm{CL}-2 \mathrm{H}]^{-}$, and $[\mathrm{MA}-\mathrm{H}]^{-}$enabling a complete structure elucidation (B) 


\subsubsection{UVPD of TrEnDi Modified Glycerophospholipids}

**This work was performed in collaboration with Jennifer Brodbelt (PI) and Molly Blevins (PhD student) from University of Texas at Austin, Austin TX, USA.

Diagnostic fragment ions formed from the CID activation of ${ }^{13} \mathrm{C}-\mathrm{TrEnDi}$ modified phospholipids is limited to the determination of headgroup composition. Therefore, additional activation techniques must be used to streamline analyses that attempt to characterize low abundance lipid species. This section shows the benefits of increasing ion abundance via the quaternization of PE lipid classes in combination with UVPD fragmentation to determine double bond position. Initially, PE $\left(16: 0 / 18: 1, \Delta^{9}\right)$ was exposed to ${ }^{13} \mathrm{C}$-DZM in solution to generate $\left.[\mathrm{tmPE}]\right]^{+}$and analyzed via direct $\mathrm{nESI}$ on a UVPD capable instrument. The product spectrum produced by HCD activation of methylated PE is observed in Figure 7.2.3(top). As expected, the most abundant product ion was $\mathrm{m} / \mathrm{z}$ 202.10 which is consistent with quadruply ${ }^{13} \mathrm{C}$ methylated PE headgroup as well as a minor fragment consistent with $\left[\mathrm{tmPE}-{ }^{13} \mathrm{CH}_{3} \mathrm{PO}_{4} \mathrm{C}_{2} \mathrm{H}_{4} \mathrm{~N}\left({ }^{13} \mathrm{CH}_{3}\right)_{3}\right]^{+}$. However, when subjected to UVPD activation with 10 pulses $(6 \mathrm{~mJ})$ of a $213 \mathrm{~nm}$ laser, fragments consistent with double bond location can be observed. Due to the activation with UV light, rather than collisional activation higher energy fragmentation pathways are revealed (Figure 7.2.3(bottom)). Diagnostic fragment ions $\mathrm{m} / \mathrm{z} 664.48$ and 640.48 are readily identifiable as the cleavage fatty acyl alkene by their 24 Da mass difference. These ions represent neutral losses of $138.14\left(\mathrm{C}_{10} \mathrm{H}_{18}\right)$ and $114.14\left(\mathrm{C}_{8} \mathrm{H}_{18}\right) \mathrm{Da}$ and double bond position can be labeled as $\Delta^{9}$ (or $\left.\omega^{9}\right)$. 


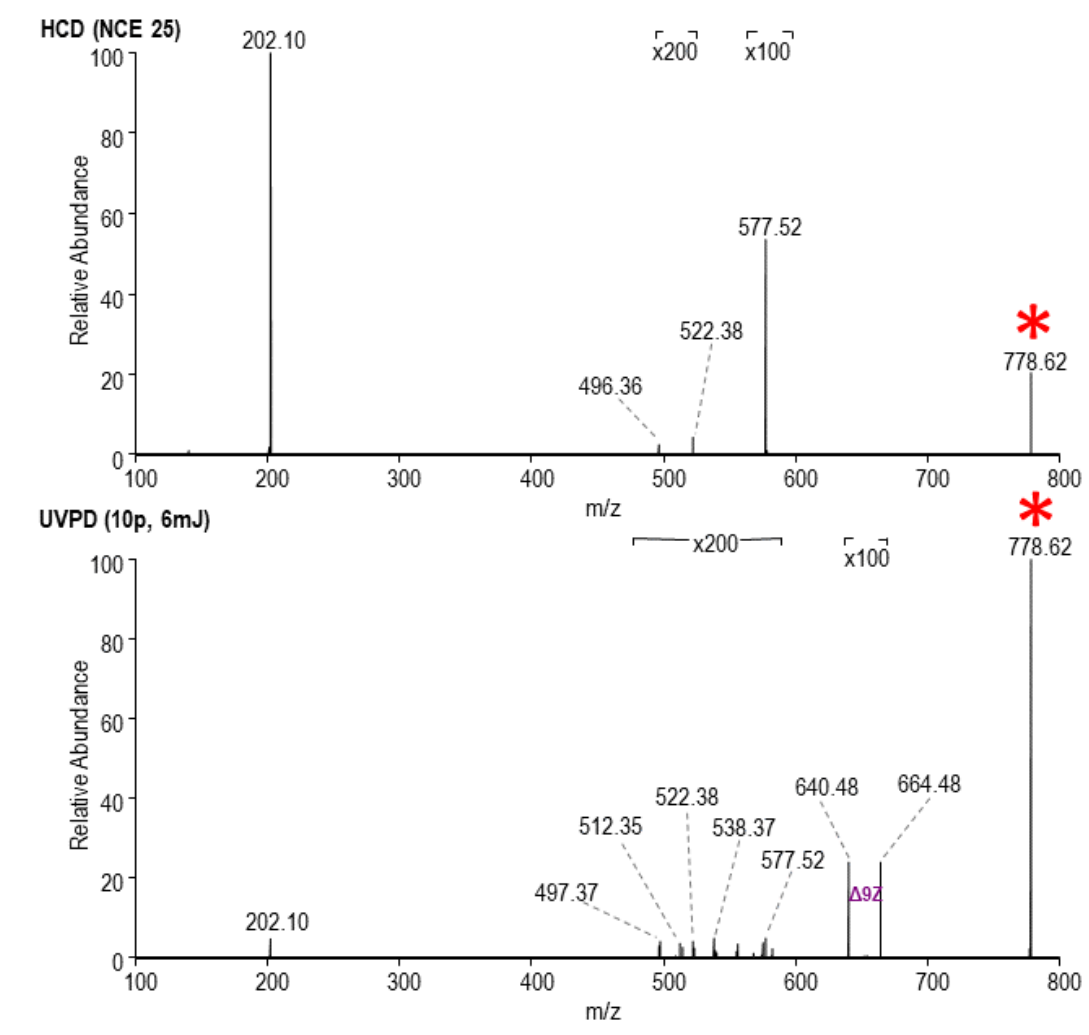

\section{Figure 7.2.3 HCD (top) and UVPD (bottom) product ion spectra of $[\operatorname{tmPE}(16: 0 / 18: 1)]^{+}$}

Mass to charge peak areas of diagnostic ions generated via UVPD fragmentation of ${ }^{13} \mathrm{C}$-tmPE(16:0/18:1) and unmodified PE standard using nESI (positive and negative mode) were plotted as a function of the number of moles injected (Figure 7.2.4). The limit of detection (LOD) and limit of quantitation (LOQ) was defined as having a m/z peak area with a signal-to-noise ratio of three and ten, respectively. Sensitivity increase of tmPE diagnostic peaks, based on LOD measurements were observed to be 3.5-fold compared to negative nESI and 4.0 -fold for positive nESI. The enhancements provided by ${ }^{13} \mathrm{C}-\mathrm{TrEnDi}$ modification show promising increase in abundance of $\mathrm{C}=\mathrm{C}$ diagnostic ion for a more accurate structural characterization. 


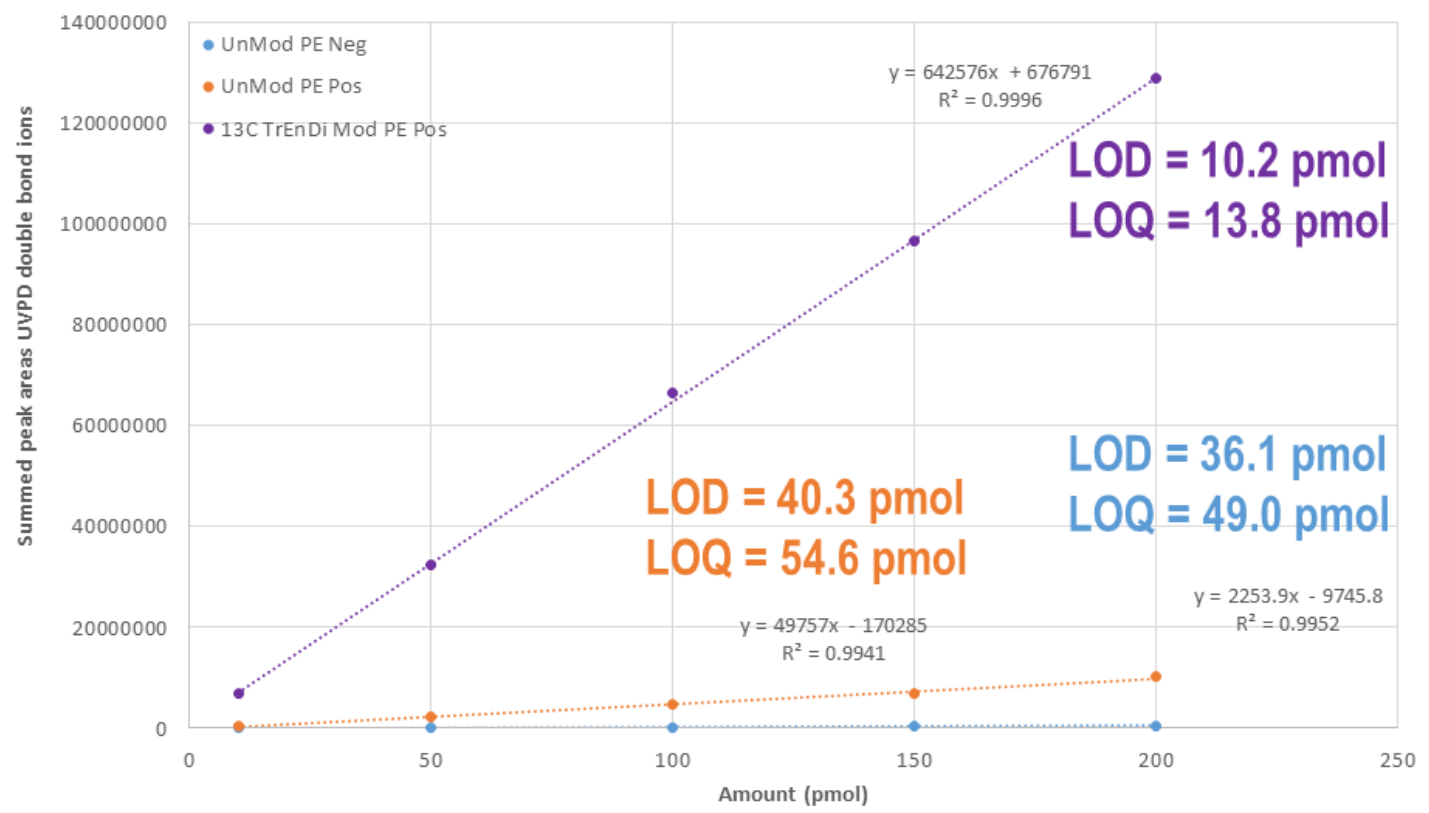

Figure 7.2.4 Summed UVPD DB frag ion peak area vs. \#mol injected

Our ultimate goal is to implement UVPD lipid characterization into classical LCMS workflows to reduce the complexity of product ion spectra by resolving acyl chain length isomers chromatographically. A commercially available Escherichia coli (E. coli) lipid extract, containing a high abundance of monounsaturated PE lipid, was subjected to ${ }^{13} \mathrm{C}$ TrEnDi modification and analyzed via LCMS and UVPD ion activation. Two major modified lipids from the extract are characterized in Figure 7.2.5 to show the applicability of UVPD ion activation on a LC timescale. Parent ion peaks of $\mathrm{m} / \mathrm{z} 750.5703$ and 778.6174 were determined to be $\left[\mathrm{PE}(32: 1)+\left({ }^{13} \mathrm{CH}_{3}\right)_{4}\right]^{+}$and $\left[\mathrm{PE}(34: 1)+\left({ }^{13} \mathrm{CH}_{3}\right)_{4}\right]^{+}$after Lipid MAP Tools Structure Database search. In each UVPD spectrum fragment ion of m/z 202.1016 is observed and verifies that presence of ${ }^{13} \mathrm{C}$-methylated $\mathrm{PE}$ headgroup. On closer inspection of each product ion spectrum, a pair of fragment ions consistent with losses pertaining to fatty acyl unsaturations are observed. The insets of Figure 7.2.5 both reveal 
losses indicative of $\omega^{7}$ unsaturation. Fatty acyl composition of unmodified lipids was not obtained thus we can identify the GPLs as $\operatorname{PE}\left(32: 1, \omega^{7}\right)$ and $\operatorname{PE}\left(34: 1, \omega^{7}\right)$. Further investigation into the low abundace fragment ions as well as optimization of instrument parameters is ongoing to facilitate the identification of GPLs structures in E. coli extract via LCMS.
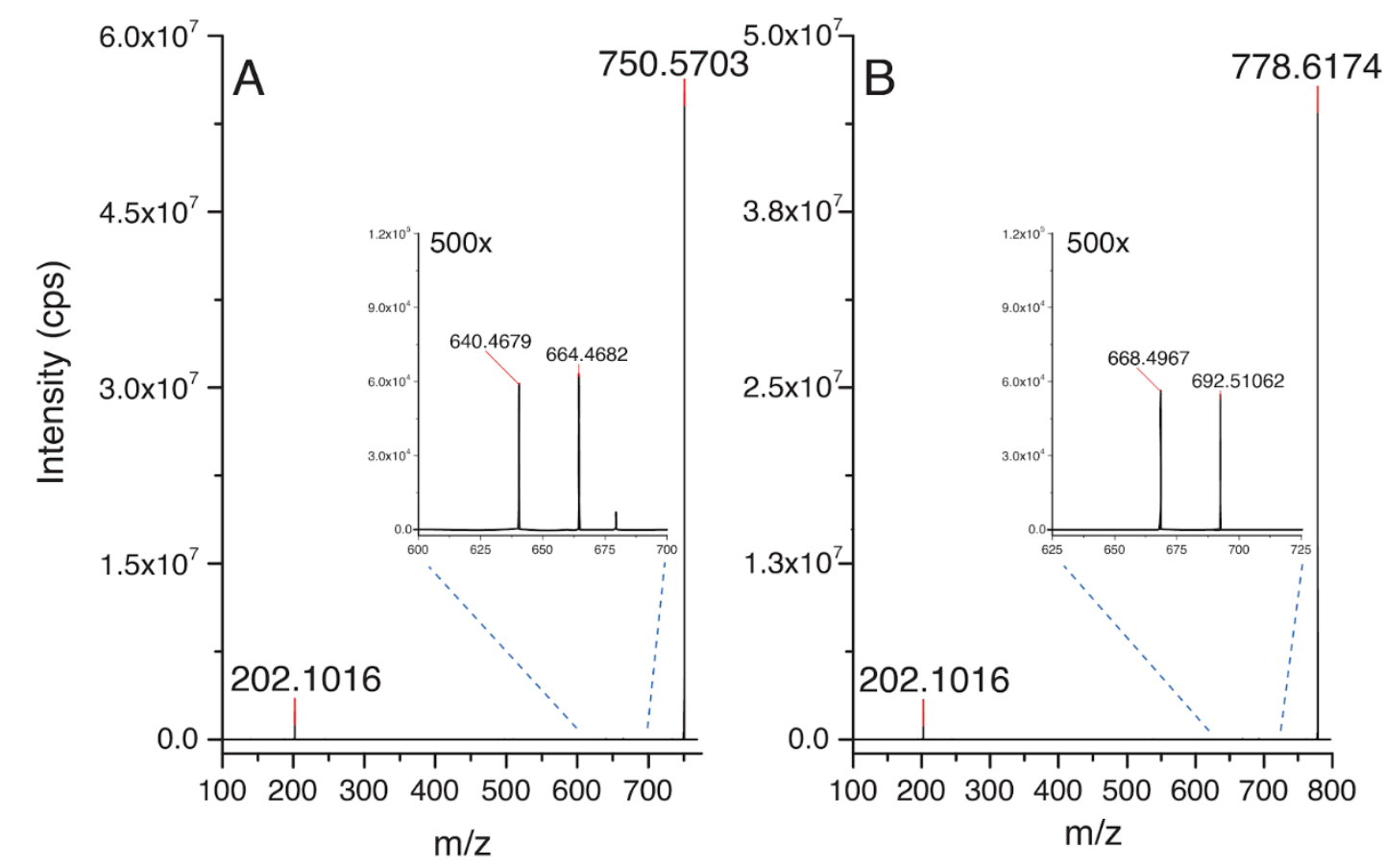

Figure 7.2.5 UVPD fragmentation of $\mathrm{m} / \mathrm{z} 750.57$ (A) and 778.62 (B) from ${ }^{13} \mathrm{C}$ TrEnDi modified polar E.coli extract analyzed by LCMS

\subsection{Ethylation of PE(16:0/18:1) using Diazoethane produced in situ from ethylated nitroso urea}

In Chapter Four the ethylation of PC and SM species with a novel apparatus for subjecting analytes to gaseous diazoethane (DZE) was performed. Phosphatidylethanolamine (PE) are another class of lipids that readily react with 
diazoalkanes due to an ethanolamine moiety at $s n 3$ position. By using the same conditions previously mentioned, a $\mathrm{PE}(16: 0 / 18: 1)$ standard was submitted to react with diazoethane generated via the alkaline decomposition of $N$-ethyl- $N$-nitroso urea. Generally, the reaction of PE lipids with DZM yielded a fully alkylated fixed net-positively charged ammonium species $\left([\mathrm{PE}+4 \mathrm{Me}]^{+}\right)$with less than $1 \%$ other lower methylation states. We opted to experiment with DZE as an alternative to DZM to explore the chemical properties of ethylated PLs via LCMS.

Each of the four possible alkylation products highlighted in Figure 7.3.1 were detected in the reaction mixture of DZE and $\mathrm{PE}(16: 0 / 18: 1)$. The mixture was analyzed via LCMS (experimental conditions in Chapter 4) and resulted in a product distribution on average $0.1 \%(0 \mathrm{Et}), 8.9 \%(1 \mathrm{Et}), 10.4 \%(2 \mathrm{Et}), 38.7 \%(3 \mathrm{Et})$ and $41.9 \%(4 \mathrm{Et})$. The increased steric bulk of the ethyl versus methyl electrophile is hypothesized to contribute to the slower reaction conversion of lower ethylation states to PE+4Et. Chromatographic traces of each are shown in Figure 7.3.2(B-D). The first extracted ion chromatogram (Figure 7.3.2A) represents unmodified $[\mathrm{PE}+\mathrm{H}]^{+}$prior to the reaction and was observed at a retention time of 10.39 minutes. Furthermore, $[\mathrm{PE}+\mathrm{Et}+\mathrm{H}]^{+},[\mathrm{PE}+2 \mathrm{Et}+\mathrm{H}]^{+}$and $[\mathrm{PE}+3 \mathrm{Et}+\mathrm{H}]^{+}$follow the expected trend of increasing retention on $\mathrm{C} 18$ stationary phase with increasing hydrophobicity and $[\mathrm{PE}+4 \mathrm{Et}]^{+}$shows a decreased retention compared to unmodified due to the ionic nature of the tetra alkyl ammonium moiety. A small amount ( $\sim \%$ ) of an ethylation positional isomer was also observed in the chromatogram resulting from $\mathrm{m} / \mathrm{z} 802.63$ at 10.39 minutes (see Chapter 5). Efforts to optimize reaction conditions are ongoing. 


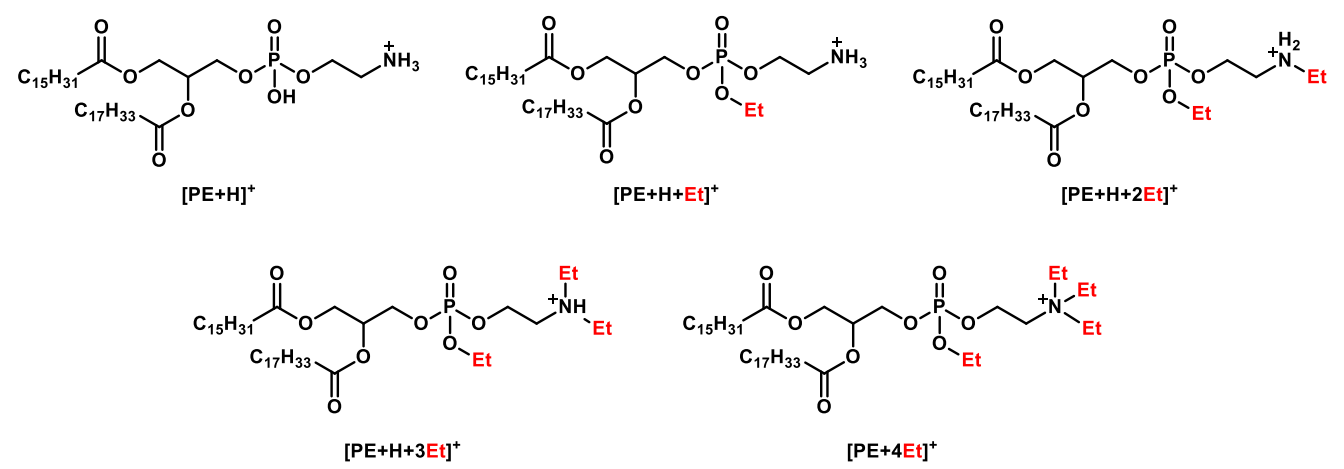

Figure 7.3.1 Structures of PE (16:0/18:1) and ethylated products produced via the reaction with diazoethane
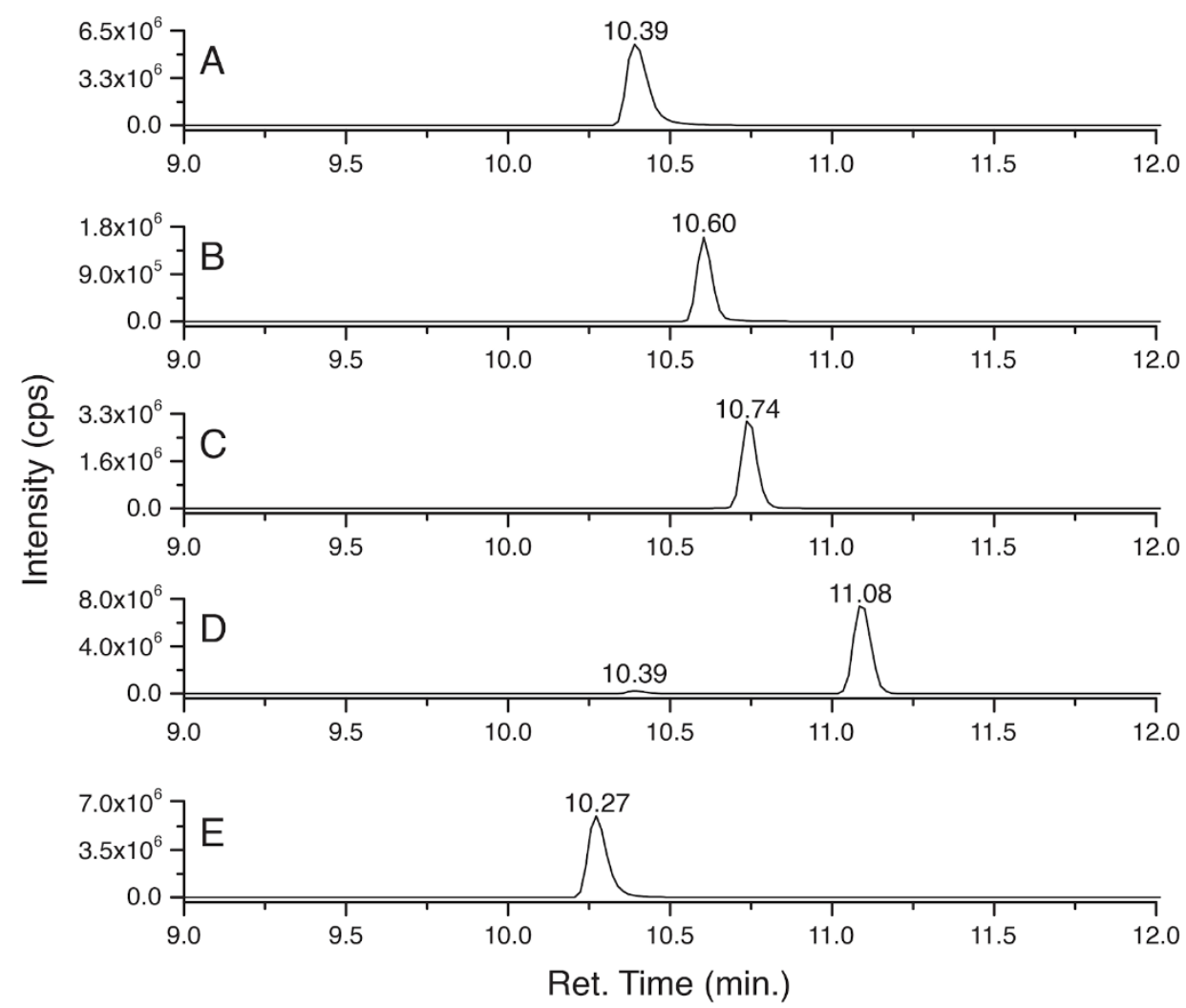

Figure 7.3.2 LCMS analysis showing of PE (16:0/18:1) prior to reaction with diazoethane (A) and mono-, di-, tri-, and tetra ethylated products produced from the reaction (B-E), respectfully 
Along with HRMS identification of each ethylated PE species, we used tandem MS to characterize each product. Figure 7.3.3A shows the CID product ion spectrum of $\mathrm{m} / \mathrm{z}$ 746.56. The most abundant fragment ion is the neutral loss of headgroup $\mathrm{EtHPO}_{4} \mathrm{CH}_{2} \mathrm{CH}_{2} \mathrm{NH}_{2}$ at $\mathrm{m} / \mathrm{z} 577.51$ and less abundant fragments at $\mathrm{m} / \mathrm{z} 239.23$ and 265.25 indicative of acylium ions of fatty acyl components. After the second ethylation of PE MS² spectrum shows similar fragments with the addition of headgroup $\left[\mathrm{EtHPO}_{4} \mathrm{CH}_{2} \mathrm{CH}_{2} \mathrm{NHEt}+\mathrm{H}\right]^{+}$at $\mathrm{m} / \mathrm{z}$ 198.09. Proposed fragment structures are shown in Figure 7.3.4. The results of triply ethylated PE fragmentation again shows $\left[\mathrm{EtHPO}_{4} \mathrm{CH}_{2} \mathrm{CH}_{2} \mathrm{NEt}_{2}+\mathrm{H}\right]^{+}$at $\mathrm{m} / \mathrm{z}$ 226.12. However in the product ion spectrum of $[\mathrm{PE}+3 \mathrm{Et}+\mathrm{H}]^{+}$a prominent $N, N$-diethylaziridinium ion (Figure $7.3 .3 \mathrm{C}$ ) is observed at $\mathrm{m} / \mathrm{z}$ 100.11. The presence of this fragment lends strong evidence that PE $+3 E t$ is ethylated once at the phosphate $\mathrm{OH}$ and twice at the ethanolamine $\mathrm{NH}_{2}$. Finally, product ion spectrum of $\mathrm{PE}+4 \mathrm{Et}$ at $\mathrm{m} / \mathrm{z} 254.15$ shows only completely alkylated headgroup ion as the major fragmentation pathway, similar to that of fully methylated PE (Chapter 4). Due to the increasing gas-phase proton affinity of the alkylated amine, product ions produced by fragmentation of the head group of the respective ethylated PE products become more abundant. In conclusion ethylation of PE to a fully alkylated ammonium species is challenging due to steric bulk of ethyl vs methyl. Each ethylated product was observed by LCMS and characterized by $\mathrm{MS}^{2}$. 

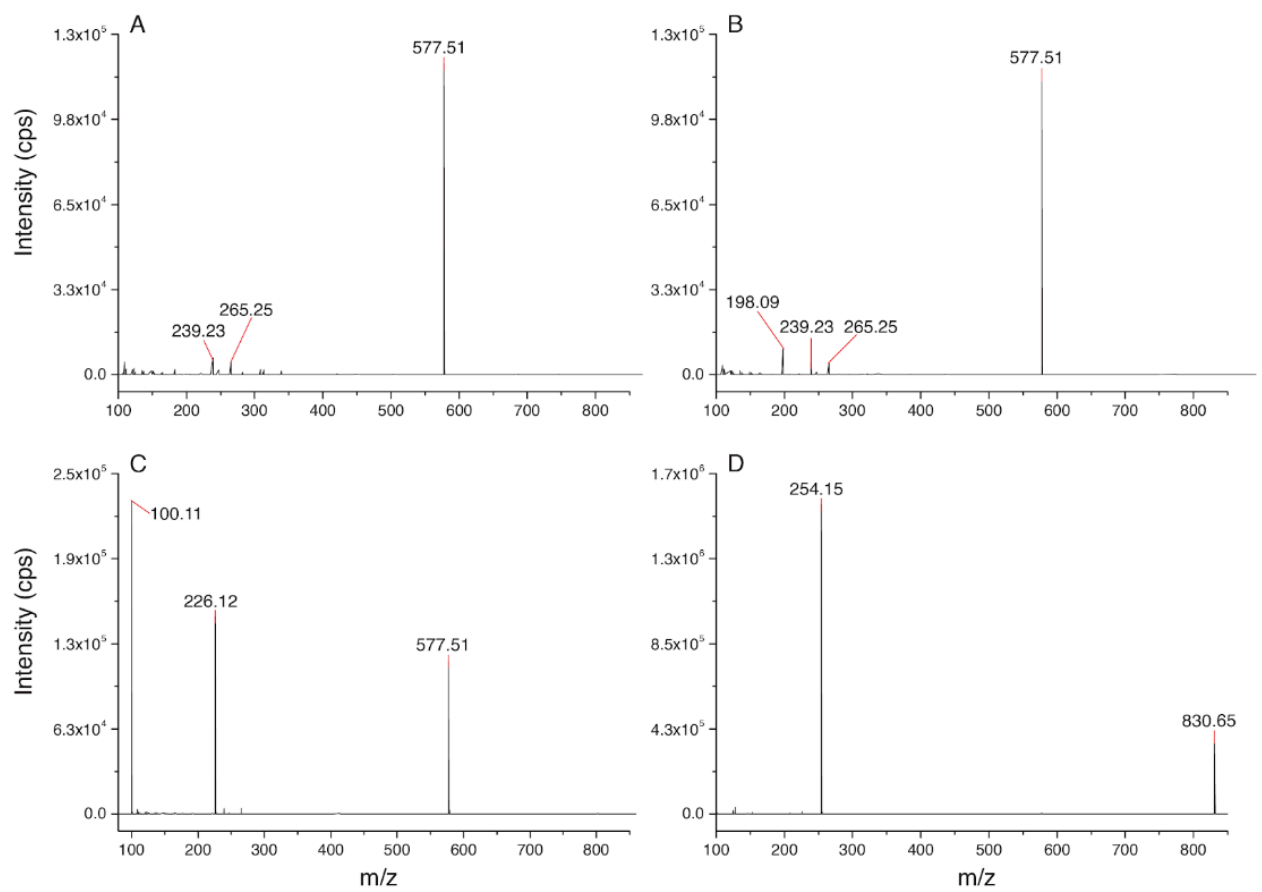

Figure 7.3.3 Tandem MS analysis of mono- (A), di- (B), tri-(C) and tetra-ethylated (D) PE products produced in the reaction with diazoethane

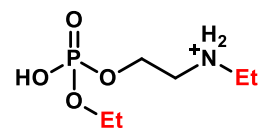

$\mathrm{m} / \mathrm{z} 198.09$

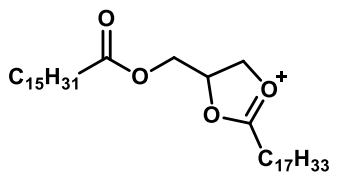

$\mathrm{m} / \mathrm{z} \mathbf{5 7 7 . 5 2}$<smiles>CCOP(=O)(O)OCC[NH+](CC)CC</smiles>

$\mathrm{m} / \mathrm{z} 226.12$

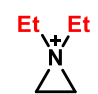

$\mathrm{m} / \mathrm{z} 100.11$<smiles>CCOP(=O)(O)OCC[N+](CC)(CC)CC</smiles>

Figure 7.3.4 Identified fragments from the analysis of ethylated PE products via $\mathbf{M S}^{2}$ 


\subsection{Conclusion}

The results presented in this chapter show the application of TrEnDi modification benefits the deeper structural characterization of GPLs. Modification of PC and PE lipids in combination with charge inversion ion/ion reactions and UVPD fragmentation provides a much-needed signal increase to confidently characterize fatty acyl components and carbon-carbon double bond location. These works clearly demonstrate the benefits of TrEnDi to continue to expand and evolve the MS analysis of GPLs. The unpublished results reported in this herein constitute the foundation for several ongoing lines of research and will be published in due course.

\subsection{References}

(1) Pi, J.; Wu, X.; Feng, Y. Anal. Methods 2016, 8 (6), 1319-1332.

(2) Stutzman, J. R.; Blanksby, S. J.; McLuckey, S. A. Anal. Chem. 2013, 85 (7), $3752-3757$.

(3) Rojas-Betancourt, S.; Stutzman, J. R.; Londry, F. A.; Blanksby, S. J.; McLuckey, S. A. Anal. Chem. 2015, 87 (22), 11255-11262.

(4) D'Auria, M. Photochem. Photobiol. Sci. 2019, 18 (10), 2297-2362.

(5) Murphy, R. C.; Okuno, T.; Johnson, C. A.; Barkley, R. M. Anal. Chem. 2017, 89 (16), 8545-8553.

(6) Franklin, E. T.; Betancourt, S. K.; Randolph, C. E.; McLuckey, S. A.; Xia, Y. Anal. Bioanal. Chem. 2019, 411 (19), 4739-4749.

(7) Steckel, A.; Schlosser, G. Molecules 2019, 24 (3), 1-11.

(8) Hart-Smith, G. Anal. Chim. Acta 2014, 808, 44-55. 
(9) Brodbelt, J. S. Chem. Soc. Rev. 2014, 43 (8), 2757-2783.

(10) Stedwell, C.; Polfer, N. Lecture Notes In Chemistry 83 Laser Photodissociation and Spectroscopy of Mass-Separated Biomolecular Ions; Polfer, N. C., Dugourd, P., Eds.; Springer International Publishing: New york, 2010.

(11) Pérot, M.; Lucas, B.; Barat, M.; Fayeton, J. A.; Jouvet, C. J. Phys. Chem. A 2010, $114(9), 3147-3156$.

(12) Reilly, J. Mass Spectrom. Rev. 2009, 28 (February 2008), 425-447.

(13) March, R. E. J. Mass Spectrom. 1997, 32 (4), 351-369.

(14) Vasicek, L. A.; Ledvina, A. R.; Shaw, J.; Griep-Raming, J.; Westphall, M. S.; Coon, J. J.; Brodbelt, J. S. J. Am. Soc. Mass Spectrom. 2011, 22 (6), 1105-1108.

(15) Thermo Scientific. Orbitrap Fusion Lumos https://planetorbitrap.com/uvpd\#tab:technology (accessed Nov 21, 2019).

(16) Reinink, P.; Buter, J.; Mishra, V. K.; Ishikawa, E.; Cheng, T. Y.; Willemsen, P. T. J.; Porwollik, S.; Brennan, P. J.; Heinz, E.; Mayfield, J. A.; et al. J. Exp. Med. 2019, $216(4), 757-771$.

(17) Mishra, V. K.; Buter, J.; Blevins, M. S.; Witte, M. D.; Van Rhijn, I.; Moody, D. B.; Brodbelt, J. S.; Minnaard, A. J. Org. Lett. 2019, 21 (13), 5126-5131.

(18) Marrakchi, H.; Lanéelle, M. A.; Daffé, M. Chem. Biol. 2014, 21 (1), 67-85.

(19) Macias, L. A.; Feider, C. L.; Eberlin, L. S.; Brodbelt, J. S. Anal. Chem. 2019, 91 (19), 12509-12516. 


\section{Chapter 8: Conclusion}

In this thesis a series of experimental studies have been presented which I believe have made important contributions to the field of chemically modifying of biomolecules and xenobiotics. Following our initial methodology for using diazomethane to methylate phospholipids (Chapter 2), we have studied in detail the reactivity of plasmalogen lipids with ${ }^{13} \mathrm{C}$-TrEnDi (Chapter 3), demonstrated the use of a safe alternative to diazoalkane generation (Chapter 4), and exhibited the benefit of iTrEnDi modification to the pesticide

glyphosate (Chapter 5). We have also shown the capability of TrEnDi modified lipids to enhance other analytical strategies such as the ion/ion reactions to determine fatty acyl chain composition, the reaction with acetone to form oxetanes susceptible to CID fragmentation, and UVPD activation on the LC time scale to locate unsaturations in fatty acids in a complex mixture (Chapter 7). These advances summarized in this work is but a small advancement in a field that is growing at a rapid pace but will have a lasting impact on future generations of analytical chemists in our lab. 


\section{Appendices}

Appendix A 


\section{Bibliography or References}

Each chapter has references included at the end 